\title{
INVESTIGATION OF GENES INVOLVED IN SOMATIC \\ EMBRYOGENESIS AND PLANT-AGROBACTERIUM INTERACTIONS THROUGH TRANSCRIPTIONAL PROFILING
}

\begin{tabular}{c} 
A Dissertation \\
presented to \\
the Faculty of the Graduate School \\
at the University of Missouri-Columbia \\
In Partial Fulfillment \\
of the Requirements for the Degree \\
Doctor of Philosophy \\
\hline Dr. Bing Yang, Dissertation Supervisor \\
MAY 2021
\end{tabular}


The undersigned, appointed by the dean of the Graduate School, have examined the dissertation entitled

\section{INVESTIGATION OF GENES INVOLVED IN SOMATIC EMBRYOGENESIS AND PLANT-AGROBACTERIUM INTERACTIONS THROUGH TRANSCRIPTIONAL PROFILING}

presented by Christopher Willig,

a candidate for the degree of Doctor of Philosophy

and hereby certify that, in their opinion, it is worthy of acceptance.

Dr. Bing Yang

Dr. Walter Gassmann

Dr. Felix Fritschi

Dr. Emmanuel Liscum 


\section{DEDICATION}

This work is dedicated to Honey

for always being by my side

We miss you

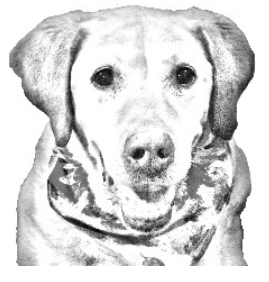




\section{ACKNOWLEDGEMENTS}

First and foremost, I want to thank my wife Kyley for her patience and support as I wrote this dissertation and just in general throughout my graduate education. I want to thank Dr. Zhanyuan Zhang for taking me on as a graduate student and for the training, mentoring, advice, and feedback he provided me as my academic supervisor. I also want to thank my dissertation committee members for their crucial support: Dr. Bing Yang who was willing to step in as committee chair and de facto advisor during my final year of graduate school, Dr. Walter Gassmann, for his advice and encouragement, Dr. Felix Fritschi, for helping to set me up with the plot space and resources to grow stock plants in the field, and Dr. Mannie Liscum for providing me with a laboratory rotation experience during my first year and for helping me to navigate difficult situations on multiple occasions (and taking me to see Megadeth in Chicago that one time). I also want to acknowledge my former committee members who have gone into retirement, Dr. Jeanne Mihail for her valuable advice and for instilling me with a solid working knowledge of plant anatomy, and Dr. Bill Spollen for sacrificing many hours of his time to work with me one-on-one teaching me how to analyze RNA-seq data when I understood next to nothing about bioinformatics.

Thank you to Drs. Alexander Jurkevich and Frank Baker of the Molecular Cytology Core Facility for their provision of training and advice on microscopy, to Nathan Bivens and Kate Shipova of the DNA core facility for consulting on difficult sequencing projects and helping me getting ahold of everything I ever needed for cloning as quickly 
as possible. I also want to thank to MU Research Computing Support Services team, especially Asif Ahamed and Ashkan Mirzaee for helping get me set up on the Lewis cluster and helping troubleshoot software problems on several occasions.

I want to thank past members of Dr. Zhang's lab and the MU Plant Transformation Core Facility, some of whom contributed to the work in this dissertation, especially Phat Tien Do (thanks for teaching me sorghum and maize transformation and being patient with me while I learned), Kaixuan Duan, Muruganantham Mookkan, Michelle (Folta) Valentine, Hyeyoung Lee, and Neng Wan. Thank you to Matt Craven for the laboratory assistance you provided as my undergraduate mentee, and thanks for your dependability even as I was spending most of my time working on writing.

Other groups I want to thank include Nick Kalaitzandonakes and Ken Zahringer of the Economics and Management of Agrobiotechnology Center for support and encouragement and for providing me with a stimulating side-project and speaking opportunities, Loriana Sekarski and the whole PTLS team who helped me develop soft skills, which I used to better manage my time and resources, and Debbie Allen, Dr. Mark Hannink, and the MU Life Sciences Fellowship for helping develop my presentation skills and providing support during my first four years of graduate study.

Finally, I want to thank my parents, brothers, and other family members and friends for emotional support and their understanding as I wasn't available as much as I should have been. Thanks mom, dad, Blake, Gage, Ryan, Adam, Desiree, Steven, Dave, Alicia, and Alli. 


\section{TABLE OF CONTENTS}

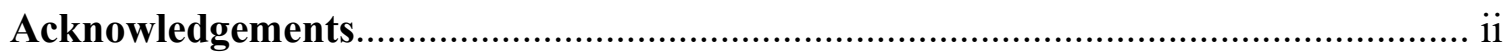

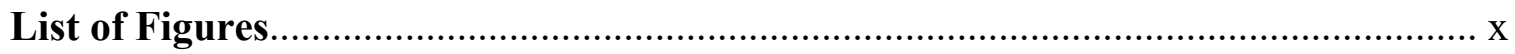

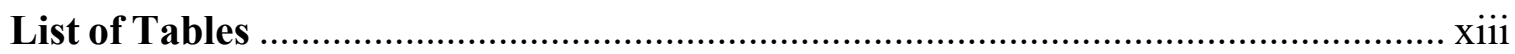

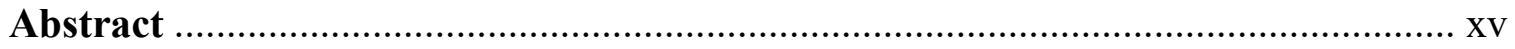

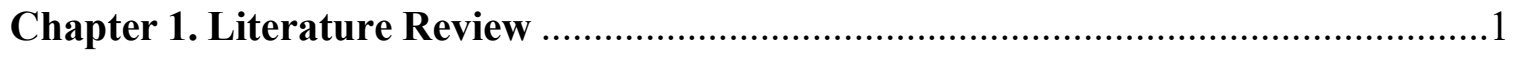

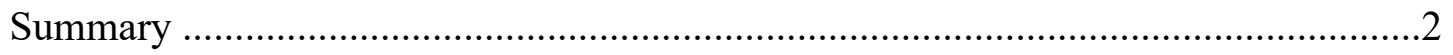

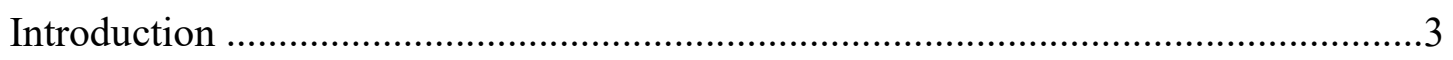

Impact of plant biotechnology on agriculture ............................................

History and applications of plant tissue culture ...............................................4

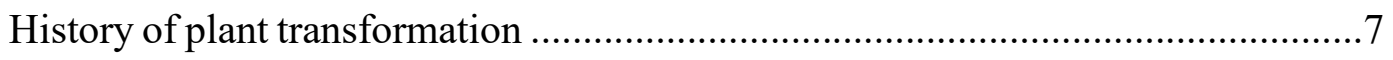

Emerging biotechnological methods .................................................... 10

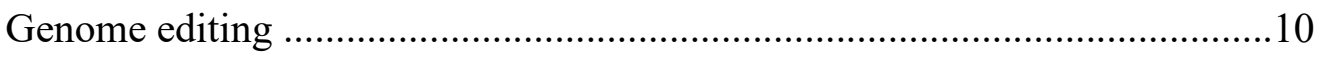

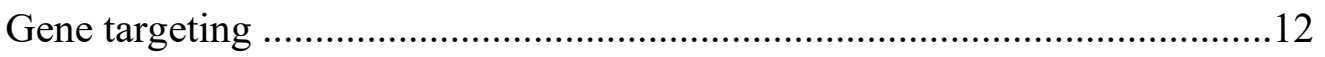

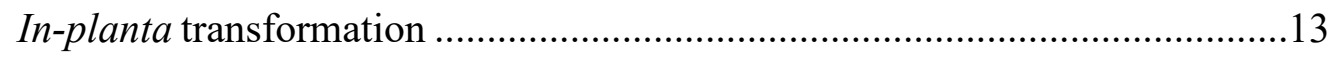

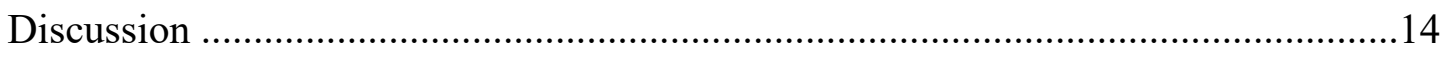

A detailed look at the somatic embryogenesis process ....................................14

Overview of morphological and developmental characteristics of somatic em-

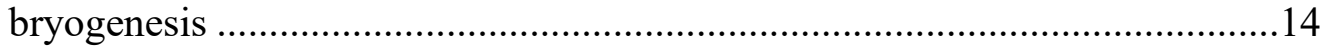

Genetic basis of somatic embryogenesis ............................................... 18

A detailed look at Agrobacterium-plant interactions surrounding transformation 
Overview of Agrobacterium biology, pathogenesis, and DNA transfer mechanism .25

Using differential gene expression to examine host genes interacting with $\mathrm{Ag}$ robacterium infection .29

Transcriptomic analysis in model plant systems ..................................... 31

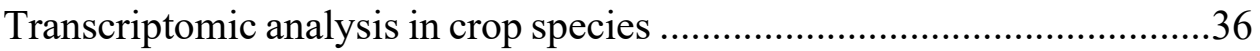

Transcriptional response to heterologous expression of vir genes in host plants

Conclusion .43

Advantages and limitations of transcriptomic analysis as an approach to discover plant genes and pathways associated with somatic embryogenesis and Agrobacterium-mediated transformation 43

Future outlook for enhancing plant regeneration and transformation .45

Reference List

\section{Chapter 2. Using transcriptional dynamics to uncover genes expressed during so-} matic embryogenesis in Sorghum bicolor

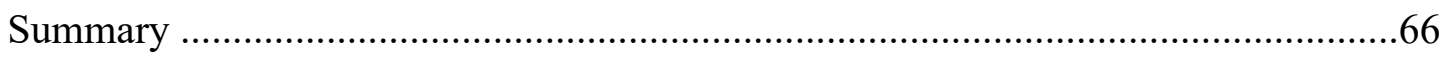

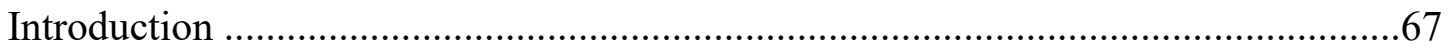

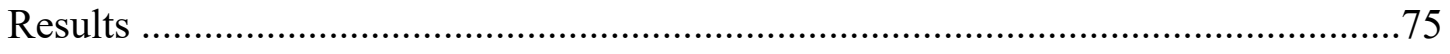

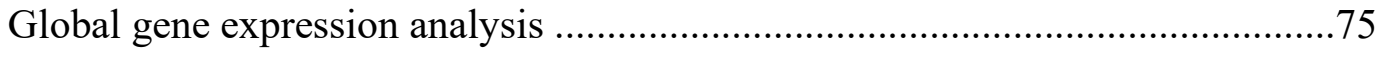

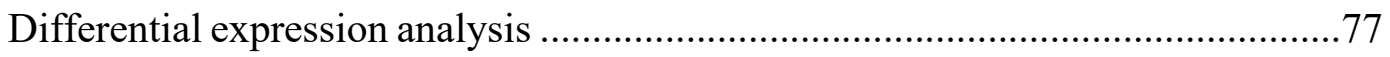

Comparisons made through time within conditions ….................................... 78 
Iso-temporal comparisons made between conditions ..........................................8

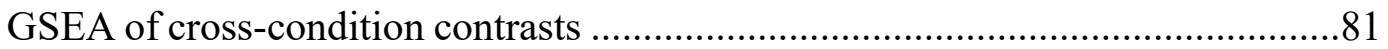

Gene mining based on important gene functional classes .....................................85

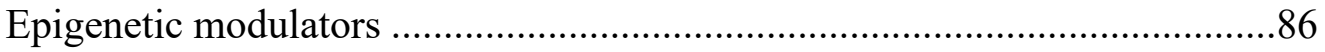

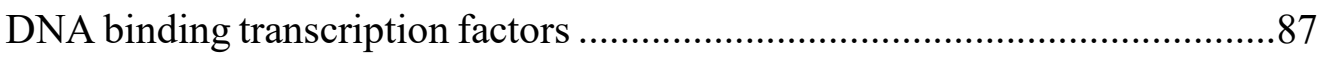

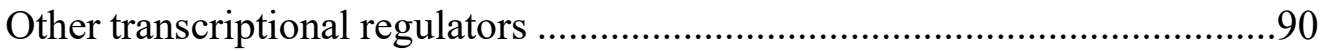

Hormone-related genes ..............................................................................

Other functional categories ........................................................................99

Comparison of differentially expressed genes to results from other studies ...........96

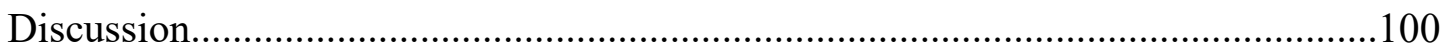

Assessment of differentially expressed genes by functional category ..................100

Comparison to findings of similar studies ........................................................106

Potential improvements to study design .......................................................107

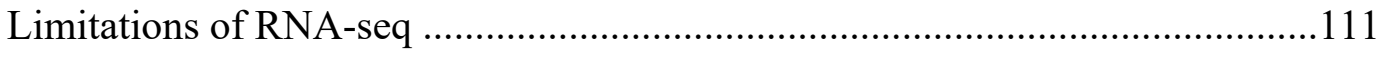

Conclusion and future perspectives ..................................................................113

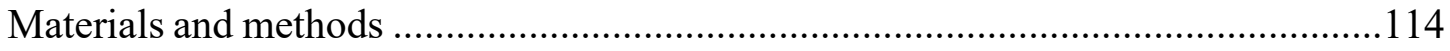

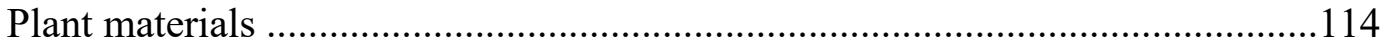

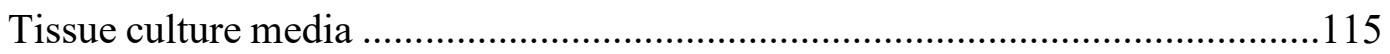

Embryo tissue culture initiation and collection ................................................116

Sample processing and transcriptome sequencing ..........................................116

Data processing and gene expression quantification ..........................................117

Differential expression analysis ..................................................................118

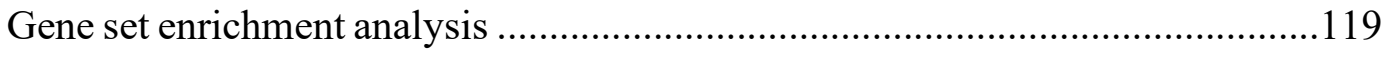

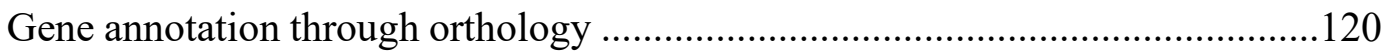


Reference List

Chapter 3. Investigation of host response in the Agrobacterium-plant interaction using

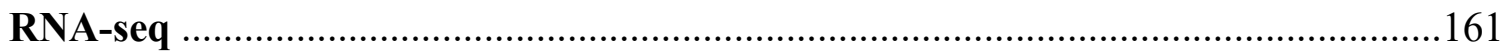

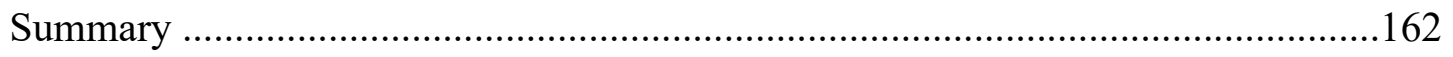

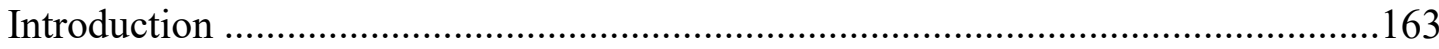

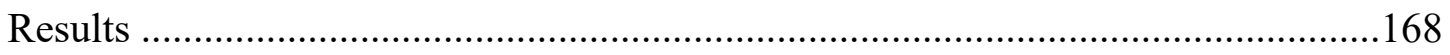

RNA-seq sample collection and read alignment ..............................................168

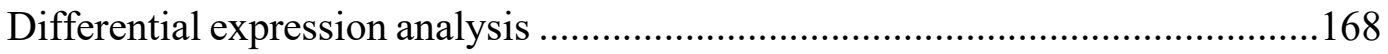

Highly differentially expressed genes .............................................................172

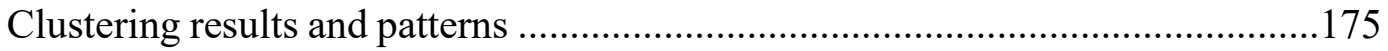

Gene set enrichment analysis ....................................................................176

Differentially expressed transcription factor genes .............................................180

Differentially expressed hormone-related genes ..............................................182

Genes responding to virulent Agrobacterium treatment ...................................185

Comparison of RNA-seq results to qRT-PCR data ............................................187

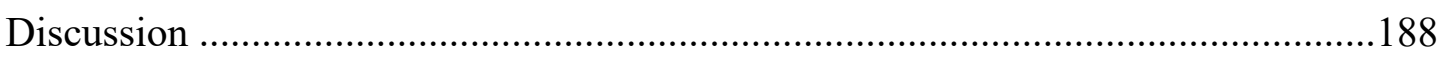

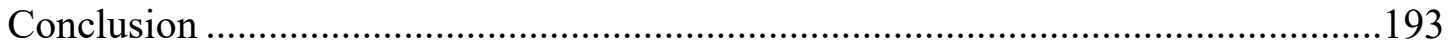

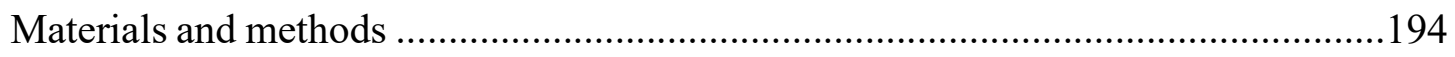

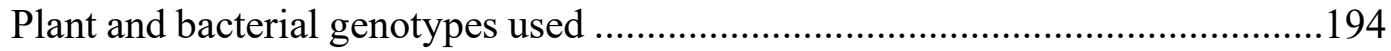

Transient seedling transformation and sample collection .....................................195

Data processing and gene expression quantification ...........................................196

Analysis of differential gene expression ...........................................................197 
Scatterplots, clustering, and gene set enrichment analysis

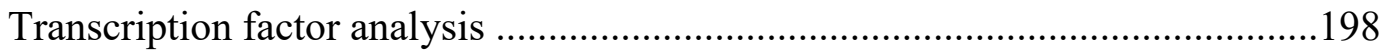

Evaluating expression trends with qRT-PCR ...............................................199

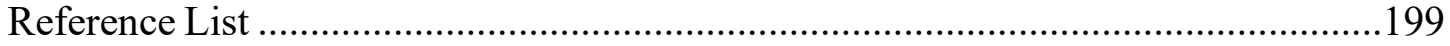

Chapter 4. Evaluation of the influence of candidate genes on somatic embryogenesis

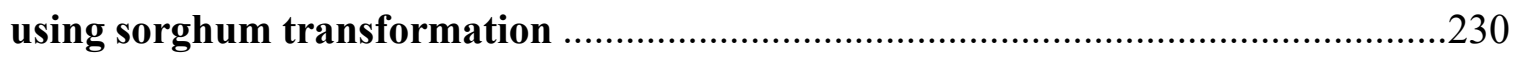

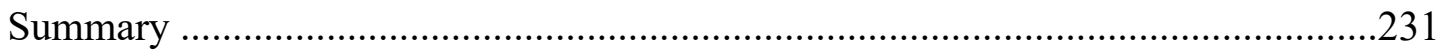

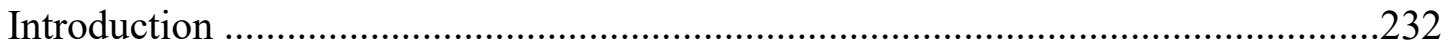

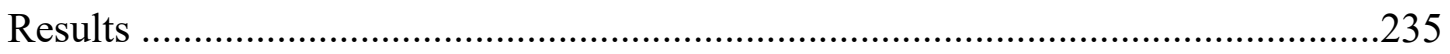

Functional testing of candidate genes through sorghum transformation .............235

Silencing of candidate genes using RNAi ...................................................236

Constitutive overexpression of maize orthologs of candidate genes in a non-regen-

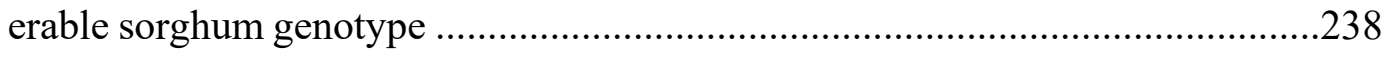

Inducible expression of candidate genes in sorghum lines ..............................240

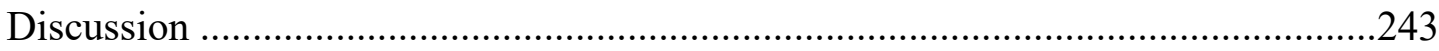

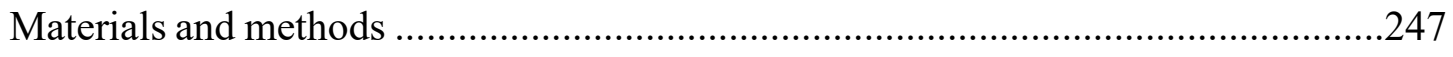

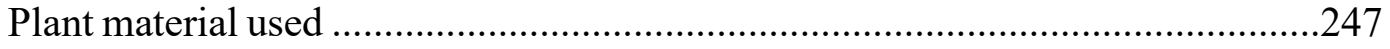

Candidate gene cloning and plasmid construction .....................................249

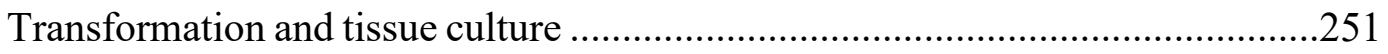

Assessment of marker gene expression in inducible system ............................253

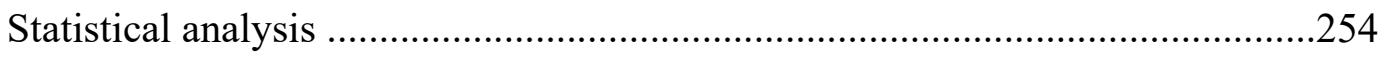

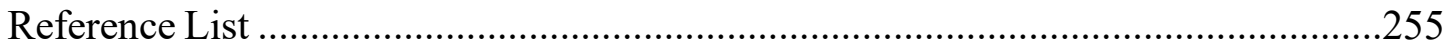


Vita

.288 


\section{LIST OF FIGURES}

$\begin{array}{ll}\text { Figures } & \text { Pages }\end{array}$

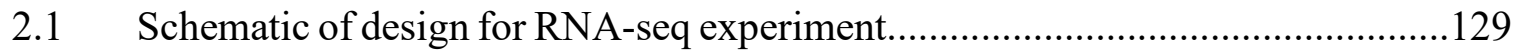

2.2 MDS plots showing relationships between samples......................................130

2.3 Sets of genes differentially expressed over time within the same experimental condition.

2.4 Selected overrepresented gene sets among upregulated and downregulated genes at each cross-condition contrast.

2.5 Expression of all epigenetic modulator genes upregulated across conditions.....134

2.6 Expression of selected WRKY family transcription factor genes downregulated

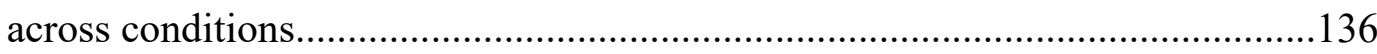

2.7 Expression of selected AP2/ERF family transcription factor genes upregulated across conditions.

2.8 Expression of selected bHLH and NAC domain-containing transcription factor genes upregulated across conditions.

2.9 Expression of selected transcription factor genes from various families upregulated across conditions. .141

2.10 Expression curves of selected differentially expressed auxin-related genes........143

2.11 Expression curves of selected differentially expressed hormone (other than auxin)related genes 145

2.12 Expression curves of selected homeodomain-like superfamily genes. 147

3.1 PCA plot showing sample distribution. .205 
3.2 Scatterplots displaying relative expression of all genes in each treatment at each

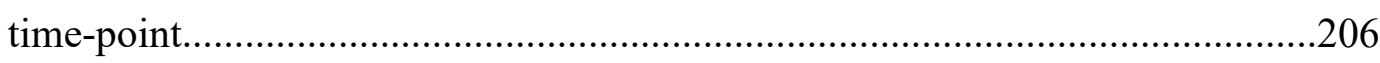

3.3 Venn diagrams showing the differentially expressed gene sets.............................208

3.4 Co-expression clustering of differentially expressed genes................................210

3.5 Selected overrepresented gene sets among upregulated and downregulated genes at

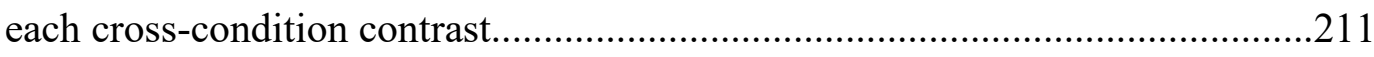

3.6 Pie charts showing differentially expressed transcription factor genes................212

3.7 Expression of select transcription factor genes displaying clear upregulation in both Agrobacterium treatments relative to the mock....................................................214

3.8 Expression of select transcription factor genes displaying clear downregulation in both Agrobacterium treatments relative to the mock............................................216

3.9 Select differentially expressed genes related to the hormones auxin, ethylene, gib-

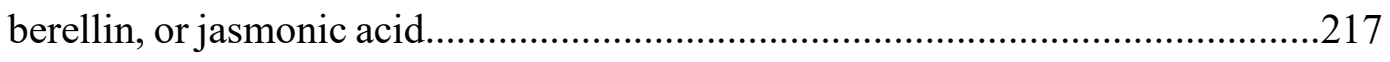

3.10 Select differentially expressed genes related to cytokinin..................................219

3.11 Genes differentially expressed only in the virulent $v s$. mock comparison showing apparent differences in expression trends between the virulent and avirulent treat-

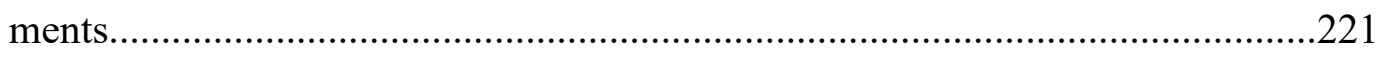

3.12 Relative expression for select genes quantified by qRT-PCR ...............................223

4.1 The process of somatic embryogenesis in sorghum..........................................259

4.2 Overall effect of particle bombardment and Agrobacterium-mediated transfor-

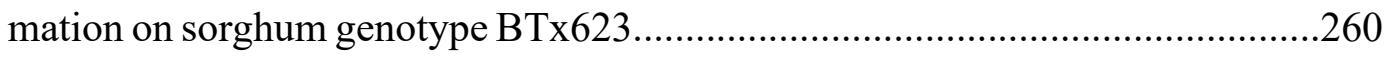


4.3 Representative $N$. benthamiana leaf disk samples for testing of marker gene expression in the bi-directional dexamethasone-inducible construct (pFGC-DEXeCFPGUS)

4.4 Test of DEX-inducible CFP expression in maize B73 immature embryos transformed with the construct containing the pOp6 promoter......................................264

4.5 Representative and composite plasmids used in this study displaying essential se-

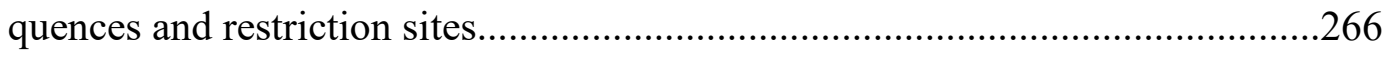

4.6 RNAi sequences ordered for DNA synthesis targeting 10 candidate genes.........271 


\section{LIST OF TABLES}

Tables

Pages

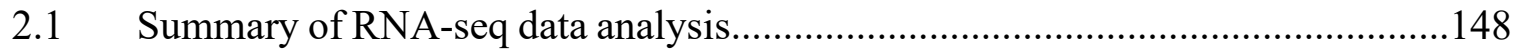

2.2 Most highly upregulated genes in contrasts made across time...........................150

2.3 Most highly downregulated genes in contrasts made across time......................153

2.4 Summary of differentially expressed genes in cross-condition comparisons.......156

2.5 Most highly upregulated genes in contrasts made across conditions..................157

2.6 Most highly downregulated genes in contrasts made across conditions..............159

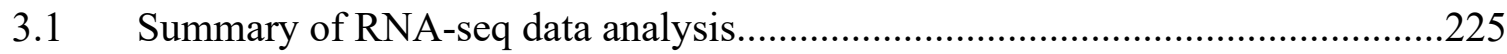

3.2 Highly upregulated genes in avirulent $v s$. mock and virulent $v s$. mock comparisons

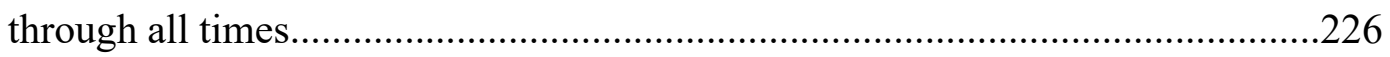

3.3 Highly downregulated genes in avirulent vs. mock and virulent vs. mock compari-

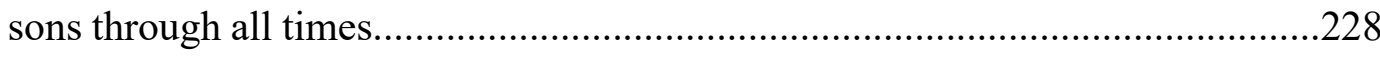

4.1 Results of Agrobacterium-mediated transformation RNAi experiment 1 in sorghum P898012. .275

4.2 Maize full-length cDNA clones obtained that are orthologous to candidate sorghum genes. .276

4.3 Results of Agrobacterium-mediated transformation RNAi experiment 2 in sorghum P898012. .277

4.4 Results of Agrobacterium-mediated transformation RNAi experiment 3 in sorghum P898012. 277

4.5 Results of particle bombardment RNAi experiment in sorghum Tx430...........277 
4.6 Results of Agrobacterium-mediated transformation overexpression experiment in

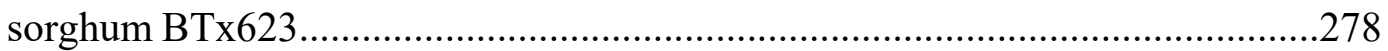

4.7 Results of particle bombardment overexpression experiment in sorghum ВТх623

4.8 Results of Agrobacterium-mediated transformation DEX-inducible expression ex-

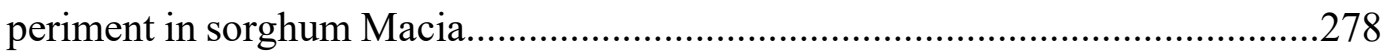

4.9 Results of Results of Agrobacterium-mediated transformation DEX-inducible ex-

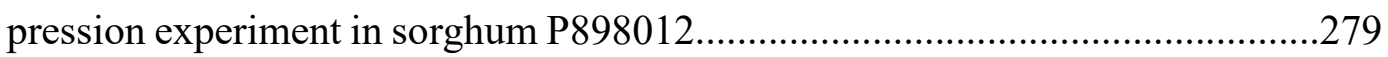




\title{
INVESTIGATION OF GENES INVOLVED IN SOMATIC EMBRYOGENESIS AND PLANT- $A G R O B A C T E R I U M$ INTERACTIONS THROUGH TRANSCRIPTIONAL PROFILING
}

\author{
Christopher Willig
}

Dr. Bing Yang, Dissertation Supervisor

\begin{abstract}
Plant genetic engineering relies on the ability to transmit and express cloned DNA sequences in plant cells (transformation) as well as the capacity for the cells carrying this DNA to undergo division and differentiation (regeneration), eventually giving rise to a mature whole plant. The breadth of application for genetic engineering is limited by constraints on one or both of these factors in many plant species and individual varieties. Uncovering plant genes which are involved in important aspects of either component can inform the development of technologies that serve to enable or improve the efficiency of genetic modification methods. The most commonly employed method of delivering exogenous genetic material into plant cells is via disarmed strains of the plant pathogen $\mathrm{Ag}$ robacterium tumefaciens. Somatic embryogenesis is a frequently applied mode of plant regeneration following DNA delivery, especially in major cereal crops such as maize, rice, and sorghum. In the work reported here, whole transcriptome sequencing (RNA-seq) was used in two different experiments to capture transcriptional dynamics throughout early somatic embryogenesis in immature zygotic embryo tissue of the major crop plant sorghum (Sorghum bicolor), and throughout early times following host plant inoculation with A. tumefaciens in seedlings of the model plant Arabidopsis thaliana (Chapters 2 and 3 , respectively). In both cases, differential expression analysis revealed many genes which were induced either during somatic embryogenesis or in response to inoculation
\end{abstract}


with either virulent or avirulent $A$. tumefaciens strains. Several of these genes were highlighted as candidates for future study into their potential role in the respective processes. Multiple candidate genes were functionally tested, using transgenic methods, for the possibility of having a role in the regulation of somatic embryogenesis in sorghum (Chapter 4). These experiments failed to confirm an influence over the process for all candidate genes that were evaluated. The experimental work documented herein contributes to a growing body of literature documenting plant genes which could serve as possible targets for techniques that work to enhance the utility of biotechnological methods for improving traits in plants. 


\section{Chapter 1}

\section{Literature Review}

Note: Some of the information in this chapter was published under the title:

Willig C.J., Duan K., Zhang Z.J. (2018) Transcriptome profiling of plant genes in response to Agrobacterium tumefaciens-mediated transformation. In Agrobacterium Biology, ed. S.B. Gelvin p319-348. https://doi.org/10.1007/82 2018115 


\section{Summary}

Plant genetic modification is enabled by both the ability to control the regeneration of whole plants using tissue culture, and the development of means to transmit exogenous DNA sequences into plant genomes so that they can be stably inherited, a process referred to as transformation. There are multiple routes to achieving both regeneration (e.g., somatic embryogenesis, de novo shoot organogenesis) and transformation (e.g., particle bombardment, Agrobacterium-mediated). This review will discuss what is currently understood about the genetics and key molecular interactions undergirding the processes of somatic embryogenesis and the interplay between pathogen and host associated with Agrobacterium-mediated transformation. A brief history on the origins and advancement of plant biotechnological methods is offered along with emerging technologies that have the potential to greatly expand the capacity for plant trait improvement, and yet are, to some extent, reliant on successful transformation and subsequent regeneration. Current models describing the sequence of events occurring on the morphological and molecular levels are discussed for each process, including studies elucidating key plant factors involved, with a particular focus on the use of transcriptional profiling as a means of identifying genes or gene sets implicated in either phenomenon. Finally, current progress on and prospects for advancing plant biotechnology through breakthrough techniques which are informed by an enhanced understanding of the underlying transformation and regeneration mechanisms are discussed.

\section{Introduction}




\section{Impact of plant biotechnology on agriculture}

Since the advent of agriculture between 10,000 and 12,000 years ago, humans have been modifying the genetic makeup and, consequently, the trait composition of plants cultivated for food and materials through an iterative process of conscious and unconscious selection. This selection process, called domestication, has resulted in the development of plant varieties (crops) which possess many traits favorable to human uses (e.g., loss of seed dispersal mechanism, increased seed nutritive content and size, determinate growth forms), yet are often genetically and morphologically distinct from their wild progenitors. Until the near the end of the $19^{\text {th }}$ century A.D., plant trait improvement techniques were mostly limited to seed saving based on the favorable phenotypic characteristics observed. The re-discovery, around 1900, of Gregor Mendel's work on trait inheritance unlocked the mechanism by which traits were encoded and allowed patterns of transmission to offspring to be predicted, thereby enabling the establishment of the field of genetics and modern plant breeding practices (Fisher 1936).

Throughout the $20^{\text {th }}$ century, a combination of factors including widespread employment of hybridization, improved capacity for chemical fertilizer production, technological innovation in agronomic practices, and an increasing understanding of how genes influence specific traits, led to a sharp rate of increase in crop productivity relative to that seen historically (Grassini et al. 2013). In the 1950s and 60s, an initiative termed the "Green Revolution" helped to institute the use of modern agricultural practices such as fertilizer application, mechanized planting and harvesting, and cultivation of high-yielding plant varieties in developing nations, resulting in an overall increase in global food 
security (Evenson and Gollin 2003). Scientific breeding programs have been and continue to be essential for both enhancing crop traits with respect to longstanding challenges in agriculture and for addressing newly arising problems such as disease resistance or climate stress. However, the immediacy of the response to the demands of modern agriculture is constrained by the time and resources required to identify and disseminate novel traits through a complex series of crosses and backcrosses. Advances in plant biotechnology have, only in the past four decades, afforded the potential to directly transmit a DNA sequence conferring single or multiple traits of interest into the germline of a given plant variety without also potentially introducing unwanted or even deleterious traits along with it (a concept termed "linkage drag"), as is a drawback of conventional breeding techniques. This capability can, in some contexts, expedite the introduction of advantageous traits for the benefit of growers and consumers (Mittler and Blumwald 2010).

History and applications of plant tissue culture

The practice of cultivating or manipulating plant tissues or vegetative cells under sterile artificial (in vitro) conditions to study their properties, which forms the basis for plant biotechnology, first came into use in the late 1920s. The theoretical basis for the idea that individual plant cells possess the potential to give rise to a complete plant (a concept called totipotency) originates with the Austrian botanist Gottlieb Haberlandt in a lecture presented in 1902 (Thorpe 2007). One of the earliest reports of seemingly indefinite proliferation of plant cells in tissue culture was conducted using tomato root 
tips as explants (White 1934). Over the next several years, cultures initiated with other explants such as shoot buds and zygotic embryos were reported (Loo 1945, Swamy 1958). Tissue culture offered its first practical application in plant breeding when it was reported that it could be used to rescue embryos resulting from hybrid crosses that produced seeds which were otherwise non-viable (Laibach 1929). It was discovered that explants could be manipulated to form an amorphous tissue type called "callus" in sterile culture and that plant tissue could differentiate to form shoots and roots under controlled conditions (White 1939a, b). Improvements to media formulations including the usage of essential macronutrients, micronutrients, and vitamins along with the plant hormones auxin and cytokinin improved the viability of cultured explants and expanded the range of possibilities for how tissue morphogenesis could be manipulated in studies conducted throughout the 1950s and 60s (Gamborg et al. 1976, Murashige and Skoog 1962, Skoog and Miller 1957). In addition to the culture of whole tissues, methods of initiating cultures of suspended single cells and inducing them to form cell colonies were developed (Muir et al. 1954, 1958).

Totipotent somatic embryo formation from callus tissue was first reported from carrot (Daucus carota) cell suspension culture in 1958 (Reinert 1958, Steward et al. 1958) and the first whole plant successfully derived all the way from a single cultured protoplast was achieved in tobacco a few years later (Vasil and Hildebrandt 1965). Plant regeneration via de novo shoot organogenesis from mature endosperm explants of Exocarpus cupressiformis was reported around the same time (Johri and Bhojwani 1965).

Tissue culture came into use for obtaining virus-free germplasm through culture of plant 
shoot or root tips and has been routinely employed for this purpose ever since (Panattoni et al. 2013, Quak 1961). Another practical application of tissue culture is the development of interspecific and intergenic hybrids that overcome barriers imposed by sexual incompatibility through either controlled pollination in tissue culture or through somatic hybridization involving the fusion of protoplasts derived from two different plant species, followed by subsequent cultural manipulations to regenerate a mature whole plant (Grosser et al. 2000, Liu Jihong et al. 2005). Culture of gametophytes has allowed production and maintenance of haploid cell lines (Xu et al. 2007). Treatment of cultured plant tissues with mitotic inhibitor compounds (e.g., colchicine) has enabled the development of polyploid plant lines from diploid tissue or doubled haploid lines from haploid tissue, thereby creating completely homozygous plants, which have proven to be a tremendously useful tool to assist in conventional breeding (Murovec and Bohanec 2011).

Over the last 60 years, somatic embryogenesis under optimal tissue culture conditions has been reported for hundreds of plant species (Fehér 2019). Somatic embryogenesis is unique from other modes of regeneration as it involves the emergence of clonal plantlets from non-germline (somatic) tissue, which lack a vascular connection to that tissue and which proceed through development in a process parallel to that of a zygotic embryo, which is derived from the fusion of two gametes. The zygotic and somatic embryogenic pathways are similar enough that somatic embryogenesis is often used as a proxy to study the molecular mechanism of zygotic embryo initiation (Loyola-Vargas 
2016). In addition to its use in basic scientific studies as a model for investigating embryo development following sexual reproduction, somatic embryogenesis offers a method for mass propagation of clonal plant germplasm, which is useful for replicating plants which are rare, slow to mature, or for quickly scaling up generation of plant genotypes possessing particularly desirable varietal characteristics (Guan et al. 2016). Somatic embryos can even be used to produce so-called "synthetic seeds" by encapsulating them in a gel-like medium containing stores of essential nutrients which are supplied by the endosperm during germination in normal seeds (Reddy et al. 2012). However, the greatest utility for somatic embryogenesis today is as a means of regenerating mature whole plants from cells in which DNA sequences originating from outside the target plant genome have been introduced (Elhiti et al. 2013).

\section{History of plant transformation}

Transfer of functional exogenous genetic sequences into plant genomes actually took place many millennia prior to any human intervention. It was reported only a few years ago that the plant pathogen Agrobacterium tumefaciens, which is the causative agent of crown gall disease in a wide array of plant species, facilitated at least one horizontal gene transfer event in sweet potato (Ipomoea batatas), evidence of which is retained in the genome of every one of hundreds of sweet potato accessions tested. This event was apparently selected for during sweet potato domestication as the sequence in question is not found in the genomes of the crop's wild relatives (Kyndt et al. 2015). Since then, sequences derived from Agrobacterium transferred DNA sequences have 
been discovered in the genomes and transcriptomes of dozens of plant species, suggesting that this may be a widespread phenomenon in nature (Matveeva and Otten 2019). It was not definitively shown that Agrobacterium tumefaciens causes crown gall disease by stably transmitting a DNA segment into the host plant cells until 1977 (Chilton et al. 1977). At this time, it was hypothesized that this capability could be co-opted in order to facilitate the transfer of any chosen DNA sequence into plants. This discovery launched efforts by multiple research groups to accomplish the first instance of intentional plant transformation. Production of the first transgenic plant cell lines (tobacco, petunia, and sunflower) were reported by three separate public research groups and a group at Monsanto all in 1983 (Somssich 2019) and a whole regenerated transgenic plant (tobacco) was described the following year (De Block et al. 1984).

Soon after Agrobacterium-mediated plant transformation was first achieved, other methods of DNA delivery were demonstrated. Also in 1984, a plant (turnip) was first transformed using a viral vector (cauliflower mosaic virus) (Brisson et al. 1984). DNA microinjection of protoplasts, electrophoresis, and particle bombardment all came into use in the 1980s (Fromm et al. 1986, Lawrence and Davies 1985, Sanford et al. 1987). Initially, Agrobacterium-mediated transformation was only thought to be suitable for transforming dicotyledonous plants as there are (with a handful of notable exceptions such as asparagus) very few monocots known to be susceptible to crown gall in nature, and consequently particle bombardment was more commonly employed for monocot transformation (Cheng et al. 2004, De Cleene 1985). However, alterations to transformation methodologies, including the development of new transformation-competent 
strains of Agrobacterium, have enabled successful transformation of many major monocot crops (e.g., maize, sorghum, wheat, rice) using this method (Zhang Wan-Jun et al. 2013). The ability to transform plants significantly broadens the range of traits that are available for germplasm improvement (relative to conventional breeding) by expanding the potential gene pool outside the boundaries imposed by sexual compatibility. Even genes derived from organisms in different kingdoms are candidates for transformation. In fact, several of the most widely deployed transgenic traits are conferred by bacterial genes including glyphosate tolerance conferred by CP4 EPSP synthase and insect resistance conferred by various cry genes from Bacillus thuringiensis (Kleter et al. 2005). In addition to traits that benefit growers, transformation has been used to develop crops intended to benefit the end-consumer, for instance, by enhancing a food's nutritional content. The "golden rice" project is a well-known example of this (Beyer 2010). In addition to trait enhancement, transformation capabilities have greatly benefitted basic research in genetics. It is routinely used to investigate the function of individual genes, especially in model plants such as Arabidopsis, and it has been employed to generate a massive library of mutant seeds for most genes in the Arabidopsis genome through TDNA insertional mutagenesis (Alonso et al. 2003).

Emerging biotechnological methods 
Several technologies have arisen over the last several years which hold promise for further broadening the range of traits that can be achieved through genetic manipulation. These methods are being rapidly adopted by the plant research community. However, their practical applications are often constrained by limitations in plant regeneration and transformation capabilities. Thus, the development of techniques that serve to increase the efficiency of these underlying processes, by building upon an improved mechanistic understanding of them, will be key to realizing the full potential of novel biotechnological tools. A few of these emerging technologies are described below.

Genome editing. While in conventional genetic engineering a segment of exogenous DNA is inserted into the genome at a more or less random location (Gelvin 2017), a defining feature of genome editing is that a precise site of action is selected for modification. In the simplest use-case, DNA coding for a custom engineered molecule or molecular complex with both nucleotide sequence-specific binding and nuclease functions is transformed into plant cells, and when expressed, the mature product is directed to a pre-determined genomic locus where it induces a double-stranded break in the DNA. This lesion is then mended by the cell's native DNA repair machinery, which is imperfect, and often results in alteration of the original sequence (Kumar and Jain 2015). Using this method, novel alleles may be induced in a chosen gene sequence without relying on randomness inherent in other mutagenesis techniques (Holme et al. 2019). Several molecular tools have been successfully employed in academic research to accomplish gene 
editing over last several years including meganucleases, engineered zinc-finger nucleases, and TAL effector nucleases (Christian et al. 2010, D'Halluin et al. 2008, Townsend et al. 2009), all of which suffered from various disadvantages regarding ease of use that prevented their wide adoption for crop improvement. However, the demonstration that the CRISPR/Cas9 system (a genomic feature of many prokaryotic organisms that functions in the acquisition of immunity to phage viruses) could be directed toward unique genomic sequences for cleavage using an engineered single guide RNA molecule (Jinek et al. 2012), and that this system could be heterologously expressed in eukaryotic cells for targeted mutagenesis (Cong et al. 2013), led to an explosion of interest in and applications for genome editing. Another notable advantage of genome editing relative to standard genetic engineering is that the presence of the molecular machinery used to introduce a heritable sequence alteration is no longer necessary to maintain the stability of a modified trait once the intended edit is accomplished. Therefore, if the exogenous DNA coding for the machinery is integrated into the plant genome, it may be segregated away from the edited allele or otherwise excised from the host genome in subsequent generations. This can potentially result in a plant line that carries no exogenous DNA and is indistinguishable from a plant variety produced by mutagenesis methods routinely used in conventional breeding (Miroshnichenko et al. 2019). Refinements of genome editing constructs optimized for plants and employment of variant CRISPR/Cas systems has enabled several more sophisticated methods of targeted editing other than 
gene knockout, including: single-base substitution, insertion/deletion of 5-200 bp sequence segments, targeted transcriptional activation/repression, and even targeted epigenetic modulation (Chen et al. 2019).

Gene targeting. Also referred to as knock-in, gene targeting involves the seamless insertion of a DNA sequence, long enough to encode a functional protein product, into a predetermined location within the target genome. Gene targeting relies on activation of the specific cellular pathway of homology-directed repair (HDR) to facilitate the insertion. A donor DNA template is delivered into target cells, which contains the sequence to be inserted flanked on either side by "homology arms," which are stretches of nucleotides up to a few hundred base-pairs in length each that match exactly to genomic DNA at the site where the insertion is to be made (Qi et al. 2013). The use of gene targeting pre-dates contemporary gene editing tools, but for years the efficiency has been far too low for it to be used for routine genetic modification (Puchta 2002). The employment of a site-directed nuclease to induce a double-strand break at the intended insertion site can increase the frequency of gene targeting over 10-fold, but seamless insertion events remain exceedingly rare in most applications of this method (Huang and Puchta 2019). Some recently published findings detail novel approaches to gene targeting which offer hope for improving its efficiency (Lin et al. 2020, Schiml et al. 2014, Wolter and Puchta 2019). One apparent path to enhancing gene targeting would be to selectively ensure regeneration in the relatively sparse population of cells for which HDR-mediated insertion of the target sequence is successfully induced (Fauser et al. 2012). 
In-planta transformation. The hallmark of this set of methods is the ability to completely bypass sterile tissue culture, achieving regeneration of transformed plants often much more quickly than for standard transformation methods and minimizing the risk of somaclonal variation sometimes observed in plants regenerated from tissue culture (Mayavan et al. 2013). Floral dip transformation, which is routinely used in Arabidopsis and other members of the Brassicaceae family is an example of an in-planta method. However, floral dipping is not widely applicable to plant species outside of this taxonomic group (Kojima et al. 2006). In addition to floral dipping, vacuum infiltration of $\mathrm{Ag}$ robacterium suspensions, Agrobacterium injection, pollen-tube gene transfer, and floral spray are all in-planta methods which have shown varying degrees of success in different plant species. Generally, these techniques suffer from low efficiency and a higher rate of chimerism compared to tissue culture-based methods (Niazian et al. 2017). Recently, an efficient method for in-planta transformation of genome editing constructs was reported in Nicotiana benthamiana, tomato, potato, and grape (Maher et al. 2020). This procedure utilized the induction of de novo meristems formed upon the delivery of developmental regulator (DR) genes by Agrobacterium application to wound sites of plants for which meristems had been removed. By applying the right balance of DRs, cells in which genome editing reagents were expressed were also selected for regeneration of de novo transgenic shoots. This study demonstrates that genes governing regeneration in plants can serve as a vital tool for advancing plant biotechnology, even in contexts where tissue culture is not used. 


\section{Discussion}

\section{A detailed look at the somatic embryogenesis process}

Overview of morphological and developmental characteristics of somatic embryogenesis

Unlike animals, plants possess the unique ability to regenerate entire individuals from somatic cells in a process called somatic embryogenesis (SE). This is accomplished through dramatic reprogramming of cell fate in tissue types that are receptive to the signals that trigger SE (Namasivayam 2007). SE is distinct from regeneration via de novo shoot organogenesis (DNSO) in that the clonal structures formed (somatic embryos) are bipolar, containing progenitor cells with the capacity for development of both shoots and roots, as opposed to de novo shoots which are unipolar. Also unlike de novo shoots, somatic embryos do not retain a vascular connection to the "parental" tissue they are derived from, though, similar to zygotic embryos they can be supplemented with nutrients and hormones from the subtending tissue via intercellular exchange (Von Arnold et al. 2002). Though somatic embryo formation from vegetative tissue was first conceived as it was induced under artificial conditions in tissue culture and continues to be most widely studied in this context, SE has also been documented to occur in nature. A wellknown example of this is found in plants of the tropical genus Kalanchoë, also known as "mother of thousands," which are known to form spontaneous somatic embryos on their leaf margins (Dodeman et al. 1997). Several other instances of the natural formation of asexual embryos have been noted as well (Tisserat et al. 1979). 
Somatic embryogenesis can be induced under tissue culture conditions often by treatment with exogenous auxin compounds, stress, or a combination of both (Dudits et al. 1995). Efficient induction of SE through tissue culture in a given plant species and specific genotype is not trivial and may involve extensive testing of different types of source tissue, basal media formulations, types and concentrations of plant growth regulator compounds, pre-culture treatments of tissue, and environmental factors during culture incubation in order to reproducibly achieve plantlet regeneration via this process. There remain many plants for which experimental methods suitable for inducing SE have not been reported thus far (Solís-Ramos et al. 2012). Source tissues which are excised from the plant, placed in sterile culture, and from which regeneration is induced are referred to as "explants." Explants commonly employed for SE induction are often derived from organs present at more juvenile developmental stages such as hypocotyls, cotyledons, and mature or immature zygotic embryos, though leaf disks, stem internodes, petioles or root segments are occasionally used as well (Phillips and Garda 2019). A distinction is sometimes drawn in the literature between direct and indirect SE, wherein a somatic embryo either emerges directly from the surface of cultured explant tissue or arises from a mass of amorphous tissue called "callus," which forms an intermediate stage between explant and somatic embryo (Williams and Maheswaran 1986). In practice, however, there is not such a bright line between direct and indirect SE as they can co-occur even within the same culture. Somatic embryo formation in culture may instead be conceptualized as a continuum of developmental vigor influenced by numerous factors (Horstman et al. 2017a). 
Generally speaking, the plant growth regulator type that is most important for driving SE is the master hormone auxin. The synthetic auxin compound 2,4-dichlorophenoxyacetic acid (2,4-D), which was originally developed during World War II as a powerful herbicide for targeting broadleaf weeds (and as a potential chemical warfare agent) (Peterson 1967), has been used particularly frequently in developing SE systems in a wide range of crops. In addition to its potent auxin activity, 2,4-D may help promote somatic embryogenesis by inducing a stress response in the plant (Gaj 2004). The application of other auxins such as 1-naphthaleneacetic acid (NAA) and indole-3-acetic acid (IAA) in tissue culture media may assist in somatic embryo induction in some contexts, along with cytokinins such as 6-benzylaminopurine (BAP), or occasionally, gibberellins and abscisic acid (Jiménez 2005). Once somatic embryos are established, transfer to low-auxin or hormone-free media is often required for further maturation and tissue differentiation (Yang and Zhang 2010).

When an explant is placed into embryogenic culture, only relatively few cells within the tissue are competent to receive the hormonal and stress-mediated induction signals. Such cell populations often consist of stem cell-like niches associated with organ formation in the shoot, root, or vasculature (Fehér 2015, Quiroz-Figueroa et al. 2002). Embryogenic callus tissue proliferating from explants is derived from pericycle-like cells located in various tissues throughout the plant and maintains a closely overlapping identity with the cells involved in the formation of lateral root primordia (Malamy and Benfey 1997, Sugimoto et al. 2010). Embryogenic calli are often distinguishable from non-embryogenic calli after a few days to several weeks in culture by their color and 
other morphological characteristics (Yang and Zhang 2010). Competent vegetative cells destined for somatic embryo generation proceed through multiple discernable developmental steps: I) either dedifferentiation (reversion of partially differentiated cells to a more primordial developmental state) or transdifferentiation (a lateral transition of cell fate in adult stem cells) (Fehér 2015, Sugimoto et al. 2011); II) proliferation (minimal in the case of direct SE and more extensive in indirect SE); III) acquisition of embryonic identity (totipotency); and IV) commitment to progression through the embryogenic pathway (Elhiti et al. 2013).

Both single-cell and multicellular origins for somatic embryos have been documented in different cases. Where embryos derive from a single cell, development is characterized by coordinated cell divisions as demonstrated by cell fate tracking experiments (Pasternak et al. 2002, Toonen et al. 1994) and embryos are connected to the subtending tissue only by a suspensor-like structure. When they are multicellular in origin, there is no apparent pattern in the initial cell divisions and the embryo is more closely associated with the explant or callus (Quiroz-Figueroa et al. 2006). Early embryogenic tissue is detectable by histological methods as proembryonic masses (PEMs), which are clusters of proliferating cells which have dense cytoplasm and small vacuoles compared to the majority of quiescent cells seen within the underlying tissue (Filonova et al. 2000). Somatic embryos are first identifiable on a macroscopic scale as a spherical structure emerging from the explant or callus tissue. This is referred to as the globular stage. In dicots, as the cotyledons and primordial shoot apical meristem start to develop 
the embryos transition to the heart-stage, followed by the torpedo stage and the cotyledonary stage as the suspensor-like structure elongates and the primary stem tissues begin to differentiate. As monocot embryos develop with only a single cotyledon, their morphological stages are necessarily different. The analogous stages to heart, torpedo and cotyledonary forms in dicots are transition/club, scutellar, and coleoptilar stages in monocots (Zhao et al. 2017).

Genetic basis of somatic embryogenesis

Although there is no full accounting of the molecular events and mechanisms that facilitate SE in plants, many studies have been conducted which identify genes that can influence either the capacity for SE in certain tissue culture contexts or at least can increase the rate of somatic embryo formation, and several regulatory relationships between different key players have been established.

Boutilier et al. (2002) were the first to identify the AP2-like transcription factor gene $B A B Y B O O M(B B M)$ in Brassica napus for its importance in zygotic embryo development. They found that ectopic expression of this protein in either B. napus or Arabidopsis thaliana led to spontaneous somatic embryo formation on seedlings in the absence of hormones. Since this initial work, BBM has been confirmed to control SE induction in several different plant species including tobacco, poplar, pepper, cacao, and maize (Deng et al. 2009, Florez et al. 2015, Heidmann et al. 2011, Lowe et al. 2016, Srinivasan et al. 2007). Tsuwamoto et al. (2010) demonstrated that another AP2-like transcription factor closely related to $B B M$, dubbed EMBRYOMAKER (EMK; also known 
as AINTEGUMENTA-like 5/AIL5), was also able to promote generation of somatic embryo-like structures in culture, though these displayed pleiotropic effects and were not able to mature past an embryonic stage. Later, Horstman et al. (2017b) showed that there was at least partial functional redundancy between $B B M, E M K / A I L 5$, and several other genes forming a subclade within the AP2/ERF superfamily including PLETHORA 1 (PLT1), PLT2, PLT3/AIL6, and PLT7 through generation and testing of transgenic overexpression lines for each. This study also determined that the effectiveness of $B B M$ and related genes in inducing cellular totipotency is dose- and tissue context-dependent.

Overexpression of the MADS-box transcription factor AGAMOUS-LIKE15 (AGL15), has shown the ability to boost the rate of somatic embryo generation in cultures of Arabidopsis and soybean and to promote embryogenic callus formation in cotton (Harding et al. 2003, Thakare et al. 2008, Yang et al. 2014), though unlike BBM, it has not been shown to be capable of inducing SE in contexts where the process is not already present to some degree (Gordon-Kamm et al. 2019). Several auxin signaling transcriptional corepressor genes of the AUXIN RESPONSIVE/INDOLE-3-ACETIC ACID INDUCIBLE (AUX/IAA) family have also been demonstrated to be involved in SE. These include IAA16, IAA29, IAA30 and IAA31 for which Arabidopsis T-DNA insertional single mutant lines of each were significantly impaired in SE induction compared to wild type plants (Gliwicka et al. 2013). Furthermore, it was shown that IAA30 is a direct target of transcriptional regulation by AGL15 (Zheng et al. 2009).

LEAFY COTYLEDON 1 (LEC1), encoding a subunit of nuclear factor complex $Y$, was first determined to increase the formation of somatic embryos when it was ectopically 
expressed during the late stages of embryogenesis in Arabidopsis (Lotan et al. 1998). It had been previously implicated in zygotic embryo development and in controlling seed dormancy (Parcy et al. 1997). Kalanchoë daigremontiana, a plant known for its ability to constitutively generate somatic embryos on its leaf edges, was found to have an aberrant (among consensus sequences in other plant clades) LEC1 gene that encodes a truncated protein product. When a chimeric, non-truncated version of the $L E C 1$ ortholog was expressed transgenically in $K$. daigremontiana under the control of the A. thaliana LEC1 promoter, the spontaneous somatic embryos developed seed dormancy characteristics, indicating that this variation in the $L E C 1$ gene is what allows this unique propensity for clonal reproduction (Garcês et al. 2014). The LEAFY COTYLEDON 2 (LEC2) gene, which is unrelated to $L E C 1$ and instead codes for a B3 family transcription factor, was also shown to participate in both zygotic and somatic embryogenesis in Arabidopsis (Stone et al. 2001). LEC2 is expressed earlier than LEC1 in embryo development and it has been shown to differentially regulate transcripts of other genes implicated in SE including AGL15 and IAA3O along with the auxin biosynthesis genes YUCCA2 and 4 (YUC2/4) (Braybrook et al. 2006, Stone et al. 2008). In tobacco, inducible expression of LEC2 is sufficient to cause somatic embryogenesis, but inducible expression of $L E C 1$ is not (Guo et al. 2013).

LEC1, the similarly characterized LEC1-like (L1L), along with other transcription factor genes ABSCISIC ACID-INSENSITIVE 3 (ABI3), FUSCA3 (FUS3), and LEC2, are recognized to participate in a co-regulatory network, termed the "LAFL" network, which controls the germination and maturation process in early plant development (Jia et al. 
2014). In Arabidopsis, lec1, lec2, and fus3 mutants are strongly impaired in SE. The process is completely abolished in double and triple mutants of these genes (Gaj et al. 2005). The regulation of genes in the LAFL network in the context of somatic embryogenesis was yet another avenue of investigation reported in Horstman et al. (2017b). $B B M$ was shown to transcriptionally regulate each of the LAFL genes in Arabidopsis. Though, again $L E C 2$ had been shown to regulate $A G L 15, B B M$ did not directly transcriptionally regulate $A G L 15$ at the SE stage examined, even though it had been shown to bind the AGL15 promoter in a ChIP-seq experiment (Horstman et al. 2015).

The homeodomain transcription factor WUSCHEL (WUS) was first known in Arabidopsis for its role in the maintenance of floral and shoot apical meristems (Laux et al. 1996). As with BBM, ectopic expression of WUS, provided that it was not expressed constitutively, led to somatic embryo induction even in contexts where no SE occurred in the wild type plants (Zuo et al. 2002). However, WUS was unique in that its role in maintaining stem cell niches in the shoot allowed it to induce the formation of de novo shoot meristems, allowing shoot organogenesis to take place within non-shoot tissue (Gallois et al. 2004). WUS has proven to be useful for promoting SE in crop plants such as coffee, chili pepper, white spruce, and cotton (Arroyo-Herrera et al. 2008, Bouchabke-Coussa et al. 2013, Klimaszewska et al. 2010, Solís-Ramos et al. 2009). WUS functions in the latter steps of the SE process, as developing embryos reach the globular stage, to establish an organizing center at the newly differentiating apical meristem through repression of type A ARABIDOPSIS RESPONSE REGULATOR genes, which are themselves negative regulators of cytokinin signaling (Leibfried et al. 2005). WUS is itself induced by auxin, though 
since its expression is strictly spatially regulated in order to form a functional meristem, this is likely controlled by directional transport of endogenous auxin rather than by an exogenous auxin source such as 2,4-D (Su et al. 2009). Relatives of WUS, referred to as the WUSCHEL-RELATED HOMEOBOX (WOX) genes, also play various roles in SE. WOX2 and WOX8/9 function in the very earliest part of embryogenesis, differentiating the apical cell from the basal cell after the first division (Haecker et al. 2004). Parallel to the function of WUS at the shoot apex, WOX5 plays a role in organizing the quiescent center of the root apex and is important in the establishment of that meristem (Wang and Chong 2016). A homolog of WOX9 has been recently reported to induce SE in Medicago truncatula (Tvorogova et al. 2019).

Plant developmental patterns and tissue differentiation are ordinarily maintained through tight epigenetic regulation of the chromatin state at the loci of key developmental genes. For development to progress, critical genes involved in the maintenance of an embryonic state, including BBM and the $L A F L$ network, as well as stem cell regulators like WUS, WOX5, and SHOOT MERISTEMLESS (STM) can be restrained by histone modifications conferred by Polycomb Repressive Complexes 1 and 2 (PRC1 and 2) (Chen et al. 2010, Ikeuchi et al. 2015, Liu et al. 2016, Yang et al. 2013). These multiprotein epigenetic regulator complexes are conserved across plants and animals. PRC1 functions in silencing gene expression by ubiquitination of histone 2A, while PRC2 cataIyzes trimethylation of H3K27. the closely related B3 transcription factors VIVIPAROUS1/ABI3-LIKE 1 and 2 (VAL1/2) can interact with other B3 proteins of the LAFL network and are able to recruit at least PRC1 to LAFL gene loci to sustain their expression 
(Yang et al. 2013). VAL1 and 2 can also mediate repression of these embryonic genes through specific interactions with and recruitment of histone deacetylase proteins encoded by HDA6 and HDA19, respectively (Chhun et al. 2016, Zhou et al. 2013b). Another epigenetic modulator involved in repressing the genes involved in SE is the CHD3 chromatin-remodeling factor PICKLE (PKL), for which mutant plants show decreased H3K27 trimethylation at the LEC1 and LEC2 loci (Zhang et al. 2008). Horstman et al. (2017b) showed that $B B M$-mediated SE was enhanced in both the val1-2 and pkl-1 mutant backgrounds, suggesting that they each function in suppression of the BBM SE pathway either through silencing of $B B M$ itself or one of its downstream targets.

A cellular signaling component, which was named SOMATIC EMBRYOGENESIS RECEPTOR KINASE 1 (SERK1) was shown to be highly expressed during early embryogenesis in A. thaliana. Constitutive overexpression of this gene leads to an enhancement of somatic cell competence for embryogenic transition (Hecht et al. 2001). Other genes loosely associated with a stress response, which also influence SE include non-symbiotic hemoglobins, of which the maize homolog of GLB2 promotes SE when it is suppressed through its control of programmed cell death (Huang et al. 2014), germin-like proteins in conifer SE whose functional roles are not fully characterized (Mathieu et al. 2006), and reactive oxygen species (ROS)-scavenging enzymes such as ascorbate peroxidase (APX) and glutathione S-transferase L3 (GSTL3) genes which, when suppressed, accelerated the process of redifferentiation in embryogenic calli of cotton (Zhou et al. 2016). Several other genes that influence regeneration in plants, including by SE, through wound signaling are reviewed in Lup et al. (2016). 
Some recent research has yielded insight into the role of small RNA in SE. One study (Szyrajew et al. 2017), found that $64 \%$ of the $A$. thaliana genes giving rise to primary miRNA transcripts were differentially regulated during embryogenesis. Wójcik et al. (2017) showed that miR160 and miR166/165 help control embryogenesis through the post-transcriptional regulation of $A R F$ genes and HD-ZIPIII transcription factors $P H B$ and PHV, respectively. Lin et al. (2015) determined that trans-acting short interfering RNA (tasiRNAs) derived from the TAS3 transcript regulates the expression of ARF3 and ARF4 during embryogenesis in Dimocarpus longan. Recent reports have identified additional small RNA players in SE through differential analysis of global small RNA expression in maize and coconut (Alejandri-Ramírez et al. 2018, Sabana et al. 2020). Small RNA interference technology offers exciting prospects for promoting somatic embryogenesis in recalcitrant plants.

From the diversity of hormonal treatments or physical manipulations that can induce somatic embryogenesis in plants such as Arabidopsis and tobacco, it appears that there may be multiple entry points into the initiation of this process. Though several genes and molecular interactions have been well-established as contributing to somatic embryo formation, development, and maturation, many gaps remain in the mechanistic understanding of SE. It remains to be seen whether all the known molecular players described so far act together as a module forming a coordinated somatic embryogenesis pathway, whether there are different independent mechanisms whereby SE can occur, or to what extent either a unified pathway or multiple independent pathways might be evolutionarily conserved across the plant kingdom. 
A detailed look at Agrobacterium-plant interactions surrounding transformation Overview of Agrobacterium biology, pathogenesis, and DNA transfer mechanism

Agrobacterium tumefaciens is a Gram-negative soil-borne bacterium that is the causative agent of crown gall disease, which affects a wide range of host species (DeCleene and DeLay, 1976). Through the course of its infection of a host plant, Agrobacterium mobilizes a single-strand DNA segment originating from a sequence located on its tumor-inducing (Ti) plasmid, referred to as transfer DNA (or T-DNA), into host plant cells (Chilton et al. 1977). Incorporation of T-DNA into the genome of the host and the subsequent expression of the gene products it codes for leads to the formation of tumorous growths that are characteristic of crown gall disease (Escobar and Dandekar, 2003). This uncommon ability to transmit DNA sequences into hosts and modify their gene expression as part of its infection strategy has made Agrobacterium an important tool in the development of transgenic plants for crop breeding and basic research through the utilization of a method known as Agrobacterium-mediated transformation (Azpiroz-Leehan and Feldmann, 1997). The close contact between pathogen and host throughout the different stages of infection gives rise to heavily intertwined and multi-layered molecular interactions among them. The successful genomic integration and expression of T-DNA in a host is dependent on a few different steps occurring during pathogenesis including signal perception, expression of pathogen virulence genes and T-DNA processing (the presence of a Ti plasmid is required for transfer of T-DNA), attachment of Agrobacte- 
rium to the host cell, transport of T-DNA and accessory proteins into the host cell, nuclear import, and finally uncoating of the T-DNA strand and recombination with the host genome (Gelvin 2000). Molecular interactions at any one of these stages can affect the success of Agrobacterium in infecting the plant. To attempt to understand these interactions, we need to examine each stage in greater detail.

In nature, the infection process begins with the production of phenolic compounds by a potential host. This may be caused by environmental stress or damage due to wounding. Perception of plant-derived phenolic molecules can induce the expression of a suite of genes known as the vir regulon, which is housed on the Ti plasmid (Bhattacharya et al. 2010). The Agrobacterium cell attaches to the surface of the host plant, mediated by pathogen-secreted polysaccharide and protein adhesin compounds. Following attachment, Agrobacterium cells becomes sessile. Although the Ti plasmid is required for T-DNA transfer and tumorigenesis, it is not strictly required for this polar attachment process (Tomlinson and Fuqua 2009). It is at this stage that the plant has its first opportunity for defense against Agrobacterium infection. Some bacteria-derived compounds known as pathogen-associated molecular patterns (PAMPs) may be perceived by the host, thereby invoking a quick, but relatively weak immune response termed PAMP-triggered immunity (PTI) (Zipfel and Robatzek 2010). The most well-studied Agrobacterium-derived PAMP that elicits a PTI response is EF-Tu, which was found to be recognized by the host receptor kinase protein EFR. In addition to EF-Tu, other yet to be characterized PAMPs may play a role in the molecular interactions at this stage (Zipfel et al. 2006). 
Provided that a virulent strain of Agrobacterium has undergone attachment to the plant surface and the expression of vir genes has been induced, the T-DNA segment on the Ti plasmid is nicked at right and left border sequences and becomes covalently attached to a VirD2 protein molecule at the $5^{\prime}$ end (Mysore et al. 1998). The T-strand is shuttled through a type IV secretion system into the cytoplasm of a host plant cell where it is thought to be coated by molecules of the VirE2 protein (Ziemienowicz et al. 2001). Mutually bound VirD2, single-stranded T-DNA, and VirE2 are believed to form what is termed the "T-complex" (Vergunst et al. 2000). In addition to VirD2 and VirE2, other virulence proteins are known to be delivered into the host cell including VirE3, VirD5, and VirF. (Thompson et al. 1988, Ward et al. 1988, 2002, Kuldau et al. 1990, Shirasu et al. 1990, Beijersbergen et al. 1994, Christie and Vogel 2000, Schrammeijer et al. 2003). VirE2 contains a plant-active nuclear localization signal and has been shown to interact with the plant protein VIP1, which was initially thought to be involved in T-DNA integration into the genome (Tzfira et al. 2001, Djamei et al. 2007), however, its participation in this process has been disconfirmed in more recent studies (Shi et al. 2014, Lapham et al. 2018). The T-DNA from wild-type strains of Agrobacterium encodes the plantactive genes $i a a H, i a a M$, and ipt, which induce the biosynthesis of auxin and cytokinin. The expression of these genes gives rise to the formation of crown galls (Morris 1986, Binns and Costantino 1998). For the purpose of adapting T-DNA transfer for biotechnological applications, Agrobacterium strains which have had their Ti plasmids "disarmed" (i.e., non-tumorigenic) are used (Barton and Brill 1983). This means that the oncogenes present in the T-DNA segment of the plasmid have been deleted whereas the vir genes 
remain. Therefore, non-tumorigenic strains of Agrobacterium often used in the lab still transfer proteins into host cells via a type IV secretion system, but do not lead to the formation of crown galls.

Bacterial pathogens other than Agrobacterium are known to transfer proteins into the plant cell during infection, which may serve to dampen the PTI response. However, host plants are often able to perceive these foreign proteins (termed effectors), thereby initiating a strong defense response called effector-triggered immunity (ETI) (Cui et al. 2009). This series of defenses includes the hypersensitive response (HR) consisting of rapid programmed cell death, which prevents the spread of the disease to uninfected tissue. However, Agrobacterium is able to avoid eliciting HR in most plants despite its transfer of foreign Vir proteins. This is a key advantage Agrobacterium has over other plant bacterial pathogens (Pu and Goodman 1993, Staskawicz et al. 1995, Wood et al. 2001). Although the secreted Vir proteins seem not to elicit an immune response, they have been shown in several cases to have in planta functions and thus have the potential to modulate host gene expression patterns (Tzfira et al. 2004, Lacroix et al. 2005, García-Rodríguez et al. 2006, Magori and Citovsky 2011, Wang et al. 2014, Niu et al. 2015, Zhang et al. 2017). In order to examine the differences between the effects caused by Agrobacterium PAMPs or other non-transferred factors and those caused by the in planta activities of the Vir proteins, some of the studies reviewed here utilized "cured" strains of Agrobacterium, meaning that they no longer contain a Ti plasmid and thus cannot express or transfer Vir proteins (Watson et al. 1975). Strains lacking Ti plasmids are often referred to in the literature as "avirulent" in contrast to "virulent" strains 
that contain a disarmed Ti plasmid (Veena et al. 2003). Both of these are considered non-tumorigenic as they cannot induce crown gall formation. To date, many functions of the Agrobacterium genes involved in the pathogen-host interaction are known and most of the Vir proteins have been well-characterized (Tzfira and Citovsky 2006). By contrast, many of the host plant factors that are directly involved in the transformation process, including attachment, pathogen recognition, T-DNA transfer, trafficking through the cell cytoplasm and integration of T-DNA into the host genome remain unidentified (Gelvin 2010, 2017, Lacroix and Citovsky 2013).

Using differential gene expression to examine host genes interacting with Agrobacterium infection

One of the many approaches that have been implemented to understand the molecular players and large-scale processes involved in transformation is differential gene expression analysis. The basic premise behind this analysis is that by contrasting the transcript levels of genes between two different conditions or treatments, one can elucidate which genes are altered by the treatment and thus might be involved in facilitating or suppressing a given process. The technology for evaluating transcript expression has advanced exponentially over the past 20 years, resulting in drastically decreased cost and labor as well as increased capacity for data generation. Each of the techniques that have been developed generally involves the production of a cDNA pool generated by reverse transcription of total mRNA extracts from plant tissues. The exper- 
imental methodologies utilized in the studies reviewed here include cDNA-AFLP (amplified fragment length polymorphism), (reverse transcription) RT-PCR, suppression subtractive hybridization, DNA macroarrays, microarrays, quantitative real-time RT-PCR, and RNA-seq. For a detailed review on these methods and more, see Casassola et al. (2013). The widespread use of microarrays and RNA-seq has led to the accumulation of massive amounts transcriptional data as there can be coverage over nearly all of the genome in one experiment, yielding hundreds or thousands of differentially expressed genes. As a result, it has become popular to analyze the data generated by such experiments by classifying them according to the probable molecular or biochemical function, biological process, or cellular compartment of their predicted gene product using tools such as Gene Ontology (GO), MapMan, and the Kyoto Encyclopedia of Genes and Genomes (KEGG). This analysis serves to give an overall picture of systems in the organism that are altered, dependent on the experimental treatment.

Here, the reported findings on the gene expression dynamics of plants during Agrobacterium-mediated transformation or upon heterologous expression of Agrobacterium-derived virulence proteins in the host are reviewed. The relative benefits and drawbacks of using modern transcriptomic methodologies to examine Agrobacteriumplant interactions are also discussed.

Transcriptomic analysis in model plant systems. Several studies have been carried out over the last 20 years that utilized different methods for detection of differential gene 
expression in response to inoculation with Agrobacterium. In a few cases they have attempted to untangle the unique responses relating to particular steps of Agrobacterium infection and T-DNA transfer by various means. These include contrasting Agrobacterium response with that of other bacterial organisms, contrasting the responses to different Agrobacterium strains that are necessarily arrested at some point in the infection process owing to their genotype (including using tumorigenic versus non-tumorigenic strains), and temporally analyzing different infection stages during a time-course. Other important differences exist among these studies with respect to plant species/genotype, tissue type sampled, culture conditions, inoculation method, Agrobacterium strain(s) used, and experimental design, which could account for some of the variability in results. Therefore, these details will be thoroughly outlined for each study.

In one of the earliest studies on the plant response to Agrobacterium (Ditt et al. 2001), researchers used a cell suspension culture of the tropical plant Ageratum conyzoides to construct a cDNA library of 16,000 sequences and then implemented cDNAAFLP to identify CDNA sequences that were differentially regulated in response to the non-tumorigenic Agrobacterium strain EHA105. A total of 179 unique gene fragments were upregulated in response to Agrobacterium, whereas 72 were downregulated. Twenty of the most strongly induced of these sequences were used as queries to search for sequence similarity to other species. Top gene candidates in these similarity searches were involved in cellular functions such as signal perception, signal transduction, and defense. Using RT-PCR as an independent gene expression method, it was 
shown that four of the genes predicted to function in defense response were also induced by treatment with non-pathogenic $E$. coli cells whereas two genes, one encoding a protein similar to a nodulin from Oryza sativa and one encoding a protein similar to a lectin-like protein kinase from Populus nigra, showed an Agrobacterium-specific response. This same research group later used the same experimental system to evaluate the expression of three of the putative defense genes identified in this study in response to the attachment of Agrobacterium to the host (Ditt et al. 2005). Plant cell cultures were infected with four different non-tumorigenic strains of Agrobacterium: EHA105 harboring T-DNA vector pBISN1 (T-DNA transfer competent), LBA4404 (carrying a disarmed Ti plasmid, but no T-DNA), A136 (avirulent; no Ti plasmid) and chvB (contains an oncogenic Ti-plasmid but is an attachment-deficient mutant). RT-PCR revealed that the ability of the Agrobacterium to attach to host cells had a negative effect on the expression levels of the three defense genes being measured compared to the attachment deficient strain, which induced expression levels similar to that of the non-pathogenic bacterial control. The authors suggested that exopolysaccharide (a product of the mutated gene in $c h v B$ ) may play a role in suppressing the plant defense response.

In yet another published study by this group, the authors used a microarray to analyze wide-scale gene expression in suspension cell cultures of the Arabidopsis thaliana Ler-0 ecotype in response to infection by the tumorigenic Agrobacterium strain A348 along a time-course (Ditt et al. 2006). Although this study used a 26,000-oligonucleotide array, surprisingly, no statistically significant differentially expressed genes were found at the first three time-point comparisons with the mock control $(4,12$, and 
24 hours post-inoculation). In the comparison for the samples collected 48 hours after infection, 303 differentially regulated genes were identified that showed consistency among the four experimental replications. Of these, 115 were upregulated and 188 were downregulated. GO biological process category analysis was performed on these two gene sets. The authors reported that genes falling into the "cell organization and biogenesis" and "protein metabolism" terms were overrepresented among the downregulated genes and "electron transport or energy pathways", "response to abiotic or biotic stimulus", and "response to stress" were overrepresented among the upregulated genes. They also compared their microarray data with other publicly available data sets evaluating the transcriptomic response of Arabidopsis to various other plant pathogens and to treatment with auxin compounds. Of these comparisons, the one that had the most overlapping differentially expressed genes with the Agrobacterium data set (at 53 genes) was one from mature Arabidopsis leaves that had been infiltrated with Pseudomonas syringae (Tao et al. 2003).

Working in a tobacco BY-2 suspension cell culture system, researchers in a 2003 study used suppression subtractive hybridization and macroarrays to determine changes in gene expression in host cells inoculated with Agrobacterium (Veena et al. 2003). Cells were exposed to both virulent and avirulent non-tumorigenic Agrobacterium strains with or without the capability to transfer Vir proteins or to transfer T-DNA containing a GUS-intron reporter gene. They found that a suite of genes related to defense responses including glutathione-S-transferases and alcohol dehydrogenase were induced in the earlier time-points following infection by Agrobacterium, regardless of its ability to 
transfer T-DNA. However, during the later time-points the relative expression of these genes was higher among cells infected by the avirulent Agrobacterium strain. Another interesting finding was that genes associated with cell division and plant growth such as the core histone gene family members encoding $\mathrm{H} 2 \mathrm{~A}, \mathrm{H} 2 \mathrm{~B}, \mathrm{H} 3$ and $\mathrm{H} 4$ along with ribosomal proteins were increased in their expression levels by exposure to the virulent strain at the later time-points after infection significantly above the levels induced by the avirulent strain at the same stage of infection. This timing coincided with the earliest point that expression of T-DNA could be detected in protoplasts, suggesting that these genes may play some important role in T-DNA integration specifically.

A study using mature Arabidopsis thaliana plants examined the changes in both gene expression and phytohormone accumulation in response to infection by the tumorigenic Agrobacterium strain C58 or by the non-tumorigenic strain GV3101 (Lee et al. 2009). The lower part of inflorescence stalk of plants was inoculated just above the basal leaves. This location was chosen in order to allow the formation of crown gall tumors. Using microarray analysis to sample transcript levels at three different time-points ( 3 hours and 6 days for both strains, and 35 days post-inoculation for the tumorigenic strain only), they found that over four times as many Arabidopsis genes were significantly altered in their expression levels upon exposure to the tumorigenic Agrobacterium strain as were altered by the disarmed strain using wounded, but uninoculated, inflorescence stalks as a control. It was found that both strains induced genes falling into the MapMan functional category of "stress", while hormone-related genes were affected by both, but with a stronger response to strain C58. Genes of functional classes 
associated with changes in host morphology were activated only by exposure to C58, but not by GV3101.

A recently published study that was conducted in our lab used next-generation sequencing to characterize the changes to the whole transcriptome through time in Arabidopsis thaliana seedlings infected with either of two different non-tumorigenic strains of Agrobacterium: strain At804 (virulent) or A136 (avirulent). Seedlings were sampled across a time-course from 0 to 48 hours after infection (Duan et al. 2018). As was found in the two studies previously discussed in this section, substantially more genes were significantly altered in their expression levels by exposure to the T-DNA transfer competent bacterial strain than by the avirulent strain relative to a mock treatment. Because a greater number of transcripts can be detected and there is a greater sensitivity of measurement using RNA-seq compared to the older differential gene expression techniques, we were able to find many more genes with altered expression levels than had been reported in previous studies. Using Gene Ontology (GO) enrichment analysis, we found that categories such as "cell wall organization or biogenesis", "DNA replication", and "external encapsulating structure organization" were all overrepresented among downregulated genes and that "defense response", "response to stress", and "response to reactive oxygen species" were overrepresented among upregulated genes in response to treatment with both strains. This agrees with the previous findings that indicate Agrobacterium seems to generally repress normal plant growth and cell division and to activate defense response pathways (at least in the early stages of infection) irrespective of its ability to transfer T-DNA and Vir proteins to the host. We found that in the latter 
time-points following infection that certain functional categories were unique to treatment with one strain or the other among upregulated genes. For instance, "cellular response to stress" and "secondary metabolite biosynthetic process" were found only in upregulated genes from the avirulent treatment, indicating that these responses may be attenuated by transfer of Vir proteins and/or T-DNA. On the other hand, categories having to do with cell growth, transcription and RNA metabolism, as well as "heterocycle metabolic process" and "response to abscisic acid", were found in upregulated genes only in the virulent treatment condition. It was suggested in Veena et al. (2003) that genes associated with cell division and growth processes were induced by successful $\mathrm{Ag}$ robacterium-mediated transformation. The results of our recent study support this conclusion along with suggesting other biological and metabolic processes that may be activated specifically by vir genes or T-DNA.

Transcriptomic analysis in crop species. The majority of gene expression studies that have been used to uncover the interaction between Agrobacterium and host plants have been carried out using Arabidopsis thaliana and, to some extent, tobacco suspension cell cultures. However, there have been three studies published within the last few years that have made use of transcriptomic data to examine the effect of Agrobacterium on economically important crop species. In each of these studies, transcriptome profiling by microarray or RNA-seq was one experimental approach of several that were used to gain insight into overcoming a crop plant's recalcitrance to transformation. 
In Tie et al. (2012), the researchers used microarrays to measure gene expression throughout infection by Agrobacterium strain EHA105 in the two rice cultivars 'Nipponbare' (Nip) and 'Zhenshan 97' (ZS), representing Oryza sativa ssp. japonica and ssp. indica, respectively. Representatives from both subspecies were selected for comparison because indica varieties of rice are overall much more resistant to transformation than are japonica varieties. Embryogenic calli from either variety were sampled at 0, 1, 6,12 , and 24 hours after inoculation. The highest number of differentially regulated genes occurred at the 1 hour or 6 hour time-points, and of all unique locus identifiers that were differentially expressed in either type of callus over time (11,105 sequences) only $35 \%$ were shared between the two callus cultivars. GO enrichment analysis found some differences in biological process terms between the callus types when contrasted at the same time-point. Genes involved in "defense response" and "response to biotic stimulus" were overrepresented among the set of upregulated genes in the indica callus variety. Meanwhile, categories relating to "cell cycle", "cell division" and "DNA repair" were overrepresented among the downregulated genes in ZS callus at the earliest timepoints. Some genes that are involved in ubiquitin-proteasome degradation were repressed in the more recalcitrant variety, leading the authors to suggest that degradation of the proteins coating the T-complex may be inhibited in ZS, giving rise to a deficiency in T-DNA integration and lowering the overall transformation frequency.

Another study combined transcriptomic analysis (using RNA-seq) and proteomics (2-DE and MS) to uncover Agrobacterium-host interactions in transformed wheat immature embryo tissue (Zhou et al. 2013). Embryos of the Chinese commercial wheat variety 
'Yangmai12' were extracted from immature seed and pre-cultured for 4 days before being transformed with the non-tumorigenic Agrobacterium strain C58C1. Tissue samples were collected 36 hours following inoculation. In total, the researchers found 4,889 genes that showed significant differential expression compared with the mock treatment. GO biological process term analysis showed categories such as "chromatin assembly or disassembly", "signal transduction", "biosynthesis of secondary metabolites" and "phenylpropanoid biosynthesis" were overrepresented among these genes. The molecular function terms showed that a large portion (20.5\%) of the functionally classified genes fell into the "nucleic acid binding" category. The proteomic analysis uncovered 90 differentially expressed proteins (DEPs) between the two conditions. Notably, only 24 of these DEPs corresponded to gene sequences found in the transcriptomic data. Of these overlapping DEPs, half of them were predicted to play a role in response to stress or immunity. The differences observed between the results of the transcriptomic and proteomic analyses in this study demonstrate that measured mRNA levels do not perfectly correspond to final expression of a gene product.

In a study aimed at uncovering the mechanism of the observed improvement of Agrobacterium-mediated transformation in soybean by employment of sonication and $\alpha$ -aminooxyacetic acid (AOA), RNA-seq was used to monitor transcriptomic changes in response to Agrobacterium infection combined with sonication of the explant tissue and media amended with AOA or to Agrobacterium infection without these additions to the protocol (standard Agrobacterium transformation) (Zhang et al. 2016). Cotyledonary nodes of the soybean genotype 'Jidou17' were infected with Agrobacterium EHA105 
with or without sonication plus AOA during inoculation and samples were collected five hours after infection. As in the previous studies, plant defense and immune responses were upregulated dependent on Agrobacterium infection. A total of 2,158 differentially expressed genes (55.1\% of them upregulated) were responsive to standard Agrobacterium transformation in contrast to mock treatment, whereas 5,062 genes showed a difference in expression between standard transformation and transformation plus sonication and AOA with $69.6 \%$ of these representing downregulated genes. Upregulated and downregulated genes were categorized by their pathway function using KEGG. Interestingly, this study showed that in addition to defense genes related to PTI (which Agrobacterium has been long understood to stimulate) " $\mathrm{R}$ " genes known for participating in ETI such as RPM1, RPS2, RPS5, RIN4, and PBS1 were also induced. This is notable as Agrobacterium has not previously been shown to induce ETI-like responses. Consistent with the role of AOA as an inhibitor of phenylpropanoid biosynthesis, the authors showed that genes involved in this metabolic process are upregulated by Agrobacterium treatment (in agreement with the study discussed above), but their expression is ameliorated by sonication and AOA supplementation in tissue culture following transformation.

Transcriptional response to heterologous expression of vir genes in host plants. There have been two studies published so far that used transgenic Arabidopsis thaliana plants expressing Agrobacterium-derived vir genes in order to examine differences in gene ex- 
pression compared with wild-type plants. In Niu et al. (2015) the investigators transformed Arabidopsis Col-0 plants with the virE3 gene sequence under the control of a tamoxifen-inducible promoter. The VirE3 protein had been previously implicated as a possible plant-active transcription factor because it could induce transcription in yeast when fused with a DNA-binding domain (García-Rodríguez et al. 2006). Fourteen-dayold seedlings of these virE3 transgenic plants were treated with tamoxifen to induce transgene expression and RNA-seq was used to profile the differential gene expression compared to wild-type and mock treated transgenic plants. They found 607 genes that were upregulated and 132 that were repressed specifically by virE3 expression (using a fold-change cutoff of 3 or 0.33 ). Among the upregulated genes were those encoding the plant protein VBF (a functional homolog of the Agrobacterium VirF protein), which had been previously shown to be induced by Agrobacterium infection and to play a role in destabilizing VirE2 (Zaltsman et al. 2010). Another gene that they found to be highly induced by VirE3 was that coding for NIMIN1, which binds the salicylic acid signaling protein NPR1 and reduces expression of the pathogenesis-related $(P R)$ genes. (Weigel et al. 2005). They showed through additional experiments that the promoters of both VBF and NIMIN1 could be bound by VirE3. The authors performed GO term enrichment analysis on their datasets of differentially expressed genes. Biological process categories including "signal transduction", "response to stress", "DNA-dependent transcription" and "developmental processes" were overrepresented among upregulated genes in tamoxifen-induced VirE3 plants. Examples of the molecular function categories that were highlighted are: "Kinase activity", "Transporter activity", and "Transcription factor activity". 
Duan et al. (2018) produced transgenic virE3 as well as virE2 Arabidopsis Col-0 plants with their expression under the control of the CaMV 35S promoter. These plants were tested for expression of virE3 and virE2 mRNA, respectively, using qRT-PCR. As determined by qRT-PCR, for both transgenic plants the expression levels of defense genes, including $P R 3, C R K 41$, and $C R K 18$, were elevated compared to wild-type controls upon treatment with the avirulent (cured) Agrobacterium strain A136. Meanwhile the defense genes $F R K 1, P R 2$, and $P R 4$ showed increased expression only in the virE3-overexpression plants. These limited gene expression data can be interpreted to indicate that overexpression of VirE2, and to a greater extent VirE3, enhances the plant defense response to Agrobacterium, though an alternative interpretation is that constitutive expression of the virulence transgenes has interrupted the normal functioning of the defense pathways in the plant.

Heterologous expression of vir genes in plants is an attractive method for investigating their specific functions as it allows the gene expression changes they induce in the host to be decoupled from expression changes caused by the presence of the Agrobacterium itself (i.e., defense responses due to induction of PTI). It would be far more difficult to evaluate gene expression from the same angle using modified Agrobacterium strains to induce gene expression changes. However, there are necessarily caveats when attempting to compare the expression of a virulence gene in the host cell to what actually occurs during the infection process. For example, delivery of the molecule from $\mathrm{Ag}$ robacterium to the host may be precisely controlled, and thus expression levels of the 
transgene from the plant may not correlate with the level of protein that occurs in Agrobacterium-mediated transformation. It is also possible that differential expression of some genes may represent secondary transcriptional effects following induction of the virulence gene, which do not represent the normal function of the vir gene during TDNA transfer. Taking this into consideration, it is obviously advantageous to design transgene cassettes using an inducible system such as that used in Niu et al. (2015) rather than constitutive expression. This way, at least the timing of expression can be controlled, potentially allowing for temporal isolation of primary transcriptional effects from those of more indirect responses. The Arabidopsis genes VBF and NIMIN1, which Niu et al. (2015) demonstrated could be transcriptionally regulated by VirE3, were both included in a set of genes of upregulated in response to both tamoxifen-induced VirE3 expression and treatment with virulent Agrobacterium strain At804. Surprisingly, however, both of these genes were almost equally upregulated in seedlings treated with either At804 or the avirulent strain A136 relative to the mock treatment (Duan et al. 2018). This result suggests either that some Agrobacterium-derived PAMP, or at least an extracellular signal, initiates a VirE3-independent route by which these genes can be induced, or that VirE3 is dispensable not only for overall T-DNA transfer efficiency, but also for its role in transcriptional activation, and thus is functionally redundant with some host protein. This question could be resolved by evaluating the expression levels of these genes in plants treated with a virE3 mutant Agrobacterium strain.

\section{Conclusion}


Advantages and limitations of transcriptomic analysis as an approach to discover plant genes and pathways associated with somatic embryogenesis and Agrobacterium-me-

\section{diated transformation}

There are obvious advantages to employing a transcriptomic approach for the purpose of gene discovery and pathway elucidation. Transcriptome-wide analysis allows for a quick survey of nearly all expressed genes in a given organism under specific conditions at a relatively low cost. The assays used are very sensitive and can give an accurate reflection of gene expression levels at specific times. Relative to older techniques, these methods also have a high degree of reproducibility. The advent of next-generation sequencing technology has made transcriptomic analysis the preferred choice for gene discovery and, as discussed above, it has been instrumental for uncovering the molecular players in specific pathways such as those involved in somatic embryogenesis or the Agrobacterium-mediated transformation process. Some of the main reasons for the rapid adoption and wide use of RNA-seq include the low background signal, its capability for detecting a wide dynamic range of expression, and its generation of novel sequence data at a single-base resolution. Unlike microarray technology, it is not reliant on preexisting sequence information and is not susceptible to false signals generated by potential cross-hybridization of similar transcript species to the same sequence probes. All of this allows a much greater amount of much higher quality data to be generated than was previously possible for gene expression studies.

As a tool for gene discovery, transcriptomic analysis has intrinsic limitations. The sequences detected by these methods, after all, reflect only mRNA transcripts and not 
the final products of the respective gene's expression (i.e., protein or metabolites).

Thus, a survey of the transcriptome is blind to the effect of translational or post-translational controls on gene expression. Another drawback to RNA-seq is that the amount of data generated can be overwhelming and requires significant virtual storage space and some specialty to manage and analyze the data. Too much data availability can be an obstacle to gene discovery as it is often difficult to decide which genes to pursue further out of a large pool of candidates. Furthermore, the degree of differential expression does not necessarily indicate which genes have the most critical functions in a given pathway because genes that are likely induced as a result of multiple signaling steps in a regulatory cascade often display the highest fold-changes, whereas genes that function earlier in a pathway may show comparatively subtler effects. Additionally, some portion of the genes called as differentially expressed may have had their expression patterns altered as secondary effects from the original treatment and may not, therefore, have any relationship to the process being studied. These drawbacks limit the conclusions that can be drawn about the genes involved in SE and Agrobacterium-mediated transformation from transcriptomic data alone. However, by using other molecular biology techniques to evaluate characteristics of pathways such as protein-protein interactions and protein-DNA binding, as well as using transformation experiments to validate the importance of specific genes, we can compensate for the constraints inherent in transcriptomic analysis in order to develop a more complete picture of the molecular mechanisms underlying these processes. 


\section{Future outlook for enhancing plant regeneration and transformation}

Taken together, the studies that have used differential gene expression to examine the host response to Agrobacterium or elicitor compounds have given us a more complete picture of which genes could be important in Agrobacterium-plant interactions. However, many questions still need to be addressed in order to apply our understanding of the process towards the development or improvement of Agrobacteriummediated transformation techniques for the benefit of crop breeding and basic research (Altpeter et al. 2016). The interaction between Agrobacterium and plants is complex and multi-layered. More focused transcriptomic analyses of specific pathways known to be involved would be desirable to maximize the utility of this approach. Next-generation sequencing technology has allowed greater sensitivity when measuring gene expression and offers attractive novel possibilities for experimental designs, which could give a more refined view of molecular processes. For instance, in order to explore gene regulatory interactions that occur in response to Agrobacterium, RNA-seq could be combined with ChiP-seq to determine the direct induction of genes by key transcription factors.

With respect to biotechnological applications, one could make improvements to the transformation process in various ways informed by differential gene expression data in response to Agrobacterium. For example, an Agrobacterium strain was modified to elicit a weaker plant defense in a study on potato (Vences-Guzmán et al. 2013). In other studies, the composition of tissue culture medium and/or growth conditions was modified in order to minimize plant defense responses (Zhang et al. 2013, Zhang et al. 2016). Because of the similarity of the plant defense response between Agrobacterium 
and other bacterial pathogens, effector proteins originating from other bacteria have even been utilized to repress plant defense gene induction. In one instance, the AvrPto protein from Pseudomonas syringae was conditionally expressed in Arabidopsis plants leading to higher efficiency of Agrobacterium-mediated transformation due to its suppression of plant defenses (Tsuda et al. 2012). As more of the mechanisms involved in Agrobacterium-plant interactions are uncovered, plant transformation researchers will be able to apply some of these discoveries to make new improvements to transformation techniques. 


\section{Reference List}

Alejandri-Ramírez ND, Chávez-Hernández EC, Contreras-Guerra JL, Reyes JL, Dinkova TD. 2018. Small RNA differential expression and regulation in Tuxpeño maize embryogenic callus induction and establishment. Plant Physiology and Biochemistry 122: 78-89.

Alonso JM, Stepanova AN, Leisse TJ, Kim CJ, Chen H, Shinn P, Stevenson DK, Zimmerman J, Barajas P, Cheuk R. 2003. Genome-wide insertional mutagenesis of Arabidopsis thaliana. Science 301: 653-657.

Altpeter F, Springer NM, Bartley LE, Blechl AE, Brutnell TP, Citovsky V, Conrad L, Gelvin SB, Jackson DP, Kausch AP. 2016. Advancing crop transformation in the era of genome editing. The Plant Cell 28: 1510-1520.

Arroyo-Herrera A, Gonzalez AK, Moo RC, Quiroz-Figueroa FR, Loyola-Vargas V, Rodriguez-Zapata L, Suárez-Solís V, Castaño E. 2008. Expression of WUSCHEL in Coffea canephora causes ectopic morphogenesis and increases somatic embryogenesis. Plant Cell, Tissue and Organ Culture 94: 171-180.

Azpiroz-Leehan R, Feldmann KA. 1997. T-DNA insertion mutagenesis in Arabidopsis: going back and forth. Trends in Genetics 13: 152-156.

Barton KA, Brill WJ. 1983. Prospects in plant genetic engineering. Science 219: 671-682.

Beijersbergen A, Smith SJ, Hooykaas PJ. 1994. Localization and topology of VirB proteins of Agrobacterium tumefaciens. Plasmid 32: 212-218.

Beyer P. 2010. Golden Rice and 'Golden'crops for human nutrition. New Biotechnology 27: 478-481.

Bhattacharya A, Sood P, Citovsky V. 2010. The roles of plant phenolics in defence and communication during Agrobacterium and Rhizobium infection. Molecular plant pathology 11: 705-719.

Binns AN, Costantino P. 1998. The Agrobacterium oncogenes. Pages 251-266. The Rhizobiaceae, Springer.

Bouchabke-Coussa O, Obellianne M, Linderme D, Montes E, Maia-Grondard A, Vilaine F, Pannetier C. 2013. Wuschel overexpression promotes somatic embryogenesis and induces organogenesis in cotton (Gossypium hirsutum L.) tissues cultured in vitro. Plant cell reports 32: 675-686. 
Boutilier K, Offringa R, Sharma VK, Kieft H, Ouellet T, Zhang L, Hattori J, Liu C-M, van Lammeren AA, Miki BL. 2002. Ectopic expression of BABY BOOM triggers a conversion from vegetative to embryonic growth. The Plant Cell 14: 1737-1749.

Braybrook SA, Stone SL, Park S, Bui AQ, Le BH, Fischer RL, Goldberg RB, Harada JJ. 2006. Genes directly regulated by $L E A F Y$ COTYLEDON2 provide insight into the control of embryo maturation and somatic embryogenesis. Proceedings of the National Academy of Sciences 103: 3468-3473.

Brisson N, Paszkowski J, Penswick J, Gronenborn B, Potrykus I, Hohn T. 1984. Expression of a bacterial gene in plants by using a viral vector. Nature 310: 511-514.

Casassola A, Brammer SP, Chaves MS, Martinelli JA, Grando MF, Denardin N. 2013. Gene expression: a review on methods for the study of defense-related gene differential expression in plants. Embrapa Trigo-Artigo em periódico indexado (ALICE).

Chen D, Molitor A, Liu C, Shen W-H. 2010. The Arabidopsis PRC1-like ring-finger proteins are necessary for repression of embryonic traits during vegetative growth. Cell research 20: 1332-1344.

Chen K, Wang Y, Zhang R, Zhang H, Gao C. 2019. CRISPR/Cas genome editing and precision plant breeding in agriculture. Annual review of plant biology 70: 667697.

Cheng M, Lowe BA, Spencer TM, Ye X, Armstrong CL. 2004. Factors influencing Agrobacterium-mediated transformation of monocotyledonous species. In Vitro Cellular \& Developmental Biology-Plant 40: 31-45.

Chhun T, Chong SY, Park BS, Wong ECC, Yin J-L, Kim M, Chua N-H. 2016. HSI2 repressor recruits MED13 and HDA6 to down-regulate seed maturation gene expression directly during Arabidopsis early seedling growth. Plant and Cell Physiology 57: 1689-1706.

Chilton M-D, Drummond MH, Merlo DJ, Sciaky D, Montoya AL, Gordon MP, Nester EW. 1977. Stable incorporation of plasmid DNA into higher plant cells: the molecular basis of crown gall tumorigenesis. Cell 11: 263-271.

Christian M, Cermak T, Doyle EL, Schmidt C, Zhang F, Hummel A, Bogdanove AJ, Voytas DF. 2010. Targeting DNA double-strand breaks with TAL effector nucleases. Genetics 186: 757-761. 
Christie PJ, Vogel JP. 2000. Bacterial type IV secretion: conjugation systems adapted to deliver effector molecules to host cells. Trends in microbiology 8: 354-360.

Cong L, Ran FA, Cox D, Lin S, Barretto R, Habib N, Hsu PD, Wu X, Jiang W, Marraffini LA. 2013. Multiplex genome engineering using CRISPR/Cas systems. Science 339: 819-823.

Cui H, Xiang T, Zhou JM. 2009. Plant immunity: a lesson from pathogenic bacterial effector proteins. Cellular microbiology 11: 1453-1461.

D’Halluin K, Vanderstraeten C, Stals E, Cornelissen M, Ruiter R. 2008. Homologous recombination: a basis for targeted genome optimization in crop species such as maize. Plant biotechnology journal 6: 93-102.

De Block M, Herrera-Estrella L, Van Montagu M, Schell J, Zambryski P. 1984. Expression of foreign genes in regenerated plants and in their progeny. The EMBO Journal 3: 1681-1689.

De Cleene M. 1985. The susceptibility of monocotyledons to Agrobacterium tumefaciens. Journal of Phytopathology 113: 81-89.

De Cleene M, De Ley J. 1976. The host range of crown gall. The botanical review 42: 389466.

Deng W, Luo K, Li Z, Yang Y. 2009. A novel method for induction of plant regeneration via somatic embryogenesis. Plant Science 177: 43-48.

Ditt RF, Nester EW, Comai L. 2001. Plant gene expression response to Agrobacterium tumefaciens. Proceedings of the National Academy of Sciences 98: 10954-10959.

Ditt RF, Nester E, Comai L. 2005. The plant cell defense and Agrobacterium tumefaciens. FEMS microbiology letters 247: 207-213.

Ditt RF, Kerr KF, de Figueiredo P, Delrow J, Comai L, Nester EW. 2006. The Arabidopsis thaliana transcriptome in response to Agrobacterium tumefaciens. Molecular plant-microbe interactions 19: 665-681.

Djamei A, Pitzschke A, Nakagami H, Rajh I, Hirt H. 2007. Trojan horse strategy in Agrobacterium transformation: abusing MAPK defense signaling. Science 318 : 453-456.

Dodeman VL, Ducreux G, Kreis M. 1997. Zygotic embryogenesis versus somatic embryogenesis. Journal of Experimental Botany 48: 1493-1509. 
Duan K, Willig CJ, De Tar JR, Spollen WG, Zhang ZJ. 2018. Transcriptomic analysis of Arabidopsis seedlings in response to an Agrobacterium-mediated transformation process. Molecular plant-microbe interactions 31: 445-459.

Dudits D, Györgyey J, Bögre L, Bakó L. 1995. Molecular biology of somatic embryogenesis. Pages 267-308. In vitro embryogenesis in plants, Springer.

Elhiti M, Stasolla C, Wang A. 2013. Molecular regulation of plant somatic embryogenesis. In Vitro Cellular \& Developmental Biology-Plant 49: 631-642.

Escobar MA, Dandekar AM. 2003. Agrobacterium tumefaciens as an agent of disease. Trends in plant science 8: 380-386.

Evenson RE, Gollin D. 2003. Assessing the impact of the Green Revolution, 1960 to 2000. science 300: 758-762.

Fauser F, Roth N, Pacher M, Ilg G, Sánchez-Fernández R, Biesgen C, Puchta H. 2012. In planta gene targeting. Proceedings of the National Academy of Sciences 109: 7535-7540.

Fehér A. 2015. Somatic embryogenesis-stress-induced remodeling of plant cell fate. Biochimica et Biophysica Acta (BBA)-Gene Regulatory Mechanisms 1849: 385402.

Fehér A. 2019. Callus, dedifferentiation, totipotency, somatic embryogenesis: what these terms mean in the era of molecular plant biology? Frontiers in plant science 10: 536.

Filonova LH, Bozhkov PV, Arnold Sv. 2000. Developmental pathway of somatic embryogenesis in Picea abies as revealed by time-lapse tracking. Journal of Experimental Botany 51: 249-264.

Fisher RA. 1936. Has Mendel's work been rediscovered? Annals of Science 1: 115-137.

Florez SL, Erwin RL, Maximova SN, Guiltinan MJ, Curtis WR. 2015. Enhanced somatic embryogenesis in Theobroma cacao using the homologous BABY BOOM transcription factor. BMC plant biology 15: 1-13.

Fromm ME, Taylor LP, Walbot V. 1986. Stable transformation of maize after gene transfer by electroporation. Nature 319: 791-793. 
Gaj MD. 2004. Factors influencing somatic embryogenesis induction and plant regeneration with particular reference to Arabidopsis thaliana (L.) Heynh. Plant Growth Regulation 43: 27-47.

Gaj MD, Zhang S, Harada JJ, Lemaux PG. 2005. Leafy cotyledon genes are essential for induction of somatic embryogenesis of Arabidopsis. Planta 222: 977-988.

Gallois J-L, Nora FR, Mizukami Y, Sablowski R. 2004. WUSCHEL induces shoot stem cell activity and developmental plasticity in the root meristem. Genes \& development 18: 375-380.

Gamborg O, Murashige T, Thorpe T, Vasil I. 1976. Plant tissue culture media. In vitro 12: 473-478.

Garcês HM, Koenig D, Townsley BT, Kim M, Sinha NR. 2014. Truncation of LEAFY COTYLEDON1 protein is required for asexual reproduction in Kalanchoë daigremontiana. Plant Physiology 165: 196-206.

García-Rodríguez FM, Schrammeijer B, Hooykaas PJ. 2006. The Agrobacterium VirE3 effector protein: a potential plant transcriptional activator. Nucleic acids research 34: 6496-6504.

Gelvin SB. 2000. Agrobacterium and plant genes involved in T-DNA transfer and integration. Annual review of plant biology 51: 223-256.

Gelvin SB. 2010. Plant proteins involved in Agrobacterium-mediated genetic transformation. Annual review of phytopathology 48: 45-68.

Gelvin SB. 2017. Integration of Agrobacterium T-DNA into the plant genome. Annual review of genetics 51: 195-217.

Gliwicka M, Nowak K, Balazadeh S, Mueller-Roeber B, Gaj MD. 2013. Extensive modulation of the transcription factor transcriptome during somatic embryogenesis in Arabidopsis thaliana. Plos one 8: e69261.

Gordon-Kamm B, Sardesai N, Arling M, Lowe K, Hoerster G, Betts S, Jones T. 2019. Using morphogenic genes to improve recovery and regeneration of transgenic plants. Plants 8: 38.

Grassini P, Eskridge KM, Cassman KG. 2013. Distinguishing between yield advances and yield plateaus in historical crop production trends. Nature communications 4: 111. 
Grosser JW, Ollitrault P, Olivares-Fuster O. 2000. Somatic hybridization in citrus: an effective tool to facilitate variety improvement. In Vitro Cellular \& Developmental Biology-Plant 36: 434-449.

Guan Y, Li S-G, Fan X-F, Su Z-H. 2016. Application of somatic embryogenesis in woody plants. Frontiers in plant science 7: 938.

Guo F, Liu C, Xia H, Bi Y, Zhao C, Zhao S, Hou L, Li F, Wang X. 2013. Induced expression of AtLEC1 and AtLEC2 differentially promotes somatic embryogenesis in transgenic tobacco plants. PLoS One 8: e71714.

Haecker A, Groß-Hardt R, Geiges B, Sarkar A, Breuninger H, Herrmann M, Laux T. 2004. Expression dynamics of WOX genes mark cell fate decisions during early embryonic patterning in Arabidopsis thaliana. Development 131: 657-668.

Harding EW, Tang W, Nichols KW, Fernandez DE, Perry SE. 2003. Expression and maintenance of embryogenic potential is enhanced through constitutive expression of AGAMOUS-Like 15. Plant Physiology 133: 653-663.

Hecht V, Vielle-Calzada J-P, Hartog MV, Schmidt ED, Boutilier K, Grossniklaus U, de Vries SC. 2001. The Arabidopsis SOMATIC EMBRYOGENESIS RECEPTOR KINASE 1 gene is expressed in developing ovules and embryos and enhances embryogenic competence in culture. Plant Physiology 127: 803-816.

Heidmann I, De Lange B, Lambalk J, Angenent GC, Boutilier K. 2011. Efficient sweet pepper transformation mediated by the $B A B Y B O O M$ transcription factor. Plant cell reports 30: 1107-1115.

Holme IB, Gregersen PL, Brinch-Pedersen H. 2019. Induced genetic variation in crop plants by random or targeted mutagenesis: convergence and differences. Frontiers in plant science 10: 1468.

Horstman A, Bemer M, Boutilier K. 2017a. A transcriptional view on somatic embryogenesis. Regeneration 4: 201-216.

Horstman A, Li M, Heidmann I, Weemen M, Chen B, Muino JM, Angenent GC, Boutilier K. 2017b. The BABY BOOM transcription factor activates the LEC1-ABI3-FUS3LEC2 network to induce somatic embryogenesis. Plant physiology $175: 848-857$.

Horstman A, Fukuoka H, Muino JM, Nitsch L, Guo C, Passarinho P, Sanchez-Perez G, Immink R, Angenent G, Boutilier K. 2015. AIL and HDG proteins act antagonistically to control cell proliferation. Development 142: 454-464. 
Huang S, Hill RD, Wally OS, Dionisio G, Ayele BT, Jami SK, Stasolla C. 2014. Hemoglobin control of cell survival/death decision regulates in vitro plant embryogenesis. Plant physiology 165: 810-825.

Huang T-K, Puchta H. 2019. CRISPR/Cas-mediated gene targeting in plants: finally a turn for the better for homologous recombination. Plant cell reports 38: 443-453.

Ikeuchi M, Iwase A, Rymen B, Harashima H, Shibata M, Ohnuma M, Breuer C, Morao AK, de Lucas M, De Veylder L. 2015. PRC2 represses dedifferentiation of mature somatic cells in Arabidopsis. Nature Plants 1: 1-7.

Jia H, Suzuki M, McCarty DR. 2014. Regulation of the seed to seedling developmental phase transition by the LAFL and VAL transcription factor networks. Wiley Interdisciplinary Reviews: Developmental Biology 3: 135-145.

Jiménez VM. 2005. Involvement of plant hormones and plant growth regulators on in vitro somatic embryogenesis. Plant Growth Regulation 47: 91-110.

Jinek M, Chylinski K, Fonfara I, Hauer M, Doudna JA, Charpentier E. 2012. A programmable dual-RNA-guided DNA endonuclease in adaptive bacterial immunity. science 337: 816-821.

Johri B, Bhojwani S. 1965. Growth responses of mature endosperm in cultures. Nature 208: $1345-1347$.

Kleter GA, Peijnenburg AA, Aarts HJ. 2005. Health considerations regarding horizontal transfer of microbial transgenes present in genetically modified crops. Journal of Biomedicine and Biotechnology 2005: 326-352.

Klimaszewska K, Pelletier G, Overton C, Stewart D, Rutledge RG. 2010. Hormonally regulated overexpression of Arabidopsis WUS and conifer LEC1 (CHAP3A) in transgenic white spruce: implications for somatic embryo development and somatic seedling growth. Plant cell reports 29: 723-734.

Kojima M, Sparthana P, Teixeira da Silva J, Nogawa M. 2006. Development of in planta transformation methods using Agrobacterium tumefaciens. Floriculture, Ornamental and Plant Biotechnology: Advances and Topical: 41-48.

Kuldau GA, De Vos G, Owen J, McCaffrey G, Zambryski P. 1990. The virB operon of Agrobacterium tumefaciens pTiC58 encodes 11 open reading frames. Molecular and General Genetics MGG 221: 256-266. 
Kumar V, Jain M. 2015. The CRISPR-Cas system for plant genome editing: advances and opportunities. Journal of experimental botany 66: 47-57.

Kyndt T, Quispe D, Zhai H, Jarret R, Ghislain M, Liu Q, Gheysen G, Kreuze JF. 2015. The genome of cultivated sweet potato contains Agrobacterium T-DNAs with expressed genes: an example of a naturally transgenic food crop. Proceedings of the National Academy of Sciences 112: 5844-5849.

Lacroix B, Citovsky V. 2013. The roles of bacterial and host plant factors in Agrobacterium-mediated genetic transformation. International Journal of Developmental Biology 57: 467-481.

Lacroix B, Vaidya M, Tzfira T, Citovsky V. 2005. The VirE3 protein of Agrobacterium mimics a host cell function required for plant genetic transformation. The EMBO Journal 24: 428-437.

Laibach F. 1929. Ectogenesis in plants: methods and genetic possibilities of propagating embryos otherwise dying in the seed. Journal of Heredity 20: 201-208.

Lapham R, Lee L-Y, Tsugama D, Lee S, Mengiste T, Gelvin SB. 2018. VIP1 and its homologs are not required for Agrobacterium-mediated transformation, but play a role in Botrytis and salt stress responses. Frontiers in plant science 9: 749.

Laux T, Mayer K, Berger J, Jurgens G. 1996. The WUSCHEL gene is required for shoot and floral meristem integrity in Arabidopsis. Development 122: 87-96.

Lawrence W, Davies D. 1985. A method for the microinjection and culture of protoplasts at very low densities. Plant cell reports 4: 33-35.

Lee C-W, Efetova M, Engelmann JC, Kramell R, Wasternack C, Ludwig-Müller J, Hedrich R, Deeken R. 2009. Agrobacterium tumefaciens promotes tumor induction by modulating pathogen defense in Arabidopsis thaliana. The Plant Cell 21: 29482962.

Leibfried A, To JP, Busch W, Stehling S, Kehle A, Demar M, Kieber JJ, Lohmann JU. 2005. WUSCHEL controls meristem function by direct regulation of cytokinin-inducible response regulators. Nature 438: 1172-1175.

Lin Q, Zong Y, Xue C, Wang S, Jin S, Zhu Z, Wang Y, Anzalone AV, Raguram A, Doman JL. 2020. Prime genome editing in rice and wheat. Nature biotechnology 38: 582585. 
Lin Y, Lin L, Lai R, Liu W, Chen Y, Zhang Z, XuHan X, Lai Z. 2015. MicroRNA390-directed TAS3 cleavage leads to the production of tasiRNA-ARF3/4 during somatic embryogenesis in Dimocarpus longan Lour. Frontiers in plant science 6: 1119.

Liu J, Xu X, Deng X. 2005. Intergeneric somatic hybridization and its application to crop genetic improvement. Plant Cell, Tissue and Organ Culture 82: 19-44.

Liu J, Deng S, Wang H, Ye J, Wu H-W, Sun H-X, Chua N-H. 2016. CURLY LEAF regulates gene sets coordinating seed size and lipid biosynthesis. Plant physiology 171 : 424-436.

Loo S-W. 1945. Cultivation of excised stem tips of asparagus in vitro. American Journal of Botany: 13-17.

Lotan T, Ohto M-a, Yee KM, West MA, Lo R, Kwong RW, Yamagishi K, Fischer RL, Goldberg RB, Harada JJ. 1998. Arabidopsis LEAFY COTYLEDON1 is sufficient to induce embryo development in vegetative cells. Cell 93: 1195-1205.

Lowe K, Wu E, Wang N, Hoerster G, Hastings C, Cho M-J, Scelonge C, Lenderts B, Chamberlin M, Cushatt J. 2016. Morphogenic regulators Baby boom and Wuschel improve monocot transformation. The Plant Cell 28: 1998-2015.

Loyola-Vargas VM. 2016. The history of somatic embryogenesis. Pages 11-22. Somatic embryogenesis: fundamental aspects and applications, Springer.

Lup SD, Tian X, Xu J, Pérez-Pérez JM. 2016. Wound signaling of regenerative cell reprogramming. Plant Science 250: 178-187.

Magori S, Citovsky V. 2011. Agrobacterium counteracts host-induced degradation of its effector F-box protein. Science signaling 4: ra69-ra69.

Maher MF, Nasti RA, Vollbrecht M, Starker CG, Clark MD, Voytas DF. 2020. Plant gene editing through de novo induction of meristems. Nature biotechnology 38: 8489.

Malamy JE, Benfey PN. 1997. Organization and cell differentiation in lateral roots of Arabidopsis thaliana. Development 124: 33-44.

Mathieu M, Lelu-Walter M-A, Blervacq A-S, David H, Hawkins S, Neutelings G. 2006. Germin-like genes are expressed during somatic embryogenesis and early development of conifers. Plant molecular biology 61: 615-627. 
Matveeva TV, Otten L. 2019. Widespread occurrence of natural genetic transformation of plants by Agrobacterium. Plant Molecular Biology 101: 415-437.

Mayavan S, Subramanyam K, Arun M, Rajesh M, Dev GK, Sivanandhan G, Jaganath B, Manickavasagam M, Selvaraj N, Ganapathi A. 2013. Agrobacterium tumefaciensmediated in planta seed transformation strategy in sugarcane. Plant cell reports 32: 1557-1574.

Miroshnichenko D, Shulga O, Timerbaev V, Dolgov S. 2019. Generation of nontransgenic genome-edited plants: achievements, challenges and prospects. Biotechnology 35: 3-26.

Mittler R, Blumwald E. 2010. Genetic engineering for modern agriculture: challenges and perspectives. Annual review of plant biology 61: 443-462.

Morris RO. 1986. Genes specifying auxin and cytokinin biosynthesis in phytopathogens. Annual Review of Plant Physiology 37: 509-538.

Muir W, Hildebrandt A, Riker A. 1954. Plant tissue cultures produced from single isolated cells. Science 119: 877-878.

Muir W, Hildebrandt A, Riker A. 1958. The preparation, isolation, and growth in culture of single cells from higher plants. American Journal of Botany: 589-597.

Murashige T, Skoog F. 1962. A revised medium for rapid growth and bio assays with tobacco tissue cultures. Physiologia plantarum 15: 473-497.

Murovec J, Bohanec B. 2011. Haploids and doubled haploids in plant breeding. Plant Breeding, Dr. Ibrokhim Abdurakhmonov (Ed.).-2012.-P: 87-106.

Mysore KS, Bassuner B, Deng X-b, Darbinian NS, Motchoulski A, Ream W, Gelvin SB. 1998. Role of the Agrobacterium tumefaciens VirD2 protein in T-DNA transfer and integration. Molecular plant-microbe interactions 11: 668-683.

Namasivayam P. 2007. Acquisition of embryogenic competence during somatic embryogenesis. Plant Cell, Tissue and Organ Culture 90: 1-8.

Niazian M, Noori SS, Galuszka P, Mortazavian SMM. 2017. Tissue culture-based Agrobacterium-mediated and in planta transformation methods. Soil and Water Research 53: 133-143. 
Niu X, Zhou M, Henkel CV, van Heusden GPH, Hooykaas PJ. 2015. The Agrobacterium tumefaciens virulence protein VirE3 is a transcriptional activator of the F-box gene VBF. The Plant Journal 84: 914-924.

Panattoni A, Luvisi A, Triolo E. 2013. Elimination of viruses in plants: twenty years of progress. Spanish Journal of Agricultural Research: 173-188.

Parcy F, Valon C, Kohara A, Miséra S, Giraudat J. 1997. The ABSCISIC ACID-INSENSITIVE3, FUSCA3, and LEAFY COTYLEDON1 loci act in concert to control multiple aspects of Arabidopsis seed development. The Plant Cell 9: 1265-1277.

Pasternak TP, Prinsen E, Ayaydin F, Miskolczi P, Potters G, Asard H, Van Onckelen HA, Dudits D, Fehér A. 2002. The role of auxin, $p H$, and stress in the activation of embryogenic cell division in leaf protoplast-derived cells of alfalfa. Plant physiology 129: 1807-1819.

Peterson GE. 1967. The discovery and development of 2, 4-D. Agricultural History 41: 243-254.

Phillips GC, Garda M. 2019. Plant tissue culture media and practices: an overview. In Vitro Cellular \& Developmental Biology-Plant 55: 242-257.

Pu X-A, Goodman R. 1993. Attachment of agrobacteria to grape cells. Applied and environmental microbiology 59: 2572-2577.

Puchta H. 2002. Gene replacement by homologous recombination in plants. Functional Genomics: 173-182.

Qi Y, Zhang Y, Zhang F, Baller JA, Cleland SC, Ryu Y, Starker CG, Voytas DF. 2013. Increasing frequencies of site-specific mutagenesis and gene targeting in Arabidopsis by manipulating DNA repair pathways. Genome research 23: 547554.

Quak F. 1961. Heat treatment and substances inhibiting virus multiplication in meristem culture to obtain virus-free plants. Adv. Hort. Sci. Appl 1: 144-148.

Quiroz-Figueroa F, Fuentes-Cerda C, Rojas-Herrera R, Loyola-Vargas V. 2002. Histological studies on the developmental stages and differentiation of two different somatic embryogenesis systems of Coffea arabica. Plant Cell Reports 20: 1141-1149.

Quiroz-Figueroa FR, Rojas-Herrera R, Galaz-Avalos RM, Loyola-Vargas VM. 2006. Embryo production through somatic embryogenesis can be used to study cell differentiation in plants. Plant Cell, Tissue and Organ Culture 86: 285-301. 
Reddy MC, Murthy KSR, Pullaiah T. 2012. Synthetic seeds: A review in agriculture and forestry. African Journal of Biotechnology 11: 14254-14275.

Reinert J. 1958. Untersuchungen uber die Morphogenese an Gewebekulturen. Ber Dtsch Bot Ges 71: 15.

Sabana AA, Rajesh MK, Antony G. 2020. Dynamic changes in the expression pattern of miRNAs and associated target genes during coconut somatic embryogenesis. Planta 251: 1-18.

Sanford JC, Klein TM, Wolf ED, Allen N. 1987. Delivery of substances into cells and tissues using a particle bombardment process. Particulate Science and Technology 5: 27-37.

Schiml S, Fauser F, Puchta H. 2014. The CRISPR/Cas system can be used as nuclease for in planta gene targeting and as paired nickases for directed mutagenesis in Arabidopsis resulting in heritable progeny. The Plant Journal 80: 1139-1150.

Schrammeijer B, Dulk-Ras Ad, Vergunst AC, Jurado Jácome E, Hooykaas PJ. 2003. Analysis of Vir protein translocation from Agrobacterium tumefaciens using Saccharomyces cerevisiae as a model: evidence for transport of a novel effector protein VirE3. Nucleic Acids Research 31: 860-868.

Shi Y, Lee LY, Gelvin SB. 2014. Is VIP1 important for Agrobacterium-mediated transformation? The Plant Journal 79: 848-860.

Shirasu K, Morel P, Kado C. 1990. Characterization of the virB operon of an Agrobacterium tumefaciens Ti plasmid: nucleotide sequence and protein analysis. Molecular microbiology 4: 1153-1163.

Skoog F, Miller C. 1957. Chemical regulation of growth and organ formation in plant tissues cultured. Vitro Symp Soc Exp Biol.

Solís-Ramos L, González-Estrada T, Nahuath-Dzib S, Zapata-Rodriguez L, Castaño E. 2009. Overexpression of WUSCHEL in C. chinense causes ectopic morphogenesis. Plant Cell, Tissue and Organ Culture (PCTOC) 96: 279-287.

Solís-Ramos LY, Andrade-Torres A, Salín CMO, de la Serna EC, Carbonell LAS. 2012. Somatic embryogenesis in recalcitrant plants: INTECH Open Access Publisher.

Somssich M. 2019. A short history of plant transformation. PeerJ Preprints 7: e27556v27552. 
Srinivasan C, Liu Z, Heidmann I, Supena EDJ, Fukuoka H, Joosen R, Lambalk J, Angenent G, Scorza R, Custers JB. 2007. Heterologous expression of the BABY BOOM AP2/ERF transcription factor enhances the regeneration capacity of tobacco (Nicotiana tabacum L.). Planta 225: 341-351.

Staskawicz BJ, Ausubel FM, Baker BJ, Ellis JG, Jones J. 1995. Molecular genetics of plant disease resistance. Science 268: 661-667.

Steward F, Mapes MO, Mears K. 1958. Growth and organized development of cultured cells. II. Organization in cultures grown from freely suspended cell. American Journal of Botany 45: 705-708.

Stone SL, Kwong LW, Yee KM, Pelletier J, Lepiniec L, Fischer RL, Goldberg RB, Harada JJ. 2001. LEAFY COTYLEDON2 encodes a B3 domain transcription factor that induces embryo development. Proceedings of the National Academy of Sciences 98 : 11806-11811.

Stone SL, Braybrook SA, Paula SL, Kwong LW, Meuser J, Pelletier J, Hsieh T-F, Fischer RL, Goldberg RB, Harada JJ. 2008. Arabidopsis LEAFY COTYLEDON2 induces maturation traits and auxin activity: implications for somatic embryogenesis. Proceedings of the National Academy of Sciences 105: 3151-3156.

Su YH, Zhao XY, Liu YB, Zhang CL, O'Neill SD, Zhang XS. 2009. Auxin-induced WUS expression is essential for embryonic stem cell renewal during somatic embryogenesis in Arabidopsis. The Plant Journal 59: 448-460.

Sugimoto K, Jiao Y, Meyerowitz EM. 2010. Arabidopsis regeneration from multiple tissues occurs via a root development pathway. Developmental cell 18: 463-471.

Sugimoto K, Gordon SP, Meyerowitz EM. 2011. Regeneration in plants and animals: dedifferentiation, transdifferentiation, or just differentiation? Trends in cell biology 21: 212-218.

Swamy NR. 1958. Culture of nucellar tissue of Citrus in vitro. Experientia 14: 111-112.

Szyrajew K, Bielewicz D, Dolata J, Wójcik AM, Nowak K, Szczygieł-Sommer A, Szweykowska-Kulinska Z, Jarmolowski A, Gaj MD. 2017. MicroRNAs are intensively regulated during induction of somatic embryogenesis in Arabidopsis. Frontiers in plant science 8: 18.

Tao Y, Xie Z, Chen W, Glazebrook J, Chang H-S, Han B, Zhu T, Zou G, Katagiri F. 2003. Quantitative nature of Arabidopsis responses during compatible and 
incompatible interactions with the bacterial pathogen Pseudomonas syringae. The Plant Cell 15: 317-330.

Thakare D, Tang W, Hill K, Perry SE. 2008. The MADS-domain transcriptional regulator AGAMOUS-LIKE15 promotes somatic embryo development in Arabidopsis and soybean. Plant physiology 146: 1663-1672.

Thompson DV, Melchers LS, Idler KB, Schilperoort RA, Hooykaas PJ. 1988. Analysis of the complete nucleotide sequence of the Agrobacterium tumefaciens virB operon. Nucleic acids research 16: 4621-4636.

Thorpe TA. 2007. History of plant tissue culture. Molecular biotechnology 37: 169-180.

Tie W, Zhou F, Wang L, Xie W, Chen H, Li X, Lin Y. 2012. Reasons for lower transformation efficiency in indica rice using Agrobacterium tumefaciensmediated transformation: lessons from transformation assays and genome-wide expression profiling. Plant molecular biology 78: 1-18.

Tisserat B, Esan E, Murashige T. 1979. Somatic embryogenesis in angiosperms. Horticultural reviews 1: 1-78.

Tomlinson AD, Fuqua C. 2009. Mechanisms and regulation of polar surface attachment in Agrobacterium tumefaciens. Current opinion in microbiology 12: 708-714.

Toonen MA, Hendriks T, Schmidt ED, Verhoeven HA, van Kammen A, de Vries SC. 1994. Description of somatic-embryo-forming single cells in carrot suspension cultures employing video cell tracking. Planta 194: 565-572.

Townsend JA, Wright DA, Winfrey RJ, Fu F, Maeder ML, Joung JK, Voytas DF. 2009. Highfrequency modification of plant genes using engineered zinc-finger nucleases. Nature 459: 442-445.

Tsuda K, Qi Y, Nguyen LV, Bethke G, Tsuda Y, Glazebrook J, Katagiri F. 2012. An efficient Agrobacterium-mediated transient transformation of Arabidopsis. The Plant Journal 69: 713-719.

Tsuwamoto R, Yokoi S, Takahata Y. 2010. Arabidopsis EMBRYOMAKER encoding an AP2 domain transcription factor plays a key role in developmental change from vegetative to embryonic phase. Plant molecular biology 73: 481-492.

Tvorogova VE, Fedorova YA, Potsenkovskaya EA, Kudriashov AA, Efremova EP, Kvitkovskaya VA, Wolabu TW, Zhang F, Tadege M, Lutova LA. 2019. The WUSCHEL-related homeobox transcription factor MtWOX9-1 stimulates somatic 
embryogenesis in Medicago truncatula. Plant Cell, Tissue and Organ Culture (PCTOC) 138: 517-527.

Tzfira T, Citovsky V. 2006. Agrobacterium-mediated genetic transformation of plants: biology and biotechnology. Current opinion in biotechnology 17: 147-154.

Tzfira T, Vaidya M, Citovsky V. 2001. VIP1, an Arabidopsis protein that interacts with Agrobacterium VirE2, is involved in VirE2 nuclear import and Agrobacterium infectivity. The EMBO Journal 20: 3596-3607.

Tzfira T, Vaidya M, Citovsky V. 2004. Involvement of targeted proteolysis in plant genetic transformation by Agrobacterium. Nature 431: 87-92.

Vasil V, Hildebrandt A. 1965. Differentiation of tobacco plants from single, isolated cells in microcultures. Science 150: 889-892.

Veena, Jiang H, Doerge R, Gelvin SB. 2003. Transfer of T-DNA and Vir proteins to plant cells by Agrobacterium tumefaciens induces expression of host genes involved in mediating transformation and suppresses host defense gene expression. The Plant Journal 35: 219-236.

Vences-Guzmán MÁ, Guan Z, Bermúdez-Barrientos JR, Geiger O, Sohlenkamp C. 2013. Agrobacteria lacking ornithine lipids induce more rapid tumour formation. Environmental microbiology 15: 895-906.

Vergunst AC, Schrammeijer B, den Dulk-Ras A, de Vlaam CM, Regensburg-Tuınk TJ, Hooykaas PJ. 2000. VirB/D4-dependent protein translocation from Agrobacterium into plant cells. Science 290: 979-982.

Von Arnold S, Sabala I, Bozhkov P, Dyachok J, Filonova L. 2002. Developmental pathways of somatic embryogenesis. Plant cell, Tissue and Organ culture 69: 233-249.

Wang L, Chong K. 2016. The essential role of cytokinin signaling in root apical meristem formation during somatic embryogenesis. Frontiers in plant science 6: 1196.

Wang Y, Peng W, Zhou X, Huang F, Shao L, Luo M. 2014. The putative Agrobacterium transcriptional activator-like virulence protein VirD5 may target T-complex to prevent the degradation of coat proteins in the plant cell nucleus. New Phytologist 203: 1266-1281.

Ward DV, Draper O, Zupan JR, Zambryski PC. 2002. Peptide linkage mapping of the Agrobacterium tumefaciens vir-encoded type IV secretion system reveals protein 
subassemblies. Proceedings of the National Academy of Sciences 99: 1149311500.

Ward J, Akiyoshi D, Regier D, Datta A, Gordon M, Nester E. 1988. Characterization of the virB operon from an Agrobacterium tumefaciens Ti plasmid. Journal of Biological Chemistry 263: 5804-5814.

Watson B, Currier TC, Gordon MP, Chilton M, Nester E. 1975. Plasmid required for virulence of Agrobacterium tumefaciens. Journal of Bacteriology 123: 255-264.

White PR. 1934. Potentially unlimited growth of excised tomato root tips in a liquid medium. Plant Physiology 9: 585.

White PR. 1939a. Controlled differentiation in a plant tissue culture. Bulletin of the Torrey Botanical Club: 507-513.

White PR. 1939b. Potentially unlimited growth of excised plant callus in an artificial nutrient. American Journal of Botany 26: 59-64.

Williams E, Maheswaran G. 1986. Somatic embryogenesis: factors influencing coordinated behaviour of cells as an embryogenic group. Annals of Botany 57: 443-462.

Wójcik AM, Nodine MD, Gaj MD. 2017. miR160 and miR166/165 contribute to the LEC2mediated auxin response involved in the somatic embryogenesis induction in Arabidopsis. Frontiers in plant science 8: 2024.

Wolter F, Puchta H. 2019. In planta gene targeting can be enhanced by the use of CRISPR/Cas12a. The Plant Journal 100: 1083-1094.

Wood DW, Setubal JC, Kaul R, Monks DE, Kitajima JP, Okura VK, Zhou Y, Chen L, Wood $\mathrm{GE}$, Almeida NF. 2001. The genome of the natural genetic engineer Agrobacterium tumefaciens C58. science 294: 2317-2323.

Xu L, Najeeb U, Tang G, Gu H, Zhang G, He Y, Zhou W. 2007. Haploid and doubled haploid technology. Advances in botanical research 45: 181-216.

Yang C, Bratzel F, Hohmann N, Koch M, Turck F, Calonje M. 2013. VAL-and AtBMI1mediated $\mathrm{H} 2 \mathrm{Aub}$ initiate the switch from embryonic to postgerminative growth in Arabidopsis. Current Biology 23: 1324-1329.

Yang X, Zhang X. 2010. Regulation of somatic embryogenesis in higher plants. Critical Reviews in Plant Science 29: 36-57. 
Yang Z, Li C, Wang Y, Zhang C, Wu Z, Zhang X, Liu C, Li F. 2014. GhAGL15s, preferentially expressed during somatic embryogenesis, promote embryogenic callus formation in cotton (Gossypium hirsutum L.). Molecular Genetics and Genomics 289: 873-883.

Zaltsman A, Krichevsky A, Loyter A, Citovsky V. 2010. Agrobacterium induces expression of a host F-box protein required for tumorigenicity. Cell host \& microbe 7: 197209.

Zhang H, Rider Jr SD, Henderson JT, Fountain M, Chuang K, Kandachar V, Simons A, Edenberg HJ, Romero-Severson J, Muir WM. 2008. The CHD3 remodeler PICKLE promotes trimethylation of histone $\mathrm{H} 3$ lysine 27. Journal of Biological Chemistry 283: 22637-22648.

Zhang W-J, Dewey RE, Boss W, Phillippy BQ, Qu R. 2013. Enhanced Agrobacteriummediated transformation efficiencies in monocot cells is associated with attenuated defense responses. Plant molecular biology 81: 273-286.

Zhang X, van Heusden GPH, Hooykaas PJ. 2017. Virulence protein VirD5 of Agrobacterium tumefaciens binds to kinetochores in host cells via an interaction with Spt4. Proceedings of the National Academy of Sciences 114: 10238-10243.

Zhang Y-M, Liu Z-H, Yang R-J, Li G-L, Guo X-L, Zhang H-N, Zhang H-M, Di R, Zhao Q-S, Zhang M-C. 2016. Improvement of soybean transformation via Agrobacterium tumefaciens methods involving $\alpha$-aminooxyacetic acid and sonication treatments enlightened by gene expression profile analysis. Plant cell reports 35: 1259-1271.

Zhao P, Begcy K, Dresselhaus T, Sun M-X. 2017. Does early embryogenesis in eudicots and monocots involve the same mechanism and molecular players? Plant Physiology 173: 130-142.

Zheng Y, Ren N, Wang H, Stromberg AJ, Perry SE. 2009. Global identification of targets of the Arabidopsis MADS domain protein AGAMOUS-Like15. The Plant Cell 21: 2563-2577.

Zhou T, Yang X, Guo K, Deng J, Xu J, Gao W, Lindsey K, Zhang X. 2016. ROS homeostasis regulates somatic embryogenesis via the regulation of auxin signaling in cotton. Molecular \& Cellular Proteomics 15: 2108-2124.

Zhou X, Wang K, Lv D, Wu C, Li J, Zhao P, Lin Z, Du L, Yan Y, Ye X. 2013a. Global Analysis of Differentially Expressed Genes and Proteins in the Wheat Callus Infected by Agrobacterium tumefaciens. PloS one 8: e79390. 
Zhou Y, Tan B, Luo M, Li Y, Liu C, Chen C, Yu C-W, Yang S, Dong S, Ruan J. 2013b. HISTONE DEACETYLASE19 interacts with HSL1 and participates in the repression of seed maturation genes in Arabidopsis seedlings. The Plant Cell 25: 134-148.

Ziemienowicz A, Merkle T, Schoumacher F, Hohn B, Rossi L. 2001. Import of Agrobacterium T-DNA into plant nuclei: two distinct functions of VirD2 and VirE2 proteins. The Plant Cell 13: 369-383.

Zipfel C, Robatzek S. 2010. Pathogen-associated molecular pattern-triggered immunity: veni, vidi...? Plant physiology 154: 551-554.

Zipfel C, Kunze G, Chinchilla D, Caniard A, Jones JD, Boller T, Felix G. 2006. Perception of the bacterial PAMP EF-Tu by the receptor EFR restricts Agrobacterium-mediated transformation. Cell 125: 749-760.

Zuo J, Niu QW, Frugis G, Chua NH. 2002. The WUSCHEL gene promotes vegetative-toembryonic transition in Arabidopsis. The Plant Journal 30: 349-359. 


\section{Chapter 2}

Using transcriptional dynamics to uncover genes expressed during somatic embryogenesis in Sorghum bicolor

Note: Phat Tien Do assisted with explant isolation, plating, and sample collection for RNA-seq. All other work described in this chapter was performed by the author. 


\section{Summary}

Somatic embryogenesis is a developmental process in which vegetative tissue becomes dedifferentiated from a more mature state and certain cells are subsequently reprogrammed to develop into structures similar to embryos formed by gamete fusion. These structures, called somatic embryos, are able to mature into clonal individuals. This tissue culture technique is commonly utilized for purposes such as regeneration of plants following genetic transformation and mass propagation of plants that are slow to mature. In genetic engineering of the cereal crop plant sorghum, application of the synthetic auxin 2,4-dichlorophenoxyacetic acid (2,4-D) plays a critical role in initiating the dedifferentiation of somatic cells and their transition into embryogenic callus tissue. However, the mechanism by which 2,4-D triggers this transition, including the molecular signaling and alterations in transcription it induces, is poorly understood. To investigate the timeline of gene expression changes induced by explant exposure to exogenous auxin, immature zygotic embryos were isolated from sterilized Sorghum bicolor seeds and cultured on callus induction media either with or without 2,4-D. The embryo tissue samples were collected at $6,12,24,36,48$, and 72 -hour time-points following tissue culture initiation and next-generation sequencing (RNA-seq) was used to profile transcriptional dynamics both across time and across conditions in response to 2,4-D treatment. Expression analysis revealed 4,818 differentially expressed genes across conditions, including 8 upregulated genes related to epigenetic modification, 251 upregulated transcription factors, and 123 upregulated hormone-related genes. Individual genes, includ- 
ing members of the bHLH and NAC transcription factor families, were singled out as candidates for further investigation into their potential role in somatic embryogenesis. Identifying important regulatory genes in somatic embryogenesis could lead to improvements in tools for enhancing plant regeneration following genetic engineering.

\section{Introduction}

Plant genetic modification will likely be an indispensable component of keeping global food production on pace with future projected population growth and environmental challenges posed by climate change (Altpeter et al. 2016). Genetic engineering tools offer increased speed and precision over conventional breeding methods, allowing for more timely responses to the changing biotic and abiotic pressures that affect crop production. Recently developed gene editing technologies, CRISPR in particular, enables targeted mutation of endogenous plant genes in a way that parallels conventional mutation breeding practices and opens up an avenue for GM crops to be brought to market without the time and expense required for regulatory evaluations as with previous genetically engineered crops (Kim and Kim 2016).

Constraints on the application of biotechnology in plant breeding include the often-low efficiency of exogenous gene delivery and integration into the target plant's nuclear genome, the amenability of cells or tissue carrying the exogenous material to be regenerated into a mature whole plant, and the genotype-specificity of plant transformation protocols (Kausch et al. 2019). Most established transformation procedures for crop plants rely on the controlled conditions of tissue culture to recover transgenic 
events. Somatic embryogenesis, a process that results in the development of a clonal individual plant originating from a single vegetative cell, is commonly employed to regenerate transgenic plants, especially monocots, through tissue culture. As with transformation efficiency, the capacity for regeneration via somatic embryogenesis to produce transgenic plants is highly variable by crop species and genotype. Methods that allow these limitations to be overcome will be required for biotechnology to be maximally utilized for trait improvement in a wide variety of plant species (Altpeter et al. 2016).

Somatic embryogenesis involves the reprogramming of non-reproductive plant cells, causing them to revert to a more immature developmental state (dedifferentiation) and then to proceed through a developmental pathway to form a somatic embryo, which is capable of maturing into a fertile individual plant (Yang and Zhang 2010). The developmental process is parallel to that of a zygotic embryo originating from the fusion of a male and a female gamete. However, unlike with zygotes, dedifferentiated cells undergoing somatic embryogenesis do not become genetically distinct from the parental germplasm through meiotic recombination. Thus, somatic embryos are clones of the plants from which they were derived. Somatic embryogenesis differs from other modes of regeneration used in tissue culture and to obtain transgenic plants (such as de novo shoot organogenesis) in that somatic embryos do not retain a vascular connection to the parental tissue and require different exogenous hormone application, as well as endogenous hormonal control, to induce (Elhiti et al. 2013).

To initiate the process of somatic embryogenesis, plant tissue that is competent to be transcriptionally reprogrammed is excised under sterile conditions and placed in 
tissue culture. This excised tissue is referred to as the explant and is often derived from an undifferentiated organ such as hypocotyl, cotyledon, shoot apical meristem or zygotic embryo (though leaf disks, stem internodes, petioles or root segments are occasionally used as well) (Liang et al. 2020). Upon induction by exogenous hormone application, somatic embryos develop either on the surface of explant tissue (direct somatic embryogenesis) or from a popcorn-like mass of undifferentiated tissue derived from the explant after a few days to several weeks in culture referred to as "callus" (indirect somatic embryogenesis). Though somatic embryos do not develop alongside an endosperm, they proceed through similar morphological phases as seen with zygotic embryogenesis (Fehér 2019).

Somatic embryogenesis in tissue culture can be induced on appropriate explant tissues by plant growth regulators, stress, or a combination of the two (Elhiti et al. 2013). The artificial auxin-mimicking compounds 2,4-D or NAA are perhaps most commonly used as amendments to somatic embryogenesis induction culture, while stress response may be induced by techniques such as heat-shock and deliberate explant wounding (Kumar et al. 2002, Santarem et al. 1997). These exogenous signals induce a cascade of molecular events that lead to wide-scale gene transcriptional changes which are necessary for cells within the explant tissue to be directed toward the embryogenic developmental pathway (Wang et al. 2020). This involves changes in endogenous biosynthesis and directional transport of the plant hormones auxin and cytokinin (and later gibberellins, ethylene, and abscisic acid), and changes in carbohydrate, lipid, and phenylpropanoid metabolism and fluxes of molecules involved in plant defense responses 
(Chen et al. 2020, de Carvalho Silva et al. 2014, Khan et al. 2015). Several regulatory genes have been identified as playing key roles in governing various aspects of the developmental shift during somatic embryogenesis (Elhiti et al. 2013, Horstman et al. 2017a). Broadly, these include genes operating the epigenetic level such as those involved in chromatin methylation as part of Polycomb repressive complex 2 (PRC2) and histone deacetylase (HDAC) genes (Bouyer et al. 2011, Tanaka et al. 2008), transcription factors of the AP2/ERF, B3 and MADS-box families such as BABY BOOM (BBM), LEAFY COTYLEDON 2 (LEC2), and AGAMOUS-LIKE 15 (AGL15) (Boutilier et al. 2002, Stone et al. 2001, Thakare et al. 2008). LEAFY COTYLEDON2 encodes a B3 domain transcription factor that induces embryo development, and hormone signaling, biosynthesis pathway, and transport genes such as INDOLE-3- ACETIC ACID INDUCIBLE 30 (IAA30), YUCCA 4 (YUC4), and PIN-FORMED 1 (PIN1) (Fehér 2015). Other genes that presumably play a more downstream role in somatic embryogenesis include the defense signaling cascade component MITOGEN-ACTIVATED PROTEIN KINASE 3 (MAPK3) (Zavattieri et al. 2010) and stress response genes such as the non-symbiotic hemoglobins $(H b 1 / 2)$ (Huang et al. 2014) and germin-like proteins (GLPs) (Mathieu et al. 2006).

Understanding key genes controlling the somatic embryogenesis pathway can lead to practical benefits such as improving plant regeneration capabilities. The genes LEAFY COTYLEDON 1 and $2(L E C 1 / 2)$ were identified in Arabidopsis thaliana as playing a role in plant embryogenesis and when each was ectopically expressed in transgenic tobacco plants, $L E C 2$ led to a marked increase in the quantity of somatic embryos and, 
subsequently, elongated shoots generated from callus tissue (Guo et al. 2013). The transcription factor $B A B Y B O O M(B B M)$, originally derived from Brassica napus, can enable the regeneration of fertile somatic embryos when transgenically expressed in sweet pepper (Capsicum annuum), a plant generally considered to be recalcitrant to transformation (Heidmann et al. 2011). Work by researchers at the company Corteva Agriscience over the last several years has led to development of a platform for achieving transformation through enhancement of somatic embryogenesis in many recalcitrant monocot crop genotypes using transgenic cassettes expressing the transcription factor Baby Boom (Bbm) and the shoot meristem regulator Wuschel 2 (Wus2), both derived from maize. Optimized co-expression of these genes allowed some level of transgenic plant recovery from every line of maize it was experimentally tested on as well as select lines of sorghum, rice, and sugarcane (Lowe et al. 2018, Lowe et al. 2016). In most cases, expression of these developmental regulators needs to be temporally controlled in order for them to be useful for transgenic plant production as their expression past the juvenile stage inhibits plant maturation and can cause developmental abnormalities (Gordon-Kamm et al. 2019). Identifying additional developmental genes that promote somatic embryogenesis could lead to the development of techniques for regenerating a wider variety of crop species and genotypes that remain recalcitrant to transformation. Grain sorghum (Sorghum bicolor) is the fifth-most produced cereal crop worldwide. In addition to being a staple food source in parts of Africa, South Asia, and Central America, it is also used as animal feed as well as a being a major source for biofuel production. It possesses innate traits that allow it to thrive in regions and soils where other 
major crops would fare poorly (Zuo et al. 2019). These traits include a heat and drought stress tolerance, an ability to produce at relatively high altitudes, and a tolerance for soil with high salinity or contaminated by some types of heavy metals (Abou-Elwafa et al. 2019). Together, these characteristics make sorghum a potentially highly climate change-resilient crop (Eggen et al. 2019). Some major production challenges to sorghum are insect pest such as the shoot fly (Satish et al. 2009) and chinch bug (Newman et al. 2010), and fungal pathogens such as Fusarium, Curvularia, and the ergot Claviceps sorghi (Chandrashekar and Satyanarayana 2006). 10-40\% of crop production loss is estimated to be attributable to arthropod pests and fungal and bacterial diseases, depending on the region (Repellin et al. 2001). In addition to addressing these biotic stressors, sorghum breeding has focused on improving nutritional content of the grain and making varieties more favorable for use in the biofuel production process (de Oliveira et al. 2018, Upadhyaya et al. 2019).

Genetic transformation has great potential for both trait improvement and for helping to elucidate genes contributing to favorable traits in sorghum. However, sorghum remains one the most recalcitrant to transformation of the major crop species. Different sorghum lines are extremely variable in their capacity for regeneration and only a handful of genotypes have been reported to be successfully transformed in the literature (Do et al. 2016, Howe et al. 2006, Liu and Godwin 2012, Nguyen et al. 2007, Wu et al. 2014). This may be in part due to the high content of phenolic compounds in some sorghum varieties, the release of which inhibits explant survival and regeneration 
in tissue culture (Nguyen et al. 2007). Though recent reports have shown some improvement in successful sorghum transformation using a maize $\mathrm{Bbm} / \mathrm{Wus} 2$ construct (Che et al. 2018, Kausch et al. 2019, Mookkan et al. 2017), this strategy does not provide the level a genotype-dependent transformation as has been reported in maize (Lowe et al. 2018). More work is required to uncover developmental regulator genes that can enable greatly improved transformation of a wide variety of sorghum genotypes.

Transcriptome profiling through sequencing of short cDNA reads (RNA-seq) has been used in multiple cases to study the reprogramming taking place during somatic embryogenesis. Yang et al. (2012) analyzed transcriptional dynamics taking place in cotton hypocotyl explants placed into tissue culture as well as embryogenic and non-embryogenic callus tissue. In addition to an upregulation of metabolic pathways and protein binding, the researchers found wide-scale alterations to the expression of genes involved in the biosynthesis, conjugate metabolism, transport, and signaling pathways of auxin. Another more recent study was carried out in the tropical tree species Dimocarpus longan (Chen et al. 2020). This group looked at gene expression during four distinct phases of callus and somatic embryo in tissue culture. They found that flavonoid biosynthesis was reduced in embryogenic callus versus non-embryogenic and that genes associated with fatty acid biosynthesis were upregulated in globular-stage somatic embryos. They also found significant upregulation of genes coding for extracellular proteins. Other RNA-seq studies associated with the somatic embryogenesis process were carried out in strawberry, coconut palm, and coffee (Gao et al. 2015, Quintana-Escobar et al. 
2019, Rajesh et al. 2016). Salvo et al. (2014) carried out the first RNA-seq study of somatic embryogenesis in a monocot crop species. This group looked at gene expression at time-points ranging from 0 to 72 hours after placing immature zygotic embryos of maize into tissue culture. By contrasting latter time-points with the initially isolated embryo control samples, a large array of stress response genes were found to be differentially expressed including germin-like proteins and glutathione-S-transferases. Orthologs of many other genes known to be involved in somatic embryogenesis in dicots such as $B B M, L E C 2$, WUS, CLAVATA (CLV), and AGAMOUS (AG) were also dynamically expressed over this time period. In the present study we aim to capture this same window of early transcriptional reprogramming for somatic embryogenesis in sorghum immature embryo explants. However, to disentangle transcriptional responses due to tissue dedifferentiation prior to somatic embryo formation from gene expression changes resulting only from extended time in tissue culture, we made use of a parallel control condition at each time-point where explants are cultured without the presence of the plant growth regulator 2,4-Dichlorophenoxyacetic acid (2,4-D), which is necessary for somatic embryogenesis to take place in the sorghum regeneration system.

\section{Results}

\section{Global gene expression analysis}

The public sorghum line 'P898012' was chosen for studying somatic embryogenesis as it is considered a highly regenerable genotype and is routinely used in transfor- 
mation (Do et al. 2016). Total RNA samples were extracted from immature embryo explant tissue at seven different time-points after being placed on sorghum callus induction medium (CIM) following isolation from greenhouse-grown immature seeds. The time-points used for sample collection were 0 hours (collected immediately after explant isolation, before placement on CIM) and 6, 12, 24, 36, 48, and 72 hours following incubation in tissue culture. For each of the six time-points following 0 hours, two separate samples were collected: one from a standard sorghum CIM plate containing 1.5 $\mathrm{mg} / \mathrm{L}$ of 2,4-D, which were designated with [+], and a control sample from a modified CIM with 2,4-D omitted as a component, which were designated with [-]. Each tissue sample consisted of a pool of about 25 explants and samples for each discrete treatment-time combination were collected in three biological replicates, for a total of 39 individual tissue samples (see Figure 2.1 for illustration).

Across the 39 samples, a total of approximately 1.01 billion raw reads were generated, ranging from $19,099,251$ to $33,626,422$ reads per sample with a mean of $25,864,486$ reads per sample. Trimming these raw reads for quality led to the removal of $1.7 \%$ of these reads, while removal of mitochondrial and ribosomal RNA sequences led to another $9.8 \%$ reduction of the total raw reads count. Aligning reads to the sorghum reference genome (v3.1.1) (McCormick et al. 2018) yielded a uniquely mapping read rate of $91.0 \%$ and an overall alignment rate of $94.6 \%$. Aligned reads were assembled into transcripts using the previously annotated reference transcript set as a guide, which consisted of 34,129 annotated genes and 47,121 unique transcripts. The transcript assembler also generated novel transcripts based on reads not assigned to one of 
the mRNAs of the annotated genes. These novel sequences were isolated from each sample alignment, merged together, and then analyzed and filtered to remove those that either matched, contained, were contained by, or otherwise significantly overlapped with genes from the reference annotation. This resulted in 3,179 additional transcripts, which were appended to the reference annotation set, and the transcript assembly process was repeated using the combined set of reference and putative novel transcript annotations as a guide by the assembler. Values for both raw read counts and FPKM (fragments per kilobase of transcript per million mapped reads) at the gene level were computed for each sample dataset to use in different downstream analyses. $94.7 \%$ of uniquely mapping reads were assigned to a gene by read count summation. See Table 2.1 for a summary of the read pre-processing, alignment, and transcript assignment rates for each sample.

\section{Differential expression analysis}

Sets of FPKM values from each sample were normalized across samples and then used to generate a matrix consisting of Pearson correlation values from pairwise comparisons of all samples (data not shown). At the same time, raw read counts were combined into a single data object, which was then filtered for genes that had read counts of greater than 5 in at least two of the samples. Values were normalized using the TMM method and the resulting set was plotted using multidimensional scaling (MDS) (Figure 2.2). Two issues with the data became clear from analyzing both the correlation matrix and the MDS plot. Firstly, the samples taken at 0 hours, though highly correlated with 
each other, were notably distant from all other samples due to differences in the handling of explants prior to collection, which was anticipated before the RNA-seq data was analyzed. Secondly, samples from biological replicate number two taken at 6, 12, 24, and 36 hours in the 2,4-D[-] condition were much more closely correlated with samples from the 2,4-D[+] treatment than with others in the 2,4-D[-] condition at the corresponding time-points. This was likely due to mislabeling of media plates during the sample collecting process or even to a mistake when formulating the media. After uncovering these issues, a decision was made to eliminate all 0 hour samples from differential expression analysis going forward and to eliminate samples taken for the second experimental replicate at the $6,12,24$, and 36 -hour time-points from analysis, leaving two replicates for each sample at those times. Since there were no apparent issues with any samples at the 48 and 72 -hour time-points, samples from all three experimental replicates at these times were still included in the analysis.

Differential expression analysis was performed using the modified dataset as described. Samples were grouped according to treatment by time-point and were fitted to a generalized linear model. Contrasts were made both across time (i.e., comparing 12 , $24,36,48$, and 72-hour time-points in each condition to expression at their corresponding 6-hour time-point) and across conditions (i.e., comparing each time-point in the 2,4$\mathrm{D}[+]$ treatment to expression at the corresponding time-point in the control 2,4-D[-] condition). The resulting sets were each filtered for genes showing at least a 2-fold change in expression and an adjusted $P$-value below 0.05 . 


\section{Comparisons made through time within conditions}

For the across time comparisons, 9,011 genes in total were found to be differentially expressed in at least one contrast in either the $2,4-\mathrm{D}[+]$ or the $2,4-\mathrm{D}[-]$ condition (See figure 2.3 for a summary of each DE set). 4,193 (46.5\%) of these were differentially regulated over time in both of the conditions including 1,694 genes downregulated in both conditions and 2,502 genes upregulated in both conditions. Common transcriptional regulation patterns of genes across time between both conditions is likely to be caused by the artificial conditions of tissue culture rather than capturing genes involved in the embryogenic developmental process. Thus, these genes were dropped from further analysis. 2,950 genes were differentially regulated across time in only the control condition, including 1,369 downregulated and 1,595 upregulated. Unique to the 2,4-D[+] treatment condition across time, 2,243 genes were differentially expressed, including 1,259 that were downregulated and 998 that were upregulated. 203 genes were upregulated through time in the control condition, which were simultaneously downregulated in the 2,4-D[+] treatment condition. 96 genes were downregulated in the control condition while being upregulated in the 2,4-D[+] treatment condition. These latter two sets were saved to be compared to sets from the across-condition contrasts.

At the 12-hour vs. 6-hour 2,4-D[+] comparison, the single-most upregulated and downregulated sequences were those named Sobic.new.1275 (at a $\log _{2}$-fold change of 9.60) and Sobic.new.5133 (at a $\log _{2}$-fold change of -13.52), respectively. Both of these were novel annotations for immature embryo tissue generated during the transcript assembly process, however, neither of these annotated sequences code for a protein 
product and neither of them have a close relationship to other known nucleotide sequences based on a BLASTn search. Throughout the across-time contrasts in both the 2,4-D[+] and 2,4-D[-] conditions, novel transcript annotations are overrepresented among the genes showing the highest degree of differential expression from the 6-hour time-point. Some of these sequences have BLASTn hits to previously annotated sorghum genes or genes from other closely-related organisms such as Zea mays and Setaria viridis, but few of these sequences are protein-coding. Some of them might therefore represent long non-coding RNAs, which may play some role in the early somatic embryogenesis response. See Tables 2.2 and 2.3 for a list of the most dramatically differentially expressed genes in the across-time comparisons.

Iso-temporal comparisons made between conditions

For the cross-condition comparison set, 4,818 genes were differentially expressed 2-fold or greater in at least one contrast, including 2,118 upregulated and 2,810 downregulated in the 2,4-D[+] treatment compared to the 2,4-D[-] control. Total differentially expressed genes in each contrast ranged from 663 (468 upregulated and 195 downregulated) at the 6-hour time-point, to 3,564 (1,321 upregulated and 2,243 downregulated) at the 72-hour time-point (See Table 2.4). 158 genes were upregulated in contrasts made at all six time-points, while 112, 207, and 295 genes were upregulated in five, four, or three of the contrasts, respectively. 36 genes were downregulated in all six contrasts, while 72,257 , and 478 were downregulated in five, four, or three of the contrasts, respectively. 
The most highly upregulated gene in the treatment condition at the 6-hour timepoint was Sobic.010G052700 at a $\log _{2}$-fold change of 10.90 , which was functionally annotated as a member of the AUX/IAA family of transcriptional regulators. The most downregulated gene at that time-point was Sobic.005G127800 at a $\log _{2}$-fold change of 6.32 , annotated as a disease resistance protein of the LRR family. Additional top upregulated genes of note in each contrast included Sobic.003G306500 ( $\left.\log _{2} \mathrm{FC}=10.55\right)$, a member of the GH3 family of auxin-responsive proteins at 12 hours in tissue culture, Sobic.new.2302 ( $\left.\log _{2} \mathrm{FC}=12.70\right)$, most closely related to the predicted sorghum serine/threonine-protein kinase SAPK1 at the 24-hour time, Sobic.002G253000 $\left(\log _{2} \mathrm{FC}=\right.$ 8.81), annotated as a NAC domain containing protein family member at 36 hours, and at 48 hours, Sobic.009G179000 $\left(\log _{2} \mathrm{FC}=9.08\right)$, an ortholog of BRASSINOSTEROID INSENSITIVE 1-associated receptor kinase 1 (BAK1) in rice, also known as SOMATIC EMBRYOGENESIS RECEPTOR KINASE 3 (SERK3). The most highly downregulated genes at the contrasts past 6 hours were Sobic.new.5133 $\left(\log _{2} \mathrm{FC}=-14.25\right)$, which most closely aligned to the predicted sorghum ortholog of arabinogalactan protein 1 at 12 hours, Sobic.new.230 $\left(\log _{2} \mathrm{FC}=-12.70\right)$, whose closest ortholog was the maize uncharacterized protein LOC111347717 at 24 hours, Sobic.new.1245 ( $\left.\log _{2} F C=-8.77\right)$, coding for an O-methyltransferase protein at 36 hours, and Sobic.004G292900 ( $\left.\log _{2} \mathrm{FC}=-12.54\right)$, a protease inhibitor/seed storage protein of the LTP family at 48 hours. See Tables 2.5 and 2.6 for a list of the most dramatically differentially expressed genes in the cross-condition comparisons. 


\section{GSEA of cross-condition contrasts}

Gene set enrichment analysis was performed using separated groups of upregulated and downregulated genes for each cross-condition contrast. The gene sets selected for analysis were Gene Ontology (GO) biological process and molecular function terms, gene families (Gfam), and PlantCyc and KEGG gene pathway annotations. Only categories that were significantly overrepresented in at least one set were used for comparison. Furthermore, categories that were redundant with others in the same annotation set, had an adjusted $P$-value near the significance threshold in only one or two groups, or were otherwise uninteresting, were eliminated by manual inspection, leaving a total of 56 overrepresented terms. The results are graphically represented in Figure 2.4 .

The most highly overrepresented terms among the sets of upregulated genes were the GO biological process terms: auxin-activated signaling pathway (GO:0009734), regulation of transcription, DNA-templated (GO:0006355), regulation of translation (GO:0006417), and response to auxin (GO:0009733). Other interesting overrepresented biological process categories included: cellular response to water deprivation (GO:0042631), de-etiolation (GO:0009704), glutathione metabolic process (GO:0006749), para-aminobenzoic acid metabolic process (GO:0046482), phosphorelay signal transduction system (GO:0000160), response to cytokinin (GO:0009735), response to herbicide (GO:0009635), and shoot system morphogenesis (GO:0010016). For 
these categories, the overrepresentation was most pronounced very early in tissue culture with the highest values at the 6-hour time-point and either decreasing or non-significant at the 12 and 24-hour times.

The GO molecular function terms most overrepresented among the sets of upregulated genes included: indole-3-acetic acid amido synthetase activity (GO:0010279), protein dimerization activity (GO:0046983), transferase activity, transferring hexosyl groups (GO:0016758). Terms with a lesser degree of overrepresentation, mainly in the earlier time-points were: auxin efflux transmembrane transporter activity (GO:0010329), benzaldehyde dehydrogenase (NAD+) activity (GO:0018479), glutathione binding (GO:0043295), phosphorelay response regulator activity (GO:0000156), quercetin 7-O-glucosyltransferase activity (GO:0080044) and signal transducer activity (GO:0004871). Interestingly, the molecular function term "sequence-specific DNA binding transcription factor activity" (GO:0003700) was the only term that was most highly overrepresented in the last three time-points among the sets of upregulated genes.

The gene family sets that were most highly overrepresented among upregulated genes included the AUX/IAA transcriptional regulators, followed by the GT1 subfamily of carbohydrate-active enzymes, NAC family transcription factors, the cytochrome p450 subfamily CYP81A, AP2/ERF family transcription factors, LOB family transcription factors, and bHLH family transcription factors. Unsurprisingly, the early auxin responsive AUX/IAA genes were the most dramatically overrepresented family immediately beginning at the 6-hour time-point, gradually decreasing in the degree of their overrepresen- 
tation over time. The GT1 family, the CYP81A subfamily were significantly overrepresented at only first three time-points suggesting an early role for these genes. The NAC family was, similarly, most overrepresented at the 6-hour time-point, decreasing from then on and tracking closely the level of overrepresentation of the AUX/IAA set. The LOB and bHLH transcription factor families were both significantly overrepresented early on in the time-course, both cresting at 12 hours. The bHLH family decreased in overrepresentation after the early time-points, while the LOB family reached its highest level at 72 hours. Interestingly, the AP2/ERF family which includes the BBM gene and several relatives known to play a role in inducing somatic embryogenesis (Horstman et al. 2017b), was not significantly overrepresented until the 48 and 72 -hour time-points, suggesting a later role for these genes than the other transcription factor families that were more rapidly expressed after 2,4-D induction.

The annotated KEGG pathways that were overrepresented among upregulated genes were "plant hormone signal transduction" and "glutathione metabolism." Genes falling into the hormone signal transduction pathway were mostly AUX/IAA genes associated with the early auxin response and thus closely tracked the overrepresentation levels of that gene family. The glutathione metabolism category was only significantly overrepresented in the 6 and 12-hour time-points, indicating that this represents a very early response induced by 2,4-D. The PlantCyc annotations that were overrepresented included "detoxification of reactive carbonyls in chloroplasts" and "tryptophan degradation VI (via tryptamine)." Both of these were only significantly altered in the 6-hour 
time-point. They share several overlapping genes, at least a few of which are likely involved in biosynthesis of endogenous auxin, which is a positive feedback response known to be caused by application of exogenous auxin such as 2,4-D.

Several categories were also overrepresented among downregulated genes in the cross-condition contrasts. The downregulated genes in this context are equivalent to those that would be upregulated when inverting the analysis by contrasting the control condition against the treatment condition. In the absence of 2,4-D inducing tissue dedifferentiation and callus formation, immature embryos placed on the control medium essentially begin to progress through the default developmental sequence as both the radicle and coleoptile begin to extend from the abaxial surface of the embryo and become much more pronounced by the 72-hour mark than on explants incubating on 2,4 D-containing medium at the equivalent time post-isolation. Thus, it is to be expected that there would be overrepresentation within gene sets associated with differentiation and maturation of tissues among the total downregulated genes in the cross-condition contrasts. GO biological process terms such as: chlorophyll biosynthetic process (GO:0015995), plastid organization (GO:0009657), fatty acid biosynthetic process (GO:0006633), lipid transport (GO:0006869), rRNA processing (GO:0006364), and starch biosynthetic process (GO:0019252) as well as molecular function terms including: NADPH dehydrogenase activity (GO:0003959), transporter activity (GO:0005215), and peroxidase activity (GO:0004601) were all significantly overrepresented among downregulated genes. The only transcription factor family that was significantly enriched in the downregulated gene groups was the WRKY family and only at the 6-hour time-point. 
The KEGG terms "photosynthesis," "phenylalanine metabolism," and "linoleic acid metabolism," and the PlantCyc terms "phenylpropanoid biosynthesis," "oxygenic photosynthesis," and "flavonoid biosynthesis" denoted the overrepresented pathways among the downregulated gene set.

Gene mining based on important gene functional classes

Taking into account what has been discovered so far about the role of genes in somatic embryogenesis, there is evidence that this complex process is regulated at least at three levels: epigenetic (DNA and chromatin modifying proteins), transcriptional, and through hormone pathways (Horstman et al. 2017a). Several genes falling into one or more of these broad categories have been experimentally validated as having at least some functional control over somatic or zygotic embryogenesis. Therefore, to assess how well this RNA-seq study in sorghum captures the significance of these known genes and to discover new candidate genes tentatively functioning at each level, curated sets of all putative epigenetic modulator, transcriptional regulator, and hormone-related genes in the sorghum genome were compared against sets of genes that were differentially expressed across conditions.

Epigenetic modulators. Protein sequences of epigenetic modulator (EM) genes in Arabidopsis thaliana and Oryza sativa were downloaded from The Arabidopsis Information Resource (TAIR) database and the Rice Genome Annotation Project, respectively. Following the process of curation outlined in Silva et al. (2020), these sequences were 
aligned to the sorghum proteome using BLAST-P and the top 5 hits for each sequence were extracted and then sorted and filtered to remove duplicates. At the same time, profile hidden Markov models (HMMs) for protein domains common to each functional category of epigenetic modulator were downloaded from the InterPro website and used to search the sorghum proteome with HMMer (Eddy 2011). The consensus list generated from the intersection of the resulting sets consisted of 100 putative EM genes. Only 3 genes from this set were contained within the set of downregulated genes: Sobic.004G255500, Sobic.002G199000, and Sobic.009G251000, all of which were annotated as containing the SET domain (InterPro ID: IPR001214), which is associated with lysine-specific methyltransferase activity. However, the closest BLAST hits to these genes in Arabidopsis (AT3G07670, LSMT-L, and PTAC14, respectively) were annotated as having rubisco methyltransferase activity, a set of photosynthetic and chloroplast development genes for which none of the members of the family are known to make covalent modifications to histones. Therefore, none of these may represent actual EM genes.

On the other hand, 8 genes of the putative EM set were present in the set of upregulated genes. These included two DNA-modifying proteins: Sobic.001G188500, an ortholog of the Arabidopsis DEMETER (DME) which is a DNA glycosylase involved in a demethylation mechanism, and Sobic.001G055700, an ortholog of DNA methyltransferase 1 (MET1). 5 of the genes (Sobic.001G175000, Sobic.001G284400, Sobic.002G193300, Sobic.004G006600, and Sobic.004G338500) were annotated as having histone demethylase function as each contained the Jumonji C (jmjC) domain. Finally, a single gene, Sobic.003G396600, was an ortholog of Arabidopsis HISTONE DEACETYLASE 3 (HDA3). For 
each of these genes, significant expression differences were mainly seen at the 36,48 , and 72-hour time-points. Figure 2.5 displays expression plots for these genes.

DNA binding transcription factors. Transcription factor protein sequences from Arabidopsis, maize, and rice were downloaded from the Plant Transcription Factor database v3.0 (PInTFDB; http://plntfdb.bio.uni-potsdam.de/v3.0/), which were aligned to the sorghum proteome with BLAST-P to extract possible orthologous sequences. Multiple sequence alignment files for each of the protein domains defining placement into the transcription factor families, also obtained from PInTFDB, were used to build HMMs which were then scanned against the sorghum proteome and the criteria outlined by PInTFDB for inclusion in each of the transcription factor families were followed to categorize each of the candidate sorghum genes. Gene sets obtained by domain scanning were compared to the set of BLAST-P results, which resulted in a set of 2,944 putative sorghum transcription factor genes. Family-wise subsets of these genes were then compared with each set of differentially expressed genes in the cross-condition comparisons.

Among the set of differentially expressed genes in the cross-condition contrasts at any time-point 282 unique transcription factors from 40 different families as annotated in the PInTFDB v3.0 database were downregulated. The highest numbers of these were from the MYB, bHLH, WRKY, bZIP, AP2/ERF, G2-like, C2H2, and NAC domain-containing families. The WRKY transcription factor genes Sobic.001G381300, So- 
bic.002G202700, Sobic.002G202800, Sobic.002G418500, Sobic.008G060300, and Sobic.009G238200, were among the earliest individual genes to show a significant decrease in expression compared to the control condition at the 6-hour time-point. Expression plots for these WRKY-related genes are displayed in Figure 2.6. In the latter time points, several MYB-related transcription factors are downregulated, including Sobic.003G136600 and Sobic.004G273800, which are differentially expressed in five of the six contrasts.

The transcription factor families most abundantly represented in the set of upregulated genes were AP2/ERF (which includes the known somatic embryogenesis inducer $B B M), b H L H, N A C, L O B, A R F$, and bZIP. It is noteworthy that most of these same families were also the most represented among downregulated genes, which is consistent with different members of the same family playing opposite transcriptional roles. 10 of the 27 sorghum genes annotated as auxin response factors were upregulated at some time-point with 6 of them being differentially expressed in three or more contrasts. Several of the 39 members of the AP2/ERF family that were upregulated at some time-point shared a similar expression pattern to that of the sorghum ortholog of $B B M$. Expression plots for select upregulated AP2/ERF family members are shown in Figure 2.7. A surprising number of bHLH transcription factors were induced early in the timecourse. Out of 21 total upregulated bHLH genes, 8 of them were upregulated at all six time-points. A set of transcription factors of the NAC family were also among those induced very early in the time-course with 13 out of 20 of them being upregulated at the 
6-hour time-point. 6 of these genes were differentially expressed in all six contrasts. Expression plots for select upregulated bHLH and NAC domain family genes are displayed in Figure 2.8.

The LOB transcription factor family, for which 13 of the 35 members in sorghum were upregulated included 7 that were significantly upregulated in at least three contrasts. For the bZIP transcription factors, one of them (Sobic.005G041100) was upregulated in all six contrasts. Other transcription factor genes of note that were strongly upregulated include the $\mathrm{C} 2 \mathrm{H} 2$-Dof transcription factor Sobic.001G166200, the $\mathrm{C} 2 \mathrm{H} 2$ factor Sobic.001G020200, the LFY domain-containing protein Sobic.010G052700, the heatshock transcription factor Sobic.005G041100, the MYB-like transcription factor Sobic.003G129000, the OFP family factor Sobic.001G468900, the two TCP domain-containing factors Sobic.010G253300 and Sobic.004G037800, and the trihelix domain-containing Sobic.003G129000, all of which were upregulated in every contrast. Expression plots for select upregulated members of the LOB family and other notable transcription factor genes are displayed in Figure 2.9.

Other transcriptional regulators. Gene family members for other transcriptional regulators in sorghum were again procured using sets obtained from PInTFDB and following the same pipeline. Among the set of downregulated genes were Sobic.009G182000, an ARID family protein differentially regulated at the last 3 time-points, Sobic.006G135100 and Sobic.010G015800, two PHD family members differentially regulated in four and all contrasts, respectively, and Sobic.009G203700 and Sobic.003G035700 (best Arabidopsis 
hit for both was IAA26/PAP1), two AUX/IAA family genes downregulated at the last four and the last two time-points, respectively. As for upregulated genes, AUX/IAA genes were by far the most represented family with 18 out of 27 members in the sorghum genome upregulated in at least one contrast. 10 of these genes were upregulated at every time-point, while another 3 were upregulated in five contrasts. In addition to the AUX/IAA family, 5 PHD family proteins were upregulated in three or more contrasts as well as two GNAT transcriptional co-activator family genes, Sobic.001G079600 and Sobic.010G210100, which were upregulated in three and four different contrasts, respectively. In addition, a single Jumonji family protein, Sobic.004G338500, described earlier as a putative epigenetic modulator with a histone demethylase function, was upregulated at the final three time-points.

Hormone-related genes. To search for sets of genes related to hormone pathways the Quick GO webpage (https://www.ebi.ac.uk/QuickGO/) (Binns et al. 2009) was used to obtain Gene Ontology terms associated with seven different plant hormones: abscisic acid, auxin, brassinosteroids, cytokinin, ethylene, gibberellin, and jasmonic acid. The collected GO terms were then used as queries to retrieve lists of sorghum genes associated with each term using Biomart (http://plants.ensembl.org/biomart/martview/) (Smedley et al. 2015). These lists were compared with the upregulated and downregulated gene sets.

Among downregulated genes, 28 related to abscisic acid were downregulated at some point, along with 25 related to auxin, 5 related to brassinosteroids, 20 related to 
cytokinin, 9 related to ethylene, 14 related to gibberellin, and 5 related to jasmonate. The 6 genes related to abscisic acid downregulated at three or more time-points were annotated with the GO biological process terms "abscisic acid-activated signaling pathway," "regulation of abscisic acid biosynthetic process," and "response to abscisic acid." The 7 auxin-related genes downregulated at multiple time-points included two orthologs of ILR1 (Sobic.001G021900 and Sobic.002G093500), a peptidase involved in the hydrolysis of IAA-amino acid conjugates, an ortholog of AS1 (Sobic.008G137500), which was annotated as auxin-responsive, and Sobic.009G203700 one of the orthologs of Arabidopsis IAA26/PAP1, the auxin signaling protein also noted earlier as the only consistently downregulated member of the AUX/IAA family in this experiment, when the majority of members were strongly upregulated. Other notable hormone-related genes that were downregulated include: Sobic.004G094100, an ortholog of GNC/GATA21 responsive to cytokinin and a modulator of glutamate synthase expression and chlorophyll biosynthesis, Sobic.004G313100, an ortholog of ACO4 or 1-AMINOCYCLOPROPANE-1-CARBOXYLATE OXIDASE which catalyzes the final step of ethylene biosynthesis, an ortholog of ATGA2OX1 (Sobic.003G022700) an enzyme involved in gibberellin catabolism, and an ortholog of MYB4 (Sobic.002G279100), a transcription factor regulating phenylpropanoid metabolism that is responsive to jasmonic acid.

For the sets of upregulated genes in any time-point contrast, 29 were related to abscisic acid, 60 were related to auxin, 10 related to brassinosteroids, 11 related to cytokinin, 4 related to ethylene, 9 related to gibberellin, and 5 related to jasmonate. The 
subset of upregulated auxin-related genes included, as noted previously, several members of the AUX/IAA and ARF families involved in the auxin-activated signaling pathway, in addition to genes annotated with the GO biological process terms "auxin polar transport," "auxin homeostasis," and "auxin efflux." These include genes orthologous to ATPIN1, ATPIN3, and ATPIN4 (Sobic.010G095100, Sobic.004G240900, So-

bic.003G235800, and Sobic.005G025900) in addition to the auxin catabolizing enzyme DAO1 (Sobic.006G118400 and Sobic.004G149100), several putative members of the SAUR-like family of auxin-responsive proteins (Sobic.007G020800, Sobic.010G027700, Sobic.002G285950, Sobic.002G285600, Sobic.002G285450, Sobic.002G285300, and Sobic.001G184800), and an ortholog of ENHANCER OF PINOID (ENP) (Sobic.010G066100), an NPH3-like protein involved in the development of cotyledons. Expression plots for various notable auxin-related genes are displayed in Figure 2.10.

Abscisic acid-related genes that were upregulated included those classed into the GO terms "abscisic acid-activated signaling pathway," "abscisic acid transport," and "response to abscisic acid." Specific genes differentially expressed in three or more contrasts included an ortholog of the Arabidopsis LEC1-Like (L1L) (Sobic.004G254500), which is a regulator of embryo development, an ortholog of the abscisic acid exporter ATABCG25 (Sobic.005G061000), and orthologs of the protein phosphatase 2C genes ABA-hypersensitive germination 1 (AHG1) and highly ABA-induced PP2C protein 3 (HA/3) (Sobic.006G018400 and Sobic.009G238600, respectively), both involved in the seed germination process. Genes related to cytokinin that were upregulated at three or more 
time-points included genes potentially involved in cytokinin catabolism (Sobic.003G421100 and Sobic.006G151500; orthologs of ATCKX1 and ATCKX6, respectively) and in cytokinin biosynthesis (Sobic.001G217700 and Sobic.009G254900; orthologs of LOG3 and LOG7, respectively) as well as Sobic.006G272600, an oxalate-CoA ligase enzyme responsive to cytokinin. Other hormone-related genes of note that were upregulated in at least three contrasts include: Sobic.010G091700, an ortholog of Arabidopsis $K D R$, which is a bHLH family transcription factor responsive to brassinosteroid induction and Sobic.003G379500, Sobic.006G150800, and Sobic.009G077500, all genes involved in the gibberellin metabolic process (orthologs of ATGA200X1, ATGA2OX8, and ATGA2OX1, respectively). Expression plots for select genes related to hormones other than auxin are displayed in Figure 2.11.

Other functional categories. To more closely investigate differentially regulated genes involved in processes other than epigenetic and transcriptional regulation and hormone pathways, individual genes associated with GO terms that were overrepresented among the sets of differentially expressed genes and unrelated to the functions mentioned above were retrieved and compared against the downregulated and upregulated gene sets. The GO descriptions searched were the biological process terms "reactive oxygen species metabolic process," "cellular response to water deprivation," "de-etiolation," "glutathione metabolic process," "para-aminobenzoic acid metabolic process," "phosphorelay signal transduction system," and "shoot system morphogenesis," the molecu- 
lar function terms "benzaldehyde dehydrogenase (NAD+) activity," "NADPH dehydrogenase activity," "phosphorelay response regulator activity," "protein dimerization activity," and "transferase activity, transferring hexosyl groups," in addition to the Gfam category "carbohydrate-active enzymes, subfamily GT1." The only two of these categories represented among genes downregulated in at least three contrasts were "reactive oxygen species metabolic process" and "NADPH dehydrogenase activity." 13 genes belonged to the first category and all encoded members of the peroxidase protein superfamily. Of the 4 genes belonging to the second category, 2 of them (Sobic.001G206200 and Sobic.006G268200) were involved in the biosynthesis of chlorophyll, whereas the 2 others (Sobic.003G431700 and Sobic.010G005500) encoded ferredoxin-NADP[+]-oxidoreductases (orthologous to $F N R 1$ and $F N R 2$, respectively).

Among the set of genes upregulated in at least three conditions, the 4 genes associated with the "reactive oxygen species metabolic process" were again all peroxidase superfamily proteins. The genes involved with "cellular response to water deprivation" were Sobic.002G173200, an ortholog of glutathione S-transferase TAU 28, and two UDPglucosyltransferase enzymes (Sobic.002G265600 and Sobic.002G265800), the first of which, orthologous to UGT74E2, acts on the auxin compound IBA. These two genes were also included in the "para-aminobenzoic acid metabolic process," "shoot system morphogenesis," "transferase activity, transferring hexosyl groups," and "carbohydrateactive enzymes, subfamily GT1" categories. Genes associated with the category de-etiolation were 4 glutathione S-transferases (Sobic.001G317600, Sobic.001G318200, Sobic.001G319500, and Sobic.001G319600), which all overlapped with the "glutathione 
metabolic process" category, and the auxin signaling gene IAA4 (Sobic.002G273400). The "protein dimerization activity" category contained many of the AUX/IAA and ARF family members noted previously as well as an ortholog of CIB1 (Sobic.004G267400), a $\mathrm{bHLH}$ transcription factor involved in floral development, the ortholog of $K D R$ as noted previously, and an ortholog of SPATULA (Sobic.010G051800), another bHLH transcription factor involved in regulating meristem size. The GT1 carbohydrate-reactive enzyme family and the "transferase activity, transferring hexosyl groups" categories were mostly overlapping and contained many UDP-glucosyltransferase genes in addition to the two noted previously. The "benzaldehyde dehydrogenase (NAD+) activity" category revealed an ortholog of AAO2 (Sobic.001G062600), an enzyme putatively involved in the biosynthesis of abscisic acid. The "phosphorelay signal transduction system" and "phosphorelay response regulator activity" categories were completely overlapping and included five genes (Sobic.004G178000, Sobic.006G090300, Sobic.006G263300, Sobic.005G030500, and Sobic.008G012300) orthologous to ARABIDOPSIS RESPONSE REGULATOR genes including $A R R 3, A R R 6, A R R 8$, and $A R R 9$. Finally, the "shoot system morphogenesis" category included the LOB domain transcription factor Sobic.001G147000, an ortholog of $A S L 5$, which is also involved in leaf development, and Sobic.006G169200, an ortholog of the homeobox-leucine zipper transcription factor HAT3.

Comparison of differentially expressed genes to results from other studies

The sorghum orthologs of several genes known to have regulatory control over somatic embryogenesis were compared to the temporal expression of maize orthologs 
of these genes reported in Salvo et al. (2014). The researchers reported that orthologs of the Wuschel-related homeobox (WOX) genes WOX5 and WOX11 were upregulated over 8-fold across time and WOX2 was downregulated over 8-fold across time. In this study, we saw more or less the same trends, as the WOX5 and WOX11 orthologs (Sobic.003G360200 and Sobic.001G386600, respectively) were both upregulated in crosscondition comparisons at the latter time-points, while the WOX2 ortholog (Sobic.003G350900) was not significantly differentially expressed across conditions but was downregulated across time. In contrasts to the finding in maize, the sorghum ortholog of homeobox gene KNOTTED1 (Sobic.001G106200), was upregulated across time in the 2,4-D[+] condition and trended upward over the control condition at the last two timepoints, though it did not clear the 2-fold change threshold. The sorghum ortholog of WUS (Sobic.006G254900), though there was an apparent difference in expression between two conditions with the treatment displaying higher overall read counts, was not among the set of significant differentially expressed genes. This is probably due to lack of statistical power for comparisons with this gene since read counts in the samples were very low overall and were completely absent from most samples in the control condition. Expression plots for sorghum orthologs of these homeodomain proteins are displayed in Figure 2.12.

Salvo et al. (2014) also reported modest increases in expression of ZmSERK2 and ZmSERK3 from the 0-hour to the 24-hour time-point. The orthologous genes in sorghum (Sobic.006G104500 and Sobic.004G189900, respectively) showed an overall downward trend in both conditions. However, two other apparent SERK relatives in sorghum 
showed interesting trends. Another sorghum ortholog of Arabidopsis SERK2 (Sobic.007G059600) did show a modest increase in expression in the treatment condition over the control, though the difference never reached more than a $50 \%$ increase in expression. Meanwhile, Sobic.009G179000, annotated as an ortholog of the Oryza sativa SERK3/BRASSINOSTEROID INSENSITIVE 1-associated receptor kinase 1 (BAK1), showed upregulation in five of the six cross-condition contrasts and was among the top 10 most highly upregulated genes at the 48 and 72 -hour time-point comparisons. The sorghum ortholog of ZmLEC1 (Sobic.004G254500) was upregulated in the latter four time-point contrasts with expression trending downward over time in the control condition while remaining mostly stable in the treatment condition, while in Salvo et al. (2014) ZmLEC1 was reported to decrease in expression over time. The sorghum ortholog of LEC2 (Sobic.007G000400), on the other hand, was actually expressed at a lower level at all times in the treatment condition than in the control, though it was not significantly differentially expressed at any time. It was also reported in Salvo et al. (2014) that a maize ortholog of the MADS-box transcription factor AGAMOUS-LIKE 15 (AGL15) was upregulated over 8-fold through time, but in this study, no differential regulation of the putative sorghum AGL15 ortholog or any of its close relatives was detected.

It was also reported that several glutathione S-transferase (GST) genes in maize were upregulated through the time-course. However, in sorghum it was found that only orthologs of GST37 and GST8 (Sobic.001G317600 and Sobic.001G164800, respectively), were consistently upregulated, though the latter of these trended downward in both conditions over time. GST15 (Sobic.009G043600) and GST34 (Sobic.001G319500) were 
both upregulated across conditions at the 6-hour time-point, and the former at 12 hours as well, but their expression levels of the treatment condition roughly tracked those of the control condition after that. Some GSTs including Sobic.001G310300, Sobic.001G514100, Sobic.001G514200, Sobic.001G514300, Sobic.001G514400, and Sobic.002G283500 (orthologs of GST14, GST10, GST9, GST12, GST11, and GST16, respectively), were sharply downregulated across conditions beginning at the 48 or 72 -hour time-points. Only 1 ortholog of the germin-like proteins discussed in Salvo et al. (2014) was significantly upregulated across time. In the present study this gene, GLP2-1 (Sobic.006G018100), was upregulated through time in both conditions until the 72-hour mark when it was upregulated over 5-fold across conditions.

$B B M$ is thought to be a major regulator of somatic embryogenesis. It has partial functional redundancy with several other genes in the AP2/ERF family of transcription factors and in Arabidopsis is known to directly and indirectly regulate the expression of many other genes involved in zygotic and somatic embryogenesis including the set of regulators $L E C 1, A B I 3, F U S 3$, and $L E C 2$, collectively termed the $L A F L$ network (Horstman et al. 2017b). The authors of this study used chromatin immunoprecipitation/sequencing (ChIP-seq) to identify potential transcriptional targets of $B B M$. To compare their work to the results in the present study, a dataset taken from (Horstman et al. 2017b) of the top 1,000 Arabidopsis genes whose upstream promoter sequences were most abundantly bound by BBM were BLASTed to the sorghum proteome, and top hits were reciprocally BLASTed back to Arabidopsis and filtered in order to identify sorghum orthologs and compare them to sets of upregulated and downregulated genes across conditions. 
855 unique sorghum orthologs of BBM ChIP-seq targets were identified. Of these, 110 were present in the downregulated gene set and 112 were present in the upregulated gene set.

$B B M$ can regulate itself by binding to its own promoter sequence and was among the set of $B B M$ targets that were upregulated in this study. The sorghum ortholog of BBM (Sobic.003G390600) was upregulated at the latter four time-points across conditions. Notable sets of $B B M$ targets in the upregulated set included other AP2/ERF transcription factors such as Sobic.004G214300, Sobic.006G245500, Sobic.010G215100 (orthologs of PLETHORA 2 [PLT2], AINTEGUMENTA-like 5 [AIL5], and heat shock complementing factor 1 [HSCF1], respectively), bHLH family transcription factors Sobic.002G255200, Sobic.010G145200, and Sobic.010G172100, and NAC domain transcription factors Sobic.004G112200, Sobic.010G155100, and Sobic.008G094700 (first two genes orthologs of SOMBRERO, latter gene ortholog of VND-INTERACTING2). In addition, two LOB transcription factors Sobic.001G503100 and Sobic.004G348800 (both orthologs of $L B D 16)$, Sobic.004G348800, an ortholog of the $\mathrm{C} 2 \mathrm{H} 2$ zinc finger family member ENHYDROUS, Sobic.002G425600, an ortholog of the bZIP transcription factor ATbZIP12, Sobic.005G132000, an ortholog of ARF2 and Sobic.002G421800, an ortholog of WOX11, were among the BBM targets upregulated across conditions. The auxin efflux carriers Sobic.002G421800, Sobic.010G095100, and Sobic.005G025900 (orthologs of PIN1, PIN1, and PIN4, respectively), Sobic.002G274500, an ortholog of SRS1 which is a 
SHORT INTERNODES-related gene family and a putative abscisic acid transporter, Sobic.005G061000, an ortholog of the ABC transporter family member ABCG25 were also potential targets of $B B M$ that were upregulated in this study.

\section{Discussion}

\section{Assessment of differentially expressed genes by functional category}

Rather than relying on a clustering strategy to organize differentially expressed genes into groups based on similarity in expression curve, the approach taken in this study to group and analyze the large set of differentially expressed genes was to examine the intersection with sets of interesting genes. The interesting sets chosen were curated based on broad functional categories of genes known to be important in the somatic embryogenesis process (i.e., epigenetic modulators, transcription factors, and hormone-related genes), miscellaneous Gene Ontology terms highlighted in gene set enrichment analysis, and gene sets taken from previous studies. This allowed for dissection and manual review of a few focused subsets of differentially regulated genes, some of which may prove to play key regulatory roles in somatic embryogenesis (as opposed to more downstream, minor roles) if investigated further through future research projects.

There were few epigenetic modulator genes found to be differentially regulated in this study relative to the number of predicted genes in the sorghum genome as a whole. There were no apparent downregulated EM genes seen in this study. Among the 
upregulated set of genes were 2 DNA-modifying proteins and 6 putative histone-modifying proteins. The 2 DNA-modifying proteins were Sobic.001G055700, an ortholog of DNA methyltransferase 1 (MET1), which functions in methylation of $\mathrm{CpG}$ targets and Sobic.001G188500, an ortholog of DEMETER (DME), which functions in excision of 5methylcytosine residues. Since these upregulated genes participate in apparently opposite molecular functions, it is possible that they target different sets of genes to control their expression during early embryogenesis. It is also possible that one or both of them do not carry out their molecular function at this stage and that they are incidentally upregulated as part of a general transcriptional program in which only a few genes are actually important for somatic embryogenesis. Further research will be required to understand what roles, if any, these genes play.

The histone-modifying proteins that were upregulated included: 1 histone deacetylase (Sobic.003G396600; an ortholog of HDA3), which serves to decrease the expression of genes in the vicinity of the histone molecules it targets, 2 genes annotated as H3K4me3 demethylases (Sobic.001G284400 and Sobic.004G338500; orthologs of AT5G46910 and PKDM7D, respectively), and 3 genes annotated as H3K9 demethylases (Sobic.001G175000, Sobic.002G193300, and Sobic.004G006600; orthologs of JMJ29, AT1G11950, and AT1G11950, respectively). Based on their annotated functions, these genes all probably play repressive roles in regulating gene expression, though, depending on the degree of methylation present at H3K4 and H3K9 residues, demethylation can lead to either repression or activation. Several EM genes are either known or suspected 
to play a role in repression of tissue dedifferentiation and hence somatic embryo development in plants including the H3K9 methyltransferase KRYPTONITE (KYP), the CHD3 chromatin remodeling factor PICKLE (PKL), and sets of genes that together make up Polycomb Repressive Complexes 1 and 2 (PRC1/2) (Horstman et al. 2017a). Though none of the sorghum orthologs of these genes were found to be differentially regulated in this study, it is interesting that KYP has an opposite molecular function to three of the upregulated histone-modifying genes and thus, one possibility is that these genes work to counter the role of $K Y P$ in repressing dedifferentiation.

This study revealed the upregulation of many transcription factor genes from diverse protein families. The role genes in the AP2/ERF family of transcription factors, in addition to $B B M$, in embryogenesis has been well-established (Horstman et al. 2017b). This, combined with the fact that $B B M$ can directly regulate the expression of its close relatives, makes it unsurprising to see orthologs of $P L T 2$, and AIL5 strongly upregulated in the treatment condition. LOB family transcription factors are also known to promote callus formation (Fan et al. 2012). Thus, it might also be expected that several LOB genes would be expressed upon auxin induction, including the two sorghum orthologs or LBD16 (Sobic.001G503100 and Sobic.004G348800) noted previously. The MADS box transcription factor AGL15 has been shown to enhance somatic embryo development in multiple dicot species (Thakare et al. 2008) and Salvo et al. (2014) reported strong upregulation of an AGL15 ortholog (GRMZM2G139073) across time in tissue culture. The authors of this study hypothesized that $A G L 15$-like genes may play an essential role in maize callus induction and maintenance. However, in the present study, no genes from 
the MADS transcription factor family were differentially expressed, suggesting these genes may not be essential for callus formation in sorghum.

Members of the bHLH and NAC domain-containing families have not been previously identified as those playing a role in somatic embryogenesis, so to see several members of these families strongly upregulated suggests they may have some functional role to play. The bHLH proteins Sobic.001G430000, Sobic.001G488400, Sobic.002G198500, Sobic.002G230000, Sobic.004G237000, Sobic.007G135900, and Sobic.010G051800 are differentially expressed beginning very early in the time-course and they are not evidently under the regulatory control of $B B M$. Thus, it is possible that they represent an earlier transcriptional program than the one induced by $B B M$, or a separate one entirely. The same is true of the NAC family transcription factors Sobic.001G316800, Sobic.001G522700, Sobic.002G259600, Sobic.002G421400, Sobic.004G237900, Sobic.009G231600, and Sobic.010G002900, though two of them (Sobic.004G112200 and Sobic.010G155100; orthologs of SOMBRERO) were among the promoter binding targets of $B B M$. Additional transcription factors that could be good candidates for further investigation into their potential role in somatic embryogenesis include the bZIP transcription factor Sobic.005G041100, the MYB-related transcription factor Sobic.003G129000, and the ABI3VP1 B3 transcription factors Sobic.001G164800 and Sobic.003G281300. It is also interesting that WRKY family transcription factor genes, which are well-known for their involvement in regulating plant stress responses, were overrepresented among downregulated genes very early in tissue culture at the 6-hour time-point. This is too early in time for these genes to be associated with the precocious 
germination the embryo eventually undergoes in the absence of 2,4-D in tissue culture. It is possible that these early downregulated WRKY genes could be actively suppressed by the early auxin response.

It should certainly be expected that the plant hormone most highly represented among the upregulated hormone pathway genes would be auxin since $2,4-D$ is an extremely potent auxin-mimicking compound. The expression of a wide swath of $A U X / I A A$ genes along with $A R F$ transcription factors is indicative of the strong auxin response induced in the treatment condition. Though several IAA family members were not significantly differentially expressed, one apparently anomalous observation was that three members (Sobic.003G035700, Sobic.009G069700, and Sobic.009G203700 (all orthologous to IAA26/PAP1) were consistently downregulated at the latter three time-points. These might represent a kind of negative feedback response to exogenous auxin application that moderates the transcriptional effect. Additionally, auxin biosynthesis genes were not seen among the set of upregulated genes and orthologs of YUC9, YUC1, and TAR2 (Sobic.002G120400, Sobic.003G236900, and Sobic.003G052700, respectively), were actually downregulated across conditions. This is unexpected, as an increase in endogenous auxin is frequently seen in explants competent to undergo somatic embryogenesis (Méndez-Hernández et al. 2019). However, biosynthesis is not the only mechanism for increasing endogenous auxin concentration and genes involved in directional auxin transport and auxin-amino acid conjugate metabolism were seen among the upregulated genes. 
Abscisic acid is also often upregulated in somatic embryogenesis (Jiménez 2001) and genes putatively involved with ABA transport (Sobic.005G061000), signaling (Sobic.001G400800, Sobic.001G400900, and Sobic.003G363400), and response (Sobic.001G446800 and Sobic.006G018400) were seen in the upregulated set. Cytokinin signaling was evidently suppressed in the treatment condition as 5 sorghum genes orthologous to all type-A ARR genes in Arabidopsis were upregulated, although genes corresponding to LOG3 and LOG7, which are involved in cytokinin biosynthesis, were also upregulated. Ethylene is generally understood to have an inhibitory effect on somatic embryogenesis. The suppression of ethylene in the treatment condition is supported by the presence of an ortholog of Arabidopsis RTE1 (Sobic.009G213600), which is a negative regulator of ethylene signaling, among only 3 ethylene-related proteins upregulated at any time-point of the cross-condition comparison. Finally, genes involved in brassinosteroid biosynthesis (Sobic.001G172400), response (Sobic.010G091700 and Sobic.002G221000; orthologs of KANDARI and MALE STERILITY 1, respectively), and signaling (Sobic.001G030400 and Sobic.009G179000) were seen in the set of upregulated genes. The latter signaling-related gene is an ortholog of the rice $B A K 1 / S E R K 1$ and should be investigated for its possible involvement in promoting somatic embryogenesis through cellular signaling pathways.

\section{Comparison to findings of similar studies}

Salvo et al. (2014) investigated gene expression changes over time at 0, 24, 36, 48, and 72 hours after tissue culture initiation on 2,4-D containing media (no 2,4-D-free 
control was used). The researchers reported significant expression changes ( $>1,500$-fold from $0 \mathrm{~h}$ to $24 \mathrm{~h}$ ) in the maize orthologs of the genes WOUND-INDUCED PROTEIN 1 (WIP1; GRMZM2G156632) and chitinase A1 (GRMZM2G051943). However, sorghum orthologs and close relatives of these genes, Sobic.003G085300, Sobic.003G085400, Sobic.003G088400 and Sobic.003G156000, Sobic.006G132100, Sobic.006G132200, Sobic.006G132300, Sobic.006G132400, respectively, did not show such drastic changes in expression within the same condition over time and or any significant differential expression across time, with the exception of Sobic.003G088400, which displayed a moderate degree of upregulation in contrasts at the last three time-points. Though it is possible this difference in results between the two studies could reflect a true difference in the auxin response between maize and sorghum, the use of a control condition in our experiment provided a way to differentiate genes truly induced by 2,4-D exposure from those only induced through tissue culture response.

Du et al. (2019) conducted a study similar in design to this one in the inbred maize line CAL. They collected tissue derived from embryo explant culture at $0,1,2,4$, 6 , and 8 days following initiation in culture and, as in this study, using a parallel control condition with media lacking 2,4-D at each time-point. They reported upregulation in the treatment condition of $65 \mathrm{bHLH}$ factor genes, 46 members of the AP2/ERF family, 39 MYB-related transcription factors, 36 WRKY transcription factors, 31 NAC domain-containing proteins, 18 ARF transcription factors, and 13 LOB domain factors. Taking into account the variations in transcription factor gene copy number between maize and sorghum, this was largely in line with what was observed in this study with the exception of 
MYB and WRKY transcription factors, for which far fewer members of each family were upregulated across conditions in sorghum than in maize. The authors also reported differential regulation of maize orthologs of $C O I 1, J A Z$, and $M Y C 2$, a group of genes involved in jasmonic acid signaling. However, in the present study we found no comparable expression changes with respect to orthologs of these genes in sorghum.

Potential improvements to study design

A flaw in the implementation of this study was the fact that the 0-hour timepoint had to be excluded from analysis, which diminished the usefulness of the acrosstime differential expression contrasts. Initially, for the 0-hour samples immature embryo explants were isolated from sorghum seeds and immediately transferred to a collection tube and flash-frozen for later processing. However, after processing, RNA from 0-hour samples failed to pass quality control checks in contrast to all other samples. This was thought to be due to the large amount of starchy endosperm coating the isolated embryos interfering with the RNA extraction process. Thus, in future sample collections, embryos collected for the 0 -hour time-point only were washed 3 times in sterile deionized water before being blotted dry and flash frozen for later processing. This enabled the extracted RNA to pass quality checking, but after RNA-sequencing it became evident that the 0-hour time-point samples, though highly correlated with each other, were highly deviated from all other collected samples. Cleaning the quality trimmed reads of those aligning to sorghum mitochondrial and ribosomal DNA sequences resulted in the 
removal of more than half of all reads from each sample (see Table 2.1), possibly indicating a strong stress response involving the mass expression of mitochondrial genes. A multi-dimensional scaling plot of all samples showed large distances between the Ohtimes and all other samples (see Figure 2.2A). In retrospect, the appropriate technical procedure to correct for liquid endosperm contamination would have been to wash all immature embryo samples at every time-point with the buffered liquid inoculation medium that is used in the sorghum transformation process prior to tissue collection or placement into tissue culture.

One problem encountered in profiling differential gene expression in early embryogenesis/callus formation is that over time, different cells within the explant tissue/callus have varying responses and only relatively few cells within the tissue as a whole will become progenitors of somatic embryos. Thus, particularly at the latter stages of callus formation, the signal of differentially expressed genes in proto-embryonic cells or cell clusters will be diluted by processing of the whole explant or callus tissue. This could be a cause, for instance, of not detecting the sorghum ortholog of WUS as a differentially expressed gene in this study due to low abundance, as WUS is likely only expressed in very few cells at such early time-points in the embryogenesis process. Therefore, the use of methods that attempt to isolate cells expressing totipotency or embryogenic commitment, perhaps through the use of visually detectable markers for these attributes, would be highly beneficial in future studies for capturing important gene expression dynamics. (Magnani et al. 2017) utilized a method called INTACT (isola- 
tion of nuclei tagged in specific cell types) to separate out nuclei of cells within Arabidopsis callus tissue undergoing somatic embryogenesis prior to profiling with RNA-seq to compare embryogenic cells to non-embryogenic proliferating callus. Another technique that might be applied here to isolate embryogenic cells within callus tissue is manipulation by laser-capture microdissection (Nakazono et al. 2003).

There are experimental parameters that could be applied in future differential expression studies on somatic embryogenesis in order to more narrowly apprehend genes that are key regulators of the process. For instance, the efficiency of tissue culture regeneration procedures is often dependent on a particular range of concentrations of the plant growth regulator being used in callus induction medium. Adding experimental treatments where the 2,4-D concentration is varied to a high enough degree to cause varying regeneration responses could be a way to identify which genes are not only responsive to auxin, but are more highly expressed in conditions leading to efficient regeneration. Another way of probing this question would be to vary the type of auxin used in callus induction media (e.g., IAA, NAA) in addition to the concentration.

Another possibility is that genotype strongly influences either the expression of genes necessary for somatic embryogenesis in response to auxin or that, in some genomic contexts, auxin leads to the induction (or repression) of a module of genes that works to curb regeneration through somatic embryogenesis. To investigate this, an experiment that utilizes multiple different genotypes, ones considered highly-regenerable 
versus ones considered only moderately regenerable or recalcitrant, could be highly informative for identifying genotype-dependent transcriptional responses that influence the capacity for regeneration through somatic embryogenesis.

Since what was actually being measured in this study (as a proxy for differential gene expression in embryogenic versus non-embryogenic explant tissue) was whole explant transcriptional response to 2,4-D through time, it is probable that many of the differentially expressed genes highlighted here, and in other similar studies, are part of a generalized response to exogenous auxin application in the immature zygotic embryo tissue context which have no important role to play in the somatic embryogenic pathway. Noting that the sorghum ortholog of the $B B M$ gene was not significantly upregulated in contrast to the control condition until the final three time-points, an interesting experiment to perform would be to compare transcriptional profiles of explant tissue undergoing either 2,4-D or transgenic BBM-induced embryogenesis at a few early timepoints. This might make it possible to help tease apart whether, for instance, some of the transcription factor genes noted here such as the NAC and bHLH family members which were apparently induced earlier in time than $B B M$ are actually important in somatic embryogenesis, or are only incidentally induced by 2,4-D application.

\section{Limitations of RNA-seq}

RNA-seq itself has multiple limitations as a tool for identifying important genes involved in a complex developmental processes such as somatic embryogenesis. Aside from the fact that it often implicates a very large number of differentially expressed 
genes that can be difficult and time-consuming to sift through and single out individual genes for hypothesis testing, RNA-seq may not even capture all genes that play an essential role in the process. Since RNA-seq relies on differences in mRNA expression of genes that are detectable above a certain threshold, a gene involved for instance, in signal transduction leading to somatic embryogenesis, will not be detected by RNA-seq if there is no downstream feedback that results in differential regulation of its own transcription. It is possible that this is the case with the SERK genes, where it has been established that their ectopic expression can significantly increase embryogenic competence (Hecht et al. 2001, Hu et al. 2005, Salaj et al. 2008). As mentioned earlier, certain chromatin modifying genes that are known to inhibit dedifferentiation and embryogenesis by epigenetically repressing the expression of certain embryogenesis-regulating factors, would not be detectable by RNA-seq if the mechanism of de-repression does not involve these genes. The fact that changes to the expression of genes orthologous to $K Y P, P K L$, and the components of PRC1 and PRC2 were not seen in this study supports that this might be the case.

Furthermore, RNA-seq studies tend to emphasize genes that display large expression fold-changes between samples when these are not necessarily likely to be the most indispensable genes in governing a developmental process. Due to the nature of transcriptional regulatory networks, relatively small changes to the expression of key genes can lead to much more dramatic changes in the expression of hundreds or even thousands of genes that may be separated from them by multiple degrees of interaction. Thus, it is possible for an arbitrary fold-change cut-off to exclude these key genes 
from being highlighted by differential expression analysis if they show only modest augmentation of expression levels between conditions. RNA-seq can be useful for correlating genes by their expression curves or using clustering algorithms to highlight sets of genes that may be referred to as "co-expressed." However, RNA-seq data alone cannot be used to establish a common regulatory interaction between genes or to determine a regulatory hierarchy. One other weakness of RNA-seq is that it uses gene transcription as a proxy for establishing expression of a functional gene product, though these are not necessarily correlated in every case. This excludes the influence of post-transcriptional and post-translational processes that can significantly alter the expression of the final gene product. One such process known to have some control over somatic embryogenesis is miRNA-mediated gene silencing (Siddiqui et al. 2019), of which the influence on the expression of individual genes will not be accurately reflected by RNA-seq analysis.

\section{Conclusion and future perspectives}

There remains very little understanding of how the somatic embryogenesis process is initiated on a genomic level. One fact that is well-established at this point is that the $A P 2$-like transcription factor $B B M$ plays a central role in governing this process. It is also established that $B B M$ can either directly or indirectly regulate the expression of several other genes that have at least some influence over somatic embryogenesis. But what is not understood is the sequence and setting of events surrounding $B B M$ expression. Some important questions to be addressed in future research might include the 
following: What series of molecular interactions occurs that results in the induction of $B B M$ from exogenous auxin application? Is $B B M$ only induced in particular cells or cell types? If so, what genomic/epigenomic states prime such cells for $B B M$ induction? Is there and relationship between $B B M$ and the SERK signaling genes? Is there any regulatory cascade connecting $B B M$ to the expression of WUS in proto-meristematic regions? To what extent can related AP2-like transcription factors such PLT2 and AlL5 compensate for regulating the embryogenic program initiated by $B B M$ ? Is there a BBM/PLT2/AIL5-independent somatic embryogenesis pathway?

This study found the transcript of the sorghum $B B M$ ortholog to be first differentially regulated at 24 hours following 2,4-D induction. Since many genes (particularly transcription factor members of the bHLH and NAC domain-containing families) were strongly upregulated prior to this, it would be interesting to investigate whether any of these genes either: A) play a role in establishing an environment where $B B M$ is able to regulate somatic embryogenesis (i.e., involvement in cellular dedifferentiation prior to embryogenic commitment); or B) precede BBM induction in a regulatory hierarchy either directly or indirectly. It is also notable that several LOB domain factors were apparently induced prior to $B B M$. This, in a sense, is not completely surprising as the genes ARF7 and ARF19 are known to directly activate $L B D 16$ and $L B D 29$ in Arabidopsis lateral root formation (Okushima et al. 2007), the sorghum orthologs of which were all upregulated in this study. However, $B B M$ can also bind the promoter regions of these genes (Horstman et al. 2017b), so it is unclear which potential mode of regulation might be more important in the somatic embryogenesis process. ChIP-seq experiments using 
transcription factor sequences such as $L B D 16$ or $L B D 29$ as probes will assist in identifying what genes among those upregulated during somatic embryogenesis might be directly regulated by these factors. To identify factors that might be directly responsible for the induction of $B B M$, a reverse ChIP technique (Wen et al. 2020) could be used to capture proteins binding the $B B M$ promoter and determine their identity by mass spectrometry. Finally, bisulfite sequencing experiments, possibly in combination with ChIPseq targeting different histone covalent modifications, could provide valuable insight into how epigenetic regulation influences somatic embryogenesis competence in different genetic backgrounds or treatments.

\section{Materials and methods}

Plant materials

Sorghum plant used as a source for immature embryos in this study were grown from seed in a greenhouse on the University of Missouri campus in Columbia, MO in the fall of 2015 following the protocol described by Do et al. (2016). After sorghum seed heads emerged, they were each covered with a tassel bag to promote self-pollination. 10-13 days following pollination, immature seeds were collected for placement into tissue culture.

\section{Tissue culture media}

The media used for tissue culture experiments consisted of an amended formulation of the sorghum callus induction medium (CIM) described in Do et al. (2016). The 
recipe for this media is as follows: $4.3 \mathrm{~g} / \mathrm{L} \mathrm{MS}$ salts (Murashige and Skoog 1962), $0.5 \mathrm{~g} / \mathrm{L}$ 2-(4-morpholino) ethane sulfonic acid (MES), $1.0 \mathrm{~g} / \mathrm{L}$ L-proline, $1.0 \mathrm{~g} / \mathrm{L}$ L-asparagine, 1.0 $\mathrm{g} / \mathrm{L}$ monobasic potassium phosphate $\left(\mathrm{KH}_{2} \mathrm{PO}_{4}\right), 1.5 \mathrm{mg} / \mathrm{L}$ 2,4-dichlorophenoxyacetic acid (2,4-D), $30 \mathrm{~g} / \mathrm{L}$ sucrose, $8 \mathrm{~g} / \mathrm{L}$ washed agar, and $10 \mathrm{~g} / \mathrm{L}$ Polyvinylpolypyrrolidone (PVPP) were added to deionized water, which was then $\mathrm{pH}$ adjusted to 5.8 using a $1 \mathrm{M}$ solution of potassium hydroxide (KOH). The media was then autoclaved for 30 minutes and a filter-sterilized mixture of 100X B5 vitamins (Gamborg et al. 1968) and $0.16 \mathrm{mg} / \mathrm{L}$ cupric sulfate $\left(\mathrm{CuSO}_{4}\right)$ was added post-autoclaving as it cooled. Media was poured into $100 \mathrm{x}$ $15 \mathrm{~mm}$ petri plates. For the control condition (referred to as 2,4-D [-]), an amended media was made that omitted 2,4-D as an ingredient but was otherwise formulated as described above.

\section{Embryo tissue culture initiation and collection}

Immature sorghum seeds harvested from greenhouse plants were removed individually from panicles and then sterilized in a 50\% bleach solution for approximately 15 minutes before being rinsed 3-4 times with sterile deionized water. In sterile conditions under a laminar flow hood, immature embryos were isolated from seeds using a \#15 scalpel blade. Embryos were placed directly (scutellum-side up) onto petri plates containing CIM or CIM [-], with the exception of the 0-hour time samples, which were rinsed 3 times in sterile deionized water to remove the starchy endosperm, dried with a 
Kimwipe, transferred to a $1.7 \mathrm{~mL}$ Eppendorf tube, and then immediately preserved by flash freezing in liquid nitrogen. Other samples were collected from tissue culture in an Eppendorf tube and flash-frozen at their designated time-points. About 25 individual immature embryos were collected for each sample. This experiment was repeated in triplicate other the course of about 1 month using different greenhouse-grown sorghum plants as donors of regenerative tissue for each replicate. All frozen samples were stored at $-80^{\circ} \mathrm{C}$ for up to two weeks until they could be processed.

Sample processing and transcriptome sequencing

Total RNA was extracted using the TRIzol reagent and following the associated protocol (Invitrogen, http://www.invitrogen.com). RNA integrity was assessed by agarose gel electrophoresis and was quantified and assessed for DNA or protein contamination using a NanoDrop. Approximately $5 \mu \mathrm{g}$ of total RNA from each sample was submitted for mRNA processing, cDNA library construction and sequencing to the University of Missouri DNA Core Facility. The RNA libraries were fragmented into 51-nucleotide shortread sequences, converted to cDNA, PCR-amplified using the Illumina TruSeq (v3) RNA sample preparation protocol and sequenced using the Illumina HiSeq 2500 platform. Individual samples were split over three separate runs. The first run was carried out in paired-end mode while the other two runs used single-end reads. Raw read data was delivered in FASTQ format following quality control analysis.

Data processing and gene expression quantification 
Raw RNA-seq reads were quality trimmed to remove adapter sequences and low-quality regions using Trimmomatic version-0.35 (Bolger et al. 2014) with the "Sliding window" method and discarding reads trimmed shorted than 36 bases in length. The sets were then "cleaned" of sorghum mitochondrial DNA and ribosomal DNA sequences, which were acquired from the NCBI genome database and Silva (Quast et al. 2012), respectively, by reverse aligning the trimmed reads to a custom database containing these sequences using Bowtie2 (Langmead et al. 2019) with the --un option. Trimmed and cleaned reads were then aligned to the Sorghum bicolor v3.1.1 reference genome (McCormick et al. 2018) downloaded from Phytozome v12 (accession ID.: ABXC03000000) using HISAT2 (Kim et al. 2015) with the --dta tag option enabled to combine all files generated over multiple sequencing runs corresponding to the same biological sample. StringTie (Pertea et al. 2015) was used to assemble alignments into transcripts using the GFF3 annotation file associated with the v3.1.1 genome build as a guide. To identify putative novel genes and transcripts not annotated in the reference, all assembled transcripts not contained in the reference were extracted from each sample's annotation GTF file and then merged into a single annotation using StringTie with the --merge option invoked. Gffcompare (Pertea and Pertea 2020) was used to identify transcripts that were essentially matches or sequence-overlapping between the reference annotation and novel transcript set. These matches were removed, leaving behind only those novel transcripts spanning genomic regions where genes had not previously been annotated. These novel annotations were then combined with the reference annotation file and this new file was used as a guide to re-run transcript assembly with 
StringTie. FPKM values for genes from each sample were extracted from the StringTie output and used to construct a data table from which a Pearson correlation matrix was generated using the "corr" function in R. Per-gene read counts were also generated for each sample using featureCounts (Liao et al. 2014) and were combined into a single read count data set in preparation for differential expression analysis.

\section{Differential expression analysis}

The EdgeR package (Robinson et al. 2010) was used to perform statistical analysis of differentially expressed genes. Samples were grouped by biological replicates time-point and experimental condition. Only genes with read count values greater than or equal to 5 in at least two samples were retained for further analysis. Values were then normalized across samples. To visualize the expression variance between samples, plotting by multidimensional scaling was employed using the "plotMDS" function of the EdgeR package. The design matrix employed comparisons both across time with each successive time-point in each condition being contrasted with the first time-point (i.e., 6-hours) in the same condition, and across conditions with each time-point in the treatment condition being contrasted with the corresponding time in the control condition. A generalized linear model was employed with the "glmFit" function of EdgeR and a likelihood ratio test was used for gene level comparisions and p-values from these tests were type I error-corrected using the Benjamini-Hochberg procedure to yield False Discovery Rates (FDR) for each gene-wise test. Genes with at least a 2-fold expression change and an FDR value below 0.05 for a given contrast were considered differentially expressed. 
Gene set enrichment analysis

Sets of ID tags for differentially expressed genes in each contrast were uploaded to the Sorghum Functional Genomics Database (http://structuralbiology.cau.edu.cn/sorghum/index.html) (Tian et al. 2016) GSEA webpage tool and were tested for functional category enrichment against a whole sorghum genome background. The functional annotation sets chosen were Gene Ontology biological process and molecular function categories, along with Gene Family (Gfam) sets and PlantCyc and KEGG pathway sets. Enrichment was assessed using Fisher's exact test with the Yekutieli multi-test adjustment method. Functional sets with an adjusted $P$-value less than 0.05 were considered significantly enriched.

Gene annotation through orthology

Primary translation products of the set of all differentially expressed sorghum genes were searched against whole proteome databases of Zea mays, Oryza sativa, and Arabidopsis thaliana with blastp via the BLAST+ software package (Camacho et al. 2009). The top 10 BLAST hits (with an E-value cutoff of 1 x E-5) from each proteome were retained and for hits associated with multiple genes, the ortholog was assigned to the sorghum gene for which it had the lowest E-value. For identifying genes likely to be involved in regulation of gene expression including transcription factors and epigenetic modulators, multiple alignments for the definitive domains of each of these were downloaded from the PInTFDB website (http://plntfdb.bio.uni-potsdam.de/v3.0/) (Pérez- 
Rodríguez et al. 2010) and were used to build Hidden Markov Models and scan the set of differentially expressed sorghum peptide sequences for them using the HMMer software package (Eddy 2011). Protein sequences of genes found to be important in the zygotic or somatic embryogenesis process in prior studies were also BLASTed against the sorghum proteome to identify their putative orthologs. 


\section{Reference List}

Abou-Elwafa SF, Amin AE-EAZ, Shehzad T. 2019. Genetic mapping and transcriptional profiling of phytoremediation and heavy metals responsive genes in sorghum. Ecotoxicology and environmental safety 173: 366-372.

Altpeter F, Springer NM, Bartley LE, Blechl AE, Brutnell TP, Citovsky V, Conrad L, Gelvin SB, Jackson DP, Kausch AP. 2016. Advancing crop transformation in the era of genome editing. The Plant Cell 28: 1510-1520.

Binns D, Dimmer E, Huntley R, Barrell D, O'donovan C, Apweiler R. 2009. QuickGO: a web-based tool for Gene Ontology searching. Bioinformatics 25: 3045-3046.

Bolger AM, Lohse M, Usadel B. 2014. Trimmomatic: a flexible trimmer for Illumina sequence data. Bioinformatics 30: 2114-2120.

Boutilier K, Offringa R, Sharma VK, Kieft H, Ouellet T, Zhang L, Hattori J, Liu C-M, van Lammeren AA, Miki BL. 2002. Ectopic expression of BABY BOOM triggers a conversion from vegetative to embryonic growth. The Plant Cell 14: 1737-1749.

Bouyer D, Roudier F, Heese M, Andersen ED, Gey D, Nowack MK, Goodrich J, Renou J-P, Grini PE, Colot V. 2011. Polycomb repressive complex 2 controls the embryo-toseedling phase transition. PLoS Genet 7: e1002014.

Camacho C, Coulouris G, Avagyan V, Ma N, Papadopoulos J, Bealer K, Madden TL. 2009. BLAST+: architecture and applications. BMC bioinformatics 10: 1-9.

Chandrashekar A, Satyanarayana K. 2006. Disease and pest resistance in grains of sorghum and millets. Journal of Cereal Science 44: 287-304.

Che P, Anand A, Wu E, Sander JD, Simon MK, Zhu W, Sigmund AL, Zastrow-Hayes G, Miller M, Liu D. 2018. Developing a flexible, high-efficiency Agrobacteriummediated sorghum transformation system with broad application. Plant biotechnology journal 16: 1388-1395.

Chen Y, Xu X, Liu Z, Zhang Z, XuHan X, Lin Y, Lai Z. 2020. Global scale transcriptome analysis reveals differentially expressed genes involve in early somatic embryogenesis in Dimocarpus longan Lour. BMC genomics 21: 1-22.

de Carvalho Silva R, Carmo LST, Luis ZG, Silva LP, Scherwinski-Pereira JE, Mehta A. 2014. Proteomic identification of differentially expressed proteins during the 
acquisition of somatic embryogenesis in oil palm (Elaeis guineensis Jacq.). Journal of proteomics 104: 112-127.

de Oliveira AA, Pastina MM, da Costa Parrella RA, Noda RW, Simeone MLF, Schaffert RE, de Magalhães JV, Damasceno CMB, Margarido GRA. 2018. Genomic prediction applied to high-biomass sorghum for bioenergy production. Molecular Breeding 38: 1-16.

Do PT, Lee H, Mookkan M, Folk WR, Zhang ZJ. 2016. Rapid and efficient Agrobacteriummediated transformation of sorghum (Sorghum bicolor) employing standard binary vectors and bar gene as a selectable marker. Plant cell reports 35: 20652076.

Du X, Fang T, Liu Y, Huang L, Zang M, Wang G, Liu Y, Fu J. 2019. Transcriptome profiling predicts new genes to promote maize callus formation and transformation. Frontiers in plant science 10: 1633.

Eddy SR. 2011. Accelerated profile HMM searches. PLoS Comput Biol 7: e1002195.

Eggen M, Ozdogan M, Zaitchik B, Ademe D, Foltz J, Simane B. 2019. Vulnerability of sorghum production to extreme, sub-seasonal weather under climate change. Environmental Research Letters 14: 045005.

Elhiti M, Stasolla C, Wang A. 2013. Molecular regulation of plant somatic embryogenesis. In Vitro Cellular \& Developmental Biology-Plant 49: 631-642.

Fan M, Xu C, Xu K, Hu Y. 2012. LATERAL ORGAN BOUNDARIES DOMAIN transcription factors direct callus formation in Arabidopsis regeneration. Cell research 22: 1169-1180.

Fehér A. 2015. Somatic embryogenesis - stress-induced remodeling of plant cell fate. Biochimica et Biophysica Acta (BBA)-Gene Regulatory Mechanisms 1849: 385402.

Fehér A. 2019. Callus, dedifferentiation, totipotency, somatic embryogenesis: what these terms mean in the era of molecular plant biology? Frontiers in plant science 10: 536.

Gao L, Zhang J, Hou Y, Yao Y, Ji Q. 2015. RNA-Seq screening of differentially-expressed genes during somatic embryogenesis in Fragaria $\times$ ananassa Duch. 'Benihopp'. The Journal of Horticultural Science and Biotechnology 90: 671-681. 
Gordon-Kamm B, Sardesai N, Arling M, Lowe K, Hoerster G, Betts S, Jones T. 2019. Using morphogenic genes to improve recovery and regeneration of transgenic plants. Plants 8: 38.

Guo F, Liu C, Xia H, Bi Y, Zhao C, Zhao S, Hou L, Li F, Wang X. 2013. Induced expression of AtLEC1 and AtLEC2 differentially promotes somatic embryogenesis in transgenic tobacco plants. PLoS One 8: e71714.

Hecht V, Vielle-Calzada J-P, Hartog MV, Schmidt ED, Boutilier K, Grossniklaus U, de Vries SC. 2001. The Arabidopsis SOMATIC EMBRYOGENESIS RECEPTOR KINASE 1 gene is expressed in developing ovules and embryos and enhances embryogenic competence in culture. Plant Physiology 127: 803-816.

Heidmann I, De Lange B, Lambalk J, Angenent GC, Boutilier K. 2011. Efficient sweet pepper transformation mediated by the $B A B Y B O O M$ transcription factor. Plant cell reports 30: 1107-1115.

Horstman A, Bemer M, Boutilier K. 2017a. A transcriptional view on somatic embryogenesis. Regeneration 4: 201-216.

Horstman A, Li M, Heidmann I, Weemen M, Chen B, Muino JM, Angenent GC, Boutilier K. 2017b. The BABY BOOM transcription factor activates the LEC1-ABI3-FUS3LEC2 network to induce somatic embryogenesis. Plant physiology $175: 848-857$.

Howe A, Sato S, Dweikat I, Fromm M, Clemente T. 2006. Rapid and reproducible Agrobacterium-mediated transformation of sorghum. Plant cell reports 25: 784791.

$\mathrm{Hu} \mathrm{H}$, Xiong L, Yang Y. 2005. Rice SERK1 gene positively regulates somatic embryogenesis of cultured cell and host defense response against fungal infection. Planta 222: 107-117.

Huang S, Hill RD, Wally OS, Dionisio G, Ayele BT, Jami SK, Stasolla C. 2014. Hemoglobin control of cell survival/death decision regulates in vitro plant embryogenesis. Plant physiology 165: 810-825.

Kausch AP, Nelson-Vasilchik K, Hague J, Mookkan M, Quemada H, Dellaporta S, Fragoso C, Zhang ZJ. 2019. Edit at will: Genotype independent plant transformation in the era of advanced genomics and genome editing. Plant Science 281: 186-205. 
Khan MA, Abbasi BH, Ali H, Ali M, Adil M, Hussain I. 2015. Temporal variations in metabolite profiles at different growth phases during somatic embryogenesis of Silybum marianum L. Plant Cell, Tissue and Organ Culture (PCTOC) 120: 127-139.

Kim D, Langmead B, Salzberg SL. 2015. HISAT: a fast spliced aligner with low memory requirements. Nature methods 12: 357-360.

Kim J, Kim J-S. 2016. Bypassing GMO regulations with CRISPR gene editing. Nature biotechnology 34: 1014-1015.

Kumar A, Palni LMS, Sood A, Sharma M, Palni UT, Gupta AK. 2002. Heat-shock induced somatic embryogenesis in callus cultures of gladiolus in the presence of high sucrose. The Journal of Horticultural Science and Biotechnology 77: 73-78.

Langmead B, Wilks C, Antonescu V, Charles R. 2019. Scaling read aligners to hundreds of threads on general-purpose processors. Bioinformatics 35: 421-432.

Liang H, Xiong Y, Guo B, Yan H, Jian S, Ren H, Zhang X, Li Y, Zeng S, Wu K. 2020. Shoot organogenesis and somatic embryogenesis from leaf and root explants of Scaevola sericea. Scientific Reports 10: 1-11.

Liao Y, Smyth GK, Shi W. 2014. featureCounts: an efficient general purpose program for assigning sequence reads to genomic features. Bioinformatics 30: 923-930.

Liu G, Godwin ID. 2012. Highly efficient sorghum transformation. Plant cell reports 31: 999-1007.

Lowe K, La Rota M, Hoerster G, Hastings C, Wang N, Chamberlin M, Wu E, Jones T, Gordon-Kamm W. 2018. Rapid genotype “independent" Zea mays L. (maize) transformation via direct somatic embryogenesis. In Vitro Cellular \& Developmental Biology-Plant 54: 240-252.

Lowe K, Wu E, Wang N, Hoerster G, Hastings C, Cho M-J, Scelonge C, Lenderts B, Chamberlin M, Cushatt J. 2016. Morphogenic regulators Baby boom and Wuschel improve monocot transformation. The Plant Cell 28: 1998-2015.

Magnani E, Jiménez-Gómez J, Soubigou-Taconnat L, Lepiniec L, Fiume E. 2017. Profiling the onset of somatic embryogenesis in Arabidopsis. BMC genomics 18: 1-12. 
Mathieu M, Lelu-Walter M-A, Blervacq A-S, David H, Hawkins S, Neutelings G. 2006. Germin-like genes are expressed during somatic embryogenesis and early development of conifers. Plant molecular biology 61: 615-627.

McCormick RF, Truong SK, Sreedasyam A, Jenkins J, Shu S, Sims D, Kennedy M, Amirebrahimi M, Weers BD, McKinley B. 2018. The Sorghum bicolor reference genome: improved assembly, gene annotations, a transcriptome atlas, and signatures of genome organization. The Plant Journal 93: 338-354.

Méndez-Hernández HA, Ledezma-Rodríguez M, Avilez-Montalvo RN, Juárez-Gómez YL, Skeete A, Avilez-Montalvo J, De-la-Peña C, Loyola-Vargas VM. 2019. Signaling overview of plant somatic embryogenesis. Frontiers in plant science 10: 77.

Mookkan M, Nelson-Vasilchik K, Hague J, Zhang ZJ, Kausch AP. 2017. Selectable marker independent transformation of recalcitrant maize inbred $B 73$ and sorghum P898012 mediated by morphogenic regulators BABY BOOM and WUSCHEL2. Plant cell reports 36: 1477-1491.

Nakazono M, Qiu F, Borsuk LA, Schnable PS. 2003. Laser-capture microdissection, a tool for the global analysis of gene expression in specific plant cell types: identification of genes expressed differentially in epidermal cells or vascular tissues of maize. The Plant Cell 15: 583-596.

Newman Y, Erickson J, Vermerris W, Wright D. 2010. Forage sorghum (Sorghum bicolor): overview and management. EDIS 2010.

Nguyen T-V, Thu TT, Claeys M, Angenon G. 2007. Agrobacterium-mediated transformation of sorghum (Sorghum bicolor (L.) Moench) using an improved in vitro regeneration system. Plant cell, tissue and organ culture 91: 155-164.

Okushima Y, Fukaki H, Onoda M, Theologis A, Tasaka M. 2007. ARF7 and ARF19 regulate lateral root formation via direct activation of $L B D / A S L$ genes in Arabidopsis. The Plant Cell 19: 118-130.

Pérez-Rodríguez P, Riano-Pachon DM, Corrêa LGG, Rensing SA, Kersten B, MuellerRoeber B. 2010. PInTFDB: updated content and new features of the plant transcription factor database. Nucleic acids research 38: D822-D827.

Pertea G, Pertea M. 2020. GFF utilities: GffRead and GffCompare. F1000Research 9. 
Pertea M, Pertea GM, Antonescu CM, Chang T-C, Mendell JT, Salzberg SL. 2015. StringTie enables improved reconstruction of a transcriptome from RNA-seq reads. Nature biotechnology 33: 290-295.

Quast C, Pruesse E, Yilmaz P, Gerken J, Schweer T, Yarza P, Peplies J, Glöckner FO. 2012. The SILVA ribosomal RNA gene database project: improved data processing and web-based tools. Nucleic acids research 41: D590-D596.

Quintana-Escobar AO, Nic-Can GI, Avalos RMG, Loyola-Vargas VM, Gongora-Castillo E. 2019. Transcriptome analysis of the induction of somatic embryogenesis in Coffea canephora and the participation of ARF and Aux/IAA genes. PeerJ 7: e7752.

Rajesh M, Fayas T, Naganeeswaran S, Rachana K, Bhavyashree U, Sajini K, Karun A. 2016. De novo assembly and characterization of global transcriptome of coconut palm (Cocos nucifera L.) embryogenic calli using Illumina paired-end sequencing. Protoplasma 253: 913-928.

Repellin A, Båga M, Jauhar PP, Chibbar RN. 2001. Genetic enrichment of cereal crops via alien gene transfer: new challenges. Plant cell, tissue and organ culture 64: 159183.

Robinson MD, McCarthy DJ, Smyth GK. 2010. edgeR: a Bioconductor package for differential expression analysis of digital gene expression data. Bioinformatics 26: 139-140.

Salaj J, von Recklinghausen IR, Hecht V, de Vries SC, Schel JH, van Lammeren AA. 2008. AtSERK1 expression precedes and coincides with early somatic embryogenesis in Arabidopsis thaliana. Plant Physiology and Biochemistry 46: 709-714.

Salvo SA, Hirsch CN, Buell CR, Kaeppler SM, Kaeppler HF. 2014. Whole transcriptome profiling of maize during early somatic embryogenesis reveals altered expression of stress factors and embryogenesis-related genes. PLoS One 9: e111407.

Santarem ER, Pelissier B, Finer JJ. 1997. Effect of explant orientation, pH, solidifying agent and wounding on initiation of soybean somatic embryos. In Vitro Cellular \& Developmental Biology-Plant 33: 13-19.

Satish K, Srinivas G, Madhusudhana R, Padmaja P, Reddy RN, Mohan SM, Seetharama N. 2009. Identification of quantitative trait loci for resistance to shoot fly in 
sorghum [Sorghum bicolor (L.) Moench]. Theoretical and applied genetics 119: 1425-1439.

Siddiqui ZH, Abbas ZK, Ansari MW, Khan MN. 2019. The role of miRNA in somatic embryogenesis. Genomics 111: 1026-1033.

Silva HG, Sobral RS, Magalhães AP, Morais-Cecílio L, Costa MMR. 2020. Genome-wide identification of epigenetic regulators in Quercus suber L. International journal of molecular sciences 21: 3783.

Smedley D, Haider S, Durinck S, Pandini L, Provero P, Allen J, Arnaiz O, Awedh MH, Baldock R, Barbiera G. 2015. The BioMart community portal: an innovative alternative to large, centralized data repositories. Nucleic acids research 43: W589-W598.

Stone SL, Kwong LW, Yee KM, Pelletier J, Lepiniec L, Fischer RL, Goldberg RB, Harada JJ. 2001. LEAFY COTYLEDON2 encodes a B3 domain transcription factor that induces embryo development. Proceedings of the National Academy of Sciences 98: 11806-11811.

Tanaka M, Kikuchi A, Kamada H. 2008. The Arabidopsis histone deacetylases HDA6 and HDA19 contribute to the repression of embryonic properties after germination. Plant physiology 146: 149-161.

Thakare D, Tang W, Hill K, Perry SE. 2008. The MADS-domain transcriptional regulator AGAMOUS-LIKE15 promotes somatic embryo development in Arabidopsis and soybean. Plant physiology 146: 1663-1672.

Tian T, You Q, Zhang L, Yi X, Yan H, Xu W, Su Z. 2016. SorghumFDB: sorghum functional genomics database with multidimensional network analysis. Database 2016.

Upadhyaya HD, Vetriventhan M, Asiri AM, CR Azevedo V, Sharma HC, Sharma R, Sharma SP, Wang Y-H. 2019. Multi-Trait diverse germplasm sources from mini core collection for sorghum improvement. Agriculture 9: 121.

Wang F-X, Shang G-D, Wu L-Y, Xu Z-G, Zhao X-Y, Wang J-W. 2020. Chromatin accessibility dynamics and a hierarchical transcriptional regulatory network structure for plant somatic embryogenesis. Developmental Cell 54: 742-757. e748. 
Wen X, Wang J, Zhang D, Ding Y, Ji X, Tan Z, Wang Y. 2020. Reverse Chromatin Immunoprecipitation (R-ChIP) enables investigation of the upstream regulators of plant genes. Communications biology 3: 1-12.

Wu E, Lenderts B, Glassman K, Berezowska-Kaniewska M, Christensen H, Asmus T, Zhen S, Chu U, Cho M-J, Zhao Z-Y. 2014a. Optimized Agrobacterium-mediated sorghum transformation protocol and molecular data of transgenic sorghum plants. In Vitro Cellular \& Developmental Biology-Plant 50: 9-18.

Yang X, Zhang X. 2010. Regulation of somatic embryogenesis in higher plants. Critical Reviews in Plant Science 29: 36-57.

Yang X, Zhang X, Yuan D, Jin F, Zhang Y, Xu J. 2012. Transcript profiling reveals complex auxin signalling pathway and transcription regulation involved in dedifferentiation and redifferentiation during somatic embryogenesis in cotton. BMC plant biology 12: 1-19.

Zavattieri MA, Frederico AM, Lima M, Sabino R, Arnholdt-Schmitt B. 2010. Induction of somatic embryogenesis as an example of stress-related plant reactions. Electronic Journal of Biotechnology 13: 12-13.

Zuo W, Gu C, Zhang W, Xu K, Wang Y, Bai Y, Shan Y, Dai Q. 2019. Sewage sludge amendment improved soil properties and sweet sorghum yield and quality in a newly reclaimed mudflat land. Science of the Total Environment 654: 541-549. 


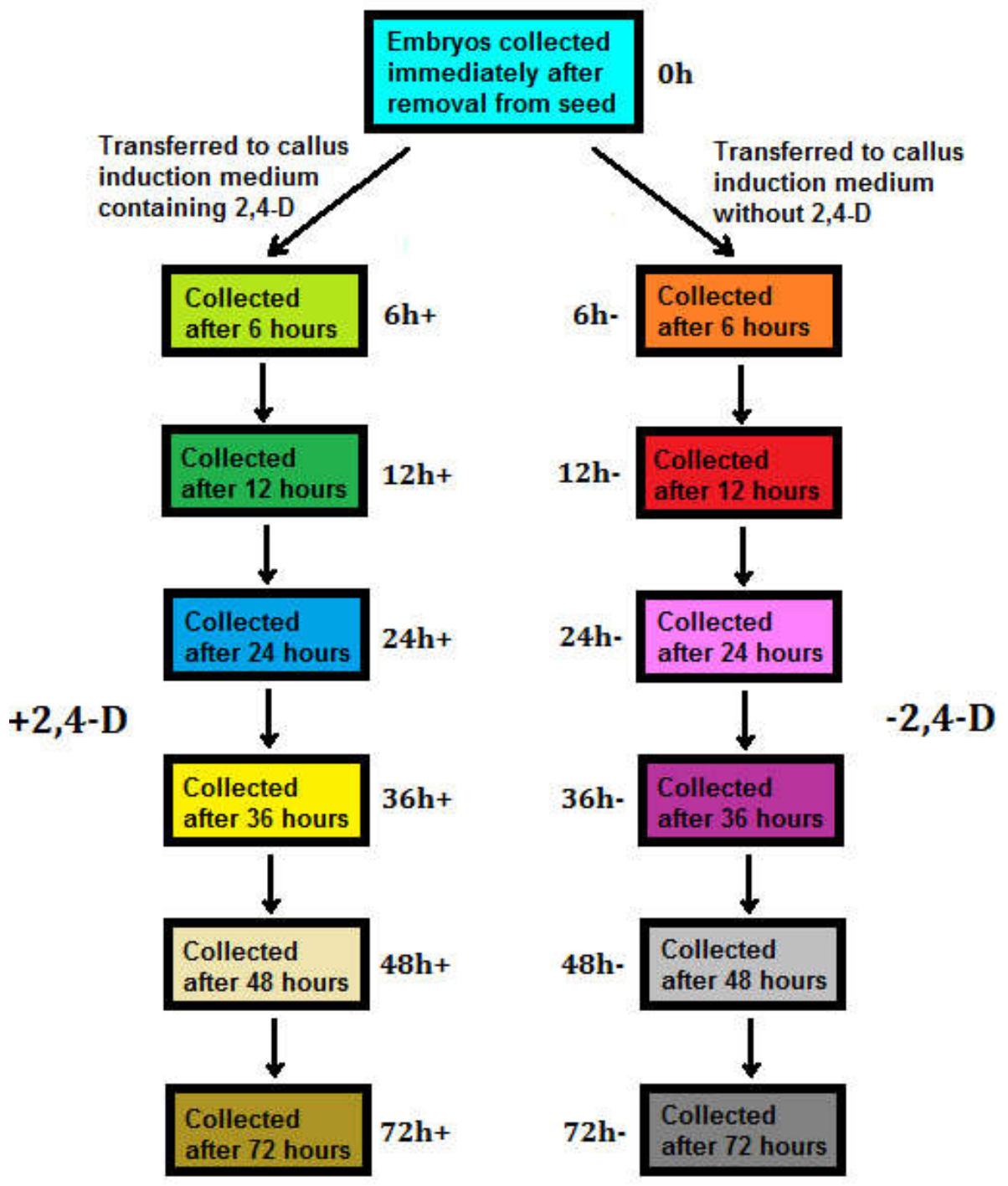

3 biological replicates per time point

Figure 2.1: Schematic of design for RNA-seq experiment 
A)

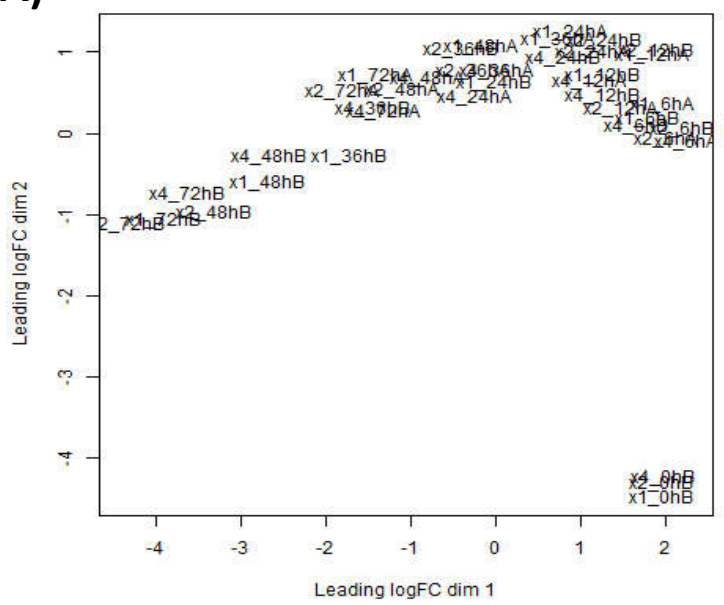

B)

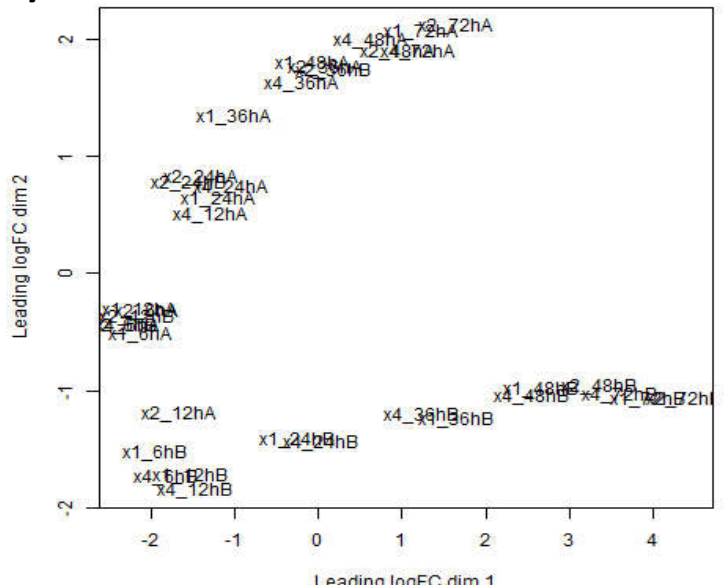

C)

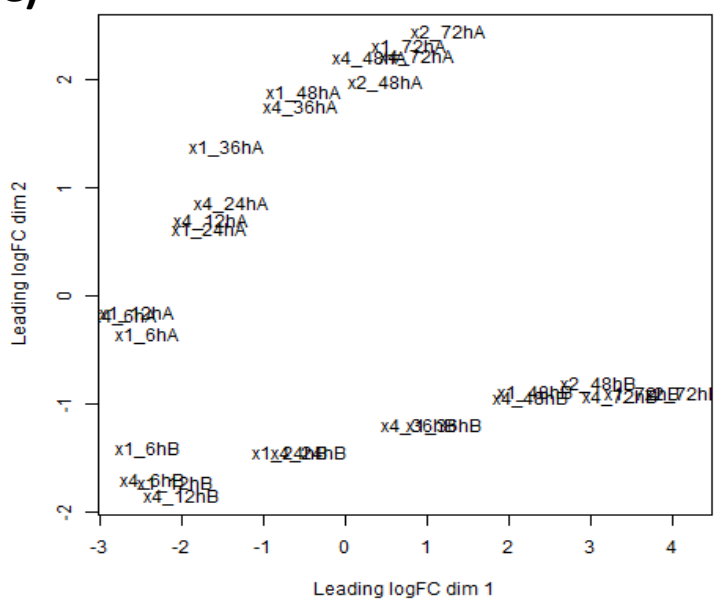

Figure 2.2: MDS plots showing relationships between samples. A) All samples included.

The 0-hour samples are far removed from all others. B) 0-hour samples eliminated. The second replicate samples (labels beginning with " 2 ") for the 6, 12, 24, and 36-hour time- 
points in the control condition (labels ending with " $\mathrm{B}$ ") cluster with the treatment condition (labels ending with "A"). C) MDS plot with all second replicate samples at the 6, 12, 24, and 36-hour time-points eliminated in both conditions. 


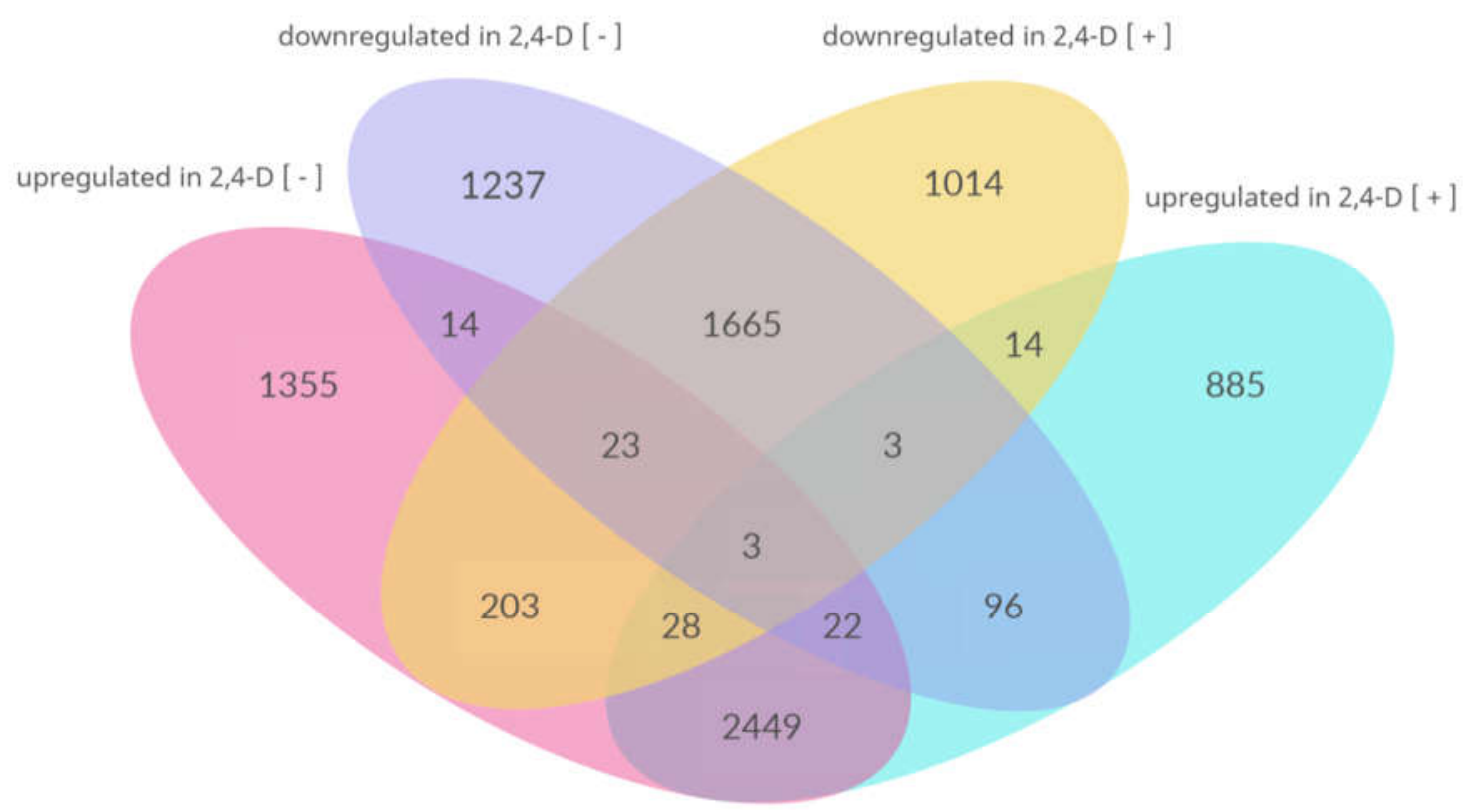

Figure 2.3: Sets of genes differentially expressed over time within the same experimental condition 


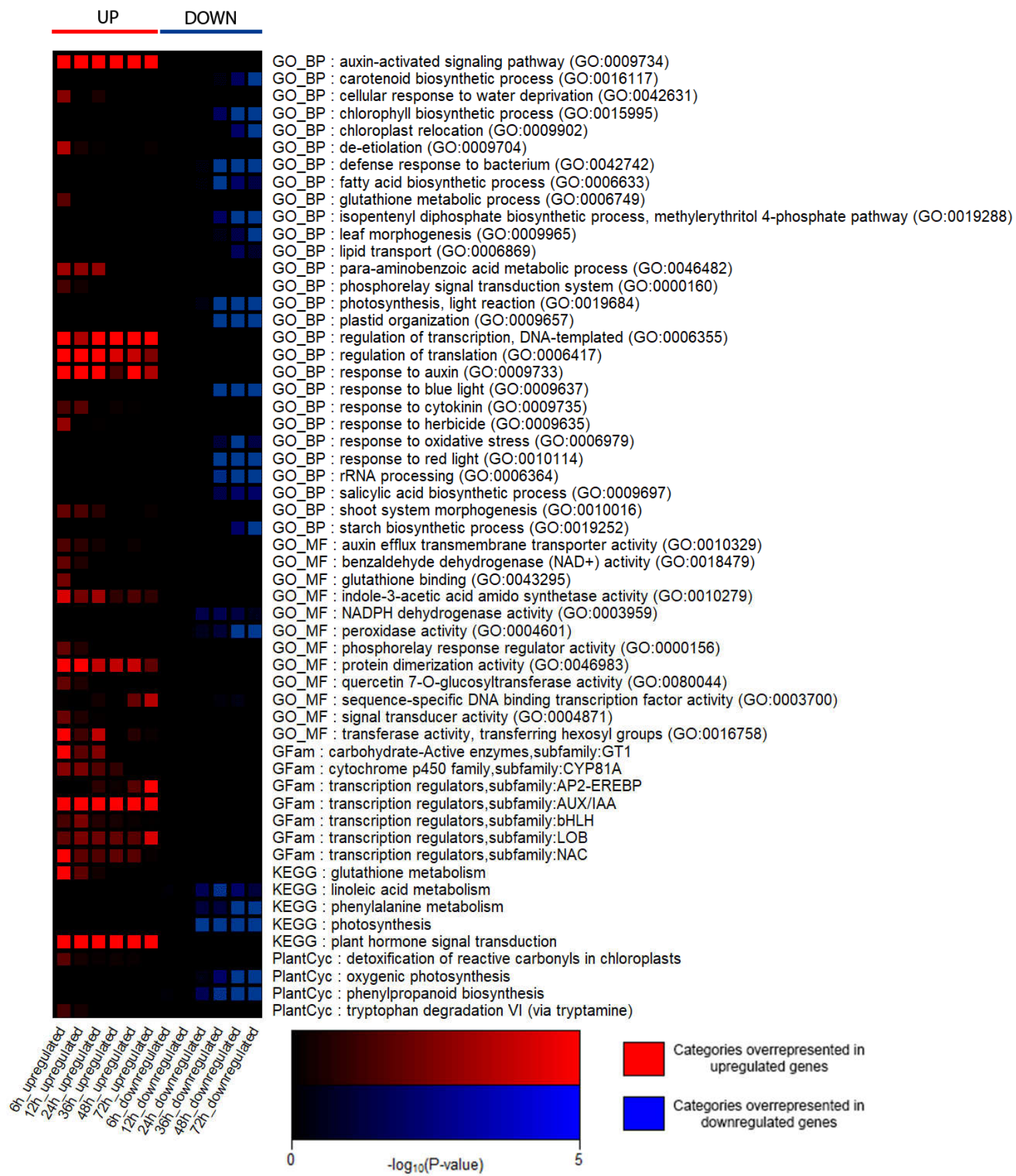

Figure 2.4: Selected overrepresented gene sets among upregulated and downregu-

lated genes at each cross-condition contrast. Degree of enrichment for each category is represented by taking the $-\log _{10}$ transformation of the $P$-value derived from a Fisher's exact test of the up- or down-regulated genes in each contrast versus the background of the sorghum genome. 

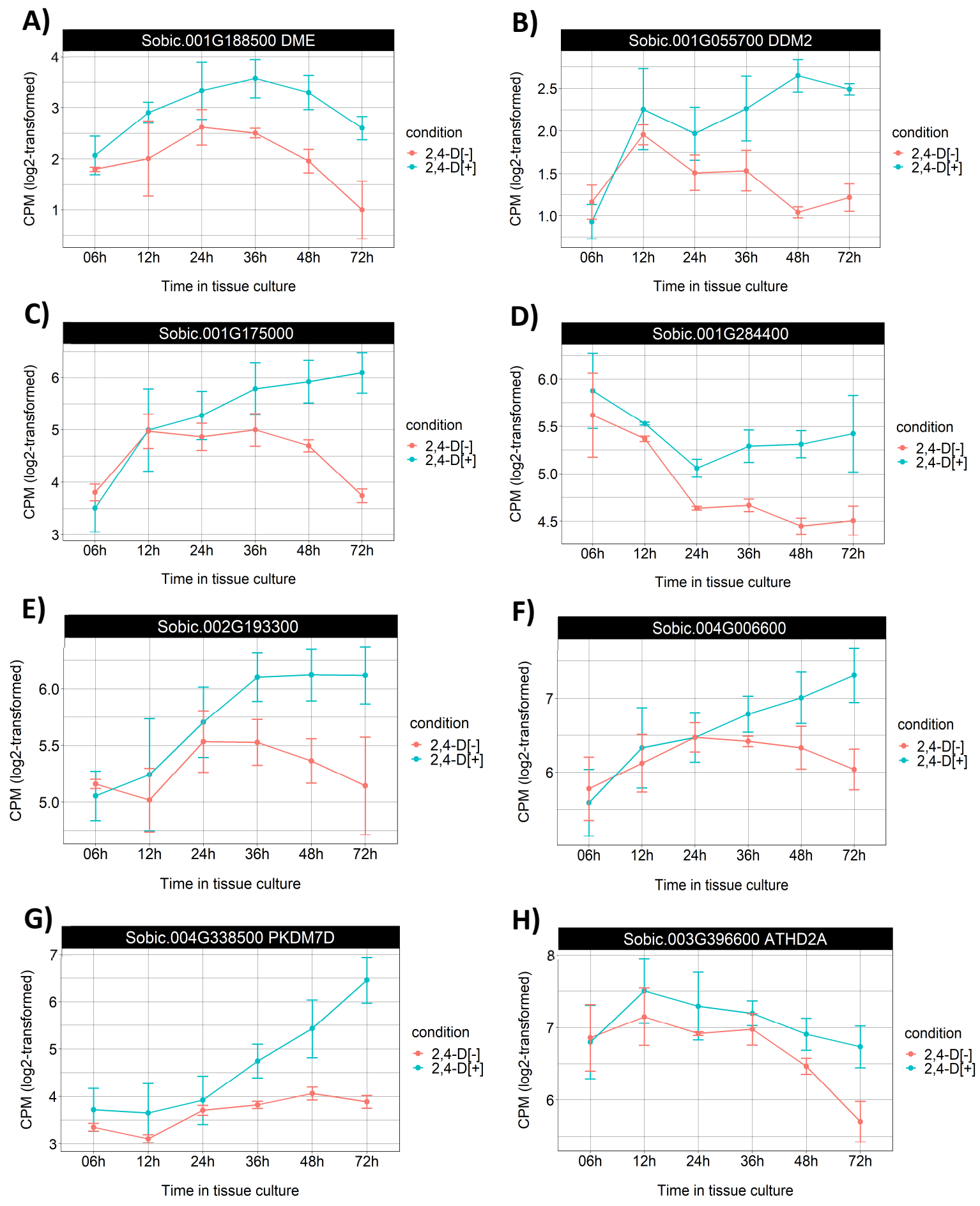

Figure 2.5: Expression of all epigenetic modulator genes upregulated across condi-

tions. A) An ortholog of DEMETER, a gene involved in DNA demethylation. B) An 
ortholog of DDM2 (also known as METHYLTRANSFERASE 1 that methylates DNA at CpG islands. C-G) JmjC domain-containing orthologs of H3K4me3 and H3K9 histone demethylases genes. H) An ortholog of a histone deacetylase gene. 

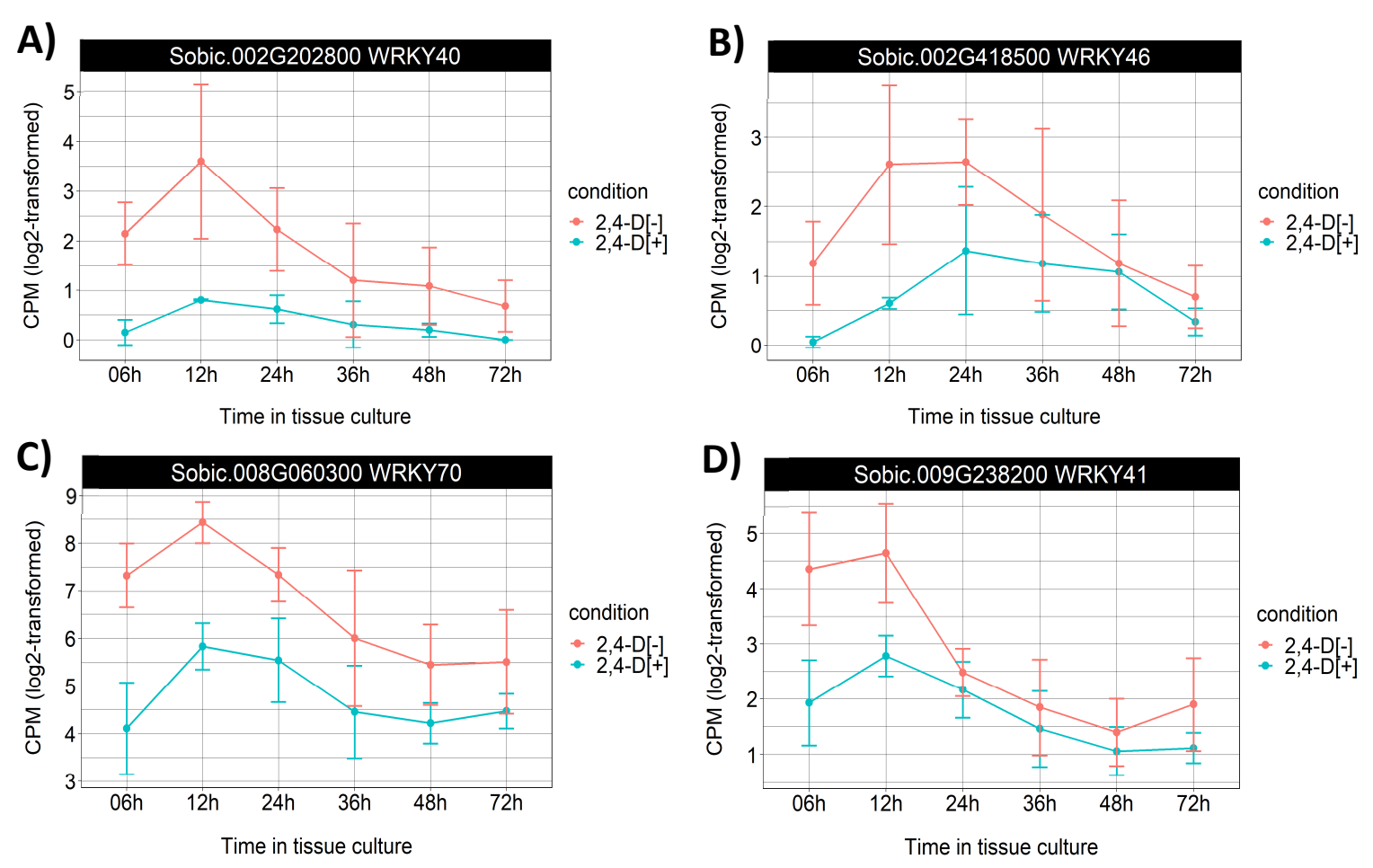

Figure 2.6: Expression of selected WRKY family transcription factor genes downregu-

lated across conditions. Several genes orthologous to WRKY transcription factor genes in Arabidopsis were downregulated in the treatment relative to the control condition in the first two contrasts made through time. 

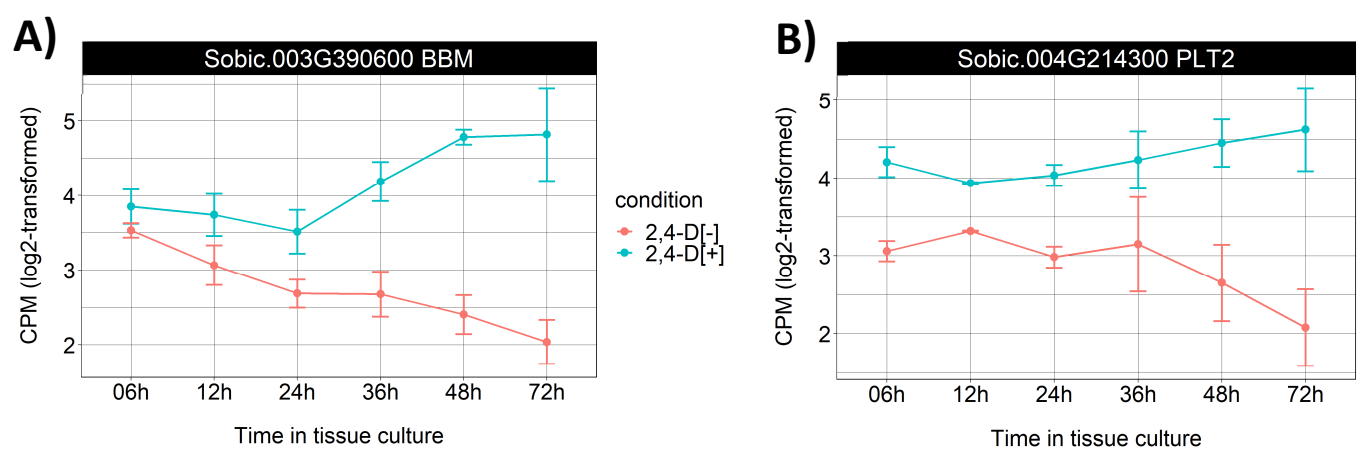

condition

$\rightarrow 2,4-D[-]$
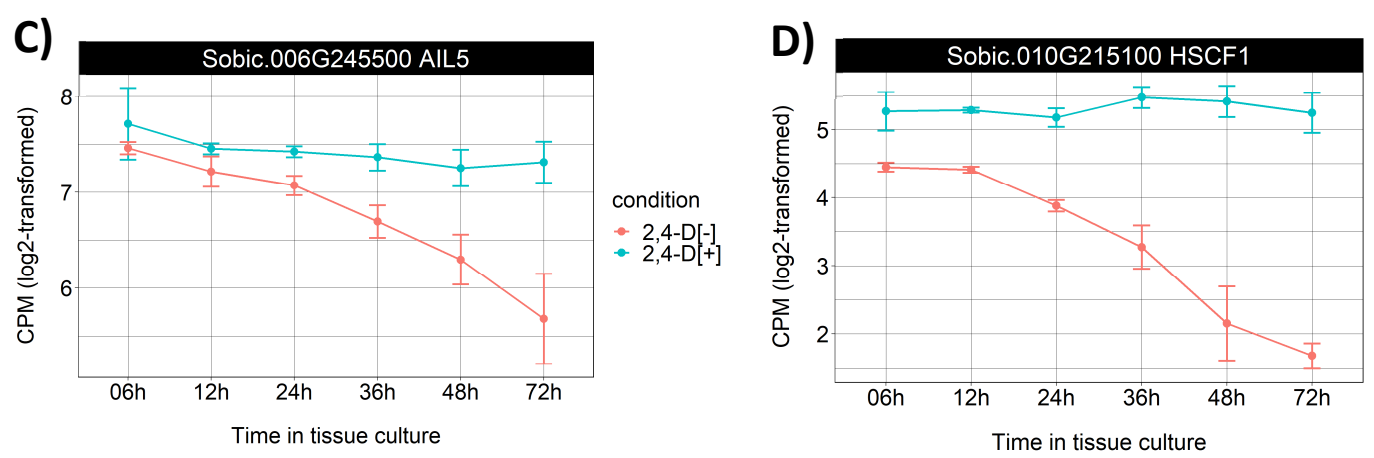

condition 2,4-D[-]
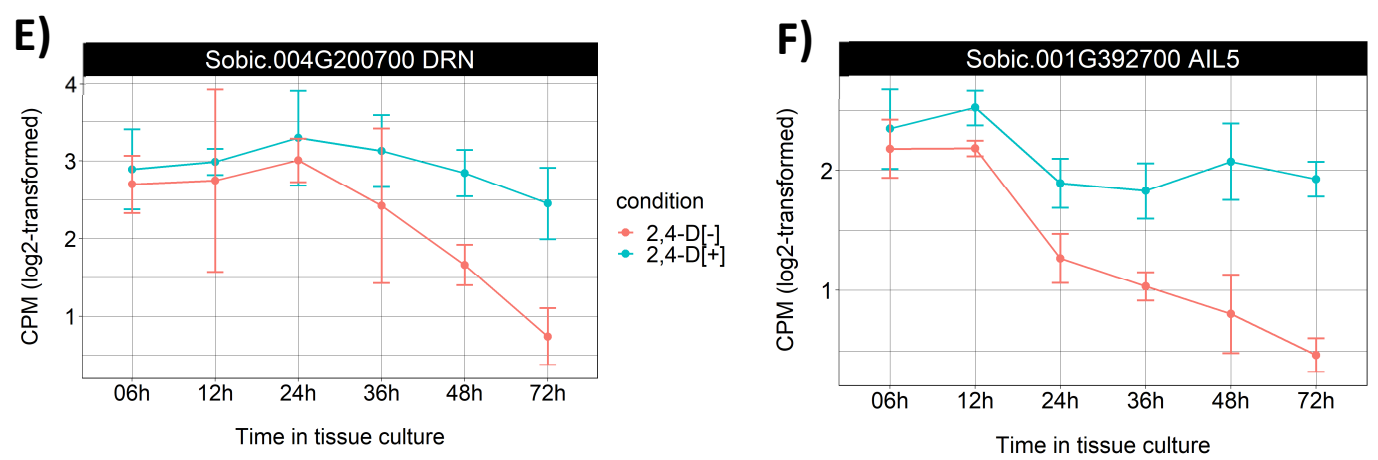

condition - 2,4-D[-]
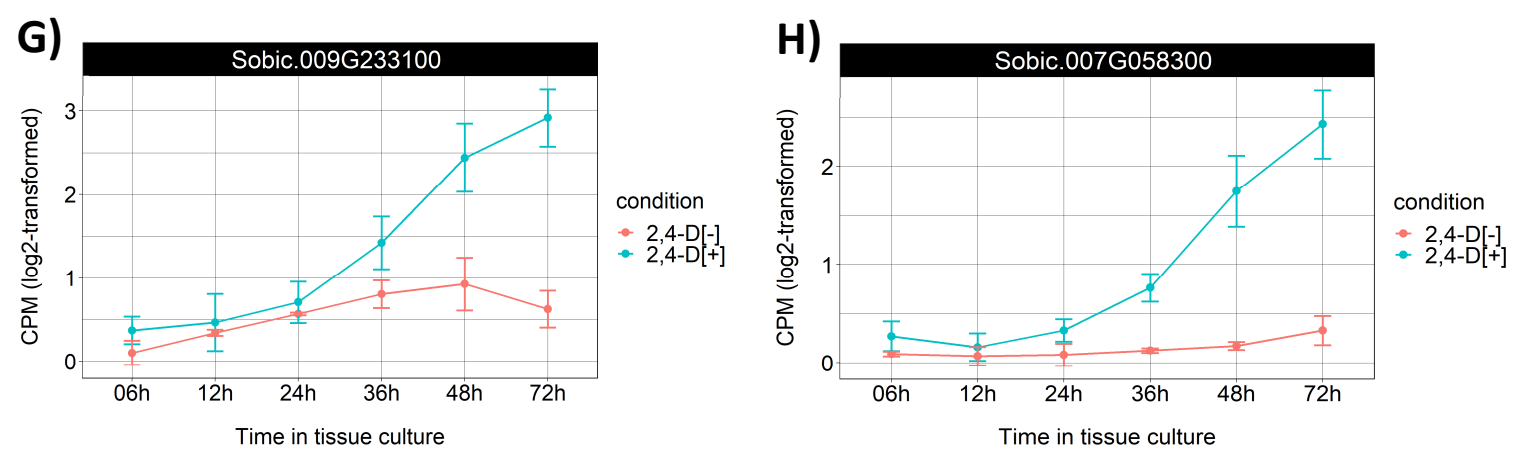

Figure 2.7: Expression of selected AP2/ERF family transcription factor genes upregu-

lated across conditions. Orthologs of A) $B A B Y B O O M$ and its close relatives B) PLETHORA 2, C and F) AINTEGUMENTA-like 5, D) Heat shock complementing factor 1, E) 
DORNROSCHEN, also known as ENHANCER OF SHOOT REGENERATION 1 , and $\mathbf{G}$ and $\mathbf{H}$ ) two unnamed AP2-like transcription factor genes. 

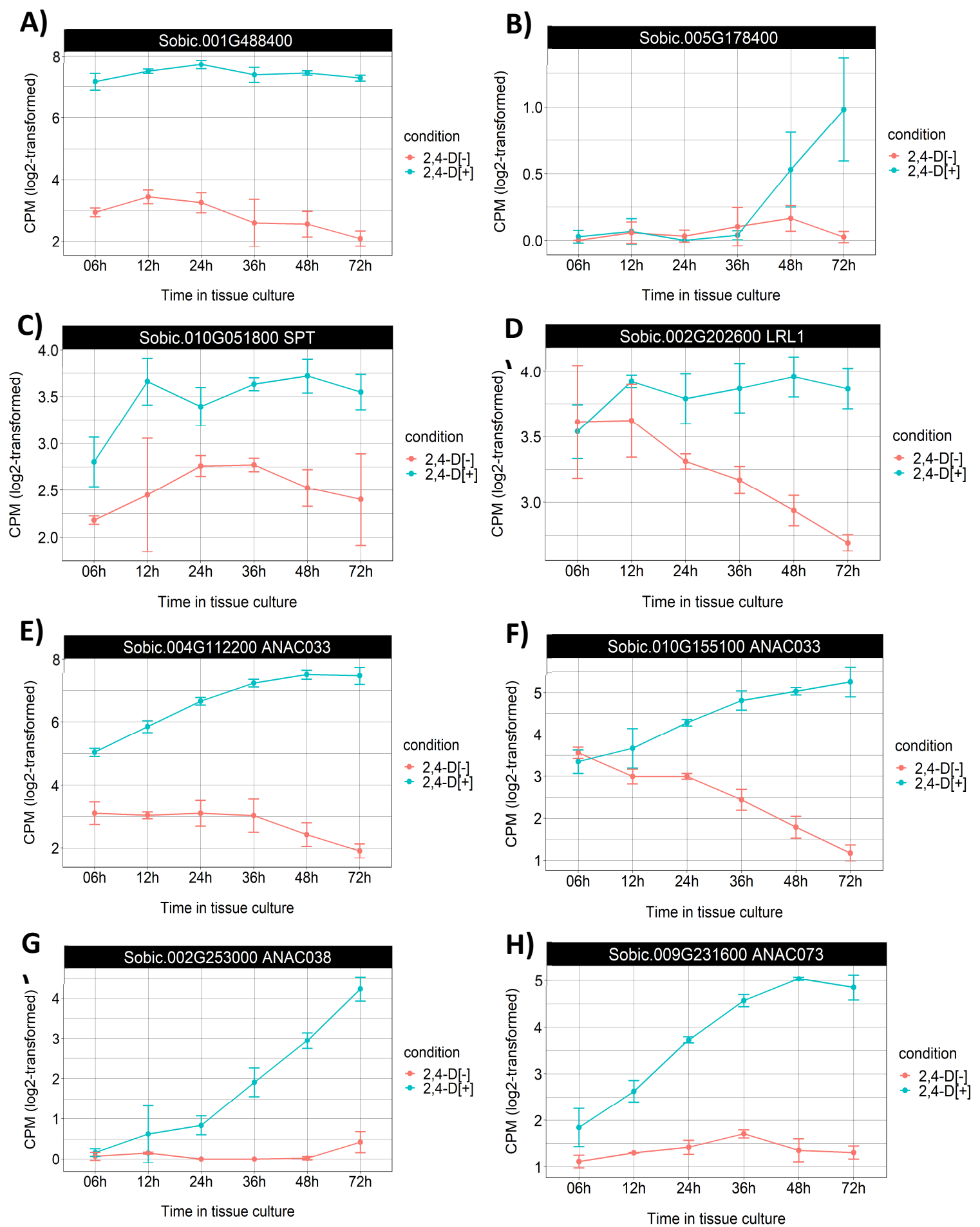

Figure 2.8: Expression of selected bHLH and NAC domain-containing transcription fac-

tor genes upregulated across conditions. A-D) Orthologs of unnamed members of the 
bHLH transcription factor family. E-H) Orthologs of members of the NAC domain family of transcription factors. E) and F) are orthologs of the SOMBRERO (SMB) gene known to be involved in root cap development in Arabidopsis. 

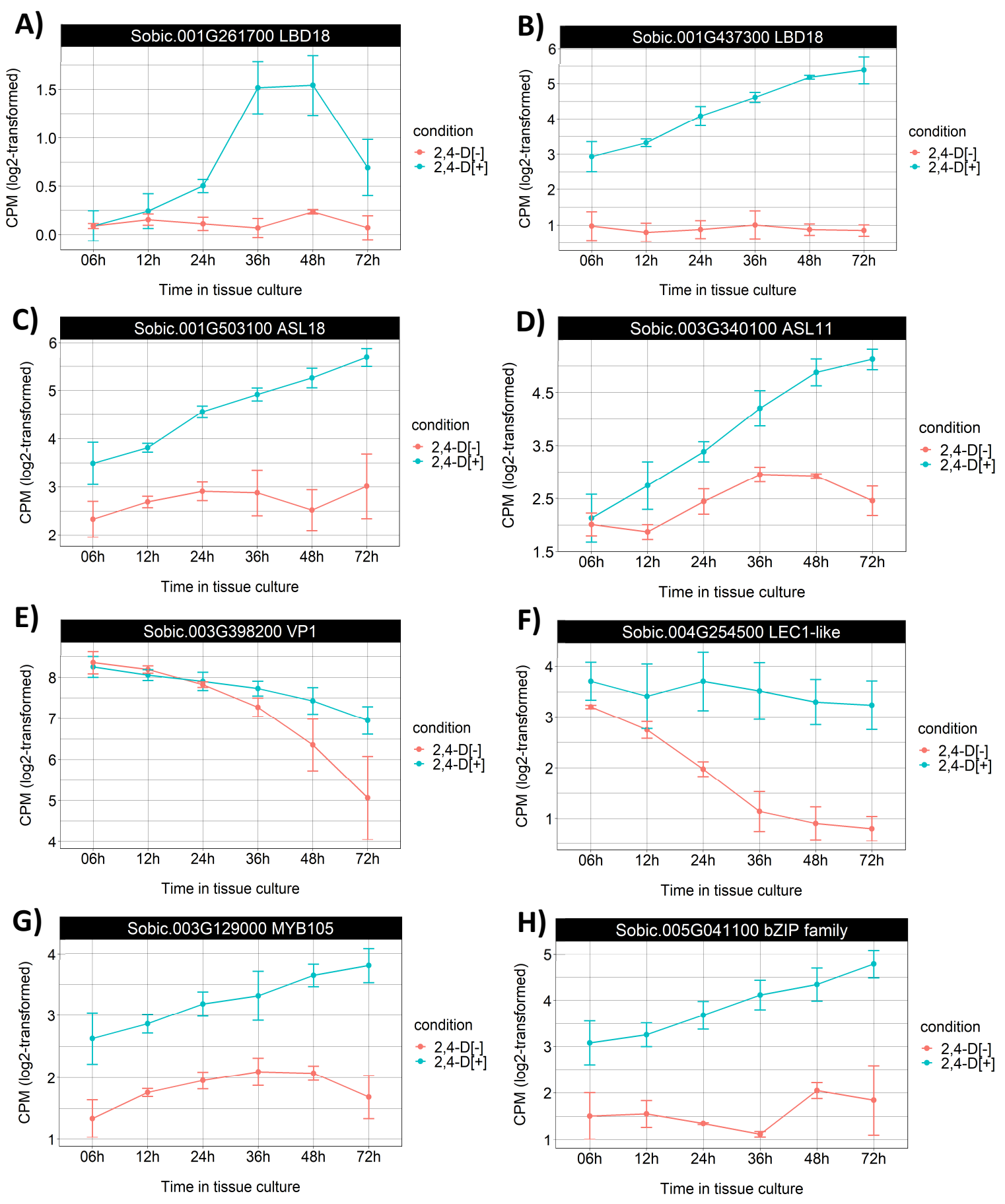

Figure 2.9: Expression of selected transcription factor genes from various families up-

regulated across conditions. A-D) LOB domain transcription factor genes including orthologs of, A) and B), LATERAL ORGAN BOUNDARIES DOMAIN 18 (LBD18), C) ASYM-

METRIC LEAVES2-LIKE 18, also known as LBD16, and D) ASL11, also known as LBD15. E) 
An ortholog of the B3 domain gene VIVIPAROUS 1. F) An ortholog of the nuclear factor Y protein LEAFY COTYLEDON 1-like. G) An ortholog of the MYB-related transcription factor gene MYB105. H) An ortholog of an unnamed member of the bZIP transcription factor family. 

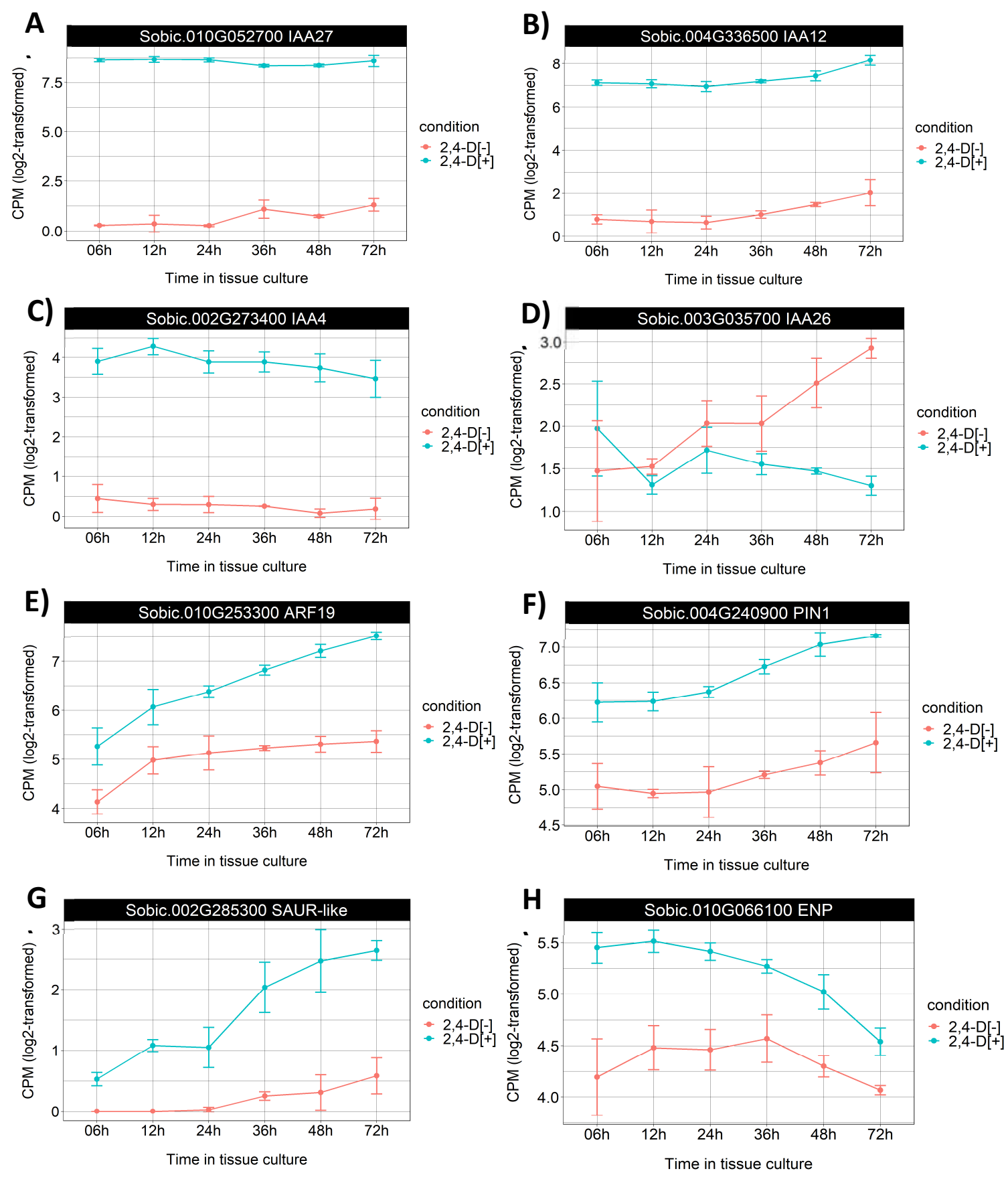

Figure 2.10: Expression curves of selected differentially expressed auxin-related genes.

A-D) Orthologs of $A U X / I A A$ genes. A), B), and C) show early response IAA genes that were strongly upregulated across conditions throughout the time-course. D) An ortholog of IAA26 was among the 3 IAA genes that were downregulated beginning at 
the 48-hour time-point. E) AUXIN RESPONSE FACTOR 19 (ARF19), a gene known to directly regulate $L B D 16$ and 29 , was upregulated at all time-points. Orthologs of the auxin efflux carrier gene PIN-FORMED 1 (PIN1) such as in F), were also strongly upregulated from an early time. Several orthologs of unnamed members of the Small Auxin Upregulated RNA (SAUR) gene family as in G) were also strongly induced. H) An ortholog of the ENHANCER OF PINOID (ENP) gene, a BTB/POZ domain protein involved in regulating the directional transport of auxin. 

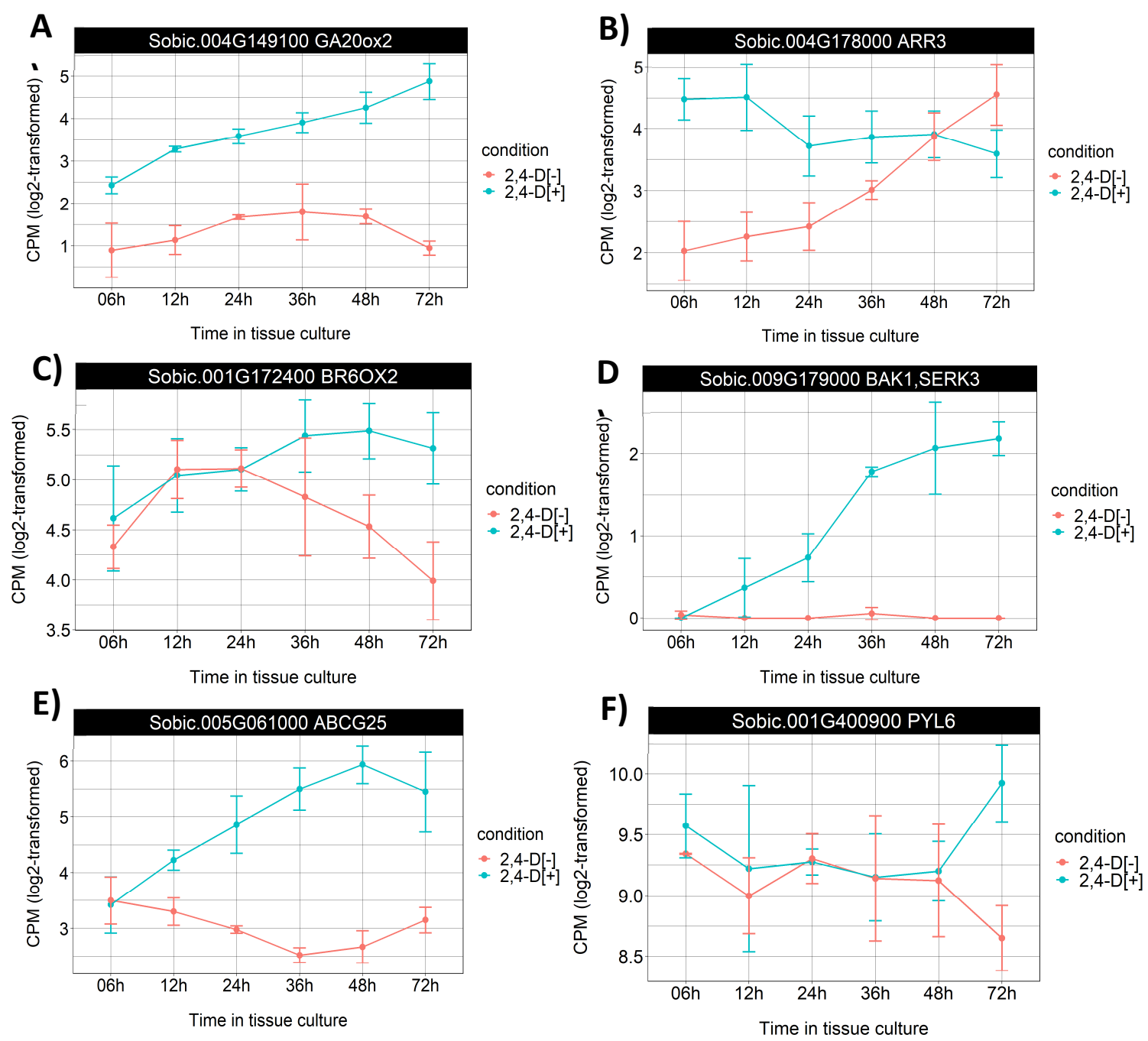

Figure 2.11: Expression curves of selected differentially expressed hormone (other

than auxin)-related genes. Orthologs of several genes involved in gibberellin metabo-

lism including the GA20 oxidase 2 gene shown in A) were upregulated across conditions.

5 orthologs of the ARABIDOPSIS RESPONSE REGULATOR (ARR) genes involved in cyto-

kinin signaling, such as $A R R 3$ as seen in B) were upregulated across conditions in the earlier time-points. C) An ortholog of a gene involved in brassinosteroid biosynthesis (BR60X2) was significantly upregulated beginning at the 48-hour time-point. D) a gene orthologous to BRI1-associated receptor kinase (BAK1) in rice (also known as SOMATIC 
EMBRYOGENESIS RECEPTOR-LIKE KINASE 3), which is involved in the brassinosteroid signaling pathway, was upregulated at all time-points following 6 hours from a baseline of near-zero read counts in the control condition. E) An ortholog of ATP-BINDING CASETTE FAMILY G 25 (ABCG25), an exporter of abscisic acid was upregulated beginning from the 24-hour time-point. Multiple orthologs of PYR1-like 6 (PYL6), a regulatory component of the abscisic acid receptor, such as the one displayed in F), were upregulated at the 72hour time-point. 

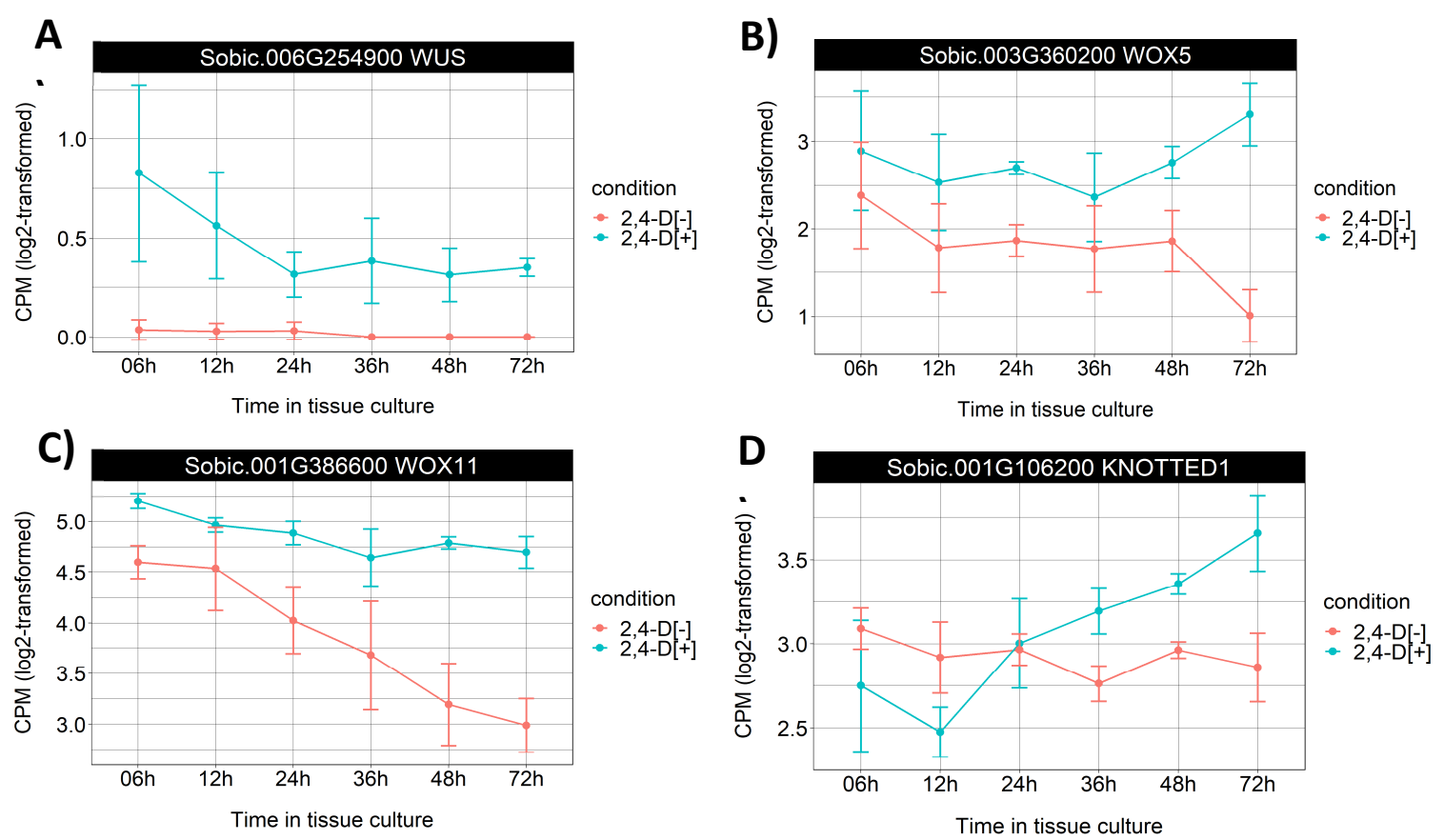

Figure 2.12: Expression curves of selected homeodomain-like superfamily genes. A)

The sorghum ortholog of WUSCHEL was expressed at extremely low levels in explant tissues. Though the per-gene read counts through time were apparently higher in the treatment condition, the gene was not differentially expressed to a statistically significant degree. Orthologs of B) WUSCHEL related homeobox 5 (WOX5), differentially expressed beginning at the 48-hour time-point, and C) WOX11, differentially expressed beginning at the 24-hour time-point. D) An ortholog of the maize KNOTTED1, a homeobox protein involved in regulation of the shoot apical meristem, which showed a maximum $\log _{2}$ fold-change just below the cutoff of $1(0.95)$ at the 72 -hour time-point. 
Table 2.1: Summary of RNA-seq data analysis

\begin{tabular}{|c|c|c|c|c|c|c|}
\hline sample & $\begin{array}{l}\text { total raw } \\
\text { reads }\end{array}$ & $\begin{array}{l}\text { total reads } \\
\text { remaining } \\
\text { after trim- } \\
\text { ming }\end{array}$ & $\begin{array}{l}\text { total reads } \\
\text { remaining } \\
\text { after clean- } \\
\text { ing }\end{array}$ & $\begin{array}{l}\text { reads } \\
\text { aligning } \\
\text { uniquely }\end{array}$ & \begin{tabular}{|l|} 
unique \\
alignments \\
assigned to \\
a gene
\end{tabular} & $\begin{array}{l}\text { percentage } \\
\text { of raw } \\
\text { reads used } \\
\text { in DE anal- } \\
\text { ysis }\end{array}$ \\
\hline 1-Oh- & $30,678,101$ & $30,307,011$ & $12,351,667$ & $11,044,651$ & $10,345,312$ & $33.72 \%$ \\
\hline 2-Oh- & $24,304,459$ & $23,355,897$ & $11,997,970$ & $10,951,083$ & $10,337,197$ & $42.53 \%$ \\
\hline 4-Oh- & $33,057,223$ & $32,610,572$ & $12,368,255$ & $11,056,229$ & $10,115,392$ & $30.60 \%$ \\
\hline 1-6h+ & $25,404,215$ & $25,101,907$ & $22,922,917$ & $20,612,613$ & $19,533,010$ & $76.89 \%$ \\
\hline $2-6 h+$ & $23,080,333$ & $22,742,131$ & $19,944,184$ & $18,178,647$ & $17,104,228$ & $74.11 \%$ \\
\hline $4-6 h+$ & $22,892,530$ & $22,533,547$ & $22,210,796$ & $20,336,537$ & $19,322,161$ & $84.40 \%$ \\
\hline 1-6h- & $25,701,380$ & $25,394,451$ & $15,738,273$ & $13,704,501$ & \begin{tabular}{|l|}
$12,926,052$ \\
\end{tabular} & $50.29 \%$ \\
\hline $2-6 h-$ & $24,117,353$ & $23,782,501$ & $21,485,653$ & $19,565,884$ & $18,534,473$ & $76.85 \%$ \\
\hline 4-6h- & $23,255,626$ & $22,924,082$ & $22,447,972$ & $20,567,400$ & $19,515,199$ & $83.92 \%$ \\
\hline $1-12 \mathrm{~h}+$ & $28,831,048$ & $28,438,758$ & $27,550,707$ & $24,960,525$ & $23,716,245$ & $82.26 \%$ \\
\hline $2-12 h+$ & $26,627,184$ & $25,992,074$ & $15,103,361$ & $13,513,872$ & $12,844,333$ & $48.24 \%$ \\
\hline $4-12 h+$ & $24,198,130$ & $23,812,912$ & $23,614,685$ & $21,585,033$ & $20,493,402$ & $84.69 \%$ \\
\hline 1-12h- & $27,684,813$ & $27,357,453$ & $24,165,802$ & $21,782,282$ & 978 & $74.37 \%$ \\
\hline $2-12 \mathrm{~h}-$ & $27,015,888$ & $26,658,710$ & $25,100,399$ & $22,856,949$ & $21,672,544$ & $80.22 \%$ \\
\hline $4-12 \mathrm{~h}-$ & $28,608,590$ & $28,202,020$ & $28,008,533$ & $25,663,149$ & $24,418,301$ & $85.35 \%$ \\
\hline $1-24 \mathrm{~h}+$ & $26,813,313$ & $26,455,148$ & $24,834,901$ & $22,579,097$ & $21,422,177$ & $79.89 \%$ \\
\hline $2-24 h+$ & $26,401,893$ & $26,092,705$ & $25,418,860$ & 9,068 & 628 & \\
\hline $4-24 h+$ & $21,359,652$ & $21,060,210$ & $20,880,198$ & $19,081,590$ & $18,199,244$ & $85.20 \%$ \\
\hline $1-24 h-$ & $29,925,128$ & $29,560,103$ & $29,235,602$ & $26,858,487$ & $25,502,974$ & $85.22 \%$ \\
\hline $2-24 h-$ & $28,076,711$ & $27,733,995$ & $26,394,874$ & $24,091,837$ & $22,877,513$ & $81.48 \%$ \\
\hline 4-24h- & $26,232,347$ & $25,858,164$ & $25,627,310$ & $23,517,614$ & $22,304,006$ & $85.02 \%$ \\
\hline $1-36 h+$ & $33,626,422$ & $33,223,339$ & $32,836,060$ & $30,273,971$ & $28,762,522$ & $85.54 \%$ \\
\hline $2-36 h+$ & $25,494,654$ & $25,147,071$ & $24,812,130$ & $22,601,856$ & $21,432,524$ & $84.07 \%$ \\
\hline 4-36h+ & $22,130,637$ & $21,819,241$ & $21,598,232$ & $19,762,045$ & $18,769,586$ & $84.81 \%$ \\
\hline 1-36h- & $30,035,246$ & $29,684,110$ & $29,415,797$ & $27,077,345$ & $25,733,644$ & $85.68 \%$ \\
\hline 2-36h- & $26,379,244$ & $26,024,746$ & $25,062,237$ & $22,850,911$ & $21,738,161$ & $82.41 \%$ \\
\hline 4-36h- & $23,157,022$ & $22,811,395$ & $22,637,749$ & $20,726,863$ & $19,704,660$ & $85.09 \%$ \\
\hline $1-48 \mathrm{~h}+$ & $27,021,076$ & $26,697,463$ & $26,367,907$ & $24,256,185$ & $22,982,272$ & $85.05 \%$ \\
\hline $2-48 h+$ & $27,282,588$ & $26,900,704$ & $24,182,174$ & $21,954,223$ & $20,736,586$ & $76.01 \%$ \\
\hline $4-48 h+$ & $25,950,084$ & $25,580,112$ & $25,159,728$ & $23,042,176$ & $21,942,730$ & $84.56 \%$ \\
\hline 1-48h- & $29,514,191$ & $29,087,466$ & $28,670,868$ & $26,434,511$ & $25,068,020$ & $84.94 \%$ \\
\hline $2-48 \mathrm{~h}-$ & $24,385,914$ & $24,050,897$ & $23,653,323$ & $21,525,629$ & $20,385,161$ & $83.59 \%$ \\
\hline 4-48h- & $20,452,906$ & $19,368,764$ & $19,187,802$ & $17,532,132$ & $16,670,043$ & $81.50 \%$ \\
\hline $1-72 \mathrm{~h}+$ & $29,714,647$ & $29,320,138$ & $28,051,945$ & $25,703,840$ & $24,311,120$ & $81.82 \%$ \\
\hline $2-72 \mathrm{~h}+$ & $22,816,932$ & $22,459,456$ & $21,755,940$ & $19,662,641$ & $18,648,180$ & $81.73 \%$ \\
\hline $4-72 \mathrm{~h}+$ & $19,099,251$ & $18,102,652$ & $17,853,520$ & $16,174,035$ & $15,341,869$ & $80.33 \%$ \\
\hline 1-72h- & $21,607,330$ & $20,308,564$ & $19,715,370$ & $18,076,725$ & $17,099,413$ & $79.14 \%$ \\
\hline
\end{tabular}




\begin{tabular}{|l|l|l|l|l|l|l|}
\hline $2-72 \mathrm{~h}-$ & $23,571,537$ & $23,233,399$ & $22,295,049$ & $20,339,993$ & $19,272,626$ & $81.76 \%$ \\
\hline $4-72 \mathrm{~h}-$ & $22,209,370$ & $21,875,931$ & $21,656,514$ & $19,773,013$ & $18,692,199$ & $84.16 \%$ \\
\hline
\end{tabular}


Table 2.2: Most highly upregulated genes in contrasts made across time.

\begin{tabular}{|c|c|c|c|c|c|}
\hline Gene ID & $\begin{array}{l}\text { peak } \\
\log _{2} \text { (fold } \\
\text { change) }\end{array}$ & $\begin{array}{l}\text { contrast } \\
\text { with peak } \\
\text { log2(fold } \\
\text { change) }\end{array}$ & chromosomal coordinates & $\begin{array}{l}\text { closest match in Ara- } \\
\text { bidopsis }\end{array}$ & Description \\
\hline Sobic.005G112400 & 14.36 & 72h-vs.6h- & Chr05:38567082-38568601(+) & AT1G29090 & Cysteine proteinases superfamily protein \\
\hline Sobic.010G234500 & 13.53 & 72h-vs.6h- & Chr10:57725721-57727469(-) & AT4G35160 & O-methyltransferase family protein \\
\hline Sobic.006G150100 & 13.48 & 72h-vs.6h- & Chr06:51145123-51147729(+) & PIP2;2 & plasma membrane intrinsic protein 2 \\
\hline Sobic.005G050200 & 13.27 & 72h-vs.6h- & Chr05:4770080-4771218(-) & [no close hit] & \\
\hline Sobic.005G049600 & 13.25 & 72h-vs.6h- & Chr05:4708347-4713388(-) & WAK5 & wall associated kinase 5 \\
\hline Sobic.006G277500 & 13.24 & 72h-vs.6h- & Chr06:60834877-60837191(-) & AT1G71695 & Peroxidase superfamily protein \\
\hline Sobic.003G059000 & 13.06 & 72h-vs.6h- & Chr03:5185672-5186593(+) & AT5G05500 & Pollen Ole e 1 allergen and extensin family protein \\
\hline Sobic.002G421300 & 13.01 & 72h-vs.6h- & Chr02:76860176-76861505(+) & AT2G39050 & hydroxyproline-rich glycoprotein family protein \\
\hline Sobic.001G231800 & 12.77 & 48h-vs.6h- & Chr01:22398330-22399091(+) & [no close hit] & \\
\hline Sobic.004G292900 & 12.74 & 72h-vs.6h- & Chr04:63330856-63331736(-) & AT2G45180 & $\begin{array}{l}\text { Bifunctional inhibitor/lipid-transfer protein/seed storage } \\
2 \mathrm{~S} \text { albumin superfamily protein }\end{array}$ \\
\hline Sobic.new. 230 & 12.70 & 24h-vs.6h- & Chr01:2368735-2370876(+) & [not protein coding] & \\
\hline Sobic.002G391300 & 12.56 & 72h-vs.6h- & Chr02:74404889-74406451(-) & AT4G11290 & Peroxidase superfamily protein \\
\hline Sobic.002G065600 & 12.54 & $72 \mathrm{~h}+\mathrm{vs} .6 \mathrm{~h}+$ & Chr02:6476747-6477840(-) & TPS21 & terpene synthase 21 \\
\hline Sobic.001G224300 & 12.17 & $48 \mathrm{~h}+\mathrm{vs} .6 \mathrm{~h}+$ & Chr01:21485582-21489706(-) & CESA4 & cellulose synthase A4 \\
\hline Sobic.007G058800 & 12.07 & $72 \mathrm{~h}+\mathrm{vs} .6 \mathrm{~h}+$ & Chr07:6103097-6107178(+) & OMT1 & O-methyltransferase 1 \\
\hline Sobic.007G090460 & 11.54 & $72 \mathrm{~h}+\mathrm{vs} .6 \mathrm{~h}+$ & Chr07:13940089-13941408(+) & XTH13 & xyloglucan endotransglucosylase/hydrolase 13 \\
\hline Sobic.006G172500 & 11.46 & 48h-vs.6h- & Chr06:52842536-52843272(+) & AT4G12520 & $\begin{array}{l}\text { Bifunctional inhibitor/lipid-transfer protein/seed storage } \\
2 \mathrm{~S} \text { albumin superfamily protein }\end{array}$ \\
\hline Sobic.001G304201 & 11.39 & 48h-vs.6h- & Chr01:58813600-58814288(+) & AT5G46900 & $\begin{array}{l}\text { Bifunctional inhibitor/lipid-transfer protein/seed storage } \\
2 \mathrm{~S} \text { albumin superfamily protein }\end{array}$ \\
\hline Sobic.003G096100 & 11.16 & $72 \mathrm{~h}+\mathrm{vs} .6 \mathrm{~h}+$ & Chr03:8499492-8501691(-) & WAT1 & Walls Are Thin 1 \\
\hline Sobic.K026900 & 11.07 & $72 h+v s .6 h+$ & super_2674:1128-2880(+) & AT3G21770 & Peroxidase superfamily protein \\
\hline Sobic.005G110436 & 11.02 & 48h-vs.6h- & Chr05:26524730-26529301(+) & AT1G78060 & Glycosyl hydrolase family protein \\
\hline Sobic.005G073000 & 10.96 & 48h-vs.6h- & Chr05:8788684-8789862(-) & [no close hit] & \\
\hline
\end{tabular}




\begin{tabular}{|c|c|c|c|c|c|}
\hline Sobic.006G185000 & 10.76 & 48h-vs.6h- & Chr06:54004227-54009044(+) & [no close hit] & \\
\hline Sobic.007G073100 & 10.59 & 48h-vs.6h- & Chr07:8223474-8228407(-) & PAO1 & polyamine oxidase 1 \\
\hline Sobic.new.17186 & 10.56 & $12 \mathrm{~h}-\mathrm{vs} .6 \mathrm{~h}-$ & Chr08:56571701-56572222(.) & [not protein coding] & \\
\hline Sobic.new.1275 & 10.50 & $24 h-v s .6 h-$ & Chr01:16750728-16750971(.) & [not protein coding] & \\
\hline Sobic.008G076500 & 10.34 & $12 \mathrm{~h}-\mathrm{vs} .6 \mathrm{~h}-$ & Chr08:11506709-11507250(+) & [no close hit] & \\
\hline Sobic.005G169300 & 10.22 & $72 \mathrm{~h}+\mathrm{vs} .6 \mathrm{~h}+$ & Chr05:64817956-64818743(+) & PR4 & pathogenesis-related 4 \\
\hline Sobic.010G020200 & 10.22 & $72 \mathrm{~h}+\mathrm{vs} .6 \mathrm{~h}+$ & Chr10:1636425-1637327(+) & PR1 & pathogenesis-related gene 1 \\
\hline Sobic.003G082700 & 10.06 & 36h-vs.6h- & Chr03:7088406-7091241(+) & RXF12 & $\begin{array}{l}\text { glycosyl hydrolase family } 10 \text { protein / carbohydrate-bind- } \\
\text { ing domain-containing protein }\end{array}$ \\
\hline Sobic.003G235500 & 10.01 & 36h-vs.6h- & Chr03:57383232-57383791(-) & [no close hit] & \\
\hline Sobic.003G352800 & 9.93 & $48 h+v s .6 h+$ & Chr03:67174664-67177905(-) & LAC17 & laccase 17 \\
\hline Sobic.004G304200 & 9.74 & $36 h-v s .6 h-$ & Chr04:64323027-64324886(-) & AT1G01800 & NAD(P)-binding Rossmann-fold superfamily protein \\
\hline Sobic.003G100900 & 9.71 & $48 \mathrm{~h}+\mathrm{vs} .6 \mathrm{~h}+$ & Chr03:8923838-8925084(+) & [no close hit] & \\
\hline Sobic.006G232500 & 9.38 & $48 \mathrm{~h}+\mathrm{vs} .6 \mathrm{~h}+$ & Chr06:57493105-57495738(+) & BFN1 & bifunctional nuclease $\mathrm{i}$ \\
\hline Sobic.004G085800 & 9.24 & $48 \mathrm{~h}+\mathrm{vs} .6 \mathrm{~h}+$ & Chr04:7121291-7123548(+) & MC9 & metacaspase 9 \\
\hline Sobic.003G321400 & 8.98 & 24h-vs.6h- & Chr03:64892236-64894047(+) & ENODL9 & early nodulin-like protein 9 \\
\hline Sobic.007G091900 & 8.70 & $36 \mathrm{~h}+\mathrm{vs} .6 \mathrm{~h}+$ & Chr07:15544884-15549156(+) & AT5G57670 & Protein kinase superfamily protein \\
\hline Sobic.008G146700 & 8.69 & $24 h+v s .6 h+$ & Chr08:57941395-57943066(+) & BGL2 & beta-1,3-glucanase 2 \\
\hline Sobic.009G221800 & 8.68 & 24h-vs.6h- & Chr09:56484791-56487061(-) & AT3G18260 & Reticulon family protein \\
\hline Sobic.009G038300 & 8.66 & $36 \mathrm{~h}+\mathrm{vs} .6 \mathrm{~h}+$ & Chr09:3598643-3600689(-) & [no close hit] & \\
\hline Sobic.009G179000 & 8.60 & $36 \mathrm{~h}+\mathrm{vs} .6 \mathrm{~h}+$ & Chr09:53361509-53366268(+) & AT5G21090 & Leucine-rich repeat (LRR) family protein \\
\hline Sobic.006G003000 & 8.60 & $36 \mathrm{~h}+\mathrm{vs} .6 \mathrm{~h}+$ & Chr06:493293-495354(+) & AT1G63310 & \\
\hline Sobic.001G218400 & 8.52 & $24 h+v s .6 h+$ & Chr01:20327471-20329552(+) & AT3G17380 & TRAF-like family protein \\
\hline Sobic.005G075100 & 8.44 & 24h-vs.6h- & Chr05:9176676-9182428(+) & AT3G14470 & NB-ARC domain-containing disease resistance protein \\
\hline Sobic.008G182300 & 8.36 & 24h-vs.6h- & Chr08:61597723-61598752(-) & OSM34 & osmotin 34 \\
\hline Sobic.004G017000 & 8.20 & $24 \mathrm{~h}+\mathrm{vs} .6 \mathrm{~h}+$ & Chr04:1347959-1350371(-) & AT2G34930 & disease resistance family protein / LRR family protein \\
\hline
\end{tabular}




\begin{tabular}{|l|l|l|l|l|l|}
\hline Sobic.001G265800 & 7.71 & $24 \mathrm{~h}+\mathrm{vs} .6 \mathrm{~h}+$ & Chr01:50256348-50257358(+) & [no close hit] & \\
\hline Sobic.002G333900 & 7.68 & $12 \mathrm{~h}$-vs.6h- & Chr02:70227888-70231133(-) & CSLD3 & cellulose synthase-like D3 \\
\hline Sobic.005G127800 & 7.44 & $24 \mathrm{~h}+\mathrm{vs.6h+}$ & Chr05:55522152-55524303(-) & AT2G34930 & disease resistance family protein / LRR family protein \\
\hline Sobic.006G226800 & 7.41 & $24 \mathrm{~h}+\mathrm{vs.6h+}$ & Chr06:57175962-57178219(+) & BAN & NAD(P)-binding Rossmann-fold superfamily protein \\
\hline Sobic.003G199600 & 6.29 & $12 \mathrm{~h}+\mathrm{vs.6h+}$ & Chr03:52658842-52660006(+) & [no close hit] & \\
\hline Sobic.005G066100 & 6.25 & $12 \mathrm{~h}+\mathrm{vs.6h+}$ & Chr05:7460227-7461094(-) & [no close hit] & \\
\hline Sobic.005G217900 & 6.02 & $12 \mathrm{~h}-\mathrm{vs.6h-}$ & Chr05:70394964-70399350(+) & AT3G13510 & Protein of Unknown Function (DUF239) \\
\hline Sobic.003G031100 & 5.75 & $12 \mathrm{~h}+\mathrm{vs.6h+}$ & Chr03:2833693-2837326(-) & AT5G56960 & basic helix-loop-helix (bHLH) DNA-binding family protein \\
\hline Sobic.010G227300 & 5.75 & $12 \mathrm{~h}$-vs.6h- & Chr10:57032262-57036574(+) & NAC1 & NAC domain containing protein 1 \\
\hline Sobic.005G137200 & 5.65 & $12 \mathrm{~h}-\mathrm{vs.6h-}$ & Chr05:58985307-58986992(-) & CHS & Chalcone and stilbene synthase family protein \\
\hline
\end{tabular}


Table 2.3: Most highly downregulated genes in contrasts made across time.

\begin{tabular}{|c|c|c|c|c|c|}
\hline Gene ID & $\begin{array}{l}\text { peak } \\
\log _{2} \text { (fold } \\
\text { change) }\end{array}$ & $\begin{array}{l}\text { contrast } \\
\text { with peak } \\
\text { log2(fold } \\
\text { change) }\end{array}$ & chromosomal coordinates & $\begin{array}{l}\text { closest match in Ar- } \\
\text { abidopsis }\end{array}$ & Description \\
\hline Sobic.new.5133 & -13.52 & $12 \mathrm{~h}+\mathrm{vs} .6 \mathrm{~h}+$ & Chr02:62452730-62453844(.) & [no close hit] & \\
\hline Sobic.new.2302 & -12.13 & 24h-vs.6h- & Chr01:64040358-64040907(-) & [no close hit] & \\
\hline Sobic.new.20996 & -11.72 & 36h-vs.6h- & Chr10:225043-228433(+) & [no close hit] & \\
\hline Sobic.new.1563 & -11.21 & 24h-vs.6h- & Chr01:23217188-23226278(+) & AT2G05390 & putative retroelement pol polyprotein \\
\hline Sobic.new.17241 & -10.98 & $24 h+v s .6 h+$ & Chr08:57477889-57479686(-) & [no close hit] & \\
\hline Sobic.new.7548 & -10.41 & 24h-vs.6h- & Chr03:38413317-38413592(.) & [not protein coding] & \\
\hline Sobic.new.1651 & -10.40 & 36h-vs.6h- & Chr01:27119362-27119768(.) & [not protein coding] & \\
\hline Sobic.new.12535 & -10.30 & 36h-vs.6h- & Chr05:45486562-45509623(+) & [not protein coding] & \\
\hline Sobic.new.12453 & -9.57 & 36h-vs.6h- & Chr05:20029357-20037834(+) & [not protein coding] & \\
\hline Sobic.002G044500 & -9.54 & 36h-vs.6h- & Chr02:4211037-4212134(-) & ATCG00050 & ribosomal protein S16 \\
\hline Sobic.003G169300 & -9.50 & 24h-vs.6h- & Chr03:28855955-28856665(-) & ATCG00160 & ribosomal protein S2 \\
\hline Sobic.new.395 & -9.33 & 24h-vs.6h- & Chr01:4220565-4220785(.) & [not protein coding] & \\
\hline Sobic.new.15635 & -9.32 & 48h-vs.6h- & Chr07:49690777-49691040(.) & [not protein coding] & \\
\hline Sobic.new.311 & -8.89 & 24h-vs.6h- & Chr01:3291302-3291610(.) & [not protein coding] & \\
\hline Sobic.new.1717 & -8.77 & 24h-vs.6h- & Chr01:31355681-31355927(.) & [not protein coding] & \\
\hline Sobic.new.7546 & -8.69 & 24h-vs.6h- & Chr03:38376749-38377554(.) & [not protein coding] & \\
\hline Sobic.001G153700 & -8.65 & 72h-vs.6h- & Chr01:12354056-12355420(-) & ATL5 & RING/U-box superfamily protein \\
\hline Sobic.new.12619 & -8.63 & 36h-vs.6h- & Chr05:55836850-55837386(.) & [not protein coding] & \\
\hline Sobic.010G226700 & -8.61 & $72 h+v s .6 h+$ & Chr10:56933015-56933727(-) & [no close hit] & \\
\hline Sobic.new.16420 & -8.60 & 36h-vs.6h- & Chr08:831610-831974(.) & [not protein coding] & \\
\hline Sobic.010G136300 & -8.58 & $72 h+v s .6 h+$ & Chr10:20628782-20630945(+) & MFT & $\begin{array}{l}\text { PEBP (phosphatidylethanolamine-binding protein) family } \\
\text { protein }\end{array}$ \\
\hline Sobic.new.15522 & -8.52 & $24 h+v s .6 h+$ & Chr07:17952432-17952947(+) & [not protein coding] & \\
\hline Sobic.new.5124 & -8.51 & $48 h+v s .6 h+$ & Chr02:62309473-62310199(-) & PUP3 & purine permease 3 \\
\hline
\end{tabular}




\begin{tabular}{|c|c|c|c|c|c|}
\hline Sobic.new.13414 & -8.41 & 36h-vs.6h- & Chr06:39892155-39892458(.) & [not protein coding] & \\
\hline Sobic.new.1245 & -8.31 & $24 h+v s .6 h+$ & Chr01:15401556-15402674(.) & OMT1 & O-methyltransferase 1 \\
\hline Sobic.002G211700 & -8.30 & $36 \mathrm{~h}+\mathrm{vs} .6 \mathrm{~h}+$ & Chr02:60423442-60424313(+) & [no close hit] & \\
\hline Sobic.new.5187 & -8.13 & $48 \mathrm{~h}+\mathrm{vs} .6 \mathrm{~h}+$ & Chr02:63184452-63184839(-) & [not protein coding] & \\
\hline Sobic.010G259300 & -7.98 & 48h-vs.6h- & Chr10:59634426-59636119(+) & AT5G17540 & HXXXD-type acyl-transferase family protein \\
\hline Sobic.new.1971 & -7.82 & $36 h+v s .6 h+$ & Chr01:57218414-57219389(-) & AT4G17080 & $\begin{array}{l}\text { Histone H3 K4-specific methyltransferase SET7/9 family } \\
\text { protein }\end{array}$ \\
\hline Sobic.007G051800 & -7.39 & $36 h+v s .6 h+$ & Chr07:5299967-5302054(-) & AT5G57150 & $\begin{array}{l}\text { basic helix-loop-helix (bHLH) DNA-binding superfamily } \\
\text { protein }\end{array}$ \\
\hline Sobic.003G204900 & -7.27 & $48 h+v s .6 h+$ & Chr03:53476572-53477167(-) & [no close hit] & \\
\hline Sobic.new.1257 & -7.22 & $36 h+v s .6 h+$ & Chr01:15911715-15914760(+) & [no close hit] & \\
\hline Sobic.new.15945 & -7.21 & 72h-vs.6h- & Chr07:59702973-59707074(+) & [no close hit] & \\
\hline Sobic.004G340200 & -6.94 & $24 h+v s .6 h+$ & Chr04:67204166-67206072(+) & CCR1 & cinnamoyl coa reductase 1 \\
\hline Sobic.001G114300 & -6.85 & 72h-vs.6h- & Chr01:8956873-8957649(-) & [no close hit] & \\
\hline Sobic.new.15944 & -6.77 & $48 h+v s .6 h+$ & Chr07:59694635-59698364(-) & AT1G24090 & RNase $\mathrm{H}$ family protein \\
\hline Sobic.new.17185 & -6.77 & $36 h+v s .6 h+$ & Chr08:56526668-56529426(-) & [not protein coding] & \\
\hline Sobic.003G337400 & -6.64 & $72 \mathrm{~h}+\mathrm{vs} .6 \mathrm{~h}+$ & Chr03:66049426-66052170(-) & $\mathrm{C} 4 \mathrm{H}$ & cinnamate-4-hydroxylase \\
\hline Sobic.new.11126 & -6.64 & $36 h+v s .6 h+$ & Chr04:59772773-59773171(.) & [not protein coding] & \\
\hline Sobic.new.20531 & -6.62 & $36 h+v s .6 h+$ & Chr10:55587557-55589113(-) & [not protein coding] & \\
\hline Sobic.new.11575 & -6.55 & 72h-vs.6h- & Chr04:65102877-65103536(-) & [not protein coding] & \\
\hline Sobic.004G258500 & -6.55 & $24 h+v s .6 h+$ & Chr04:60408405-60410179(-) & AT1G12030 & Protein of unknown function (DUF506) \\
\hline Sobic.003G156200 & -6.53 & $72 \mathrm{~h}+\mathrm{vs} .6 \mathrm{~h}+$ & Chr03:17202371-17205169(-) & CYP709B2 & cytochrome P450, family 709 , subfamily B, polypeptide 2 \\
\hline Sobic.003G204750 & -6.50 & $48 \mathrm{~h}+\mathrm{vs} .6 \mathrm{~h}+$ & Chr03:53467868-53473076(+) & [no close hit] & \\
\hline Sobic.007G061300 & -6.45 & 72h-vs.6h- & Chr07:6435536-6437699(-) & [no close hit] & \\
\hline Sobic.002G391700 & -6.34 & $48 \mathrm{~h}+\mathrm{vs} .6 \mathrm{~h}+$ & Chr02:74441122-74442505(-) & AT4G11290 & Peroxidase superfamily protein \\
\hline
\end{tabular}




\begin{tabular}{|c|c|c|c|c|c|}
\hline Sobic.005G185600 & -6.18 & $72 \mathrm{~h}+\mathrm{vs} .6 \mathrm{~h}+$ & Chr05:67024041-67025037(+) & [no close hit] & \\
\hline Sobic.004G022900 & -6.05 & $24 h+v s .6 h+$ & Chr04:1812698-1815480(+) & AT1G74790 & catalytics \\
\hline Sobic.001G259600 & -5.33 & $24 \mathrm{~h}+\mathrm{vs} .6 \mathrm{~h}+$ & Chr01:31327155-31328316(+) & JAZ11 & jasmonate-zim-domain protein 11 \\
\hline Sobic.009G143500 & -4.81 & $12 \mathrm{~h}-\mathrm{vs} .6 \mathrm{~h}-$ & Chr09:50086875-50090678(-) & AT1G21460 & Nodulin MtN3 family protein \\
\hline Sobic.009G172500 & -4.32 & 12h-vs.6h- & Chr09:52753450-52757465(-) & PYD4 & PYRIMIDINE 4 \\
\hline Sobic.001G406800 & -4.31 & $12 \mathrm{~h}+\mathrm{vs} .6 \mathrm{~h}+$ & Chr01:69116259-69120681(+) & ASN1 & glutamine-dependent asparagine synthase 1 \\
\hline Sobic.002G401400 & -4.10 & 12h-vs.6h- & Chr02:75190443-75191454(-) & AT2G28420 & Lactoylglutathione lyase / glyoxalase I family protein \\
\hline Sobic.002G309000 & -3.84 & $12 \mathrm{~h}+\mathrm{vs} .6 \mathrm{~h}+$ & Chr02:68330828-68333547(+) & AT2G36170 & Ubiquitin supergroup;Ribosomal protein $\mathrm{L} 40 \mathrm{e}$ \\
\hline Sobic.001G412700 & -3.53 & $12 \mathrm{~h}-\mathrm{vs} .6 \mathrm{~h}-$ & Chr01:69506270-69508628(+) & GSTL2 & glutathione transferase lambda 2 \\
\hline Sobic.004G171400 & -3.51 & 12h-vs.6h- & Chr04:52396486-52397755(+) & GSTF13 & Glutathione S-transferase family protein \\
\hline Sobic.001G432800 & -3.50 & $12 \mathrm{~h}-\mathrm{vs} .6 \mathrm{~h}-$ & Chr01:71152961-71158082(+) & PEPCK & phosphoenolpyruvate carboxykinase 1 \\
\hline Sobic.003G284200 & -3.49 & $12 \mathrm{~h}+\mathrm{vs} .6 \mathrm{~h}+$ & Chr03:61812323-61814742(-) & CPNIFS & chloroplastic NIFS-like cysteine desulfurase \\
\hline Sobic.001G106600 & -3.35 & $12 \mathrm{~h}-\mathrm{vs} .6 \mathrm{~h}-$ & Chr01:8225098-8231079(+) & AT2G36630 & Sulfite exporter TauE/SafE family protein \\
\hline
\end{tabular}


Table 2.4: Summary of differentially expressed genes in cross-condition comparisons

\begin{tabular}{|l|l|l|l|}
\hline Comparison & $\begin{array}{l}\text { Genes differentially } \\
\text { expressed greater } \\
\text { than 2-fold }\end{array}$ & Upregulated & Downregulated \\
\hline $6 h+. v s .6 h-$ & 663 & 468 & 195 \\
\hline $12 h+. v s .12 h-$ & 797 & 520 & 277 \\
\hline $24 h+. v s .24 h-$ & 1,305 & 766 & 539 \\
\hline $36 h+. v s .36 h-$ & 2,006 & 992 & 1,014 \\
\hline $48 h+. v s .48 h-$ & 2,340 & 901 & 1,439 \\
\hline $72 h+. v s .72 h-$ & 3,564 & 1,321 & 2,243 \\
\hline All cross-condition & 4,818 & 2,118 & 2,810 \\
\hline
\end{tabular}


Table 2.5: Most highly upregulated genes in contrasts made across conditions.

\begin{tabular}{|c|c|c|c|c|c|}
\hline Gene ID & $\begin{array}{l}\text { peak } \\
\log _{2} \text { (fold } \\
\text { change) }\end{array}$ & $\begin{array}{l}\text { contrast } \\
\text { with peak } \\
\text { log2(fold } \\
\text { change) }\end{array}$ & chromosomal coordinates & $\begin{array}{l}\text { closest match in Ara- } \\
\text { bidopsis }\end{array}$ & Description \\
\hline Sobic.new.2302 & 12.70 & $24 h+v s .24 h-$ & Chr01:64040358-64040907(-) & [no close hit] & \\
\hline Sobic.new.1563 & 11.67 & $24 h+v s .24 h-$ & Chr01:23217188-23226278(+) & AT2G05390 & putative retroelement pol polyprotein \\
\hline Sobic.010G052700 & 11.01 & $24 h+v s .24 h-$ & Chr10:4116012-4118068(-) & IAA27/PAP2 & phytochrome-associated protein 2 \\
\hline Sobic.004G014700 & 10.72 & $72 \mathrm{~h}+\mathrm{vs} .72 \mathrm{~h}-$ & Chr04:1189463-1190764(-) & [no close hit] & \\
\hline Sobic.003G306500 & 10.55 & $12 \mathrm{~h}+\mathrm{vs} .12 \mathrm{~h}-$ & Chr03:63587722-63591672(+) & GH3-2 & Auxin-responsive GH3 family protein \\
\hline Sobic.new.12453 & 9.81 & $72 \mathrm{~h}+\mathrm{vs} .72 \mathrm{~h}-$ & Chr05:20029357-20037834(+) & [not protein coding] & \\
\hline Sobic.003G169300 & 9.44 & $24 h+v s .24 h-$ & Chr03:28855955-28856665(-) & ATCG00160 & ribosomal protein S2 \\
\hline Sobic.009G179000 & 9.16 & $72 \mathrm{~h}+\mathrm{vs} .72 \mathrm{~h}-$ & Chr09:53361509-53366268(+) & AT5G21090 & Leucine-rich repeat (LRR) family protein \\
\hline Sobic.new.7548 & 9.14 & $24 h+v s .24 h-$ & Chr03:38413317-38413592(.) & [not protein coding] & \\
\hline Sobic.004G333000 & 9.11 & $36 h+v s .36 h-$ & Chr04:66557869-66558715(+) & [no close hit] & \\
\hline Sobic.new.7546 & 9.00 & $24 h+v s .24 h-$ & Chr03:38376749-38377554(.) & [not protein coding] & \\
\hline Sobic.009G140150 & 8.92 & $72 \mathrm{~h}+\mathrm{vs} .72 \mathrm{~h}-$ & Chr09:49705378-49711110(-) & MLO1 & Seven transmembrane MLO family protein \\
\hline Sobic.002G253000 & 8.81 & $36 h+v s .36 h-$ & Chr02:63933586-63937861(-) & NAC038 & NAC domain containing protein 38 \\
\hline Sobic.002G234500 & 8.79 & $48 \mathrm{~h}+\mathrm{vs} .48 \mathrm{~h}-$ & Chr02:62562691-62563794(+) & AT4G30380 & Barwin-related endoglucanase \\
\hline Sobic.010G200600 & 8.50 & $36 h+v s .36 h-$ & Chr10:54360187-54363927(+) & AT3G07195 & RPM1-interacting protein 4 (RIN4) family protein \\
\hline Sobic.new.15944 & 8.50 & $12 \mathrm{~h}+\mathrm{vs} .12 \mathrm{~h}-$ & Chr07:59694635-59698364(-) & AT1G24090 & RNase $\mathrm{H}$ family protein \\
\hline Sobic.010G091700 & 8.45 & $12 \mathrm{~h}+\mathrm{vs} .12 \mathrm{~h}-$ & Chr10:8099384-8100834(+) & KDR & $\begin{array}{l}\text { basic helix-loop-helix (bHLH) DNA-binding superfamily } \\
\text { protein }\end{array}$ \\
\hline Sobic.002G285600 & 8.42 & $6 h+v s .6 h-$ & Chr02:66553976-66555074(+) & AT1G29500 & SAUR-like auxin-responsive protein family \\
\hline Sobic.new.15293 & 8.33 & $12 \mathrm{~h}+\mathrm{vs} .12 \mathrm{~h}-$ & Chr07:6495417-6497005(.) & AT3G39100 & \\
\hline Sobic.new.16742 & 8.30 & $24 h+v s .24 h-$ & Chr08:7233967-7234182(.) & [not protein coding] & \\
\hline Sobic.001G535900 & 8.27 & $36 h+v s .36 h-$ & Chr01:79929261-79930715(-) & TIP1;3 & tonoplast intrinsic protein $1 ; 3$ \\
\hline Sobic.004G160500 & 8.26 & $48 h+v s .48 h-$ & Chr04:50769266-50770262(+) & [no close hit] & \\
\hline Sobic.new.15945 & 8.21 & $72 \mathrm{~h}+\mathrm{vs} .72 \mathrm{~h}-$ & Chr07:59702973-59707074(+) & [not protein coding] & \\
\hline
\end{tabular}




\begin{tabular}{|c|c|c|c|c|c|}
\hline Sobic.new.12700 & 8.08 & $24 \mathrm{~h}+\mathrm{vs} .24 \mathrm{~h}-$ & Chr05:60428678-60428957(.) & [not protein coding] & \\
\hline Sobic.003G015700 & 8.00 & $36 \mathrm{~h}+\mathrm{vs} .36 \mathrm{~h}-$ & Chr03:1426081-1429733(+) & GH9C3 & glycosyl hydrolase 9C3 \\
\hline Sobic.001G224300 & 7.93 & $12 \mathrm{~h}+\mathrm{vs} .12 \mathrm{~h}-$ & Chr01:21485582-21489706(-) & CESA4 & cellulose synthase A4 \\
\hline Sobic.009G056600 & 7.81 & $48 \mathrm{~h}+\mathrm{vs} .48 \mathrm{~h}-$ & Chr09:5744548-5745144(-) & AT4G40070 & RING/U-box superfamily protein \\
\hline Sobic.005G030500 & 7.79 & $6 h+v s .6 h-$ & Chr05:2740887-2743334(+) & ARR8 & response regulator 3 \\
\hline Sobic.001G184800 & 7.77 & $6 \mathrm{~h}+\mathrm{vs} .6 \mathrm{~h}-$ & Chr01:15797997-15798792(+) & AT1G29500 & SAUR-like auxin-responsive protein family \\
\hline Sobic.004G336500 & 7.68 & $12 \mathrm{~h}+\mathrm{vs} .12 \mathrm{~h}-$ & Chr04:66846803-66847970(+) & IAA12/BDL & AUX/IAA transcriptional regulator family protein \\
\hline Sobic.007G172100 & 7.67 & $72 \mathrm{~h}+\mathrm{vs} .72 \mathrm{~h}-$ & Chr07:60704882-60706480(-) & AT5G50260 & Cysteine proteinases superfamily protein \\
\hline Sobic.002G285450 & 7.66 & $12 \mathrm{~h}+\mathrm{vs} .12 \mathrm{~h}-$ & Chr02:66550991-66551778(-) & AT1G29500 & SAUR-like auxin-responsive protein family \\
\hline Sobic.002G273400 & 7.53 & $48 \mathrm{~h}+\mathrm{vs} .48 \mathrm{~h}-$ & Chr02:65650640-65652688(+) & IAA4 & AUX/IAA transcriptional regulator family protein \\
\hline Sobic.009G006500 & 7.26 & $72 \mathrm{~h}+v s .72 \mathrm{~h}-$ & Chr09:563020-565136(-) & AT5G64700 & nodulin MtN21 /EamA-like transporter family protein \\
\hline Sobic.001G331200 & 6.52 & $6 h+v s .6 h-$ & Chr01:61899347-61901912(-) & GH3-2 & Auxin-responsive GH3 family protein \\
\hline Sobic.007G129201 & 6.38 & $6 h+v s .6 h-$ & Chr07:54571909-54573717(+) & AT5G62900 & \\
\hline
\end{tabular}


Table 2.6: Most highly downregulated genes in contrasts made across conditions.

\begin{tabular}{|c|c|c|c|c|c|}
\hline Gene ID & $\begin{array}{l}\text { peak } \\
\log _{2}(\text { fold } \\
\text { change) }\end{array}$ & $\begin{array}{l}\text { contrast } \\
\text { with peak } \\
\text { log2(fold } \\
\text { change) }\end{array}$ & chromosomal coordinates & $\begin{array}{l}\text { closest match in Ar- } \\
\text { abidopsis }\end{array}$ & Description \\
\hline Sobic.new.5133 & -14.25 & $12 \mathrm{~h}+\mathrm{vs} .12 \mathrm{~h}-$ & Chr02:62452730-62453844(.) & [no close hit] & \\
\hline Sobic.new.230 & -12.70 & $24 h+v s .24 h-$ & Chr01:2368735-2370876(+) & [not protein coding] & \\
\hline Sobic.004G292900 & -12.54 & $48 h+v s .48 h-$ & Chr04:63330856-63331736(-) & AT2G45180 & $\begin{array}{l}\text { Bifunctional inhibitor/lipid-transfer protein/seed storage } \\
2 \mathrm{~S} \text { albumin superfamily protein }\end{array}$ \\
\hline Sobic.002G122400 & -12.17 & $72 h+v s .72 h-$ & Chr02:16269204-16273011(+) & GLTP1 & glycolipid transfer protein 1 \\
\hline Sobic.010G234500 & -11.67 & $72 h+v s .72 h-$ & Chr10:57725721-57727469(-) & AT4G35160 & O-methyltransferase family protein \\
\hline Sobic.006G010301 & -11.47 & $72 \mathrm{~h}+\mathrm{vs} .72 \mathrm{~h}-$ & Chr06:1517753-1520636(-) & AT1G31950 & $\begin{array}{l}\text { Terpenoid cyclases/Protein prenyltransferases superfam- } \\
\text { ily protein }\end{array}$ \\
\hline Sobic.005G049600 & -11.21 & $48 \mathrm{~h}+\mathrm{vs} .48 \mathrm{~h}-$ & Chr05:4708347-4713388(-) & WAK5 & wall associated kinase 5 \\
\hline Sobic.001G231900 & -11.15 & $48 \mathrm{~h}+\mathrm{vs} .48 \mathrm{~h}-$ & Chr01:22434965-22435861(-) & [no close hit] & \\
\hline Sobic.007G090472 & -11.05 & $72 \mathrm{~h}+\mathrm{vs} .72 \mathrm{~h}-$ & Chr07:14128551-14165426(+) & CAS1 & cycloartenol synthase 1 \\
\hline Sobic.006G010100 & -10.91 & $72 \mathrm{~h}+\mathrm{vs} .72 \mathrm{~h}-$ & Chr06:1473844-1478555(-) & TPS21 & terpene synthase 21 \\
\hline Sobic.003G082900 & -10.60 & $72 h+v s .72 h-$ & Chr03:7101247-7103516(-) & $\mathrm{HCT}$ & $\begin{array}{l}\text { hydroxycinnamoyl-CoA shikimate/quinate hy- } \\
\text { droxycinnamoyl transferase }\end{array}$ \\
\hline Sobic.004G328601 & -10.50 & $72 \mathrm{~h}+\mathrm{vs} .72 \mathrm{~h}-$ & Chr04:66289147-66290070(-) & AT4G13180 & NAD(P)-binding Rossmann-fold superfamily protein \\
\hline Sobic.new.1275 & -10.50 & $24 h+v s .24 h-$ & Chr01:16750728-16750971(.) & [not protein coding] & \\
\hline Sobic.004G009300 & -10.10 & $72 \mathrm{~h}+\mathrm{vs} .72 \mathrm{~h}-$ & Chr04:793657-795297(-) & [no close hit] & \\
\hline Sobic.006G079300 & -9.87 & $72 \mathrm{~h}+\mathrm{vs} .72 \mathrm{~h}-$ & Chr06:44550695-44552325(+) & AT5G66390 & Peroxidase superfamily protein \\
\hline Sobic.005G086600 & -9.79 & $48 \mathrm{~h}+\mathrm{vs} .48 \mathrm{~h}-$ & Chr05:12030995-12032795(-) & OMT1 & O-methyltransferase 1 \\
\hline Sobic.006G170600 & -9.78 & $48 \mathrm{~h}+\mathrm{vs} .48 \mathrm{~h}-$ & Chr06:52722392-52723580(-) & TIP2;3 & tonoplast intrinsic protein $2 ; 3$ \\
\hline Sobic.006G172500 & -9.68 & $48 \mathrm{~h}+\mathrm{vs} .48 \mathrm{~h}-$ & Chr06:52842536-52843272(+) & AT4G12520 & $\begin{array}{l}\text { Bifunctional inhibitor/lipid-transfer protein/seed storage } \\
2 \mathrm{~S} \text { albumin superfamily protein }\end{array}$ \\
\hline Sobic.new.15522 & -9.58 & $24 h+v s .24 h-$ & Chr07:17952432-17952947(+) & [not protein coding] & \\
\hline Sobic.003G059000 & -9.14 & $48 \mathrm{~h}+\mathrm{vs} .48 \mathrm{~h}-$ & Chr03:5185672-5186593(+) & AT5G05500 & Pollen Ole e 1 allergen and extensin family protein \\
\hline Sobic.new.1245 & -8.77 & $36 h+v s .36 h-$ & Chr01:15401556-15402674(.) & OMT1 & O-methyltransferase 1 \\
\hline
\end{tabular}




\begin{tabular}{|c|c|c|c|c|c|}
\hline Sobic.004G000700 & -8.75 & $36 \mathrm{~h}+\mathrm{vs} .36 \mathrm{~h}-$ & Chr04:71139-72677(+) & LDOX & leucoanthocyanidin dioxygenase \\
\hline Sobic.002G278700 & -8.74 & $24 \mathrm{~h}+\mathrm{vs} .24 \mathrm{~h}-$ & Chr02:66066399-66068719(-) & AT1G14220 & Ribonuclease T2 family protein \\
\hline Sobic.009G181700 & -8.71 & $36 \mathrm{~h}+\mathrm{vs} .36 \mathrm{~h}-$ & Chr09:53557842-53560009(+) & AT5G07050 & nodulin MtN21 /EamA-like transporter family protein \\
\hline Sobic.new.1971 & -8.32 & $36 \mathrm{~h}+\mathrm{vs} .36 \mathrm{~h}-$ & Chr01:57218414-57219389(-) & AT4G17080 & $\begin{array}{l}\text { Histone H3 K4-specific methyltransferase SET7/9 family } \\
\text { protein }\end{array}$ \\
\hline Sobic.002G278800 & -8.16 & $24 \mathrm{~h}+\mathrm{vs} .24 \mathrm{~h}-$ & Chr02:66074257-66076549(-) & AT1G14220 & Ribonuclease T2 family protein \\
\hline Sobic.006G277700 & -8.10 & $36 \mathrm{~h}+\mathrm{vs} .36 \mathrm{~h}-$ & Chr06:60850342-60851907(-) & AT1G71695 & Peroxidase superfamily protein \\
\hline Sobic.008G080400 & -7.66 & $36 h+v s .36 h-$ & Chr08:13754078-13757147(-) & BGLU16 & beta glucosidase 16 \\
\hline Sobic.009G115500 & -5.89 & $6 \mathrm{~h}+\mathrm{vs} .6 \mathrm{~h}-$ & Chr09:45700110-45701391(+) & [no close hit] & \\
\hline Sobic.001G508700 & -5.84 & $6 h+v s .6 h-$ & Chr01:77663485-77666652(-) & AT5G45540 & Protein of unknown function (DUF594) \\
\hline Sobic.003G397300 & -5.60 & $24 \mathrm{~h}+\mathrm{vs} .24 \mathrm{~h}-$ & Chr03:70710569-70711410(-) & [no close hit] & \\
\hline Sobic.010G015800 & -5.59 & $6 \mathrm{~h}+\mathrm{vs} .6 \mathrm{~h}-$ & Chr10:1261319-1264806(+) & BB2 & RING/U-box superfamily protein \\
\hline Sobic.new.1757 & -5.01 & $6 \mathrm{~h}+\mathrm{vs} .6 \mathrm{~h}-$ & Chr01:39110625-39110947(.) & [not protein coding] & \\
\hline Sobic.002G418500 & -4.95 & $6 h+v s .6 h-$ & Chr02:76629585-76632298(-) & WRKY46 & WRKY DNA-binding protein 46 \\
\hline Sobic.004G002700 & -4.89 & $12 \mathrm{~h}+\mathrm{vs} .12 \mathrm{~h}-$ & Chr04:207244-208059(-) & AT5G46960 & $\begin{array}{l}\text { Plant invertase/pectin methylesterase inhibitor superfam- } \\
\text { ily protein }\end{array}$ \\
\hline Sobic.002G202800 & -4.68 & $6 h+v s .6 h-$ & Chr02:59275303-59277597(-) & WRKY40 & WRKY DNA-binding protein 40 \\
\hline Sobic.004G278100 & -4.65 & $6 \mathrm{~h}+\mathrm{vs} .6 \mathrm{~h}-$ & Chr04:62107160-62109382(+) & AT4G17670 & Protein of unknown function (DUF581) \\
\hline Sobic.008G002700 & -4.41 & $12 \mathrm{~h}+\mathrm{vs} .12 \mathrm{~h}-$ & Chr08:245146-246555(-) & CCR3 & CRINKLY4 related 3 \\
\hline Sobic.005G097800 & -4.35 & $12 \mathrm{~h}+\mathrm{vs} .12 \mathrm{~h}-$ & Chr05:15761595-15762827(-) & AT2G03200 & Eukaryotic aspartyl protease family protein \\
\hline Sobic.002G327700 & -4.26 & $12 \mathrm{~h}+\mathrm{vs} .12 \mathrm{~h}-$ & Chr02:69720169-69726258(-) & CRK23 & cysteine-rich RLK (RECEPTOR-like protein kinase) 23 \\
\hline
\end{tabular}




\section{Chapter 3}

\section{Investigation of host response in the Agrobacterium-plant interaction us- ing RNA-seq}

Note: This chapter includes re-analysis of RNA-seq data described in the following publication:

Duan K., Willig C.J., De Tar J.R., Spollen W.G., Zhang Z.J. (2017) Transcriptomic analysis of Arabidopsis seedlings in response to Agrobacterium-mediated transformation process. Molecular Plant-Microbe Interactions, 31:445-459. https://doi.org/10.1094/MPMI-10-17-0249-R

K. Duan performed the Agrobacterium inoculation experiments, collected seedling samples, performed RNA extraction sequencing submission, and carried out qRT-PCR experiments to validate RNA-seq data. All other work discussed in this chapter was performed by the author. 


\section{Summary}

Agrobacterium tumefaciens is a plant pathogen that can horizontally transmit DNA sequences into the genomes of eukaryotes. By attenuating the pathogenicity of A.tumefaciens, researchers have been able to manipulate this naturally-occurring phenomenon to accomplish genetic engineering of crop species. However, Agrobacteriummediated plant transformation is somewhat limited by host-range restriction of various A. tumefaciens strains and plant immune responses can limit the efficiency of stable transgene integration. To overcome these issues through technical advances, understanding the mechanisms by which vector and host genes interact to promote successful delivery and chromosomal incorporation of transgenes is essential. Though the identities and functional roles of Agrobacterium virulence genes in facilitating T-DNA translocation into plant cells are well-studied, the variety of host genes involved in this process are not. In this study, we performed whole transcriptome sequencing experiments on Arabidopsis thaliana whole seedlings treated with strains of Agrobacterium either able or unable to transfer T-DNA and virulence factors into plant cells, taking tissue samples throughout a time-course following seedling inoculation. In a re-analysis of data first reported in Duan et al. (2018), differential expression testing revealed over 2,500 genes differentially regulated by both Agrobacterium treatments relative to mock treatment. Co-expression clustering and gene set enrichment analysis enabled the examination of larger trends within the data. Genes upregulated by both Agrobacterium treatments included several associated with defense and stress responses (e.g., receptor-like kinases, heat-shock proteins, WRKY transcription factors) and those downregulated in both 
treatments included many involved in normal plant growth and metabolism (e.g., cell wall biogenesis enzymes, secondary metabolite biosynthetic enzymes, components of DNA replication). Relatively few genes showed differential expression responses between the two Agrobacterium treatments, but those that did pointed toward apparent inhibition of cytokinin signaling at multiple levels by the virulent Agrobacterium strain. This study provides multiple potential leads for future research on the genetics of Agrobacterium-plant interactions.

\section{Introduction}

Agrobacterium is a genus of gram-negative bacteria for which some members are capable of horizontal gene transfer to plants. For the past few decades since the first genetically engineered plants were reported in the early 1980s (Bevan et al. 1983), this naturally existing capability has been exploited by researchers and crop developers as a tool in the field of plant biotechnology for delivering genes of interest into target plants (i.e., plant transformation) for purposes such as genetic research or trait improvement. Other methods of accomplishing transformation in plants are available, such as bombardment of plant tissue with gold or tungsten microparticles bound to DNA. However, Agrobacterium-mediated transformation remains the most widely used gene delivery method in many plant species because as it has some distinct advantages over other systems including lower cost of consumables and equipment, ease of use, the ability to transfer long segments of DNA, and the production of higher rates of target plants retaining intact, single-copy transgenes following regeneration (Gao and Nielsen 2013, 
Mello-Farias and Chaves 2008). On the other hand, the breadth of application for Agrobacterium-mediated transformation is limited by factors such as host plant range and genotype specificity caused by differential immune response to Agrobacterium infection (Tzfira and Citovsky 2006).

The ecological role played by Agrobacterium species used in plant biotechnology (primarily A. tumefaciens and A. rhizogenes) is that of plant pathogens. A. tumefaciens strains possessing a tumor-inducing $(\mathrm{Ti})$ accessory plasmid are the causative agents of crown gall disease, which has been documented in over 600 plant species (De Cleene and De Ley 1976). Crown gall disease manifests in most cases as "tumor"-like growths that have altered metabolism forming on stems, branches, and roots of infected plants. Pathogenic strains of $A$. rhizogenes carry a distinct root-inducing (Ri) plasmid and give rise to hairy root disease, which involves proliferation of highly-branch adventitious root structures on the host near the infection site. Both species confer their respective diseases by transmitting a DNA segment located on the Ti/Ri plasmid into host plant cells where it can be integrated into the host's genome and plant-active genes can be expressed (Sinkar et al. 1987). This segment is termed the T-DNA. The T-DNA delivered to plant cells by wild-type $A$. tumefaciens, which is both more well-studied and more widely used in transformation than $A$. rhizogenes, contains multiple genes that induce the biosynthesis of the plant hormones auxin and cytokinin as well as opines, which are amino acid-sugar conjugates that Agrobacterium can metabolize as an energy source to the exclusion of other microbial species (Britton et al. 2008). Strains of $A$. tumefaciens have been developed for use in routine laboratory plant transformation which have 
been "disarmed" (i.e., made non-pathogenic) by having the disease-causing genes mutated or removed from their Ti plasmids (Torisky et al. 1997). Smaller, more manipulable plasmids that contain a gene or genes of interest flanked by boundary sequences that mimic those of the wild-type T-DNA are mobilized to these disarmed Agrobacterium strains, which can then be used to transmit a chosen sequence, rather than that giving rise to gall formation, into plant cells via the same underlying molecular mechanism (Hoekema et al. 1983).

The well-characterized infection process of $A$. tumefaciens is dependent on a cassette of genes known as the vir (virulence) regulon, which is also encoded on the $\mathrm{Ti}$ plasmid. Infection begins when Agrobacterium perceives a stress signal from a nearby plant in the form of phenolic compounds in the presence of sugars that may be released in reaction to plant wounding. This signal is relayed in the Agrobacterium cell by the constitutively expressed proteins VirA and VirG, which induce the transcription of all vir genes necessary for T-DNA transfer (Gelvin 2006). The pathogen undergoes attachment to the cell wall of the target plant, which depends on some bacterial virulence genes not encoded on the Ti plasmid (chvA and chvB) (Douglas et al. 1982). A set of protein molecules including those encoded by virB1-11 and virD4 participate in the construction of what is referred to as a type IV secretion system (T4SS), which forms a connection between the bacterial cell and the plant cell cytoplasm (Aguilar et al. 2011). The VirD1 and VirD2 proteins nick the T-DNA sequence at its outer boundaries (the right- and left-border sequences). The single-stranded T-DNA segment is then covalently attached at its $5^{\prime}$ end to a VirD2 molecule and is trafficked through the T4SS into the plant cell cytoplasm 
along with the proteins VirE2, VirE3, VirD5, and VirF. Upon entering the plant cell, the TDNA strand is coated along its length with multiple VirE2 molecules, thereby forming the mature "T-complex" (Lacroix and Citovsky 2019). VirD2 and VirE2 contain plant-active nuclear localization signals (NLS) within their peptide sequences, allowing the T-complex to pass through the nuclear envelope (Herrera-Estrella et al. 1990, Tzfira and Citovsky 2001). Within the nucleus, the T-DNA strand is uncoated as the VirE2 proteins are degraded by the plant's ubiquitin-mediated proteolysis system. The T-DNA may then be integrated into the plant genome, by way of a poorly-understood mechanism, at an apparently random position (Gelvin 2017).

Just as agrobacteria are able to perceive plant signals, plants can perceive the presence of pathogenic microbes and respond accordingly. One mechanism that allows for this is the recognition of pathogen-associated molecular patterns, or PAMPs, molecules or epitopes that often are evolutionarily conserved among groups which bind to plant transmembrane receptor protein and activate a common set of responses within the host cells that are referred to as PAMP-triggered immunity (PTI). The generalized PAMP response includes MAP kinase signaling activation, intracellular calcium flux, reactive oxygen species (ROS) burst, callose deposition in affected tissues, and induction of defense-related genes, all of which work to restrict subsequent infection by the pathogen (Zhang and Zhou 2010, Zipfel 2009). The most well-known bacterial PAMP perceived by plants is flg22, an epitope derived from bacterial flagellin, which is recognized by the receptor-like kinase protein FLAGELLIN-SENSING 2 (FLS2) in Arabidopsis thaliana (Chinchilla et al. 2006). However, a peptide sequence divergence in the Agrobacterium 
flg22 region allows it to evade detection by this mechanism in multiple plant species (Bauer et al. 2001). The Agrobacterium-derived PAMP elongation factor Tu (EF-Tu; or more specifically, the epitope elf18 within EF-Tu) however, is perceived by Arabidopsis via its receptor $E F R$ and mutants for this gene display higher transformation frequencies than wild-type plants (Zipfel et al. 2006). Other bacterial PAMPs that may affect the $\mathrm{Ag}$ robacterium-plant interaction include lipopolysaccharide (LPS) and peptidoglycan (PGN) (Gust et al. 2007, Livaja et al. 2008).

Some plant pathogens (e.g., Pseudomonas syringae) have evolved mechanisms which serve to counter PTI by disrupting the signaling pathway at some key node, often through the action of protein molecules secreted into plant cytoplasm, which are termed "effectors". Plants, in turn, have evolved very specific detection mechanisms for many of these effector proteins, which often result in a much stronger defense response, called effector-triggered immunity (ETI). These mechanisms tend to (though not always) function in race-specific pathogen resistance, in contrast to the more general, but weaker, PTI (Gassmann and Bhattacharjee 2012). Though there is no evidence for ETI or even for pathogen-derived genes functioning as effectors in the Agrobacteriumplant interaction (Hwang et al. 2015), it is conceivable that certain transferred factors from Agrobacterium could promote the efficiency of the transformation process by dampening the initial PTI response. Thus, to investigate this possibility we used whole transcriptome sequencing to compare gene expression in plants that were inoculated with either of two disarmed Agrobacterium strains, both capable of inciting PTI, that differ in their ability to form a T4SS and to transfer T-DNA and the translocated Vir proteins 
into plant cells, against plants that received an Agrobacterium-free mock treatment. An enhanced understanding of how Agrobacterium ensures that T-DNA is successfully delivered into plant cells could inform the development of techniques that will expand transformation capabilities in the field of plant biotechnology.

\section{Results}

RNA-seq sample collection and read alignment

This study utilized the raw RNA-seq data associated with the publication Duan et al. (2018). 10-day old seedling of Arabidopsis thaliana were inoculated with either of two strains of Agrobacterium tumefaciens (A136 or At804) or received mock treatment according to the AGROBEST protocol (Wu et al. 2014). Though both of these strains, which are described in Veena et al. (2003), are "disarmed," having lost the ability to induce tumor formation in plants, they differ in their ability to transmit molecular factors into plant cells. Strain A136 is deficient in transfer of Vir proteins and T-DNA (it contains no Ti plasmid) and is thus referred to hereafter as the "avirulent" strain. At804 contains a super-virulent Ti plasmid as well as a binary plasmid with a T-DNA sequence containing a GUS-intron gene, a visual marker expressible only in plant cells. Thus, At804 is referred to as the "virulent" strain. Samples of Arabidopsis seedlings were collected at timepoints of $0,3,6,12,24$, and 48 -hours post-inoculation in each parallel treatment condition for total RNA extraction and subsequent Illumina sequencing to assess the differential effect of infection by each strain on host gene expression using mock treatment as a 
negative control. Two biological replicates were submitted for sequencing per treatment/time-point combination, resulting in 32 total samples.

Summary pre-processing, alignment, and gene assignment data for all samples is displayed in Table 3.1. Samples from the first biological replicate were sequenced at an average depth of 8.5 million reads per sample, whereas the second replicate averaged 33.5 million per sample (nearly 4 times greater), resulting in a total raw read count of 671 million and an overall average per-sample read count of 21 million. Trimming of raw reads led to $3.52 \%$ of the total raw reads being filtered out. Removal of sequences aligning to Arabidopsis ribosomal and mitochondrial DNA sequences led to a further $0.61 \%$ reduction in total reads. The remaining cleaned read sets were aligned to the Araport 11 Arabidopsis thaliana genome assembly (Cheng et al. 2017), resulting in a 96.4\% unique mapping rate. $96.8 \%$ of these uniquely mapped reads were assigned to a gene locus as annotated by the GFF3 file provided with the Araport 11 assembly. Overall, $89.3 \%$ of raw reads contributed to the per-gene read counts used for differential expression analysis.

\section{Differential expression analysis}

A table containing per-gene read count values for each sample was constructed to be used in differential expression analysis. A significant batch effect separating samples from each of the two replicates was anticipated due to differences in library size and the time that the samples were originally collected. Thus, a statistical batch correction was attempted prior to moving forward with differential expression analysis. The $\mathrm{R}$ 
package ComBat-seq (Zhang et al. 2020) was employed to adjust the data using a negative binomial regression model while retaining integer read count values. See Figure 3.1 for plots displaying the effect of batch correction on the principal component distribution between the two replicates. Three-dimensional scatterplots were generated to visualize the relative expression of genes between the different treatments at each timepoint. These are shown in Figure 3.2. The batch-adjusted read count table was then used to perform differential expression analysis using DESeq2 (Love et al. 2014). Samples were grouped by treatment, time-point, and replicate with treatment being used as the primary factor in the experimental model. Low-abundance genes (fewer than 10 total read counts) were excluded from analysis, resulting in 4,743 of the original set of 27,655 annotated Arabidopsis genes (17.2\%) being dropped. The Wald's test function of DESeq2 was employed to contrast gene expression in each Agrobacterium treatment sample to the mock treatment sample at each corresponding time-point. Contrasts were also made between each sample treated with the virulent strain and samples treated with the avirulent strain at each time-point.

Genes that showed at least a 2-fold difference in expression for which the false discovery rate was below 0.05 were considered as differentially expressed in a given contrast. Venn diagrams showing numbers of genes common and unique to sets of differentially expressed genes in various contrasts are displayed in Figure 3.3. In the avirulent treatment vs. mock comparisons, 2,902 unique genes were differentially expressed in one or more contrasts $(1,405$ of these were upregulated and 1,497 were downregulated). 8 genes were called as differentially expressed at the 3-hour time-point, all of 
which were upregulated, and by the 48-hour time-point 2,579 genes were differentially expressed (1,200 upregulated and 1,379 downregulated). 6 genes were upregulated in all five of the contrasts, 56 were upregulated in contrasts at four time-points, and 106 were upregulated at three timepoints. No genes were downregulated in all five contrasts, however, 18 were downregulated in four contrasts, and 97 were downregulated in three contrasts. In the virulent treatment vs. mock comparisons, 4,648 genes were differentially expressed, of which 2,133 were upregulated and 2,515 were downregulated. 16 genes were called as differentially expressed at the 3-hour time-point, only 1 of which was downregulated. At the 48-hour time-point, 4,400 were differentially expressed (2,000 upregulated and 2,400 downregulated). 8 genes were upregulated in all five contrasts while none were downregulated in all contrasts. 50 genes were upregulated and 29 genes were downregulated in four of the five contrasts. 130 genes were upregulated and 153 were downregulated in three of the five contrasts. 893 total genes were upregulated in at least one contrast in the virulent vs. mock comparison which were not upregulated in the avirulent vs. mock comparison, while 165 genes were upregulated only in the avirulent vs. mock comparison. For the virulent vs. avirulent comparison, very few genes were significantly differentially expressed. 4 genes were consistently upregulated at the 6,12, and 24-hour time-points, with 1 of these also being upregulated as early as 3 hours. 2 genes were upregulated at the 48 -hour time-point, which were not seen in any other contrast. 1 and 2 genes were downregulated at the 24 and 48-hour time-points, respectively, but none were downregulated in more than one contrast. 
Highly differentially expressed genes

The most highly upregulated gene in the avirulent vs. mock comparison at the 3hour time-point was AT1G52560, annotated as coding for an HSP20-like chaperones superfamily protein $\left(\log _{2} \mathrm{FC}=5.53\right)$. In the virulent vs. mock comparison, the most highly upregulated gene at the earliest time-point $\left(\log _{2} \mathrm{FC}=7.61\right)$ was $A T 4 G 30380$, annotated as Barwin-related endoglucanase coding for the EXPANSIN-LIKE B2 protein, which is putatively involved in cell wall modification. Other genes that were among the most highly upregulated at 3 hours post-inoculation were AT5G39580 (a peroxidase superfamily protein), WRKY family transcription factor 22 (WRKY22), and AT4G27450, annotated as coding for an aluminum-induced protein with YGL and LRDR motifs. The most highly upregulated gene overall at any time-point was, for both the avirulent vs. mock and virulent vs. mock comparisons, PATHOGENESIS-RELATED GENE 1 (PR1) at the 6-hour timepoint in both cases ( $\log _{2} \mathrm{FC}=15.17$ and 15.03 , respectively) The set of genes that were most highly upregulated through all contrasts included several heat-shock proteins (AT1G52560, HSP17.6II, AT2G29500, HSP17.4, HSP17.6A, HSP22.0, HSP21, and AT1G53540) and genes related to pathogen response (MILDEW RESISTANCE LOCUS O 12 [MLO12], FLG22-induced receptor-like kinase 1 [FRK1] and Resistance Methylated Gene 1 [RMG1]). Other highly upregulated genes of note were a member of the AP2/ERF transcription factor family (AT1G22810), glutathione S-transferase TAU 11, and sucrose-proton symporter 7 (SUC7). 
There was a high degree of overlap in the top upregulated genes between the avirulent vs. mock and virulent vs. mock comparisons. Only 6 of the top upregulated genes in the virulent vs. mock comparison were not significantly upregulated in any avirulent vs. mock contrast. This set included the RING/U-box superfamily protein encoding ARIADNE 12 (ARI12) along with ARABIDOPSIS THALIANA RESPONSE REGULATORS 5 and 7 (ARR5 and ARR7), WALL ASSOCIATED KINASE 3 (WAK3), MPK3/6-targeted VQ motifcontaining protein 2 (MVQ2), and AT3G28570, coding for a P-loop containing ATPase protein. On the other hand, only 1 top upregulated gene in the avirulent vs. mock comparison (AT4G03450, an ankyrin repeat family protein) was not significantly upregulated in any virulent vs. mock contrast. Table 3.2 displays a list of genes that were among the top 10 most highly upregulated in each contrast along with their functional annotations. There were no genes called as significantly downregulated at the 3-hour timepoint in the avirulent vs. mock contrast, whereas a single gene coding for a boron transporter, REQUIRES HIGH BORON 2 (BOR2), was downregulated in the virulent vs. mock 3hour contrast $\left(\log _{2} \mathrm{FC}=-2.48\right)$. The most highly downregulated gene in the avirulent vs. mock 6-hours post-inoculation contrast was ROOT HAIR SPECIFIC 7 (RHS7/PRP1) at a $\log _{2}$-fold change of -7.49 , whereas the most downregulated gene at 6 hours in the virulent vs. mock comparison was AT5G04960, coding for a Plant invertase/pectin methylesterase inhibitor superfamily protein $\left(\log _{2} \mathrm{FC}=-5.05\right)$. Other genes that were strongly downregulated early in the time-course in response to Agrobacterium inoculation included ferric reduction oxidase 2 (FRO2), AT4G31470, which codes for a CAP superfamily protein, the UDP-Glycosyltransferase superfamily gene UGT72E, SKU5 similar 15 and 16 
(sks15 and sks16), and leucine-rich repeat/extensin 1 (LRX1). The most highly downregulated gene overall at any time-point was, for both the avirulent vs. mock and virulent vs. mock comparisons, nicotianamine synthase 2 (NAS2) at the 48-hour time-point in both comparisons $\left(\log _{2} \mathrm{FC}=-19.48\right.$ and -19.70 , respectively). Common gene descriptions that were observed among the set of highly downregulated genes included proline-rich and extensin-like proteins (AT5G10130, AT3G28550, AT4G08410, AT5G06630, AT3G54580, AT1G23720, EXT6, EXT10, EXT13), peroxidase superfamily proteins (AT1G30870, AT3G49960, AT3G01190, PRX56), late embryogenesis abundant-like proteins (AT5G60520, AT3G19430, AT5G54370), and various other genes related to carbohydrate metabolism/cell wall maintenance such as arabinogalactan protein 30 (AGP30), xyloglucan endotransglucosylase/hydrolase 12 (XTH12), expansin A18 (EXPA18), cellulose synthase-like B (CSLBO2), and beta glucosidase 34 and 35 (BGLU34 and BGLU35). Only 1 of the highly downregulated genes in the avirulent vs. mock comparison (AT1G49450, annotated as a member of the transducin/WD40 repeat-like superfamily) was not significantly downregulated at any time-point in the virulent vs. mock comparison. Table 3.3 displays genes that were among the top 10 most downregulated in each contrast along with functional annotations.

Clustering results and patterns 
Assignment of differentially expressed genes to co-expression clusters was performed using the python package clust. The clust method uses a pool of seed clusters generated by multiple rounds of $k$-means clustering using different $K$ values, followed by evaluation of these seed clusters by the $\mathrm{M}-\mathrm{N}$ scatter plots technique. This results in an optimal number of clusters with low dispersion values and distinct expression patterns, which is more repeatable and matches biological expectations better than other methods (Abu-Jamous and Kelly 2018). However, consequently not all genes in the input data set are assigned to a cluster. From an input set of 5,047 differentially expressed Arabidopsis thaliana genes, 1,847 genes were grouped into 12 clusters that ranged in size from 11 genes (cluster 4) to 422 (cluster 5). Clusters 2, 3, 4, 8, and 9 contained only genes that were downregulated in at least one contrast, whereas clusters $6,7,10,11$, and 12 contained only genes that were upregulated in at least one contrast. 329 genes grouped into cluster 1 were downregulated and 13 were upregulated. In cluster 5421 genes were downregulated and only 1 was upregulated. See Figure 3.4 for a summary line plot showing mean expression trends within the different clusters.

Some abundant gene descriptions observed with the clusters of downregulated genes included 22 genes in cluster 1 annotated as "Cysteine/Histidine-rich C1 domain family protein," 5 genes in cluster 1 annotated as "Integrase-type DNA-binding superfamily protein" (AP2/ERF family transcription factors), and 4 genes in cluster 2 annotated as expansins. Sets of gene descriptions among the upregulated cluster included 5 genes in cluster 5 annotated as "Core-2/I-branching beta-1,6-N-acetylglucosaminyltransferase family protein," 7 genes in cluster 5 annotated as "Transducin/WD40 repeat-like 
superfamily protein," 5 genes in cluster 6 annotated as "Tetratricopeptide repeat (TPR)like superfamily protein," 7 genes in cluster 10 annotated as "Disease resistance protein (TIR-NBS-LRR class) family," 4 genes in cluster 10 annotated as "DNAJ heat shock N-terminal domain-containing protein," and 12 genes in cluster 10 annotated as RNA binding proteins. There were also gene descriptions for which subgroups followed distinct expression patterns in both upregulated and downregulated clusters. For instance, there were 6 genes annotated as "alpha/beta-Hydrolases superfamily protein" in cluster 5, and 4 genes with this description in cluster 10. 5 genes annotated as "Homeodomainlike superfamily protein" were in cluster 6 and 3 were found in cluster 1 . For genes annotated as "Leucine-rich repeat protein kinase family protein," 9 were assigned to cluster 5, 5 were assigned to cluster 1 , and 5 were assigned to cluster 10. For "RING/U-box superfamily protein" genes, 17 were assigned to cluster 10,5 were assigned to cluster 6 , and 4 were assigned to cluster 5 .

Gene set enrichment analysis

The online gene functional annotation tool DAVID (Sherman and Lempicki 2009) was used to obtain lists of significantly enriched gene set categories for the differentially expressed genes at each time-course contrast made between the avirulent vs. mock and virulent vs. mock comparisons. Gene set categories used in this analysis included the Gene Ontology (GO) biological process (BP) and molecular function (MF) categories, the PIRSuperFamily (PIRSF) classification system, Simple Modular Architecture Research 
Tool (SMART) and InterPro protein domain categorization, and KEGG pathway classification. Only terms that were significantly overrepresented in at least one differentially expressed gene list were used for comparison. Manual inspection was used to reduce the list of enriched terms by eliminating those that were redundant or highly overlapping with another in the same gene set, those that had an adjusted $P$-value near the significance threshold in only one or two differentially expressed gene sets, or those that were of minimal relevance to the Agrobacterium-plant interaction. This resulted in a list of 36 enriched terms from the avirulent vs. mock comparison (21 among upregulated genes and 15 among downregulated genes) and 49 enriched terms in the virulent vs. mock comparison ( 28 from upregulated gene lists and 21 from downregulated). 11 of these terms were shared between the upregulated gene lists in each comparison, while 12 terms were shared between the downregulated gene lists in each comparison. Figure 3.5 shows the selected overrepresented gene sets for each contrast made through the time-course for both the avirulent vs. mock and virulent vs. mock contrasts.

The terms that were overrepresented among upregulated genes at the earliest points in time and remained significantly enriched through each contrast of the timecourse in the avirulence vs. mock comparison were the GO biological process term "response to heat" (GO:0009408), the InterPro classification "HSP20-like chaperone" (IPR008978), and the KEGG pathway "Protein processing in endoplasmic reticulum" (ath04141). These categories were also each overrepresented among upregulated genes in the virulence vs. mock comparisons, though beginning at the 6-hour time-point. Other categories that were among the most highly enriched among upregulated genes 
in both the avirulent vs. mock and virulent vs. mock comparisons included the GO biological process terms "response to chitin" (GO:0010200), "response to hydrogen peroxide" (GO:0042542), and "defense response to bacterium" (GO:0042742), the GO molecular function term "sequence-specific DNA binding" (GO:0043565), and the PIRSF term "Endochitinase" (PIRSF001060). Terms that were enriched among upregulated genes only in the avirulent vs. mock comparison were the KEGG pathway term "Plant-pathogen interaction" (ath04626) in the 6-hour contrast, the InterPro term "Lysozyme-like domain" (IPR023346) and the GO biological process term "amino sugar metabolic process" in the 6 and 12 hour contrasts, the KEGG pathway terms "Phenylalanine, tyrosine and tryptophan biosynthesis" (ath00400) and "Glutathione metabolism" (ath00480) and the InterPro term "Thioredoxin-like fold" (IPR012336) at the 24-hour contrast, and the GO biological process terms "lignin biosynthetic process" (GO:0009809) and "response to jasmonic acid" (GO:0009753) at the 24 and 48-hour contrasts. Terms enriched only in the upregulated gene lists of the virulent vs. mock comparison included the GO biological process terms "cell wall macromolecule catabolic process" (GO:0016998) and the InterPro term "FAD linked oxidase, N-terminal" (IPR006094) at the 12 and 24-hour contrasts, and the GO biological process terms "mRNA splicing, via spliceosome" (GO:0000398) and "protein phosphorylation" (GO:0006468), the GO molecular function terms "protein serine/threonine kinase activity" (GO:0004674) and "UDP-glucosyltransferase activity" (GO:0035251), the InterPro terms "RNA recognition motif domain" 
(IPR000504), “NB-ARC” (IPR002182), “DNA-binding WRKY” (IPR003657), and "Zinc finger, RING/FYVE/PHD-type" (IPR013083), and the SMART term "RRM" (SM00360) all at the 48-hour contrast.

Gene set terms enriched among downregulated genes in both the avirulent vs. mock and virulent vs. mock comparisons included the GO biological process terms "plant-type cell wall organization" (GO:0009664), "hydrogen peroxide catabolic process" (GO:0042744), "fatty acid biosynthetic process" (GO:0006633), and "DNA replication initiation" (GO:0006270), the molecular function terms "structural constituent of cell wall" (GO:0005199), "heme binding" (GO:0020037), and "guiding stereospecific synthesis activity" (GO:0042349), and the KEGG pathways "Phenylpropanoid biosynthesis" (ath00940) and "Biosynthesis of secondary metabolites" (ath01110). The terms that were specific to the downregulated gene lists of the avirulent vs. mock comparison were the GO biological process term "microtubule-based movement" (GO:0007018) and the molecular function terms "copper ion binding" (GO:0005507) and "cyclin-dependent protein serine/threonine kinase regulator activity" (GO:0016538). Terms that were enriched only among the downregulated gene lists in the virulent vs. mock comparison included the GO biological process terms "transmembrane receptor protein tyrosine kinase signaling pathway" (GO:0007169), "xyloglucan metabolic process" (GO:0010411), "response to endoplasmic reticulum stress" (GO:0034976), and "cellular response to nitric oxide" (GO:0071732) as well as the molecular function terms "hydrolase activity, hydrolyzing O-glycosyl compounds" (GO:0004553), "transferase activity, transferring glycosyl groups" (GO:0016757), and "transmembrane transporter activity" (GO:0022857). 
Differentially expressed transcription factor genes

Transcription factors comprised 7.9\% (111 out of 1,405) and 7.7\% (165 out of 2,133 ) of the genes upregulated in the avirulent vs. mock and virulent vs. mock comparisons, respectively, while among downregulated genes in each comparison, they made up $4.1 \%$ (62 out of 1,497) and 5.5\% (139 out of 2,515) of the total genes, respectively. 97 of the upregulated transcription factors were shared among the two comparisons, while 68 were exclusive to the virulent vs. mock comparison and 14 were exclusive to the avirulent vs. mock comparison. For the downregulated transcription factors, 56 were held in common between the comparisons, 83 were unique to the virulent vs. mock comparison, and 6 were unique to the avirulent vs. mock comparison. The transcription factor families with the highest numbers of differentially expressed members were AP2/ERF, bHLH, MYB, and WRKY. Other families with significant numbers of differentially expressed genes that were markedly higher among the upregulated gene lists included NAC, HSF, C3H, bZIP, Tri-helix, and Nin-like. Families with higher numbers of differentially expressed genes among downregulated lists included LBD and G2-like transcription factors. Figure 3.6 shows the most highly represented transcription factor families among sets of upregulated or downregulated genes for each comparison.

Notable transcription factor genes that were highly upregulated at multiple timepoints in both comparisons included AP2/ERF family members AT1G22810, ABR1, AT5G52020, DREB2A, and DREB2C, C2H2 factors RHL41 and ZF2, CZF1 (a member of the C3H family, the GRAS transcription factor SCARECROW-like 14, the HD-ZIP containing 
gene $A T H B 18$, heat shock transcription factors (HSF) HSFA2, HSFB2B, and HSFB2A, LOB domain-containing protein genes $\angle B D 40$ and $\angle B D 41$, MYB transcription factors MYB101 and MYB15, NAC domain containing protein genes NACO42/JUB1, NACO53, and ATAF2, Trihelix domain gene $A T 5 G 01380$, and the WRKY transcription factors WRKY22 and WRKY4O. Some transcription factors that were downregulated at multiple time-points in both comparisons were AP2/ERF transcription factors AT4G13620, LEP and thy (AT5G25810), the bHLH family genes FRU, AT3G07340 and B70, the G2-like transcription factor AT5G06800, the HD-ZIP gene HB20, and NAC domain containing protein NAC015/BEARSKIN1. Expression plots for select transcription factor genes upregulated and downregulated in both Agrobacterium treatment vs. mock comparisons are displayed in Figures 3.7 and 3.8, respectively.

Some differentially expressed genes were significantly upregulated or downregulated only in either the avirulent vs. mock or virulent vs. mock comparison and not in the other. The majority of these were only seen at the 48-hour contrast in either comparison, indicating that these may be only weakly differentially expressed and may therefore not represent genes that truly show a differential response to the two Agrobacterium treatments. However, a few of these genes were significantly differentially expressed at multiple time-points in one comparison, but not the other. The only transcription factor gene upregulated at multiple time-points that was specific to the avirulent vs. mock comparison was WRKY53 at the 12 and 24-hour contrasts, whereas NAC001 was upregulated specific to the virulent vs. mock comparison at the 12,24 , and 
48-hour contrasts. For downregulated transcription factors, none were differentially expressed at multiple time-points that were unique to the avirulent vs. mock comparison. However downregulated transcription factors specific to the virulent vs. mock comparison included MYB36, MYB48, PIL2, LRL3, bZIP3, MGP, AT5G22890, and NUC at the 24 and 48-hour contrasts, and WRKY65, MYB88, AT1G31050, and AGL12 at the 12, 24, and 48-hour contrasts.

Differentially expressed hormone-related genes

A list of Gene Ontology terms associated with metabolism, regulation, transport, signaling pathways of, and response to plant hormones was obtained using the online tool Quick GO (https://www.ebi.ac.uk/QuickGO/) (Binns et al. 2009). The list of GO IDs was then used to extract genes assigned to each category from a gene association dataset acquired from The Arabidopsis Information Resource (TAIR) (https://www.arabidopsis.org/download_files/GO_and_PO_Annotations/Gene_Ontology_Annotations/gene_association.tair.gz). Lists of genes associated with each category were then compared against lists of upregulated and downregulated genes in each comparison and the resulting lists were annotated with gene names and descriptions. These differentially expressed gene sets were then assessed for differences with respect to genes associated with each hormone type.

Notable differences with respect to auxin-related genes were that differentially expressed AUX/IAA transcriptional co-regulators were almost exclusively among the upregulated genes (with the exception of IAA28). The list of upregulated genes from this 
family included IAA1, 2, 5, 14, 16, 19, 30, and 34 . The AUXIN RESPONSE FACTOR gene ARF19 was also among those upregulated. Among the downregulated genes, 6 were involved in the polar transport of auxin (PIN7, AXR4, LAX3, AUX1, PIN5, EIR1), 6 genes were involved in auxin homeostasis, and 5 were involved in cellular response to auxin stimulus. YUC9 and YUC3, genes involved in the final biosynthetic step of the auxin IAA were among those downregulated. Interestingly, other genes involved in the auxin biosynthetic pathway were upregulated (e.g., ASA1, ASB1, AO1).

For ethylene, 7 genes involved in the ethylene biosynthetic process were upregulated and 2 were downregulated. 5 of the upregulated genes coded for 1-aminocyclopropane-1-carboxylate (ACC) synthase enzymes (ACS2, 4, 6, 7, and 8). Both of the 2 downregulated biosynthetic genes ( $\mathrm{ACO}$ and 5) coded for ACC oxidase enzymes, while none of the upregulated genes performed this function. ACO catalyzes the final step in ethylene production while ACC catalyzes the previous step. Thus, even though Agrobacterium treatment seems to have a differential effect on the expression of these two classes of enzymes, it is unclear what effect it has on ethylene biosynthesis overall. The number of genes involved in ethylene signaling and response were about the same between the upregulated and downregulated sets. With respect to gibberellins, the set of downregulated genes contained 7 that were involved in the response to gibberellin, 1 that played a role in gibberellic acid homeostasis, and 1 that was assigned to "cellular response to gibberellin stimulus." The upregulated gene set contained 3 genes (GA2OX2, 4, and 6) that were annotated as being involved in both gibberellin biosynthesis and catabolism as well as 8 genes that are responsive to gibberellin. The gene GIM2 
showed early and strong upregulation in both comparisons. This gene is known from the role it plays in seed germination through alteration of the gibberellic acid metabolic pathway in Arabidopsis (Xiong et al. 2018). Genes related to jasmonic acid also showed no clear difference between downregulated and upregulated genes. The jasmonate receptor COI1 was upregulated in both comparisons in the 48-hour contrast. However, the $J A Z$ signaling components were both among those upregulated $(J A Z 1,3$, and 5$)$ and downregulated (JAZ4 and 11). Genes involved in jasmonate catabolism (JOX2, JOX4, and $J R G 21)$ as well as biosynthesis (LOX4, OPR3, and SSI2) were upregulated and genes involved in biosynthesis ( $A O C 2$ and 4$)$ and metabolism of methyl jasmonate (MES1, 7, 11, 12, and 15) were downregulated. Expression plots for select differentially expressed hormone-related genes are displayed Figure 3.9.

Agrobacterium treatment showed a clear effect on metabolism and signaling of cytokinin, some of which was specific to treatment with the virulent strain. Among the downregulated genes related to cytokinin, are 3 involved in biosynthesis (IPT3, 5, and 7), 2 involved in cytokinin transport ( $A B C G 14$ and PUP1), and several involved in the cytokinin-activated signaling pathway such as WOL, AHK2, AHK5 and AHP1. The gene SOFL1, a positive regulator of cytokinin levels, was downregulated in the virulent vs. mock comparison at the 48-hour time-point. No biosynthesis or transport genes were seen among those that were upregulated. Instead, genes involved in cytokinin catabolism (CKX3, 4 and 6) were upregulated specifically in the virulent vs. mock comparison as was the cytokinin-responsive gene GRXS13. The ARABIDOPSIS RESPONSE REGULATOR genes ARR4, 5,6 , and 7 were upregulated and were among the handful of genes that were found to 
be significantly differentially expressed in the comparison made between the virulent and avirulent treatments. These genes were either downregulated at some point in the avirulent vs. mock comparison (ARR4 and 6) or at least displayed lower mean expression levels at the latter time-points in the avirulent treatment condition compared to the mock treatment. Other $A R R$ genes were downregulated including $A R R 3,11$, and 16 . All of these genes other than $A R R 11$ belong to the type A class of $A R R$ genes, which are known to repress cytokinin signaling (as opposed to the type $B A R R s$, which positively regulate cytokinin signaling. $A R R 11$ is a type $\mathrm{B}$ factor, but it was downregulated in both the avirulent vs. mock and virulent vs. mock comparisons, whereas the type A ARRs represented a module of genes that were significantly upregulated specific to the virulent vs. mock comparison, downregulated specific to the avirulent vs. mock comparison, or both. Figure 3.10 shows expression plots for select cytokinin-related genes.

\section{Genes responding to virulent Agrobacterium treatment}

Several genes that were differentially expressed in the virulent vs. mock comparison, but not in avirulent vs. mock displayed an expression pattern indicating slight, but consistent through time, differences between treatments where the gene was most highly induced or repressed in the virulent condition, but the avirulent condition showed moderate levels in between those of the virulent and mock conditions. Among upregulated genes, are the NAC domain-containing protein NACO01, which was called as differentially expressed at the 12,24 , and 48-hour time-points, and the bHLH transcrip- 
tional regulator ATBS1 Interacting Factor 3 (AIF3). Others examples include the Glutaredoxin family protein GRXS13, which is previously known to be involved in the pathogenesis process of Botrytis cinerea (La Camera et al. 2011), the ubiquitin-protein ligase $A R I 12$ which is known from its role in the cellular response to ultraviolet light (Xie et al. 2015), MPK3/6-targeted VQ motif protein 2 (MVQ2) and AT3G28570, a P-loop containing nucleoside triphosphate hydrolases superfamily protein. Downregulated genes which show a pattern where the highest expression levels are seen in the mock treatment, the lowest levels are seen in the virulent treatment, and where the avirulent treatment shows intermediate levels include early nodulin-like protein 16 (ENODL16), WRKY65, AGL12 and the nodulin MtN21/EamA-like transporter family protein Siliques Are Red 1 (SIAR1).

In addition to the $A R R 4,5,6$, and 7 genes which were upregulated in the virulent vs. avirulent comparison, the LOB domain containing transcription factor ASL9 was upregulated at the 48-hour time-point. Like the ARR genes, ASL9 always showed the lowest expression levels in the avirulent condition and the highest levels in the virulent condition at all time-points. Other genes, though they did not reach the significance threshold for differential expression in the virulent vs. avirulent comparison, showed similar expression trends through time. The $\mathrm{C} 2 \mathrm{H} 2$ transcription factor GLABROUS INFLORESCENCE STEMS3 (GIS3), which is involved in both cytokinin and gibberellin signaling, was one such gene as was the Barwin-related endoglucanase expansin A1 (EXPA1). Arabidopsis ubiquitin-conjugating enzyme 16 (ATUBC16) also displays a pattern where ex- 
pression levels are markedly higher in the virulent treatment, but levels are not necessarily lower in the avirulent treatment than in the mock condition. Two genes coding for enzymes involved in cytokinin catabolism, cytokinin oxidase 3 and 4 (CKX3 and 4) both show a pattern where expression levels are about the same in the avirulent and mock treatments and then are markedly higher in the virulent treatment. Figure 3.11 shows expression plots for select genes that display an apparent, though not necessarily statistically significant, differential response between the virulent and avirulent treatments.

\section{Comparison of RNA-seq results to qRT-PCR data}

Since the two biological replicates used in RNA sequencing were collected and sequenced at two different times of year, resulting in high variance between the samples from each replicate and a large batch effect (which was partially corrected) qRTPCR experiments were performed to assess the reproducibility of differential expression patterns seen in the RNA-seq data for select individual genes. The PAMP response gene FLG22-induced receptor-like kinase 1 (FRK1) was induced by both avirulent and virulent Agrobacterium treatment as early as the 6-hour time-point. qRT-PCR of this gene showed differential expression as beginning later in time at the 12-hour time-point. With the probes used for qRT-PCR, FRK1 levels were below the threshold of detection at all time-points in the mock treatment. The peroxidase 33 gene (PRX33) was another that was strongly upregulated in both Agrobacterium treatments relative to mock. Although qRT-PCR showed the same general trend in expression as RNA-seq, expression of $P R X 33$ peaked at 48 hours in all treatments, which was not the case according to the RNA-seq 
expression data. For another PAMP response gene, respiratory burst oxidase homologue $D(R B O H D), \mathrm{qRT}-\mathrm{PCR}$ again supported the expression pattern seen in the RNA-seq data with the exception that the qRT-PCR data showed a significant difference between the $R B O H D$ expression in the avirulent and virulent Agrobacterium treatment at the 48-hour mark, whereas in the RNA-seq data peak expression levels at 48 hours were approximately equal between the different Agrobacterium treatments. The AP2/ERF transcription factors ERF5 and ERF6, both previously known to be induced in response to PAMPs such as chitin (Moffat et al. 2012), showed higher mean expression levels through time in both Agrobacterium treatments than in the mock treatment. For both genes, expression decreased slightly in all treatments from the 3 to the 12 -hour time-points before increasing in expression at the 24 and 48-hour time-points in both Agrobacterium treatments, while remaining near-constant through time in the mock treatment. RNA-seq data showed no significant difference in expression between the Agrobacterium treatments at the 48-hour time-point for ERF6. Figure 3.12 displays qRT-PCR relative expression plots alongside RNA-seq expression plots for select genes.

\section{Discussion}

In this study, we investigated the transcriptional response of Arabidopsis thaliana seedlings to inoculation with a hypervirulent strain of Agrobacterium tumefaciens (At804) and an otherwise equivalent avirulent strain lacking a Ti plasmid. Though the avirulent strain was compromised in its ability to form a type IV secretion system and to deliver transferred factors (VirD2, VirD5, VirE2, VirE3 and VirF as well as single-stranded 
T-DNA) into host plant cells, it is still able to undergo the attachment process to the outer surface of the plant, as the genes necessary for this are located on the bacterial chromosome, not the Ti plasmid. Thus, even for treatment with the avirulent strain, the plant is able to perceive any extracellular or secreted factors that could function as PAMPs. Based on the overlap seen between the avirulent vs. mock treatment comparison and the virulent strain vs. mock treatment comparison, the majority of differentially expressed genes noted in this study were part of a general response to Agrobacterium stimulus excluding the influence of transferred factors. Many of these are likely altered in response to PAMP perception. It has previously been established that the elongation factor Tu protein of Agrobacterium can not only be perceived by Arabidopsis via the recognition receptor $E F R$, but that this pattern recognition has an inhibitory effect on transformation (Zipfel et al. 2006).

It is likely that Agrobacterium-derived PAMPs other than EF-Tu are also perceived by the plant and thus compound the overall response. Lipopolysaccharide (LPS), a membrane component of all gram-negative bacteria and peptidoglycan (PGN), constituent of the bacterial cell wall, have both been previously shown to induce transcriptional changes indicative of the PAMP response (Gust et al. 2007, Livaja et al. 2008). Perception of PGN is mediated by receptor kinase proteins with LysM domains, particularly LysM domain GPI-anchored protein 1 or $3(L Y M 1 / 3)$ and chitin elicitor receptor kinase 1 (CERK1) (Willmann et al. 2011). This mechanism overlaps with that of chitin perception (a PAMP that allows detection of fungal pathogens). As a result, there is a high amount of overlap in the genes induced in response to either PGN or chitin. This is supported in 
the present study by the fact that the GO biological process term "response to chitin" was significantly enriched among upregulated genes in both the virulent vs. mock and avirulent vs. mock comparisons. LPS is perceived by the lectin S-domain receptor kinase SD1-29 (Ranf et al. 2015) and is a weak inducer of PAMP-triggered immunity (Livaja et al. 2008).

893 genes were upregulated in the virulent vs. mock contrast, but not in the avirulent vs. mock contrast, whereas only 165 genes were upregulated exclusively in the avirulent vs. mock contrast. Most of these genes were called as significantly differentially expressed only in a single contrast (most commonly at the 48-hour time-point). It is predictable that a small fraction of these should represent false positives as a consequence of where the significance threshold was set. Even though very few genes were called as differentially expressed when comparing the virulent and avirulent treatments, the number of additional differentially expressed genes relative to mock treatment observed in response to the virulent strain suggests that some of them must represent a true difference in response even if many did not reach the significance threshold in the comparison between the two Agrobacterium treatments.

When examining expression patterns at the individual gene level, several of the defense-related genes upregulated by treatment with the avirulent Agrobacterium strain had even higher expression levels in the virulent treatment (see Figure 3.11). It is apparent that rather than ameliorating the response to PAMPs such as EF-Tu, LPS, and PGN, delivery of the transferred factors compounds the overall defense response. One 
possibility that could account for this observation is that the Agrobacterium strain carrying the Ti plasmid may contain an unknown PAMP that is detected by the plant and has an additive effect on the defense response. This theoretical PAMP could be any of the transferred Vir proteins mentioned previously, a component of the type IV secretion system (VirB1-11/VirD4), any other protein product encoded by the Ti plasmid, or even the T-DNA itself. There are over 600 receptor-like kinase genes in the Arabidopsis genome and molecular functions have only been described for a few of them (Shiu and Bleecker 2001). Furthermore, there is indirect evidence for the existence of group-specific PAMPs in pathogenic bacteria possessing type III secretion systems (Nürnberger et al. 2004). Thus, it is certainly possible that plants have evolved a mechanism to specifically detect type IV secretion systems or even agrobacteria exclusively.

A few genes showed a stark difference in their expression patterns between the two Agrobacterium treatments. This included a module of type A ARR genes that were among the few genes differentially regulated in the virulent $v s$. avirulent comparison. These genes were strongly upregulated even early in the time-course (beginning at 3 hours post-inoculation for $A R R 5$ and beginning at 6 hours for $A R R 4,6$, and 7) extending through the 24-hour samples. None of them were differentially expressed by 72 hours. These genes are negative regulators of cytokinin signaling. In addition, the cytokinin-metabolizing enzymes $C K X 3, C K X 4$, and $C K X 6$ were highly expressed in the virulent treatment relative to the avirulent and mock treatments. On the other hand, among the downregulated genes were cytokinin signaling components $A H K 2, A H K 5$, and $A H P 1$ as 
well as the positive regulator of cytokinin signaling SOFL1 and two genes involved in cytokinin transport (PUP1 and ABCG14). Downregulation of these genes did not appear to be specific to treatment with the virulent strain as a similar trend was seen in the avirulent condition. However, for the three cytokinin biosynthetic enzymes that were downregulated compared to mock (IPT3, 5, and 7), expression levels were decreased in the avirulent treatment relative to mock, but the effect was clearly compounded in the virulent treatment where expression levels were even lower at each time-point.

Taken together, these observations provide strong evidence that the growth regulating activity of cytokinin is specifically suppressed by the virulent Agrobacterium strain. This is an interesting observation as wild-type (oncogenic) Agrobacterium strains such as C58 contain a bacterial ipt gene within their T-DNA region that is plant-active and leads to the biosynthesis of cytokinin, which promotes their pathogenesis (Britton et al. 2008). In fact, it has been shown that cytokinin secreted by Agrobacterium increases transformation efficiency as it represses a negative transcriptional regulator of transformation susceptibility (Sardesai et al. 2013). Thus, it is puzzling why a hypervirulent Agrobacterium strain would suppress cytokinin biosynthesis and response. It is possible that expression of these genes is tissue-specific even in the 10-day-old seedlings used in this study as auxin can differentially influence the expression of some type A ARR genes depending on whether they are expressed in shoot or root tissue (Paponov et al. 2008). The mechanism by which this might happen is also unclear. Only relatively few factors (the translocated Vir proteins and T-DNA) could potentially be responsible 
for the repression of cytokinin that was observed here. Confirming this observation and elucidating its mechanism could be a subject for future research.

\section{Conclusion}

This work sought to re-analyze the raw RNA-seq data generated for and described in Duan et al. (2018). More contemporary read alignment software was used in addition to a new batch correction technique, new differential expression software, and a different statistical model. This updated method resulted in about $40 \%$ more differentially expressed genes being called than were reported in the original publication. $89 \%$ of the genes called as differentially expressed in the original analysis were conserved in this study and nearly all of the genes that were not retained from the original report were found only in one contrast. Deeper analysis of differentially expressed genes within broad categories such as transcription factors and hormone-related genes helped to generate more specific candidate genes that will serve to inform future work on genetics of the Agrobacterium-plant interaction.

Future research directions based on what was observed in this analysis might involve investigating whether virulent strains of Agrobacterium repress cytokinin signaling at an early time following inoculation in other tissues, transformation contexts, and in other plant species. Whether or not the inhibition of cytokinin signaling has an effect on the efficiency of transformation might also be evaluated by transformation of Arabidopsis seedlings that are multiple mutants for type A ARRs or by the addition of exogenous cytokinin to the co-cultivation buffer. If cytokinin is found to have an influence over the 
transformation process, the mechanism by which the Agrobacterium restrains the activity of endogenous host cytokinins may be investigated by probing for protein-protein interactions between libraries of translocated Vir proteins and host genes regulating early cytokinin signaling. Such an exploration would be simplified by the fact that there is a relatively small pool of candidate molecules on the pathogen side. Identification of additional genes that facilitate the successful delivery of T-DNA from agrobacteria into plant cells will not only enhance our understanding of the Agrobacterium pathogenesis process, but will contribute to the advancement of genetic engineering technologies used to improve plant traits.

\section{Materials and Methods}

Plant and bacterial genotypes used

Seedlings of the Col-0 ecotype of Arabidopsis thaliana were used in this study for Agrobacterium inoculation. After being sterilized with a $10 \%$ bleach solution and a $70 \%$ ethanol wash followed by five rinses with deionized distilled water, wild-type Col-0 seeds were aseptically plated on MS media and refrigerated at $4^{\circ} \mathrm{C}$ for 3 days and then incubated at $25^{\circ} \mathrm{C}$ under a $12 / 12$ photoperiod for 10 days until the germinated seedlings were used for transient transformation experiments. Both Agrobacterium tumefaciens strains used in this study were derived from disarmed strain EHA105. Strain A136 has been cured of its Ti plasmid and is thus deficient in the ability to transmit Vir proteins and T-DNA to plant cells as well as in the assembly of a type IV secretion system. A136 was referred to as the "avirulent" strain in this study. Strain At804 contains the super- 
virulent Ti plasmid pTiBo542 as well as the binary plasmid pBISN1 (Ni et al. 1995), which carries a uidA (GUS)-intron sequence within its T-DNA region, rendering this marker gene expressible only in plant cells. At804 was, in this study, referred to as the "virulent" strain.

Transient seedling transformation and sample collection

The AGROBEST assay, as described by Wu et al. (2014), was used in this study to perform transient transformation of whole Arabidopsis seedlings grown as described above prior to collection, at various time-points following inoculation, for RNA-sequencing. Briefly, pools of about 25 10-day-old seedlings were treated with either of the two strains of Agrobacterium suspended in inoculation buffer at a density of $108 \mathrm{CFU} / \mathrm{mL}$ or received mock treatment with inoculation buffer only. Petri plates containing seedlings co-cultivated with Agrobacterium suspensions were incubated at $25^{\circ} \mathrm{C}$ under a $12 / 12$ photoperiod with gentle shaking (approximately $70 \mathrm{rpm}$ ). Seedling samples were collected at $0,3,6,12,24$, and 48 hours following inoculation. Seedlings were blotted dry with Kim-wipes, were placed into $1.7 \mathrm{~mL}$ microcentrifuge tube, and were flash-frozen in liquid nitrogen until they could be processed.

Total RNA was extracted from frozen seedling tissue using the TRIzol reagent following the accompanying protocol (Invitrogen, http://www.invitrogen.com). RNA integrity was assessed by agarose gel electrophoresis and was quantified and assessed for DNA or protein contamination using a NanoDrop. Approximately $5 \mu \mathrm{g}$ of total RNA from 
each sample was submitted for mRNA processing, cDNA library construction and sequencing to the University of Missouri DNA Core Facility. Read libraries were produced using the Illumina TruSeq (v3) RNA sample preparation protocol and sequenced using the Illumina HiSeq 2500 platform. Raw read data was delivered in FASTQ format following quality control analysis. Two biological replicates were employed for RNA sequencing. Samples for these two experimental replications were collected, processed, and sequenced several months apart. Transient transformation, sample collection, and total RNA extraction was performed by Dr. Kaixuan Duan.

\section{Data processing and gene expression quantification}

Raw RNA-seq reads were quality trimmed to remove adapter sequences and low-quality regions using Trimmomatic version-0.35 (Bolger et al. 2014) with the "Sliding window" method and discarding reads trimmed shorter than 40 bases in length. The sets were then "cleaned" of Arabidopsis thaliana mitochondrial DNA and ribosomal DNA sequences, which were acquired from the NCBI genome database and SILVA (Quast et al. 2012), respectively, by reverse aligning the trimmed reads to a custom database containing these sequences using Bowtie2 (Langmead et al. 2019) with the --un option enabled. Trimmed and cleaned reads were then mapped to the A. thaliana Araport11 reference genome (Cheng et al. 2017), which was downloaded from The Arabidopsis Information Resource (TAIR) FTP site, using HISAT2 (Kim et al. 2015) with the --dta tag option enabled. Per-gene read counts were generated for each sample with featureCounts (Liao et al. 2014) using the GFF3 file accompanying the Araport 11 assembly to provide 
gene annotations along with the SAM file generated from the read alignment for each sample. Raw counts for each sample were combined into a single read count data set in preparation for differential expression analysis.

\section{Analysis of differential gene expression}

Differential expression analysis was performed using DESeq2 (Love et al. 2014), which functions by fitting each gene to a negative binomial generalized linear model. Log-transformed expression values for each of the samples were visualized by PCA plotting, which showed an apparent separation of groups by biological replicate. Thus, the batch correction software ComBat-seq (Zhang et al. 2020) was employed to help compensate for the batch effect (see Figure 3.1). The corrected read count data set was then analyzed by DESeq2. Only genes with total read count values greater than or equal to 10 across all samples were retained for further analysis. Samples were grouped by time, treatment, and replicate and the study design matrix used treatment as the primary factor. Contrasts were performed between each pairing of samples from the three experimental treatments at equivalent time-points. Differences were evaluated using the Wald test. The Benjamini-Hochberg procedure was used to obtain false discovery rate (FDR) values for each gene-wise test. Genes with at least a 2-fold expression change and an FDR value below 0.05 for a given contrast were considered differentially expressed for the purposes of this study.

Scatterplots, clustering, and gene set enrichment analysis 
Scatterplots were generated by log2-transforming the counts per million reads (CPM) values for each gene after filtering for those with very low read counts. The Scatterplot3d R package (Ligges and Mächler 2002) was used to plot expression levels from all three treatments against each other. Venn diagrams were generated, using the online tool BioVenn, by pasting sets of differentially expressed genes in each comparison into the webpage (Hulsen et al. 2008). Co-expression clusters were generated by inputting log2-transformed CPM values for each differentially expressed gene within each sample into the clust software package (Abu-Jamous and Kelly 2018) and setting the tightness parameter (-t) to 2.0 and the normalization parameter (-n) to 0 . The plot visually representing mean expression values for each cluster was generated using the $R$ package gplots (Warnes et al. 2009). Enrichment of various gene set terms was analyzed using the Database for Annotation, Visualization and Integrated Discovery (DAVID) online tool (https://david.ncifcrf.gov/home.jsp) (Sherman and Lempicki 2009). Lists of differentially expressed genes that were either upregulated or downregulated in comparisons between two conditions at each time-point were tested against the full Arabidopsis genome background using Fisher's exact test according to the number of genes annotated for each category. The Bonferroni method was used to make the FDR adjustment. Gene functional sets with an FDR value less than 0.05 were considered significantly enriched. The list of enriched functional terms was reduced using manual inspection to eliminate redundant and irrelevant terms.

Transcription factor analysis 
Annotated lists of transcription factor genes in the $A$. thaliana genome were obtained from the Plant Transcription Factor Database version 5.0 (PlantTFDB; http://planttfdb.gao-lab.org/) (Jin et al. 2016). Custom scripts were used to count the number of members of each transcription factor family falling into sets of upregulated or downregulated genes for each contrast.

Evaluating expression trends with qRT-PCR

Total RNA was extracted from samples of $A$. thaliana seedlings that received the same treatments as described above in preparation for RNA-seq. This was then partially converted to cDNA by first-strand synthesis using reverse transcription with oligo dT primers. qRT-PCR was performed using 96-well plates with a Bio-Rad CFX96 instrument. iQ SYBR green supermix (Bio-Rad) was used for fluorescent detection of amplicons. $10 \mu \mathrm{L}$ reaction volumes were run for 40 cycles using the $A$. thaliana $U B Q 10$ gene as an internal standard. Three biological replicates were used and replicate reactions using each sample template were run in three wells for each. Data analysis was performed using the BioRad CFX Manager 2.0 software. The $\Delta \Delta \mathrm{Ct}$ (comparative cycle threshold) method was used the calculate the relative fold-change in expression of target genes. Transient transformation, RNA extraction, cDNA conversion, and qRT-PCR experiments were performed by Dr. Kaixuan Duan.

\section{Reference List}

Abu-Jamous B, Kelly S. 2018. Clust: automatic extraction of optimal co-expressed gene clusters from gene expression data. Genome biology 19: 1-11. 
Aguilar J, Cameron TA, Zupan J, Zambryski P. 2011. Membrane and core periplasmic Agrobacterium tumefaciens virulence type IV secretion system components localize to multiple sites around the bacterial perimeter during lateral attachment to plant cells. MBio 2.

Bauer Z, Gómez-Gómez L, Boller T, Felix G. 2001. Sensitivity of different ecotypes and mutants of Arabidopsis thaliana toward the bacterial elicitor flagellin correlates with the presence of receptor-binding sites. Journal of Biological Chemistry 276: 45669-45676.

Bevan MW, Flavell RB, Chilton M-D. 1983. A chimaeric antibiotic resistance gene as a selectable marker for plant cell transformation. Nature 304: 184-187.

Binns D, Dimmer E, Huntley R, Barrell D, O'donovan C, Apweiler R. 2009. QuickGO: a web-based tool for Gene Ontology searching. Bioinformatics 25: 3045-3046.

Bolger AM, Lohse M, Usadel B. 2014. Trimmomatic: a flexible trimmer for Illumina sequence data. Bioinformatics 30: 2114-2120.

Britton MT, Escobar MA, Dandekar AM. 2008. The oncogenes of Agrobacterium tumefaciens and Agrobacterium rhizogenes. Pages 523-563. Agrobacterium: from biology to biotechnology, Springer.

Cheng CY, Krishnakumar V, Chan AP, Thibaud-Nissen F, Schobel S, Town CD. 2017. Araport11: a complete reannotation of the Arabidopsis thaliana reference genome. The Plant Journal 89: 789-804.

Chinchilla D, Bauer Z, Regenass M, Boller T, Felix G. 2006. The Arabidopsis receptor kinase $F L S 2$ binds flg22 and determines the specificity of flagellin perception. The Plant Cell 18: 465-476.

De Cleene M, De Ley J. 1976. The host range of crown gall. The botanical review 42: 389466.

Douglas C, Halperin W, Nester E. 1982. Agrobacterium tumefaciens mutants affected in attachment to plant cells. Journal of bacteriology 152: 1265-1275.

Duan K, Willig CJ, De Tar JR, Spollen WG, Zhang ZJ. 2018. Transcriptomic analysis of Arabidopsis seedlings in response to an Agrobacterium-mediated transformation process. Molecular plant-microbe interactions 31: 445-459. 
Gao C, Nielsen KK. 2013. Comparison between Agrobacterium-mediated and direct gene transfer using the gene gun. Pages 3-16. Biolistic DNA Delivery, Springer.

Gassmann W, Bhattacharjee S. 2012. Effector-triggered immunity signaling: from genefor-gene pathways to protein-protein interaction networks. Molecular PlantMicrobe Interactions 25: 862-868.

Gelvin SB. 2006. Agrobacterium virulence gene induction. Agrobacterium protocols: 7785.

Gelvin SB. 2017. Integration of Agrobacterium T-DNA into the plant genome. Annual review of genetics 51: 195-217.

Gust AA, Biswas R, Lenz HD, Rauhut T, Ranf S, Kemmerling B, Götz F, Glawischnig E, Lee J, Felix G. 2007. Bacteria-derived peptidoglycans constitute pathogen-associated molecular patterns triggering innate immunity in Arabidopsis. Journal of Biological Chemistry 282: 32338-32348.

Herrera-Estrella A, Van Montagu M, Wang K. 1990. A bacterial peptide acting as a plant nuclear targeting signal: the amino-terminal portion of Agrobacterium VirD2 protein directs a beta-galactosidase fusion protein into tobacco nuclei. Proceedings of the National Academy of Sciences 87: 9534-9537.

Hoekema A, Hirsch PR, Hooykaas PJ, Schilperoort RA. 1983. A binary plant vector strategy based on separation of vir-and T-region of the Agrobacterium tumefaciens Ti-plasmid. Nature 303: 179-180.

Hulsen T, de Vlieg J, Alkema W. 2008. BioVenn-a web application for the comparison and visualization of biological lists using area-proportional Venn diagrams. BMC genomics 9: 1-6.

Hwang EE, Wang MB, Bravo JE, Banta LM. 2015. Unmasking host and microbial strategies in the Agrobacterium-plant defense tango. Frontiers in plant science 6: 200.

Jin J, Tian F, Yang D-C, Meng Y-Q, Kong L, Luo J, Gao G. 2016. PlantTFDB 4.0: toward a central hub for transcription factors and regulatory interactions in plants. Nucleic acids research: gkw982.

Kim D, Langmead B, Salzberg SL. 2015. HISAT: a fast spliced aligner with low memory requirements. Nature methods 12: 357-360. 
La Camera S, L'Haridon F, Astier J, Zander M, Abou-Mansour E, Page G, Thurow C, Wendehenne D, Gatz C, Métraux JP. 2011. The glutaredoxin ATGRXS13 is required to facilitate Botrytis cinerea infection of Arabidopsis thaliana plants. The Plant Journal 68: 507-519.

Lacroix B, Citovsky V. 2019. Pathways of DNA transfer to plants from Agrobacterium tumefaciens and related bacterial species. Annual review of phytopathology 57: 231-251.

Langmead B, Wilks C, Antonescu V, Charles R. 2019. Scaling read aligners to hundreds of threads on general-purpose processors. Bioinformatics 35: 421-432.

Liao Y, Smyth GK, Shi W. 2014. featureCounts: an efficient general purpose program for assigning sequence reads to genomic features. Bioinformatics 30: 923-930.

Ligges U, Mächler M. 2002. Scatterplot3d-an R package for visualizing multivariate data. Technical Report. Report no.

Livaja M, Zeidler D, Von Rad U, Durner J. 2008. Transcriptional responses of Arabidopsis thaliana to the bacteria-derived PAMPs harpin and lipopolysaccharide. Immunobiology 213: 161-171.

Love MI, Huber W, Anders S. 2014. Moderated estimation of fold change and dispersion for RNA-seq data with DESeq2. Genome biology 15: 1-21.

Mello-Farias PCd, Chaves ALS. 2008. Advances in Agrobacterium-mediated plant transformation with enphasys on soybean. Scientia Agricola 65: 95-106.

Moffat CS, Ingle RA, Wathugala DL, Saunders NJ, Knight H, Knight MR. 2012. ERF5 and ERF6 play redundant roles as positive regulators of JA/Et-mediated defense against Botrytis cinerea in Arabidopsis. PloS one 7: e35995.

Ni M, Cui D, Einstein J, Narasimhulu S, Vergara CE, Gelvin SB. 1995. Strength and tissue specificity of chimeric promoters derived from the octopine and mannopine synthase genes. The Plant Journal 7: 661-676.

Nürnberger T, Brunner F, Kemmerling B, Piater L. 2004. Innate immunity in plants and animals: striking similarities and obvious differences. Immunological reviews 198: 249-266. 
Paponov IA, Paponov M, Teale W, Menges M, Chakrabortee S, Murray JA, Palme K. 2008. Comprehensive transcriptome analysis of auxin responses in Arabidopsis. Molecular Plant 1: 321-337.

Quast C, Pruesse E, Yilmaz P, Gerken J, Schweer T, Yarza P, Peplies J, Glöckner FO. 2012. The SILVA ribosomal RNA gene database project: improved data processing and web-based tools. Nucleic acids research 41: D590-D596.

Ranf S, Gisch N, Schäffer M, Illig T, Westphal L, Knirel YA, Sánchez-Carballo PM, Zähringer U, Hückelhoven R, Lee J. 2015. A lectin S-domain receptor kinase mediates lipopolysaccharide sensing in Arabidopsis thaliana. Nature immunology 16: 426-433.

Sardesai N, Lee L-Y, Chen H, Yi H, Olbricht GR, Stirnberg A, Jeffries J, Xiong K, Doerge RW, Gelvin SB. 2013. Cytokinins secreted by Agrobacterium promote transformation by repressing a plant Myb transcription factor. Science Signaling 6: ra100-ra100.

Sherman BT, Lempicki RA. 2009. Systematic and integrative analysis of large gene lists using DAVID bioinformatics resources. Nature protocols 4: 44.

Shiu S-H, Bleecker AB. 2001. Receptor-like kinases from Arabidopsis form a monophyletic gene family related to animal receptor kinases. Proceedings of the National Academy of Sciences 98: 10763-10768.

Sinkar VP, White FF, Gordon MP. 1987. Molecular biology of Ri-plasmid-a review. Journal of Biosciences 11: 47-57.

Torisky R, Kovacs L, Avdiushko S, Newman J, Hunt A, Collins G. 1997. Development of a binary vector system for plant transformation based on the supervirulent Agrobacterium tumefaciens strain Chry5. Plant Cell Reports 17: 102-108.

Tzfira T, Citovsky V. 2001. Comparison between nuclear localization of nopaline-and octopine-specific Agrobacterium VirE2 proteins in plant, yeast and mammalian cells. Molecular plant pathology 2: 171-176.

Tzfira T, Citovsky V. 2006. Agrobacterium-mediated genetic transformation of plants: biology and biotechnology. Current opinion in biotechnology 17: 147-154.

Veena, Jiang H, Doerge R, Gelvin SB. 2003. Transfer of T-DNA and Vir proteins to plant cells by Agrobacterium tumefaciens induces expression of host genes involved in 
mediating transformation and suppresses host defense gene expression. The Plant Journal 35: 219-236.

Warnes GR, Bolker B, Bonebakker L, Gentleman R, Huber W, Liaw A, Lumley T, Maechler $M$, Magnusson A, Moeller S. 2009. gplots: Various R programming tools for plotting data. R package version 2: 1.

Willmann R, Lajunen HM, Erbs G, Newman M-A, Kolb D, Tsuda K, Katagiri F, Fliegmann J, Bono J-J, Cullimore JV. 2011. Arabidopsis Iysin-motif proteins LYM1 LYM3 CERK1 mediate bacterial peptidoglycan sensing and immunity to bacterial infection. Proceedings of the National Academy of Sciences 108: 19824-19829.

Wu H-Y, Liu K-H, Wang Y-C, Wu J-F, Chiu W-L, Chen C-Y, Wu S-H, Sheen J, Lai E-M. 2014. AGROBEST: an efficient Agrobacterium-mediated transient expression method for versatile gene function analyses in Arabidopsis seedlings. Plant methods 10: 1-16.

Xie L, Lang-Mladek C, Richter J, Nigam N, Hauser M-T. 2015. UV-B induction of the E3 ligase ARIADNE12 depends on CONSTITUTIVELY PHOTOMORPHOGENIC 1. Plant Physiology and Biochemistry 93: 18-28.

Xiong W, Ye T, Yao X, Liu X, Ma S, Chen X, Chen ML, Feng YQ, Wu Y. 2018. The dioxygenase GIM2 functions in seed germination by altering gibberellin production in Arabidopsis. Journal of integrative plant biology 60: 276-291.

Zhang J, Zhou J-M. 2010. Plant immunity triggered by microbial molecular signatures. Molecular plant 3: 783-793.

Zhang Y, Parmigiani G, Johnson WE. 2020. ComBat-Seq: batch effect adjustment for RNA-Seq count data. NAR genomics and bioinformatics 2: Iqaa078.

Zipfel C. 2009. Early molecular events in PAMP-triggered immunity. Current opinion in plant biology 12: 414-420.

Zipfel C, Kunze G, Chinchilla D, Caniard A, Jones JD, Boller T, Felix G. 2006. Perception of the bacterial PAMP EF-Tu by the receptor EFR restricts Agrobacterium-mediated transformation. Cell 125: 749-760. 
A)

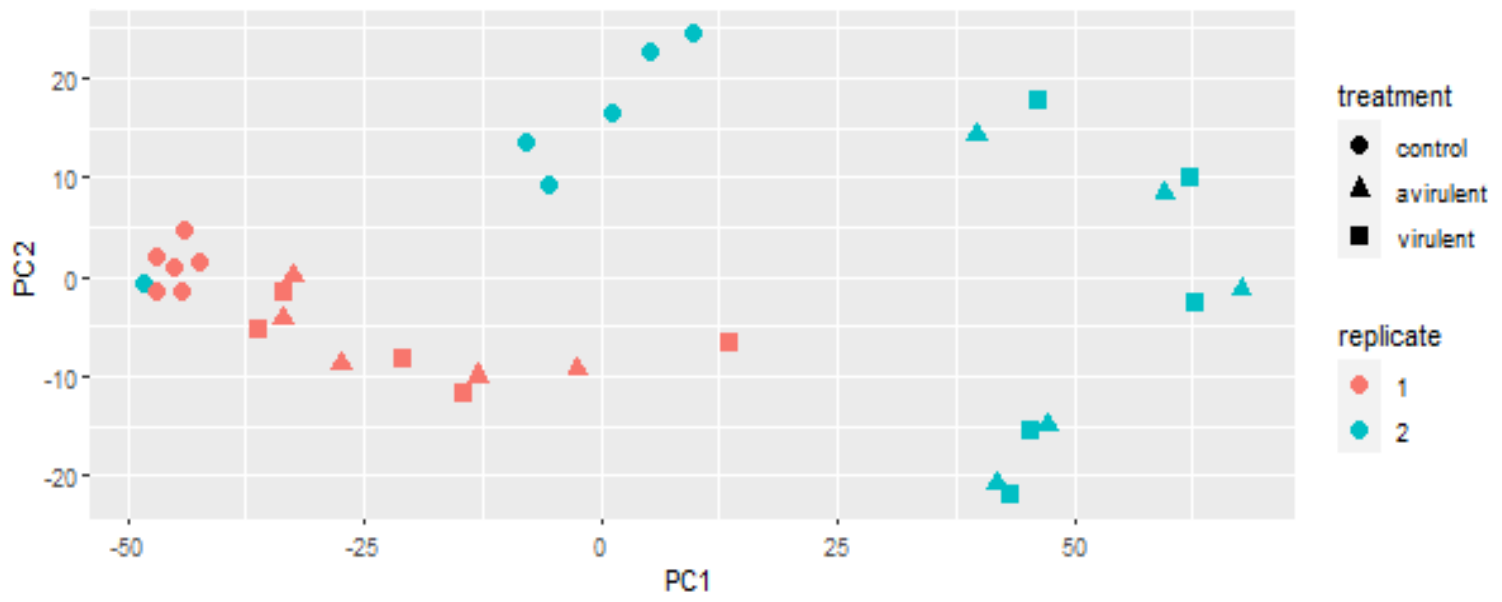

B)

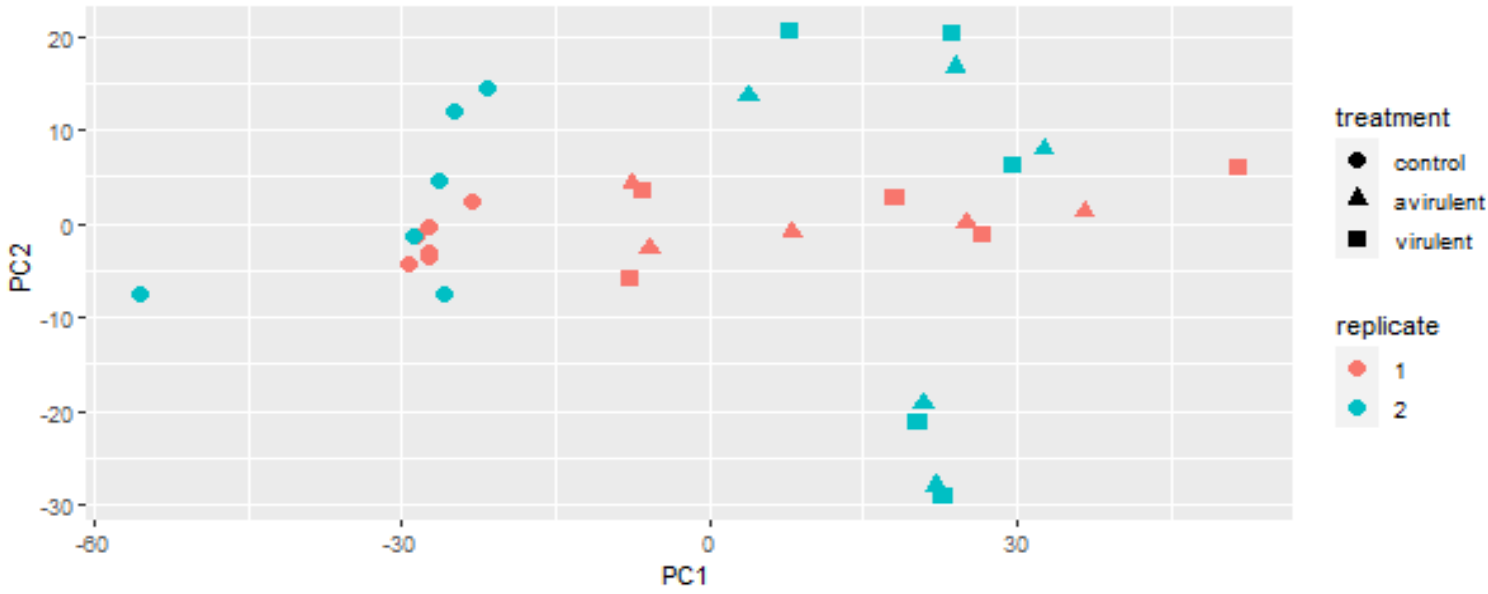

Figure 3.1: PCA plot showing sample distribution. A) A principal components plot of

RNA-seq samples prior to analysis showed a clear separation between different biological replicates. B) Following batch correction, samples were more uniformly distributed. 

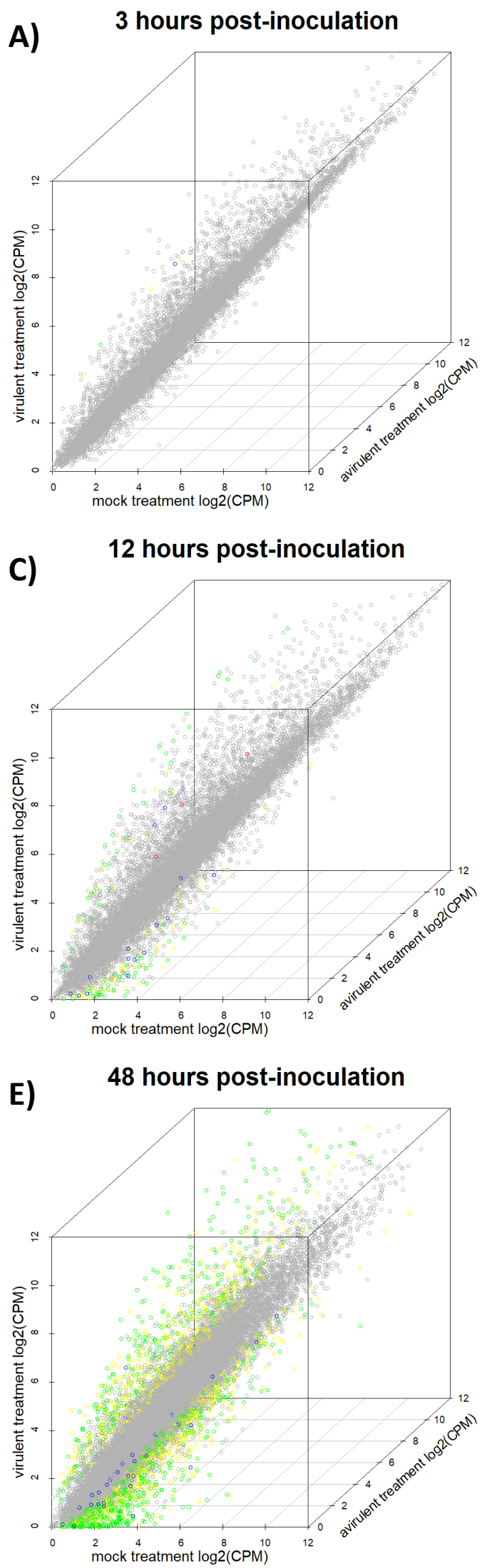
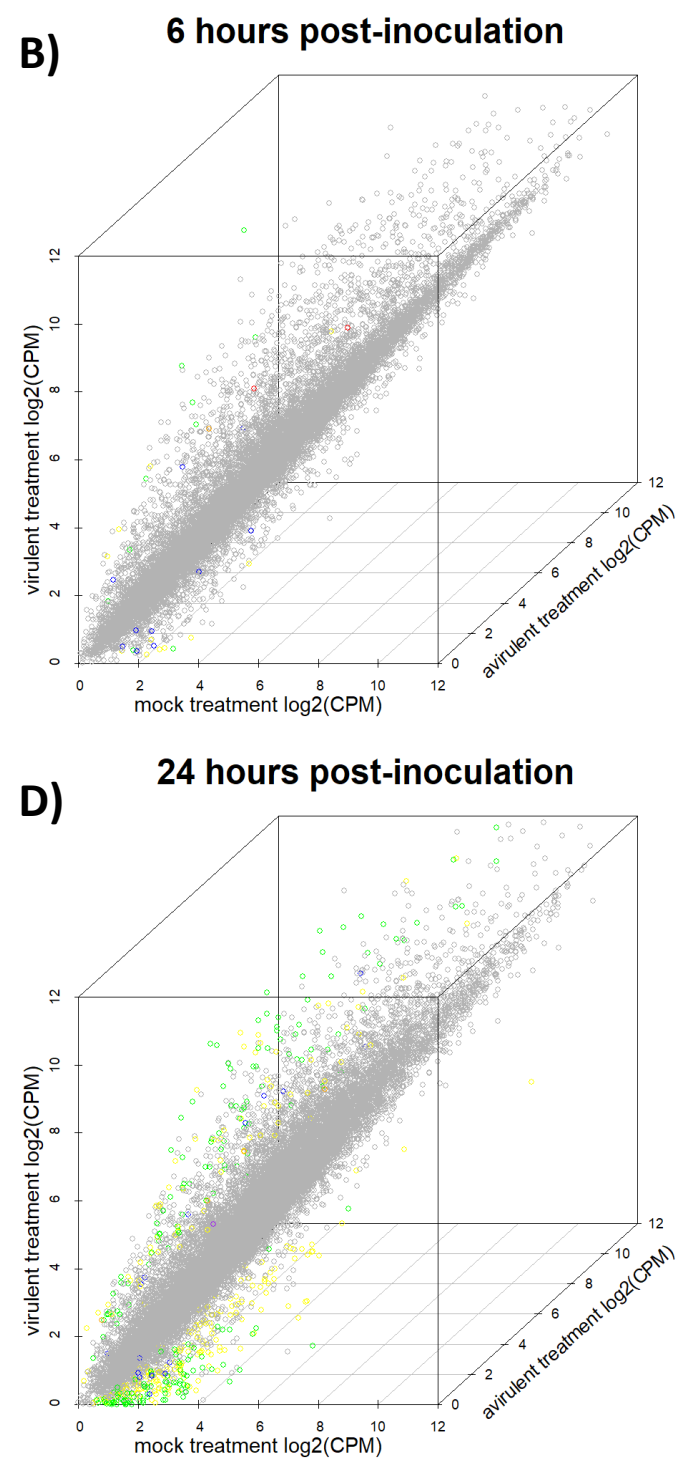

$\square$ not DE in any comparisons

- DE in mock vs. avirulent comparison only

$\square \mathrm{DE}$ in mock vs. virulent comparison only

DE in avirulent vs. virulent comparison only

$\square$ DE in both mock vs, avirulent and mock vs, virulent comparisons

$\square D E$ in both mock vs. virulent and avirulent vs. virulent comparisons

$\square$ DE in both mock vs. avirulent and avirulent vs. virulent comparisons

aE in all 3 comparisons 
Figure 3.2: Scatterplots displaying relative expression of all genes in each treatment at each time-point. 3-dimensional scatterplots showing relative expression, in $\log _{2}$-transformed counts per million of analyzed genes in each of the three treatments at each time-point: A) 3 hours, B) 6 hours, C) 12 hours, D) 24 hours, and E) 48 hours post-inoculation. Points are colored according to what comparisons, if any, they were significantly differentially expressed in at the individual time-point. 
A) All upregulated genes

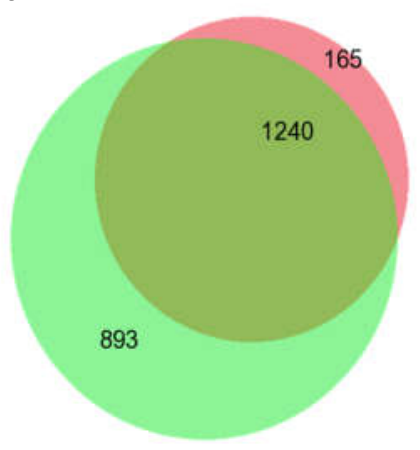

D $12 \mathrm{~h}$ upregulated genes
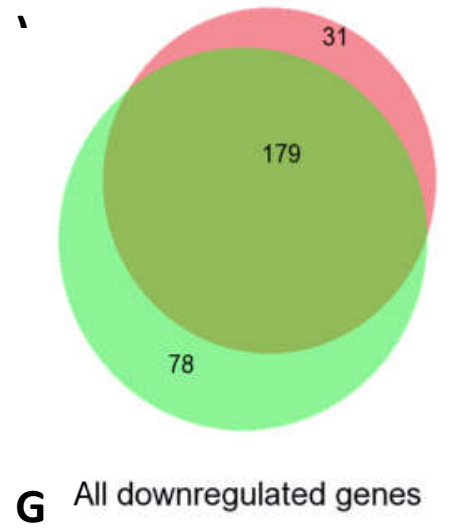

I

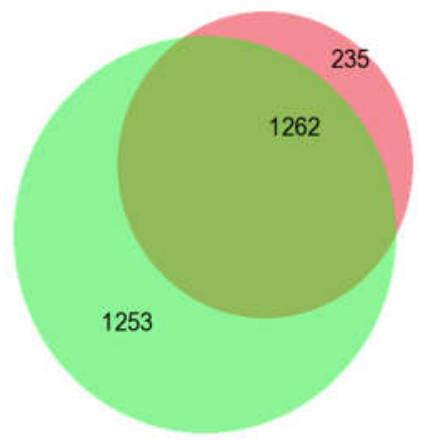

J) $24 \mathrm{~h}$ downregulated genes

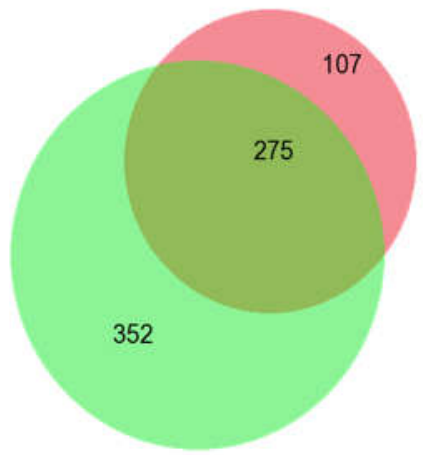

B) 3h upregulated genes

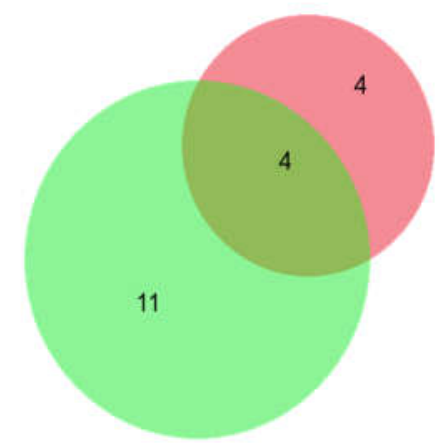

E) $24 \mathrm{~h}$ upregulated genes

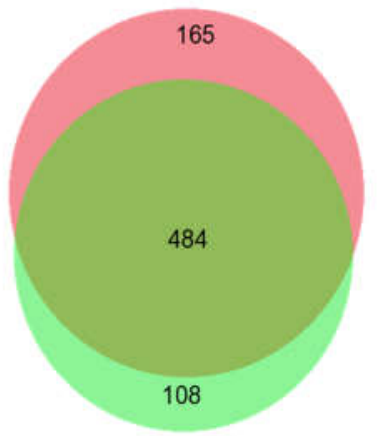

H 6 h downregulated genes

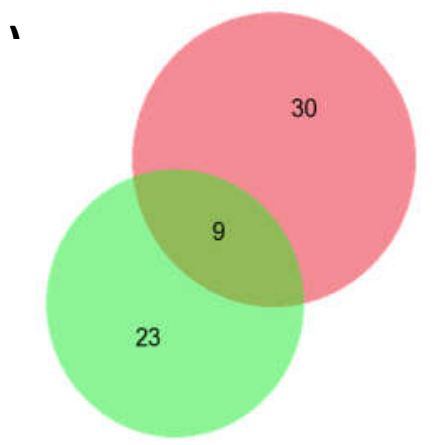

K) 48h downregulated genes

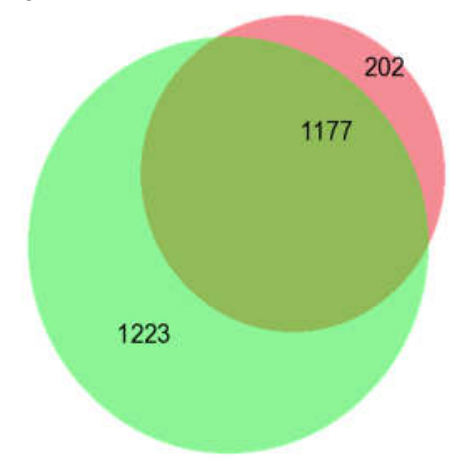

C) $6 \mathrm{~h}$ upregulated genes

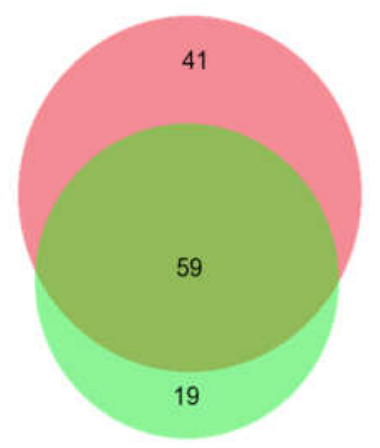

F) $48 \mathrm{~h}$ upregulated genes

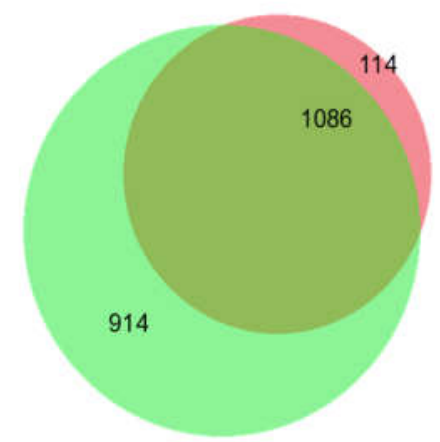

I) $12 \mathrm{~h}$ downregulated genes

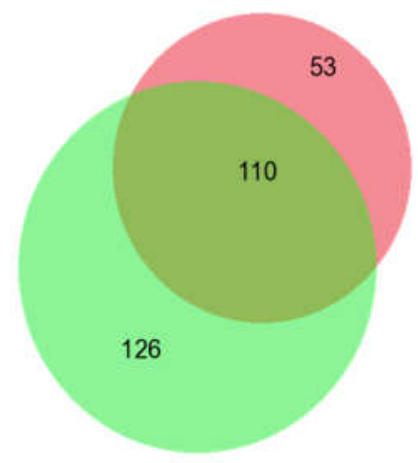

Avirulent vs. mock comparison

Virulent vs. mock comparison

Overlap between comparisons 
Figure 3.3: Venn diagrams showing the differentially expressed gene sets of upregulated, A-F), or downregulated, G-K), genes which are unique to the avirulent vs. mock comparison, unique to the virulent vs. mock comparison, or shared by both. Panel A) displays sets of all genes that were upregulated in at least one contrast for each comparison, while B-F) show upregulated genes at each individual time-point. Panel G) shows sets of all genes that were downregulated in at least one contrast in each comparison, while $\mathbf{H}-\mathbf{K}$ ) show upregulated genes at each individual time-point. Note that only one gene was downregulated at 3 hours in the virulent vs. mock comparison and none were downregulated at 3 hours in the avirulent vs. mock comparison. Thus, a figure was not constructed for these sets. 

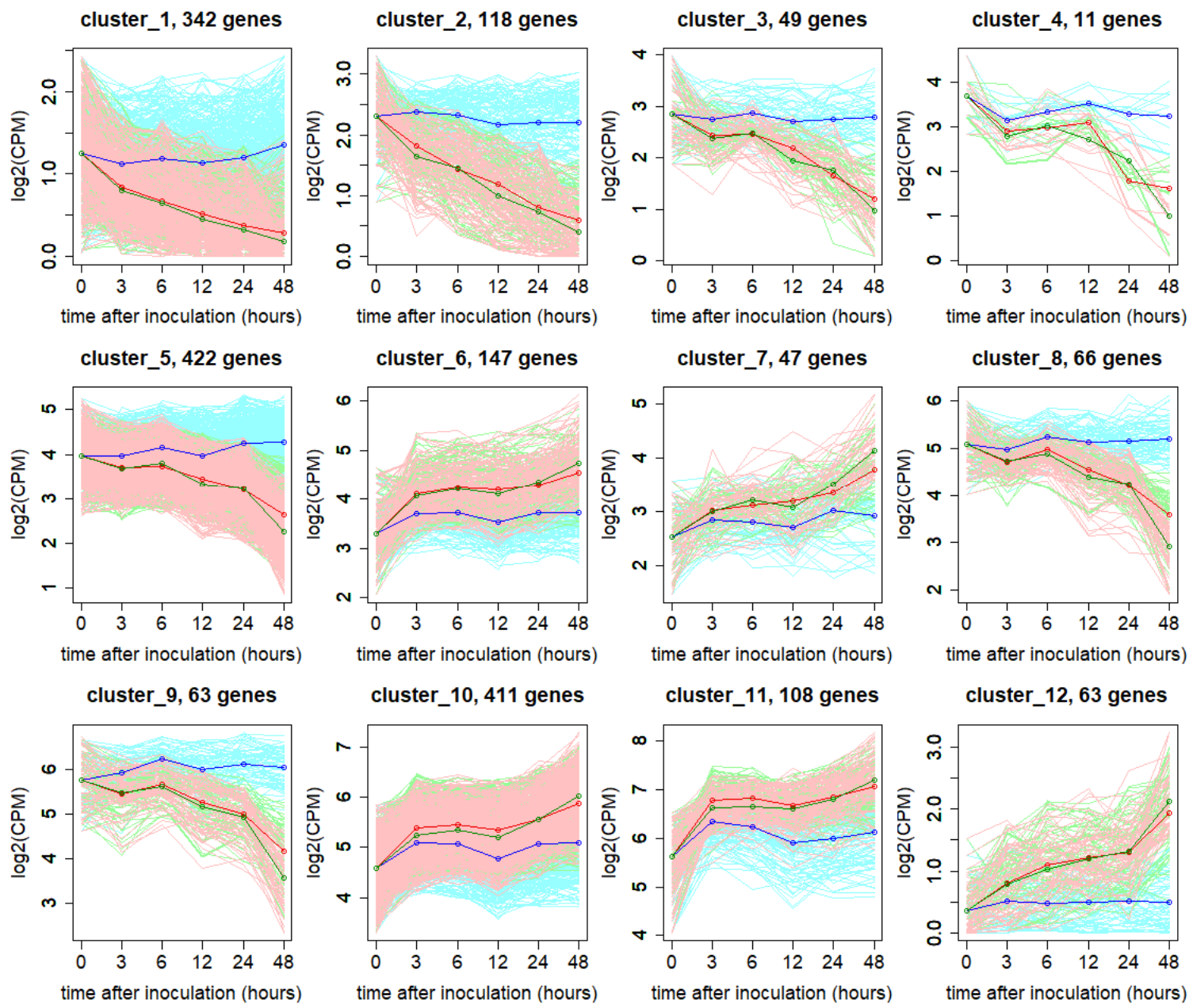

Figure 3.4: Co-expression clustering of differentially expressed genes. For each panel, the solid lines show the mean expression trends through time for all members of the cluster, while the lighter-colored trace lines show the expression trends for individual genes within the cluster. Expression in the mock treatment is in blue, expression in the avirulent treatment is in red, and expression in the virulent treatment is in green. 
A)

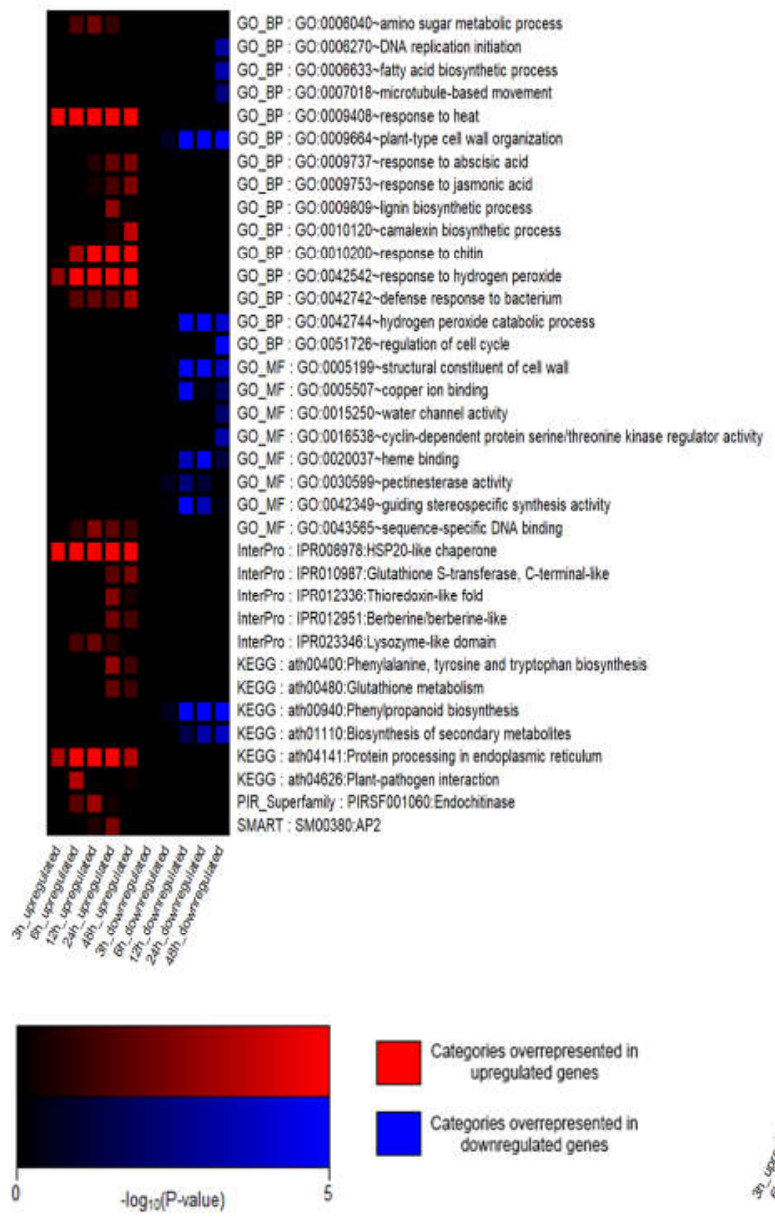

B)

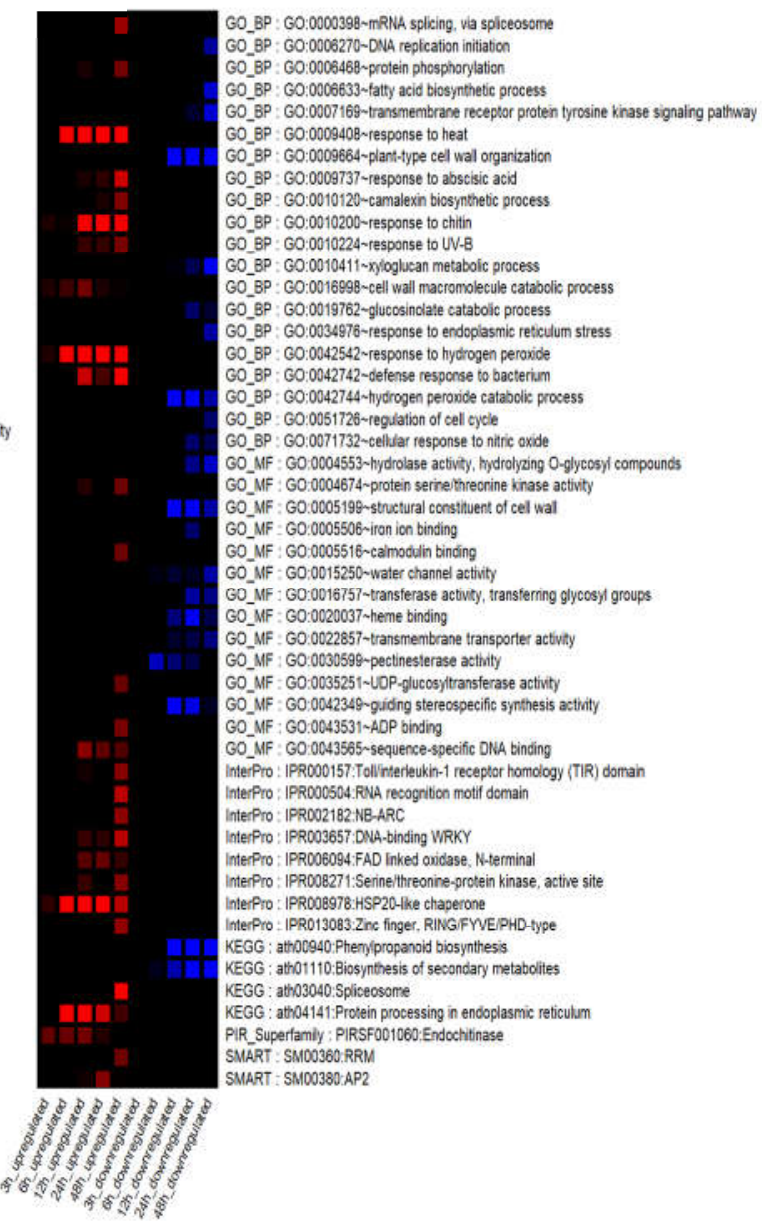

Figure 3.5: Selected overrepresented gene sets among upregulated and downregu-

lated genes at each cross-condition contrast. Degree of enrichment for each category is represented by taking the $-\log _{10}$ transformation of the FDR resulting from tests between counts of genes falling within each gene set category from individual lists of upregulated (increasing saturation of red color) or downregulated (increasing saturation of blue color) genes in each contrast and gene counts from the whole $A$. thaliana genome background. Panel A) shows enriched sets in the avirulent vs. mock comparison, while panel B) displays enriched sets in the virulent vs. mock comparison. 


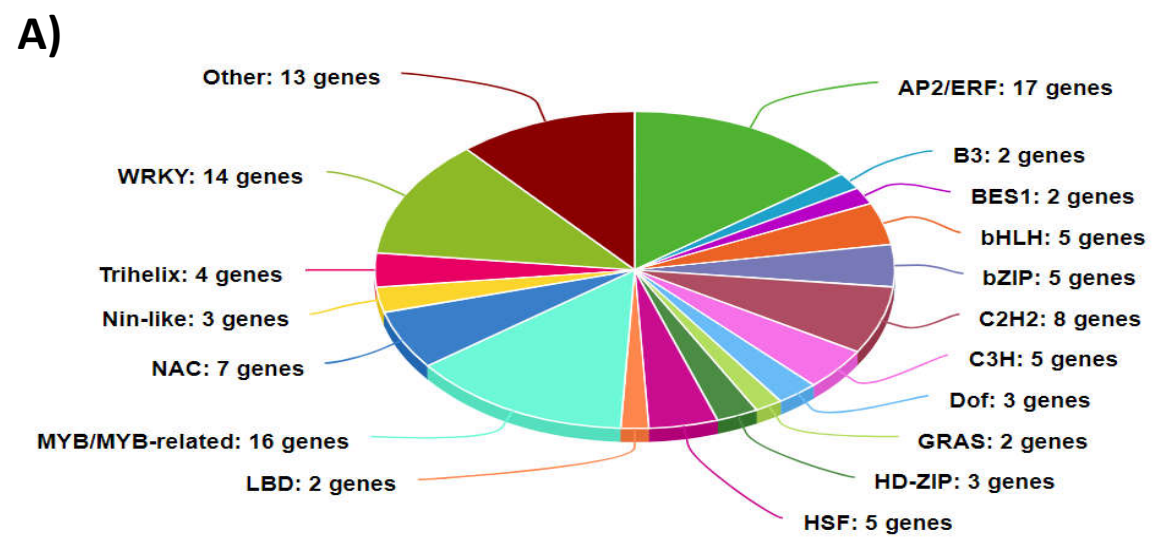

B)

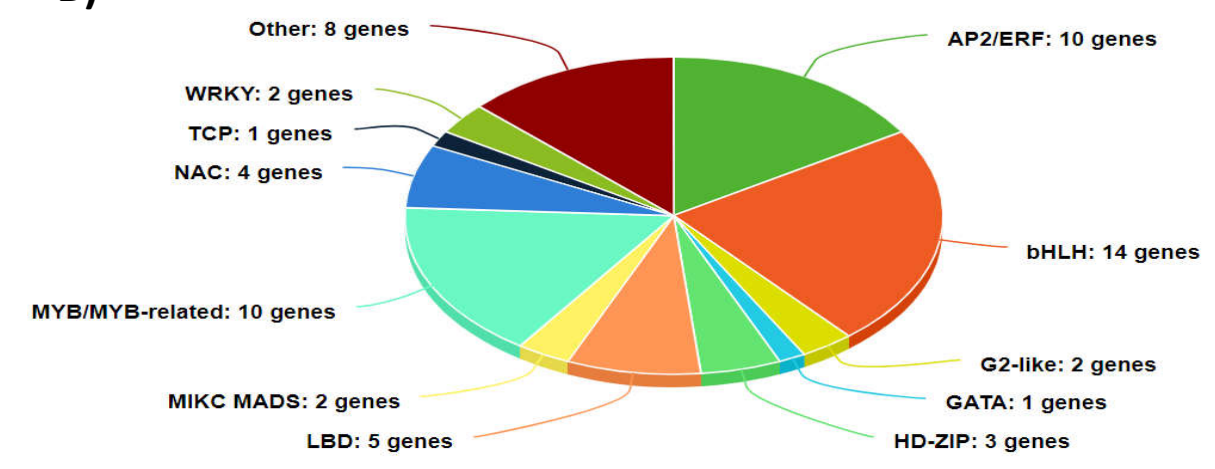

C)

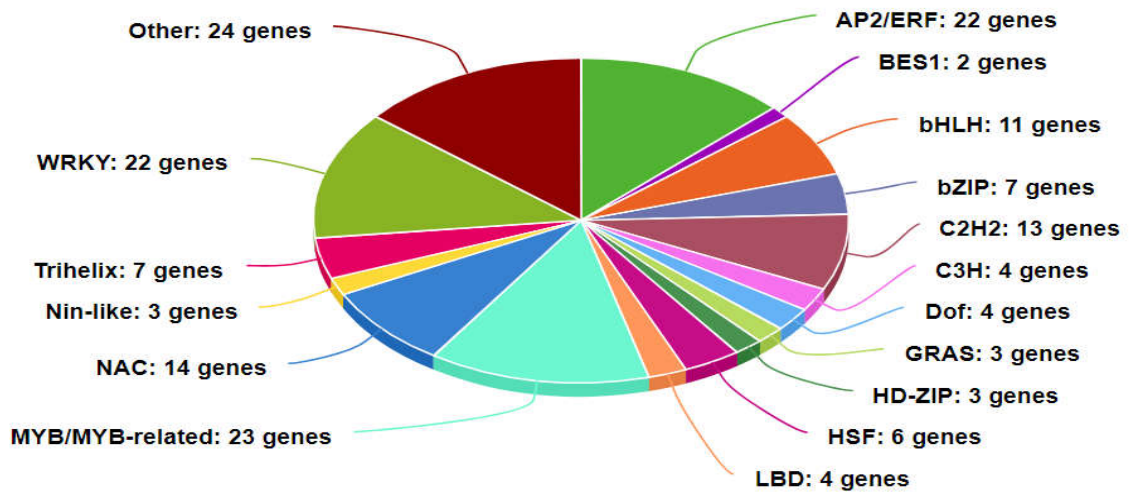

D)

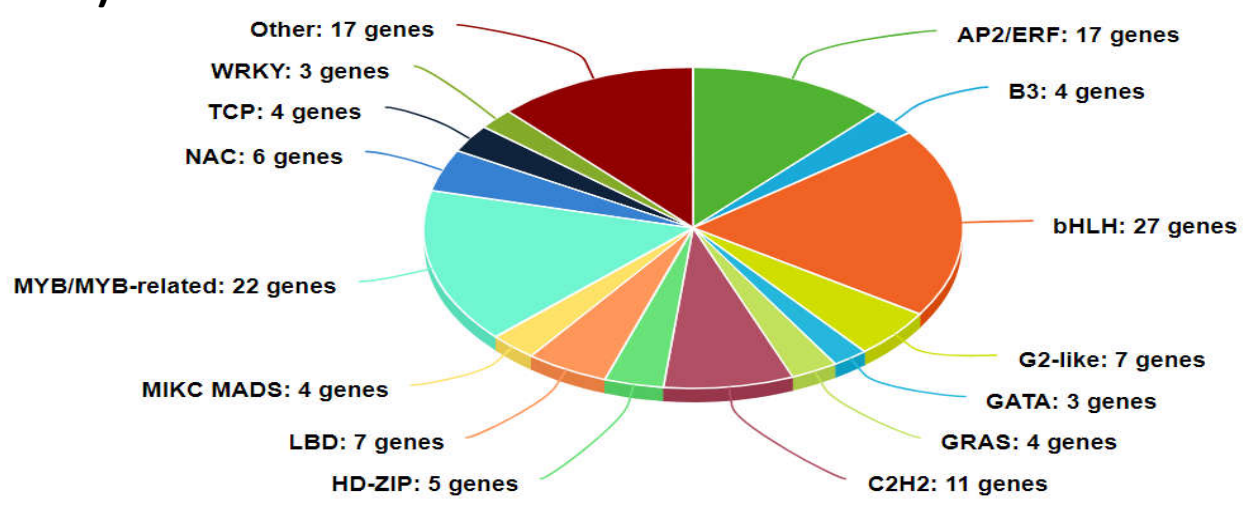


Figure 3.6: Pie charts showing differentially expressed transcription factor genes within sets of those upregulated or downregulated in each comparison. Panel A) shows TF genes that were upregulated in the avirulent vs. mock comparison, B) shows TFs that were downregulated in the avirulent vs. mock comparison, C) shows TFs that were upregulated in the virulent vs. mock comparison, and D) shows TFs that were downregulated in the virulent vs. mock comparison. 
A)
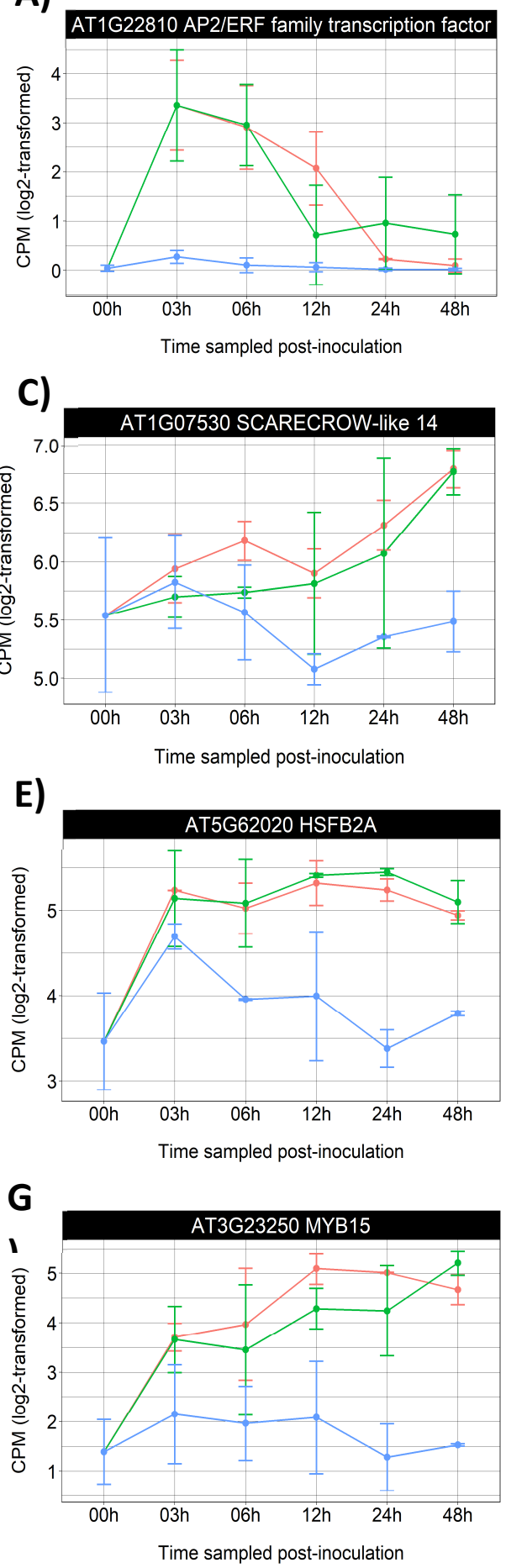

B)
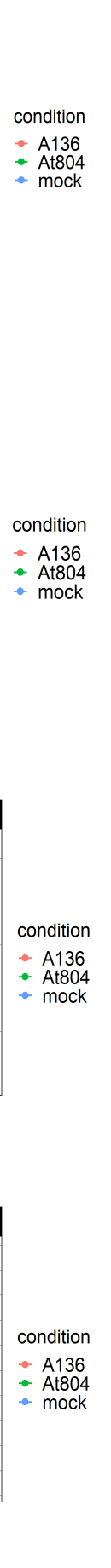
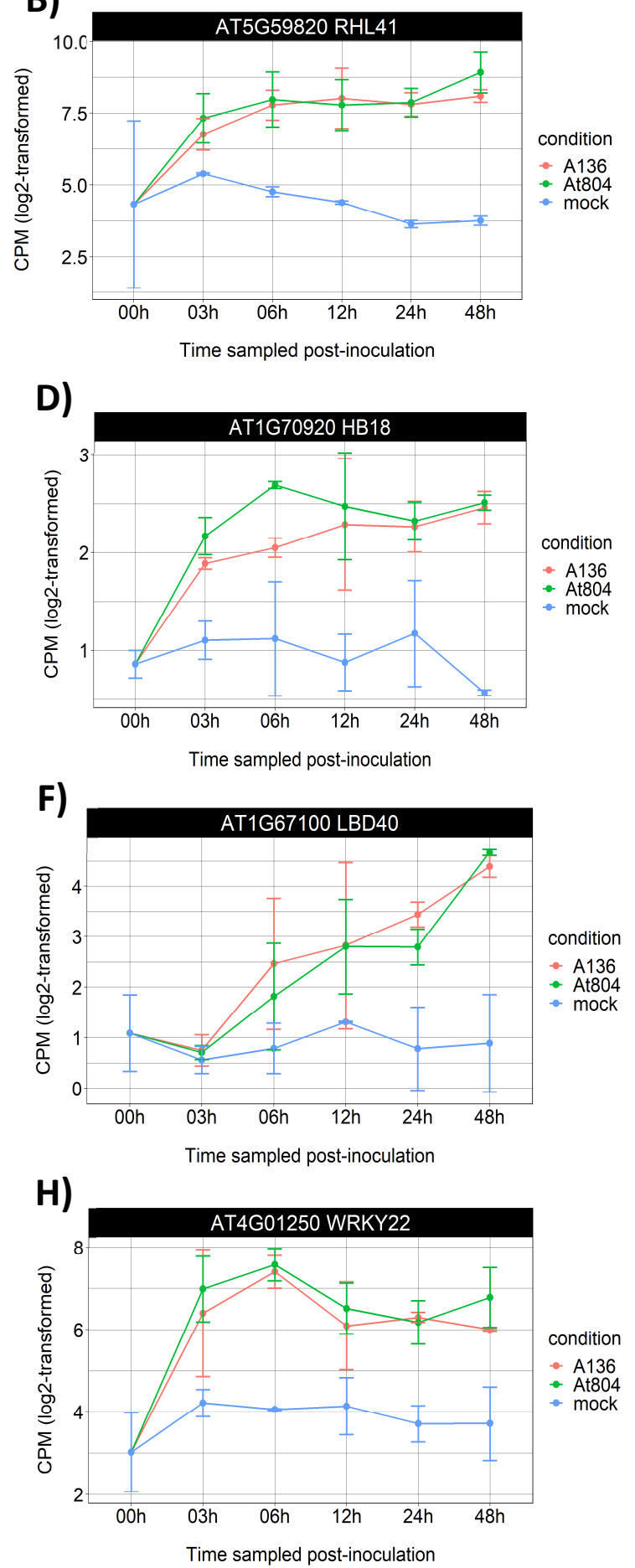

Figure 3.7: Expression of select transcription factor genes displaying clear upregulation

in both Agrobacterium treatments relative to the mock. A) AT1G22810, an unnamed 
member of the AP2/ERF transcription factor family that is upregulated early following inoculation before dropping back to baseline in the latter time-points; B) the $\mathrm{C} 2 \mathrm{H} 2$ family transcription factor RESPONSIVE TO HIGH LIGHT 41 (RHL41/ZAT12); C) the GRAS family transcription factor SCARECROW-like 14 (SCL14); D) the Arabidopsis HD-ZIP transcription factor homeobox-leucine zipper protein 18 (HB18); E) heat shock transcription factor $B 2 A(H S F B 2 A) ; F)$ the Lateral Organ Boundaries transcription factor LOB domain-containing protein 40 (LBD4O); G) the MYB-R2R3 transcription factor MYB DOMAIN PROTEIN 15 (MYB15); and $\mathbf{H}$ ) the WRKY family transcription factor WRKY22. 

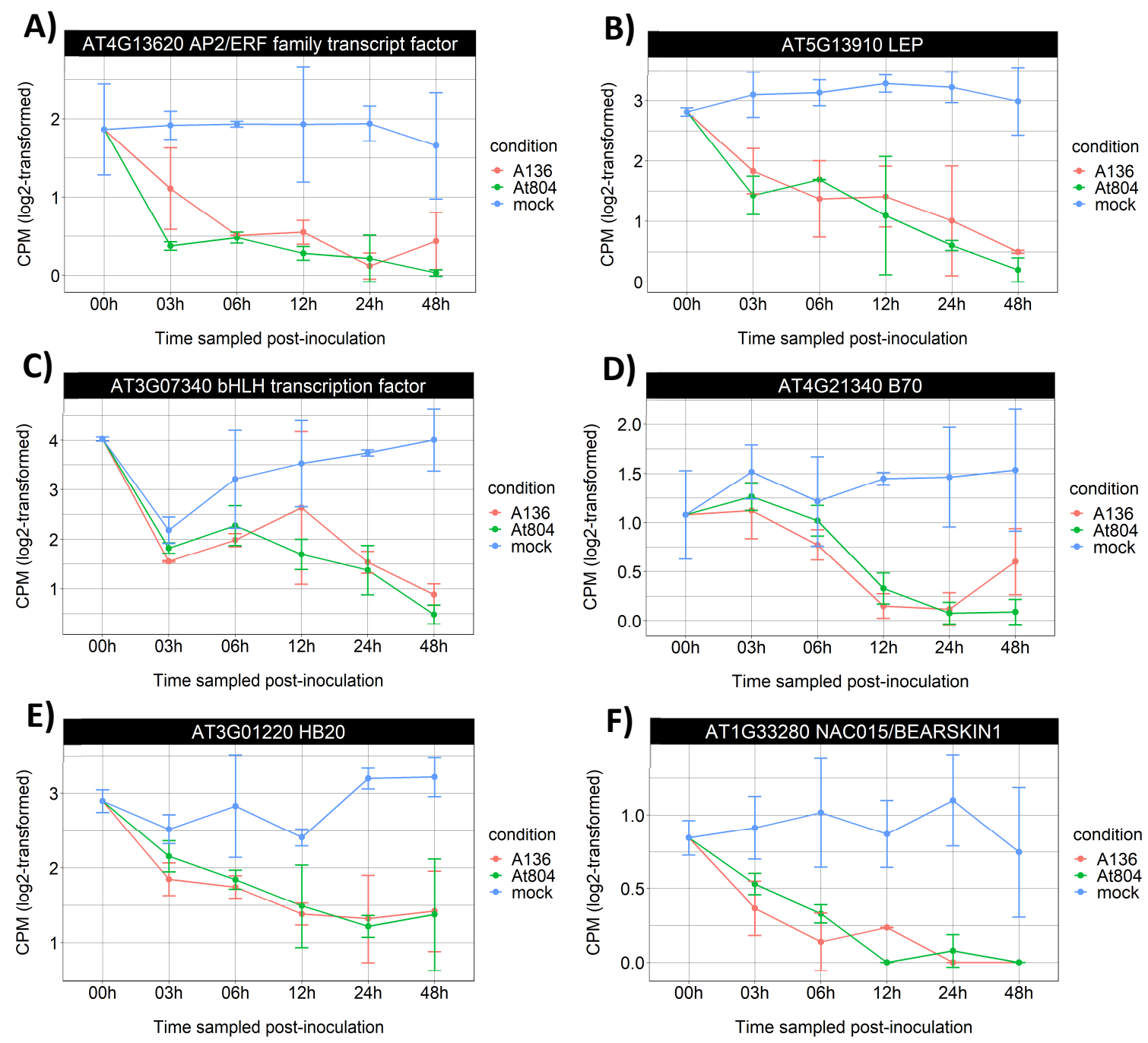

Figure 3.8: Expression of select transcription factor genes displaying clear downregulation in both Agrobacterium treatments relative to the mock. A) AT4G13620, an unnamed AP2/ERF transcription factor; B) another AP2/ERF transcription factor LEAFY PETIOLE (LEP); C) AT3G07340, an unnamed bHLH family transcription factor; D) another member of the bHLH transcription factor family named B70; E) the homeodomain-containing protein homeobox protein 20 (HB20); and F) the NAC family transcription factor NAC015/BEARSKIN1. 

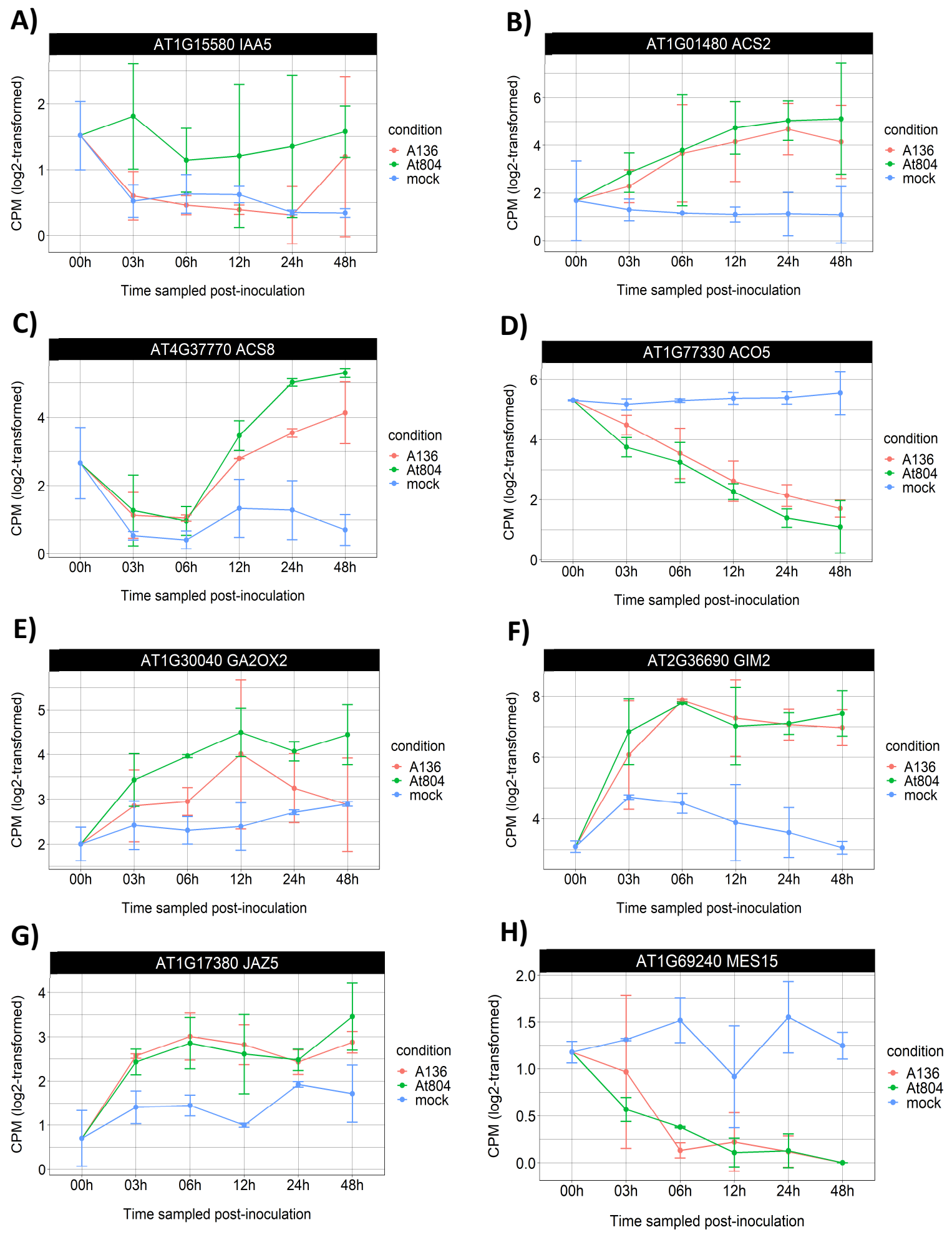

Figure 3.9: Select differentially expressed genes related to the hormones auxin, eth-

ylene, gibberellin, or jasmonic acid. A) The transcriptional co-repressor gene indole-3- 
acetic acid inducible 5 (IAA5), usually induced in response to auxin, was upregulated in the virulent vs. mock comparison at multiple time-points; B) and C) the ethylene biosynthesis genes 1-AMINOCYCLOPROPANE-1-CARBOXYLATE SYNTHASE 2 and 8 (ACS2 and ACS8), respectively, were both upregulated over time relative to the mock treatment for both Agrobacterium treatments; D) an ethylene biosynthesis gene (catalyzing a later step in the biosynthetic process than the one catalyzed by ACS) 1-AMINOCYCLOPROPANE-1-CARBOXYLATE OXIDASE 5 (ACO5) was downregulated over time in both the virulent and avirulent treatments relative to mock. E) the gibberellin metabolizing enzyme gene GIBBERELLIN 2-OXIDASE 2 (GA2OX2) was upregulated in the virulent vs. mock comparison at multiple time-points. F) the dioxygenase enzyme germination insensitive to ABA mutant 2 (GIM2), which is a regulator of gibberellin metabolism, was upregulated at all time-points past 6 hours in both comparisons. G) The transcriptional repressor involved in jasmonic acid signaling jasmonate-zim-domain protein 5 (JAZ5) was upregulated at multiple time-points in both comparisons. H) The METHYL ESTERASE 15/ROOT HAIR SPECIFIC 9 (MES15/RHS9) gene, which is putatively involved in catabolism of methyl jasmonate was downregulated at multiple time-points in both the Agrobacterium treatments compared to mock. 

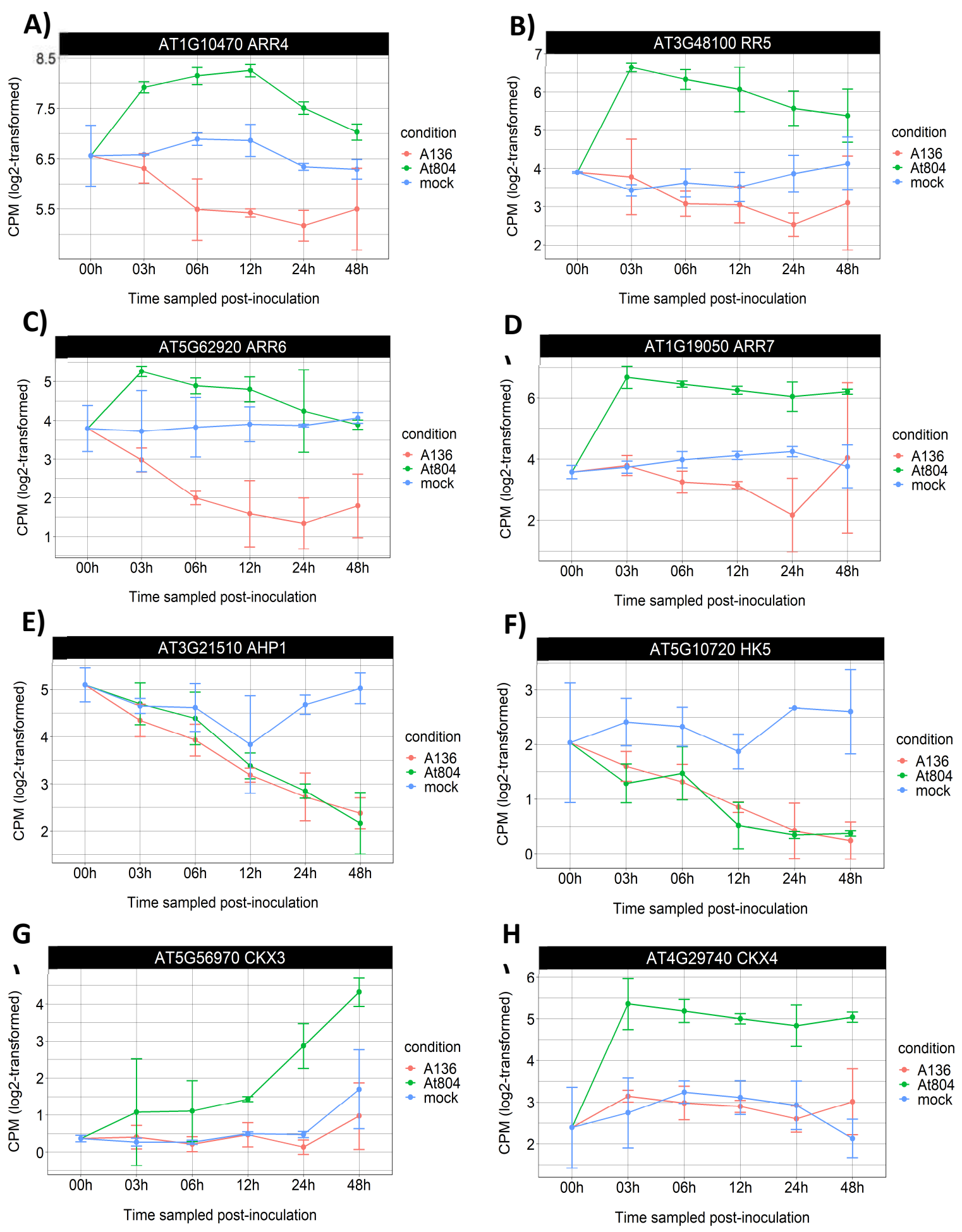

Figure 3.10: Select differentially expressed genes related to cytokinin. A-D) the type A ARABIDOPSIS RESPONCE REGULATOR (ARR) genes ARR4, 5, 6, and 7, respectively, which 
are negative regulators of cytokinin signaling, were the only differentially expressed in the virulent vs. avirulent comparison at the 6,12, and 24-hour time-points. ARR4 and ARR6 were significantly downregulated in the avirulent vs. mock comparison. E) Arabidopsis thaliana histidine phosphotransfer protein 1 (AHP1), a relay component in the cytokinin signaling pathway, was downregulated at the latter two time-points in both Agrobacterium to mock treatment comparisons as was another cytokinin phosphorelay signaling gene, F), Arabidopsis thaliana histidine kinase 5 (AHK5). G) and $\mathbf{H}$ ) genes coding for cytokinin catabolic enzymes, cytokinin oxidase 3 and 4 (CKX3 and 4), respectively, were both upregulated only in the virulent vs. mock comparison. CKX4 was upregulated beginning at an earlier time-point that $C K X 3$. 

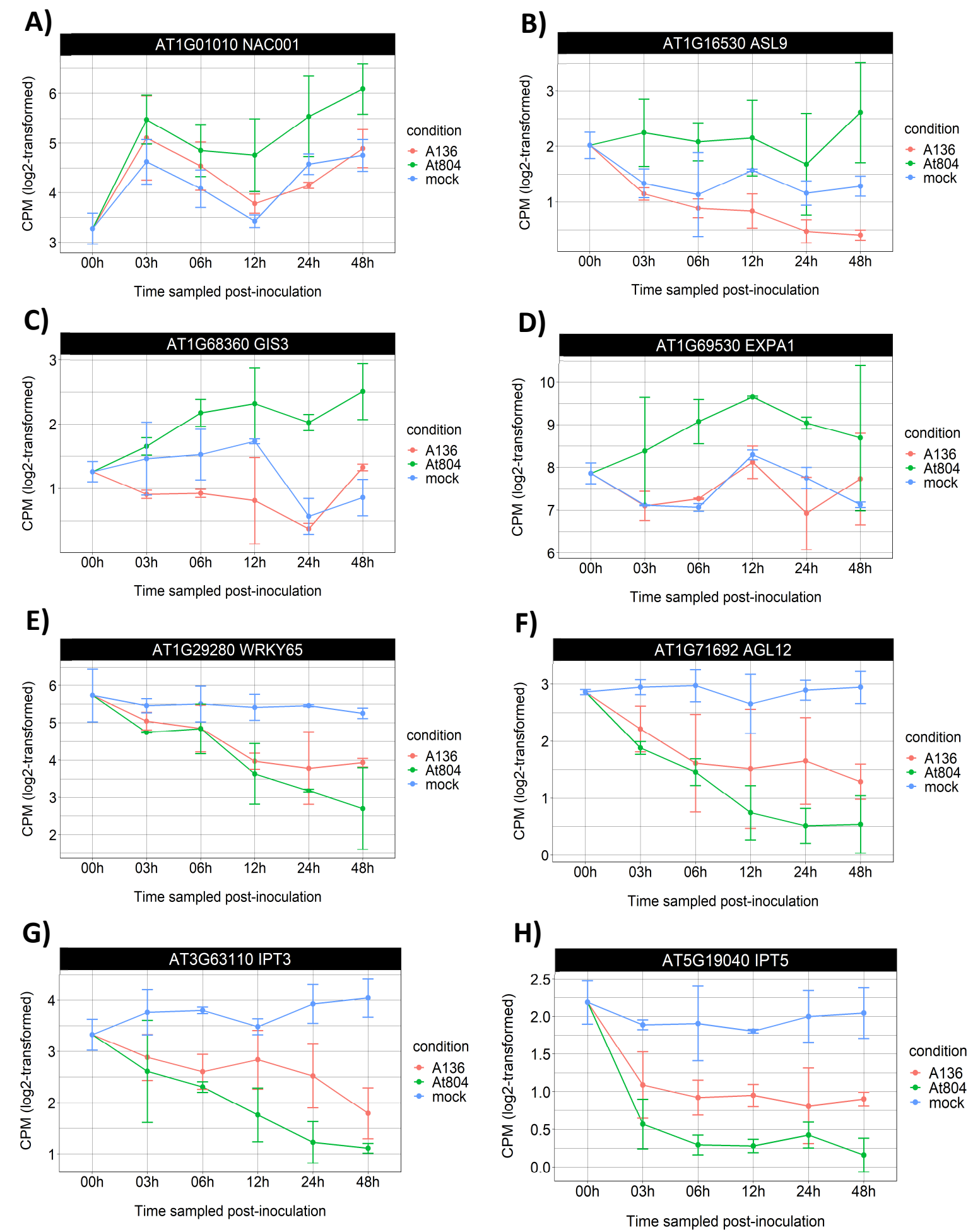

Figure 3.11: Genes differentially expressed only in the virulent vs. mock comparison showing apparent differences in expression trends between the virulent and avirulent 
treatments. A) The NAC family transcription factor NACO01, was upregulated in the virulent vs. mock comparison at 12, 24, and 48 hours post-inoculation. B) The Lateral Organ Boundaries domain transcription factor ASYMMETRIC LEAVES 2-like 9 (ASL9) was one of only two genes called as upregulated in the virulent vs. avirulent comparison at the 48hour time-point. However, it also showed an expression trend where levels were consistently lower than the mock treatment at all time-points in the avirulent treatment, and levels were highest in the virulent treatment at all times. C) The $\mathrm{C} 2 \mathrm{H} 2$ family transcription factor GLABROUS INFLORESCENCE STEMS3 (GIS3) also showed lower levels in the avirulent treatment at most time-points while the highest levels were seen in the virulent treatment. D) The cell wall metabolic protein EXPANSIN 1 (EXPA1) was differentially expressed in the virulent vs. mock comparison at the 6,12 , and 24-hour timepoint, while at the 12 and 24-hour points the expression levels in the avirulent treatment were lower than for mock. E) The WRKY65 transcription factor was downregulated in the virulent vs. mock comparison at 12,24 , and 48 hours post-inoculation, while higher levels were seen in the avirulent treatment at these times, whereas the same was true for $\mathbf{F}$ ), the MIKC/MADS-box transcription factor AGAMOUS-like 12 (AGL12). G) and $\mathbf{H}$ ) The cytokinin biosynthesis genes isopentenyltransferase 3 and 5 (IPT3 and 5), respectively, showed consistently lower expression levels in the virulent treatment than in the avirulent treatment through time, though both were lower in the mock treatment. 

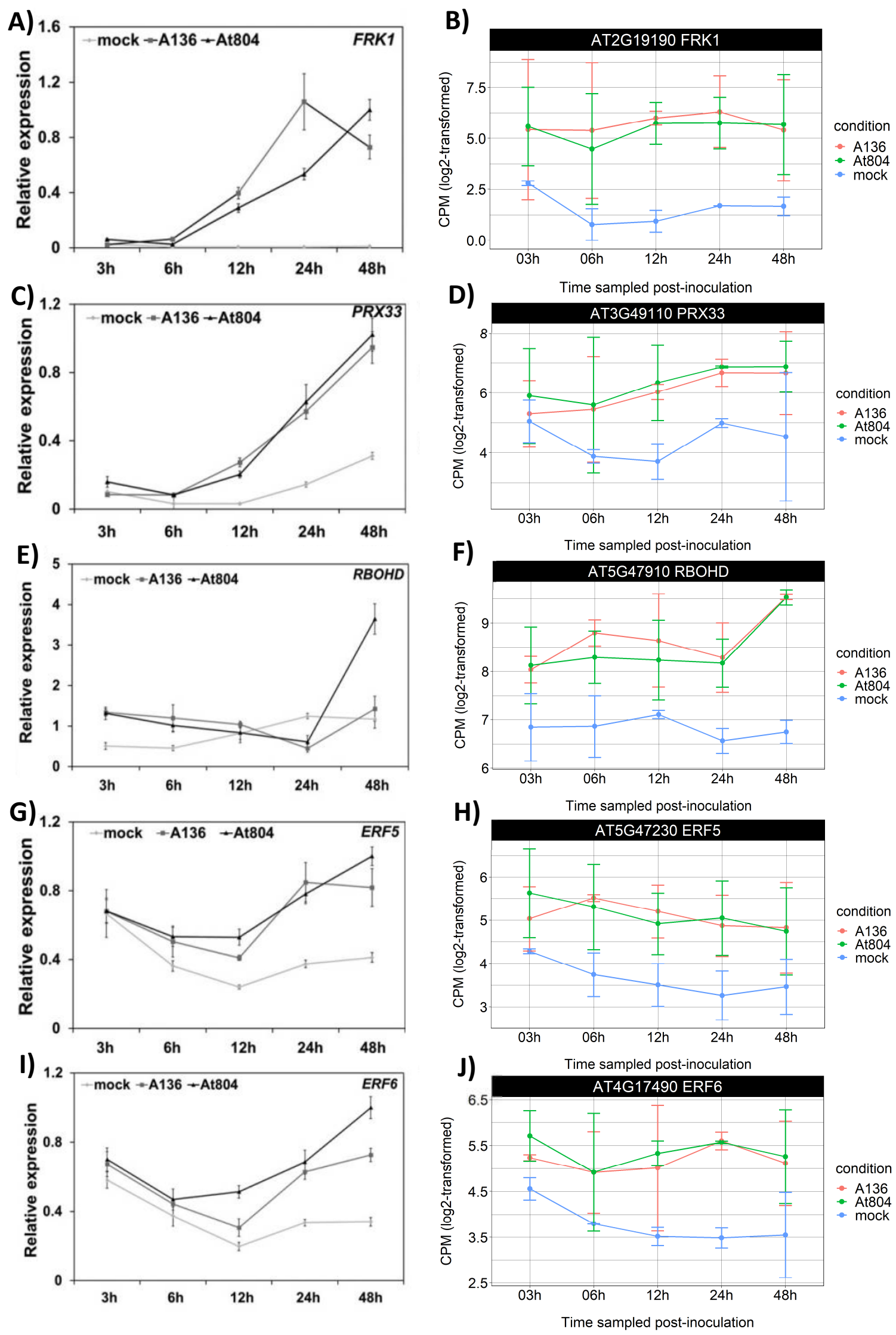
Figure 3.12: Relative expression for select genes quantified by qRT-PCR (A, C, E, G, I) juxtaposed with expression levels for the same gene as determined by RNA-seq (B, D, F, H, J). A-B) FLG22-induced receptor-like kinase 1 (FRK1); C-D) PEROXIDASE 33 (PRX33); EF) RESPIRATORY BURST OXIDASE HOMOLOGUE D (RBOHD); G-H) ETHYLENE RESPONSIVE ELEMENT BINDING FACTOR 5 (ERF5); I-J) ETHYLENE RESPONSIVE ELEMENT BINDING FACTOR 6 (ERF6). 
Table 3.1: Summary of RNA-seq data analysis

\begin{tabular}{|c|c|c|c|c|c|c|}
\hline sample & $\begin{array}{l}\text { total raw } \\
\text { reads }\end{array}$ & $\begin{array}{l}\text { total reads } \\
\text { remaining } \\
\text { after trim- } \\
\text { ming }\end{array}$ & $\begin{array}{l}\text { total reads } \\
\text { remaining } \\
\text { after clean- } \\
\text { ing }\end{array}$ & $\begin{array}{l}\text { reads } \\
\text { aligning } \\
\text { uniquely }\end{array}$ & $\begin{array}{l}\text { unique } \\
\text { alignments } \\
\text { assigned to } \\
\text { a gene }\end{array}$ & $\begin{array}{l}\text { percentage } \\
\text { of raw reads } \\
\text { used in DE } \\
\text { analysis }\end{array}$ \\
\hline mock_0h_rep1 & $7,963,574$ & $7,624,588$ & $7,556,870$ & $7,273,208$ & $7,042,927$ & $88.44 \%$ \\
\hline mock_0h_rep2 & $41,921,220$ & $40,543,657$ & $40,284,095$ & $38,624,547$ & $37,380,710$ & $89.17 \%$ \\
\hline mock_3h_rep1 & $9,472,498$ & $9,115,807$ & $9,065,824$ & $8,764,680$ & $8,489,023$ & $89.62 \%$ \\
\hline mock_3h_rep2 & $30,474,681$ & $29,442,244$ & $29,323,621$ & $28,525,019$ & $27,719,831$ & $90.96 \%$ \\
\hline mock_6h_rep1 & $7,607,790$ & $7,315,821$ & $7,239,600$ & $6,916,306$ & $6,678,814$ & $87.79 \%$ \\
\hline mock_6h_rep2 & $29,454,587$ & $28,454,968$ & $28,347,357$ & $27,605,264$ & $26,930,026$ & $91.43 \%$ \\
\hline mock_12h_rep1 & $9,518,182$ & $9,044,593$ & $9,016,830$ & $8,765,280$ & $8,488,899$ & $89.19 \%$ \\
\hline mock_12h_rep2 & $36,388,603$ & $35,180,377$ & $35,017,052$ & $33,889,748$ & $32,978,127$ & $90.63 \%$ \\
\hline mock_24h_rep1 & $9,063,056$ & $8,717,447$ & $8,674,953$ & $8,403,057$ & $8,134,675$ & $89.76 \%$ \\
\hline mock_24h_rep2 & $38,146,611$ & $36,901,907$ & $36,805,380$ & $35,828,917$ & $34,893,097$ & $91.47 \%$ \\
\hline mock_48h_rep1 & $8,414,022$ & $8,100,327$ & $8,032,850$ & $7,732,089$ & $7,477,149$ & $88.87 \%$ \\
\hline mock_48h_rep2 & $45,891,544$ & $44,419,279$ & $44,070,015$ & $41,219,991$ & $39,746,690$ & $86.61 \%$ \\
\hline A136_3h_rep1 & $8,976,836$ & $8,609,701$ & $8,566,894$ & $8,313,827$ & $8,039,362$ & $89.56 \%$ \\
\hline A136_3h_rep2 & $29,874,652$ & $28,784,177$ & $28,657,021$ & $27,848,195$ & $26,942,746$ & $90.19 \%$ \\
\hline A136_6h_rep1 & $8,638,554$ & $8,314,457$ & $8,217,534$ & $7,869,222$ & $7,606,868$ & $88.06 \%$ \\
\hline A136_6h_rep2 & $26,153,081$ & $25,290,373$ & $25,197,862$ & $24,594,804$ & $23,818,433$ & $91.07 \%$ \\
\hline A136_12h_rep1 & $7,640,701$ & $7,366,668$ & $7,304,009$ & $6,994,814$ & $6,752,795$ & $88.38 \%$ \\
\hline A136_12h_rep2 & $32,754,109$ & $31,578,673$ & $31,359,706$ & $30,295,378$ & $29,270,583$ & $89.36 \%$ \\
\hline A136_24h_rep1 & $7,082,774$ & $6,807,942$ & $6,739,561$ & $6,472,907$ & $6,246,961$ & $88.20 \%$ \\
\hline A136_24h_rep2 & $32,168,377$ & $31,037,986$ & $30,697,768$ & $29,289,158$ & $28,254,059$ & $87.83 \%$ \\
\hline A136_48h_rep1 & $8,796,410$ & $8,460,645$ & $8,400,209$ & $8,114,346$ & $7,845,389$ & $89.19 \%$ \\
\hline A136_48h_rep2 & $28,254,093$ & $27,238,222$ & $26,979,862$ & $25,495,300$ & $24,477,204$ & $86.63 \%$ \\
\hline At804_3h_rep1 & $9,370,855$ & $8,973,319$ & $8,919,575$ & $8,649,398$ & $8,374,975$ & $89.37 \%$ \\
\hline At804_3h_rep2 & $31,850,786$ & $30,790,202$ & $30,653,222$ & $29,842,519$ & $28,896,941$ & $90.73 \%$ \\
\hline At804_6h_rep1 & $8,065,451$ & $7,749,987$ & $7,684,386$ & $7,416,182$ & $7,186,921$ & $89.11 \%$ \\
\hline At804_6h_rep2 & $32,600,304$ & $31,485,535$ & $31,306,547$ & $30,419,497$ & $29,412,528$ & $90.22 \%$ \\
\hline At804_12h_rep1 & $7,829,792$ & $7,514,508$ & $7,466,606$ & $7,206,366$ & $6,985,901$ & $89.22 \%$ \\
\hline At804_12h_rep2 & $31,462,402$ & $30,405,682$ & $30,246,360$ & $29,363,771$ & $28,461,715$ & $90.46 \%$ \\
\hline At804_24h_rep1 & $10,590,186$ & $10,188,551$ & $10,155,954$ & $9,894,166$ & $9,590,388$ & $90.56 \%$ \\
\hline At804_24h_rep2 & $34,156,011$ & $32,979,341$ & $32,682,786$ & $31,280,047$ & $30,268,847$ & $88.62 \%$ \\
\hline At804_48h_rep1 & $7,675,826$ & $7,331,672$ & $7,263,071$ & $6,933,108$ & $6,701,024$ & $87.30 \%$ \\
\hline At804_48h_rep2 & $32,929,047$ & $31,803,912$ & $31,548,015$ & $30,187,970$ & $29,149,703$ & $88.52 \%$ \\
\hline
\end{tabular}


Table 3.2: Highly upregulated genes in avirulent vs. mock and virulent vs. mock comparisons through all times

\begin{tabular}{|c|c|c|c|}
\hline Gene ID & Gene Name & Description & Contrasts in which gene was top 10 downregulated ( $\log _{2}$-fold change) \\
\hline AT4G30380 & - & Barwin-related endoglucanase & V-M03 (7.61) \\
\hline AT1G22810 & - & Integrase-type DNA-binding superfamily protein & V-M03 (5.67); A-M06 (6.14); V-M06 (6.24) \\
\hline AT1G52560 & - & HSP20-like chaperones superfamily protein & A-M03 (5.53); V-M03 (4.72); A-M06 (5.60); A-M24 (7.95) \\
\hline AT5G12020 & HSP17.6II & $17.6 \mathrm{kDa}$ class II heat shock protein & $\mathrm{A}-\mathrm{M} 03$ (4.26); A-M12 (7.86); V-M12 (7.71) \\
\hline AT2G29500 & - & HSP20-like chaperones superfamily protein & A-M03 (4.06); V-M03 (3.10) \\
\hline AT3G46230 & HSP17.4 & heat shock protein 17.4 & A-M03 (4.02); A-M24 (8.13) \\
\hline AT5G12030 & HSP17.6A & heat shock protein $17.6 \mathrm{~A}$ & A-M03 (3.95) \\
\hline AT5G39580 & - & Peroxidase superfamily protein & A-M03 (3.61) \\
\hline AT2G43620 & - & Chitinase family protein & A-M03 (3.52); V-M03 (3.09) \\
\hline AT2G39200 & MLO12 & Seven transmembrane MLO family protein & A-M03 (3.49); V-M03 (3.56) \\
\hline AT3G48100 & RR5 & response regulator 5 & V-M03 (3.42) \\
\hline AT1G19050 & ARR7 & response regulator 7 & V-M03 (3.14) \\
\hline AT4G01250 & WRKY22 & WRKY family transcription factor & V-M03 (3.03) \\
\hline AT4G27450 & - & aluminum induced protein with YGL and LRDR motifs & V-M03 (3.03) \\
\hline AT2G14610 & PR1 & pathogenesis-related protein 1 & A-M06 (15.17); V-M06 (15.03) \\
\hline AT3G29970 & - & B12D protein & $\begin{array}{l}\text { A-M06 (10.36); V-M06 (9.82); A-M12 (7.86); V-M12 (8.34); A-M24 } \\
\text { (9.65); V-M24 (9.77) }\end{array}$ \\
\hline AT4G37710 & VQ29 & VQ motif-containing protein & A-M06 (8.57); V-M06 (8.27); A-M24 (10.15); V-M24 (9.72) \\
\hline AT5G25260 & - & $\begin{array}{l}\text { SPFH/Band 7/PHB domain-containing membrane-associated pro- } \\
\text { tein family }\end{array}$ & V-M06 (8.35); A-M12 (9.32); V-M12 (9.56) \\
\hline AT4G11170 & RMG1 & Disease resistance protein (TIR-NBS-LRR class) family & A-M06 (8.23); V-M06 (7.14) \\
\hline AT5G55150 & - & F-box SKIP23-like protein (DUF295) & V-M06 (7.43); V-M48 (12.60) \\
\hline AT2G19190 & FRK1 & FLG22-induced receptor-like kinase 1 & A-M06 (7.06) \\
\hline AT3G28570 & - & $\begin{array}{l}\text { P-loop containing nucleoside triphosphate hydrolases superfamily } \\
\text { protein }\end{array}$ & V-M06 (6.64) \\
\hline AT4G10250 & ATHSP22.0 & HSP20-like chaperones superfamily protein & $\begin{array}{l}\text { A-M06 (6.35); V-M06 (6.37); A-M12 (8.23); V-M12 (8.41); A-M24 } \\
\text { (11.22); V-M24 (10.70); A-M48 (9.33) }\end{array}$ \\
\hline
\end{tabular}




\begin{tabular}{|c|c|c|c|}
\hline AT4G27670 & HSP21 & heat shock protein 21 & A-M06 (5.81); V-M06 (6.21); A-M48 (9.48) \\
\hline AT1G21240 & WAK3 & wall associated kinase 3 & V-M12 (10.17) \\
\hline AT4G03450 & - & Ankyrin repeat family protein & A-M12 (9.87) \\
\hline AT1G32350 & AOX1D & alternative oxidase 1D & $\begin{array}{l}\text { A-M12 (9.24); V-M12 (8.82); A-M24 (10.32); V-M24 (10.31); A-M48 } \\
\text { (10.22); V-M48 (12.04) }\end{array}$ \\
\hline AT2G36020 & HVA22J & HVA22-like protein J & A-M12 (8.60) \\
\hline AT5G51465 & - & hypothetical protein & A-M12 (8.23); V-M12 (8.05) \\
\hline AT1G68765 & IDA & Putative membrane lipoprotein & V-M12 (8.03); V-M48 (11.12) \\
\hline AT1G05880 & ARI12 & RING/U-box superfamily protein & V-M12 (8.02) \\
\hline AT2G33780 & - & VQ motif-containing protein & V-M12 (8.00); V-M24 (8.23) \\
\hline AT1G21940 & - & transmembrane protein & A-M12 (7.28) \\
\hline AT1G53540 & - & HSP20-like chaperones superfamily protein & A-M12 (7.27) \\
\hline AT1G26390 & - & FAD-binding Berberine family protein & A-M24 (9.44); V-M24 (9.42) \\
\hline AT1G69930 & GSTU11 & glutathione S-transferase TAU 11 & A-M24 (9.42); V-M24 (9.37) \\
\hline AT3G54520 & - & hypothetical protein & V-M24 (8.27); V-M48 (10.86) \\
\hline AT2G23270 & - & transmembrane protein & V-M24 (8.22); A-M48 (9.84); V-M48 (11.49) \\
\hline AT4G26260 & MIOX4 & myo-inositol oxygenase 4 & V-M24 (8.22) \\
\hline AT4G31970 & CYP82C2 & cytochrome P450, family 82 , subfamily C, polypeptide 2 & A-M24 (8.12); A-M48 (11.75); V-M48 (10.98) \\
\hline AT2G17740 & - & Cysteine/Histidine-rich C1 domain family protein & A-M24 (8.12) \\
\hline AT1G21850 & sks8 & SKU5 similar 8 & V-M48 (11.61) \\
\hline AT4G28460 & - & transmembrane protein & V-M48 (11.05) \\
\hline AT3G54530 & - & hypothetical protein & V-M48 (10.39) \\
\hline AT5G64790 & - & O-Glycosyl hydrolases family 17 protein & A-M48 (9.51); V-M48 (10.59) \\
\hline AT4G37290 & - & transmembrane protein & A-M48 (9.48) \\
\hline AT1G66570 & SUC7 & sucrose-proton symporter 7 & A-M48 (9.44) \\
\hline AT5G36970 & NHL25 & NDR1/HIN1-like 25 & A-M48 (9.14) \\
\hline AT3G53600 & - & $\mathrm{C} 2 \mathrm{H} 2$-type zinc finger family protein & A-M48 (9.08) \\
\hline
\end{tabular}


Table 3.3: Highly downregulated genes in avirulent vs. mock and virulent vs. mock comparisons through all times

\begin{tabular}{|c|c|c|c|}
\hline Gene ID & Gene Name & Description & Contrasts in which gene was top 10 downregulated ( $\log _{2}$-fold change) \\
\hline AT3G62270 & BOR2 & HCO3- transporter family & V-M03 (-2.48) \\
\hline AT1G54970 & PRP1 & proline-rich protein 1 & A-M06 (-7.49); V-M06 (-5.18); A-M12 (-6.49); A-M24 (-7.90) \\
\hline AT4G31470 & - & $\begin{array}{l}\text { CAP (Cysteine-rich secretory proteins, Antigen } 5 \text {, and Patho- } \\
\text { genesis-related } 1 \text { protein) superfamily protein }\end{array}$ & A-M06 (-5.45); A-M24 (-6.98) \\
\hline AT1G01580 & FRO2 & ferric reduction oxidase 2 & V-M06 (-4.84) \\
\hline AT1G19900 & - & glyoxal oxidase-related protein & A-M06 (-4.80); A-M12 (-6.92); V-M12 (-6.91); A-M24 (-6.96) \\
\hline AT5G26310 & UGT72E3 & UDP-Glycosyltransferase superfamily protein & A-M06 (-4.72); V-M12 (-7.07) \\
\hline AT2G23630 & sks16 & SKU5 similar 16 & V-M06 (-4.51); V-M24 (-8.25); A-M48 (-8.37) \\
\hline AT5G20860 & - & Plant invertase/pectin methylesterase inhibitor superfamily & A-M06 (-4.49) \\
\hline AT5G55110 & - & Stigma-specific Stig1 family protein & V-M06 (-4.42) \\
\hline AT5G60520 & - & Late embryogenesis abundant (LEA) protein-like protein & V-M06 (-4.36); V-M24 (-7.84) \\
\hline AT4G08410 & - & Proline-rich extensin-like family protein & A-M06 (-4.28) \\
\hline AT4G37160 & sks15 & SKU5 similar 15 & V-M06 (-4.19); V-M24 (-8.84) \\
\hline AT5G04960 & - & Plant invertase/pectin methylesterase inhibitor superfamily & A-M06 (-4.10); V-M06 (-5.05); A-M12 (-6.37); V-M24 (-7.99); V-M48 (-9.36) \\
\hline AT1G48930 & GH9C1 & glycosyl hydrolase $9 \mathrm{C1}$ & A-M06 (-3.99) \\
\hline AT1G12040 & LRX1 & leucine-rich repeat/extensin 1 & A-M06 (-3.91); V-M12 (-7.41); V-M48 (-9.20) \\
\hline AT1G49450 & - & Transducin/WD40 repeat-like superfamily protein & A-M06 (-3.79) \\
\hline AT5G06630 & - & proline-rich extensin-like family protein & V-M06 (-3.70) \\
\hline AT5G10130 & - & Pollen Ole e 1 allergen and extensin family protein & V-M06 (-3.42) \\
\hline AT1G30870 & - & Peroxidase superfamily protein & V-M06 (-3.32); A-M48 (-8.41) \\
\hline AT3G19430 & - & $\begin{array}{l}\text { late embryogenesis abundant protein-related / LEA protein- } \\
\text { like protein }\end{array}$ & V-M12 (-8.39); V-M24 (-8.68) \\
\hline AT5G06640 & EXT10 & Proline-rich extensin-like family protein & $\mathrm{A}-\mathrm{M} 12(-7.87)$ \\
\hline AT4G12520 & - & $\begin{array}{l}\text { Bifunctional inhibitor/lipid-transfer protein/seed storage } 2 \mathrm{~S} \\
\text { albumin superfamily protein }\end{array}$ & A-M12 (-7.75) \\
\hline AT2G24980 & EXT6 & Proline-rich extensin-like family protein & V-M12 (-7.64) \\
\hline AT2G33790 & AGP30 & arabinogalactan protein 30 & V-M12 (-7.55); A-M24 (-6.97); V-M48 (-9.39) \\
\hline
\end{tabular}




\begin{tabular}{|c|c|c|c|}
\hline AT3G62680 & PRP3 & proline-rich protein 3 & V-M12 (-7.53); A-M48 (-9.75) \\
\hline AT5G57530 & XTH12 & xyloglucan endotransglucosylase/hydrolase 12 & V-M12 (-7.26) \\
\hline AT3G49960 & - & Peroxidase superfamily protein & A-M12 (-6.92) \\
\hline AT1G62980 & EXPA18 & expansin A18 & V-M12 (-6.86) \\
\hline AT5G35190 & EXT13 & proline-rich extensin-like family protein & A-M12 (-6.85) \\
\hline AT5G54020 & - & Cysteine/Histidine-rich C1 domain family protein & A-M12 (-6.72) \\
\hline AT5G04970 & - & Plant invertase/pectin methylesterase inhibitor superfamily & A-M12 (-6.42); V-M12 (-7.26) \\
\hline AT2G32620 & CSLB02 & cellulose synthase-like B & A-M12 (-6.33) \\
\hline AT5G54370 & - & Late embryogenesis abundant (LEA) protein-like protein & V-M24 (-8.75) \\
\hline AT3G54590 & HRGP1 & hydroxyproline-rich glycoprotein & V-M24 (-8.27); A-M48 (-8.69) \\
\hline AT1G47600 & BGLU34 & beta glucosidase 34 & V-M24 (-7.98) \\
\hline AT3G59930 & - & defensin-like protein & A-M24 (-7.93); A-M48 (-8.57) \\
\hline AT5G62340 & - & $\begin{array}{l}\text { Plant invertase/pectin methylesterase inhibitor superfamily } \\
\text { protein }\end{array}$ & V-M24 (-7.90); A-M48 (-8.39) \\
\hline AT1G05660 & - & Pectin lyase-like superfamily protein & A-M24 (-7.03) \\
\hline AT3G01260 & - & Galactose mutarotase-like superfamily protein & A-M24 (-6.98) \\
\hline AT1G50060 & - & $\begin{array}{l}\text { CAP (Cysteine-rich secretory proteins, Antigen } 5 \text {, and Patho- } \\
\text { genesis-related } 1 \text { protein) superfamily protein }\end{array}$ & A-M24 (-6.98) \\
\hline AT3G06460 & - & GNS1/SUR4 membrane protein family & A-M24 (-6.95) \\
\hline AT1G51470 & BGLU35 & beta glucosidase 35 & A-M24 (-6.87); V-M24 (-8.93) \\
\hline AT5G56080 & NAS2 & nicotianamine synthase 2 & A-M48 (-19.48); V-M48 (-19.70) \\
\hline AT3G28550 & - & Proline-rich extensin-like family protein & V-M48 (-11.65) \\
\hline AT3G01190 & - & Peroxidase superfamily protein & V-M48 (-10.97) \\
\hline AT1G66270 & BGLU21 & Glycosyl hydrolase superfamily protein & V-M48 (-10.67) \\
\hline AT1G52060 & - & Mannose-binding lectin superfamily protein & V-M48 (-10.15) \\
\hline AT4G11320 & $\mathrm{CP} 2$ & Papain family cysteine protease & V-M48 (-9.49) \\
\hline AT3G54580 & - & Proline-rich extensin-like family protein & A-M48 (-8.81); V-M48 (-10.68) \\
\hline AT1G23720 & - & Proline-rich extensin-like family protein & A-M48 (-8.68) \\
\hline AT5G15180 & PRX56 & Peroxidase superfamily protein & A-M48 (-8.40) \\
\hline
\end{tabular}




\section{Chapter 4}

\section{Evaluation of the influence of candidate genes on somatic embryogenesis using sorghum transformation}

Note: Muruganantham Mookkan assisted with fluorescent imaging of marker gene expression and particle bombardment experiments. All other work described in this chapter was performed by the author. 


\section{Summary}

Genetic engineering of most monocot crops depends on clonal plant regeneration through somatic embryogenesis. Crop biotechnology is limited by the fact that only relatively few genotypes of economically important species are highly regenerable through currently available tissue culture methods and are thus suitable for transformation. In recent years, genes that have been known to play major roles in somatic embryogenesis and other modes of regeneration have had major impacts on crop biotechnology by serving as tools that allow some of these barriers to be overcome, particularly in cereal crops. Several candidate genes, which were identified from the RNA-seq differential expression analysis described in chapter 2 as those potentially playing a role in governing the somatic embryogenesis process, were experimentally assessed through multiple transgenic approaches in sorghum. All of these experiments relied on comparing counts of somatic embryos formed in tissue culture from samples of explants transformed either with transgene cassettes meant to ectopically express or repress target gene transcription, or with an empty vector control. RNAi-mediated silencing constructs targeting individual genes were delivered into explants of two sorghum genotypes routinely used in sorghum transformation by either Agrobacterium-mediated transformation or particle bombardment. Transgene cassettes constitutively overexpressing the cDNA sequences for the maize orthologs of several sorghum candidate genes were transferred to explants of a recalcitrant sorghum inbred line, also via both of these transformation methods. Finally, a newly assembled dexamethasone-inducible expression construct was validated for expression efficiency of marker genes, and then used to 
deliver candidate gene sequences into sorghum explants under dexamethasone treatment. No statistically significant differences were seen between treatments in any of the experiments performed. Pitfalls in each of the experimental approaches which could have potentially confounded detection of candidate gene involvement in somatic embryogenesis are discussed.

\section{Introduction}

The complex developmental process of somatic embryogenesis is known to be controlled at multiple levels including through epigenetic modulation, key transcriptional regulators, and through hormone metabolism, transport, and signaling pathways (Horstman et al. 2017a). For biotechnological applications of somatic embryogenesis, artificial tissue culture conditions must be developed and optimized for individual species and, in many cases, individual genotypes (Smith 2012). Tissue culture variables that have been known to affect regeneration through somatic embryogenesis include pretreatment of plant tissue to induce stress response such as heat shock, osmotic shock, or mechanical wounding, carbon and nitrogen sources and gelling agent types used in media, and the light intensity, photoperiod, and temperature conditions under which cultures are incubated (Loyola-Vargas et al. 2008). However, the most significant parameter in the tissue culture procedure is often the application of exogenous plant growth regulators (PGRs) in media. Auxins are most commonly used to induce somatic embryo formation, though cytokinins, as well as abscisic acid and gibberellins may be used in some cases, sometimes in combination with auxins (Elhiti et al. 2013). Variation of the 
specific compound type used (e.g., IAA, NAA, or 2,4-D for auxin) and concentration frequently affects regeneration and thus several hormone treatments may be tested in protocol optimization experiments (Bidabadi and Jain 2020). Though many advances have been made in plant biotechnology over the last several decades through development of tissue culture methods, many plant species and genotypes remain infeasible to manipulate with genetic engineering due to a lack of established tissue culture protocols providing efficient regeneration (Yin et al. 2017).

A method that has shown promise for alleviating the constraints on regeneration in recent years involves the transgenic expression of developmental regulator genes (sometimes referred to as DRs, Dev genes, or morphogenic genes) known to play key roles in controlling different stages of somatic embryogenesis (Gordon-Kamm et al. 2019, Maher et al. 2020). One of the most notable examples of this was the application of the genes BABY BOOM (BBM) and WUSCHEL 2 (WUS2), which are involved in embryo induction and meristem establishment/organizing, respectively, to enhance transformation in maize and other monocots (Lowe et al. 2016, Mookkan et al. 2017). By confining the expression of each gene to the specific developmental stage and tissue context in which they are active in promoting somatic embryo formation, a series of transgene constructs were developed which were able to confer some level of increased somatic embryo generation, and hence transformation frequency, in each maize line they were deployed in (Lowe et al. 2018). Controlling the spatiotemporal distribution and strength of expression in these developmental regulators appears to be essential for utilizing 
them for practical transformation purposes as constitutive expression often leads to developmental abnormalities sometimes including infertility (Gordon-Kamm et al. 2019). Various methods for restricting gene expression such as use of tissue-specific promoters, excision of transgenes through site-specific recombinases, or inducible promoter systems have been useful when investigating these genes and may be deployed to help mitigate the potential for pleiotropic effects from these genes (Arroyo-Herrera et al. 2008, Deng et al. 2009, Gazzarrini et al. 2004).

There have been several plant genes other than $B B M$ and WUS which are known to influence somatic embryo formation. Some of these genes can only improve a preexisting embryogenesis culture response, such as SERK1, AGL15, or STM (Elhiti et al. 2010, Harding et al. 2003, Hecht et al. 2001), whereas others including EMK, RKD4, LEC1, LEC2 and PGA37 (Lotan et al. 1998, Mursyanti et al. 2015, Tsuwamoto et al. 2010, Wang et al. 2009, Wójcikowska et al. 2013) are capable of inducing somatic embryogenesis in tissue culture conditions where regeneration does not normally take place. The majority of studies characterizing the embryogenic function of these genes have been performed in the model plants Arabidopsis thaliana and Nicotiana tabacum. However, some have been carried out in crop plants such as LEC1-like (L1L) in citrus (Zhu et al. 2014), AGL15 in soybean (Thakare et al. 2008), and ZmHb2 (suppression) in maize (Huang et al. 2014). Activity of one gene in a certain plant does not necessarily translate between species. This comes into application with respect to monocots and dicots as there are morphological (and thus likely molecular) differences in somatic embryo development between 
plants from the two groups. Practical developmental regulator-based methods for enhancing transformation that work well in some monocots have generally not translated to dicot species where solutions of this nature, at least with respect to somatic embryogenesis, remain more elusive (Gordon-Kamm et al. 2019). Thus, uncovering additional molecular players in somatic embryogenesis could add to the toolkit of available developmental regulators for enhancing transformation in new species.

Based on candidate sequences identified from previously performed RNA-seq work in sorghum, this study sought to functionally characterize putative roles of these genes in altering the frequency of somatic embryo induction through transgenic testing in sorghum lines. Silencing of candidate genes by way of long-hairpin RNA interference (RNAi), which has been well-documented to consistently repress target gene expression in a variety of plant species and contexts (Matthew 2004, Small 2007), was used to search for transformant groups with decreased ability to form somatic embryos. Ectopic expression of sorghum candidate gene sequences (or their maize orthologs) through either strong, constitutive expression or conditional expression (chemically induced by dexamethasone) was used to examine potential increases in somatic embryo induction. By monitoring transformed explants over a period of 4-6 weeks in tissue culture, the frequency of regeneration via somatic embryogenesis could be assessed.

\section{Results}

Functional testing of candidate genes through sorghum transformation 
Using the differential gene expression analysis data described earlier in Chapter 2 , genes were selected from those that showed clear expression differences through multiple time-points between the [+] 2,4-D and the [-] 2,4-D conditions as candidates for potentially playing a role in regulating somatic embryogenesis in sorghum. To functionally validate these genes in sorghum, several transgenic strategies were employed following previously established transformation and tissue culture protocols and using multiple sorghum genotypes. Sorghum immature zygotic embryo explants ranging from 1.2 to $1.5 \mathrm{~mm}$ in size were isolated from seeds and were transformed with various transgene constructs by either Agrobacterium-mediated transformation or particle bombardment. Plant tissues were maintained and monitored in tissue culture for up to 2 months following transformation. Potential regulatory control over somatic embryogenesis could then tentatively be detected by comparing counts of regenerating somatic embryos from groups of explants transformed with these constructs to a group receiving an empty vector control treatment. Key aspects of the sorghum transformation and tissue culture regeneration process are described in Figure 4.1.

\section{Silencing of candidate genes using RNAi}

One strategy employed to attempt to capture the effect that candidate genes may have over regeneration was to silence their expression through transgenic delivery of long-hairpin RNAi constructs targeting individual genes. Segments of candidate gene cDNA sequences ranging from 300 to $600 \mathrm{bp}$ in length containing multiple predicted small RNA sequences potentially targeting the gene of interest were selected for DNA 
synthesis. See Figure 4.6 for the nucleotide sequences of the RNAi segments used for each candidate gene in this study along with the restriction enzyme sites used for vector assembly. These sequences were then inserted as pairs of inverted repeats (separated by a plant-active intron sequence) into the backbone of the binary vector pFGC5941, which has been previously shown to confer high rates of transgene expression in sorghum (Do et al. 2016), via restriction cloning and ligation. In total, 10 RNAi plasmids were assembled, each targeting an individual candidate gene. Figure 4.5A \& B display plasmid maps of the original pFGC5941 vector and a representative RNAi expression plasmid, respectively.

After mobilizing each plasmid to cells of the Agrobacterium tumefaciens strain $A G L 1$, the resulting isolates were used for transformation of immature zygotic embryos of the highly regenerable sorghum genotype P898012 following the protocol outlined in Do et al. (2016) in three separate experiments testing 3-4 RNAi plasmids each alongside empty pFGC5941 as a negative control. These experiments were each repeated in triplicate using seeds derived from separate sorghum grain heads. Results of the first, second, and third RNAi experiments in P898012 are summarized in Tables 4.1, 4.3, and 4.4, respectively. Since most of the candidate genes tested in this experiment were strongly upregulated in the [+] 2,4-D condition relative to the [-] 2,4-D condition in the RNA-seq data, it was anticipated that silencing of genes that, in fact, control somatic embryogenesis should decrease the number of somatic embryos regenerated relative to the control treatment. One-way ANOVA testing in each experiment revealed no significant differences at $\alpha=0.05$ between treatments in either the percentage of explants forming calli 
or the number of total somatic embryos formed over the course of four weeks in tissue culture. The lowest $P$-value calculated for analysis of the three experiments was 0.286 for experiment 2 .

To test RNAi constructs by a different transformation method in a different genetic background, pre-cultured immature embryos of another highly regenerable sorghum genotype, Tx430, were transformed with plasmid DNA via particle bombardment following the protocol described in Liu et al. (2014). RNAi expression plasmids for targeting five different IAA candidate genes were tested by this method. Since one possible reason for the failure of silencing individual genes to show an effect on somatic embryogenesis could be functional redundancy, one treatment in this experiment was co-bombarded with all five constructs by mixing them in equimolar quantities prior to gold particle coating. Regenerating somatic embryos were again counted over the next four weeks in tissue culture. Again, no significant differences were seen between treatments by one-way ANOVA. Results of this experiment are summarized in Table 4.5.

Constitutive overexpression of maize orthologs of candidate genes in a non-regenerable sorghum genotype

The next effort made to assay the impact of candidate genes on somatic embryo regeneration was to ectopically express candidate gene cDNA sequences under a strong constitutive promoter. To avoid the potential risk of transcriptional transgene silencing mechanisms, which may be increased by high sequence similarity between the transgene and endogenous gene copies (Kumpatla et al. 1998), cDNA sequences of the 
maize orthologs of sorghum candidate genes were used for overexpression experiments. After identifying the maize gene with the highest sequence similarity to each sorghum candidate through a BLAST search of predicted peptide sequences, maize genes for which full-length cDNA (FLCDNA) clones were available were ordered from the Maize Full-length cDNA Project program at the University of Arizona (Soderlund et al. 2009). Table 4.2 shows the clones that were ordered, what maize genes they encode, and which sorghum candidate gene sequences they are orthologous to. The pFGC5941 vector was once again used as a backbone for building this set of constructs, where FLCDNA sequences would be inserted in the place of the ChsA intron sequence. Figure $4.5 \mathrm{C}$ displays a plasmid map representing the maize FLCDNA overexpression constructs that were assembled for this study.

Since candidate genes positively influencing somatic embryogenesis would be expected to increase the number of regenerants from samples of transformed explants, the sorghum inbred line BTx623, for which a sorghum reference genome has been assembled, and which is known to be a poorly-regenerable genotype (Do et al. 2016) was used as the transformation target. Agrobacterium-mediated transformation of overexpression constructs was performed, again following the transformation and tissue culture protocol outlined in Do et al. (2016). Almost no regeneration of embryogenic callus was observed in any treatment including the control. Out of the 376 total immature embryo explants transformed with any construct over the course of three experimental replicates, only two somatic embryos were obtained from embryogenic callus after 10- 
12 weeks on callus induction medium (much longer than the approximately 21 days required for somatic embryo induction with sorghum genotype P898012). These calli were transferred to shoot induction medium, but the regenerants did not survive to the rooting stage. Particle bombardment of overexpression constructs was also attempted on BTx623 explants following the Liu et al. (2014) protocol. However, no somatic embryos were observed among any treatment resulting from this experiment. In both the Agrobacterium-mediated transformation and particle bombardment experiments, most explants turned brown in color and did not form callus tissue but did form what appeared to be de novo root-like structures rather than somatic embryos. Explants transformed with the ZmIAA12 overexpression construct via Agrobacterium-mediated transformation appeared to regenerate a higher quantity of de novo roots than those receiving the control treatment, which is shown, along with the same construct delivered via particle bombardment in Figure 4.2. Tables 4.6 and 4.7 summarize the results of the overexpression experiments in BTx623 via Agrobacterium-mediated transformation and particle bombardment, respectively.

Inducible expression of candidate genes in sorghum lines

One final attempt at investigating the regeneration-promoting potential of sorghum candidate genes through transgenic methods was made using an inducible gene expression system. An artificial bi-directional promoter system, inducible by the steroidal compound dexamethasone (DEX), which has been previously employed in tobacco, Arabidopsis, citrus, and rice (Rossignol et al. 2014, Samalova 2021, Samalova et al. 2005, 
Wielopolska et al. 2005), was modified and commercially synthesized for use with our sorghum transformation molecular toolkit. This DEX-inducible expression cassette was cloned into the backbone of the pFGC5941 vector to yield pFGC-DEX, a map of which is displayed in Figure 4.5D. This new vector has two cloning sites flanking each end of a pOp6 promoter (derived from the lac operon) where two gene sequences may be inserted, one in reverse orientation and one in the forward.

To assess the DEX-dependent regulation of gene expression in our new construct, sequences for the visual marker genes enhanced cyan fluorescent protein (eCFP) and $\beta$-glucuronidase with an intervening intron sequence (GUS-intron) were inserted into the left and right-flanking restriction cloning sites of pFGC-DEX, respectively, to form pFGC-DEX-eCFPGUS, a map of which is shown in Figure 4.5E. An agro-infiltration experiment was then performed in leaves of Nicotiana benthamiana to assess the strength of marker gene expression when treated with DEX compared with constitutive expression of each marker gene. Fluorescence microscopy showed a clear CFP signal in leaf disks infiltrated with Agrobacterium carrying the inducible construct that had been treated with a $20 \mu \mathrm{M}$ concentration of DEX, though the signal intensity was not as high as for constitutive eCFP expression. A slight background level of CFP fluorescence was seen in leaf disks with inducible constructs that had not received the DEX treatment. The GUS staining assay was also used on leaf disks, which showed comparable levels of GUS expression between the constitutive construct and DEX-induced expression. No GUS staining was observed in leaf disks with the inducible construct that had not been 
treated with DEX. Figure 4.3 displays representative leaf disks used for the N. benthamiana marker gene expression experiments.

Since background CFP expression from the inducible plasmid was observed in the absence of DEX treatment in the agroinfiltration experiments, CFP expression was assessed this time in sorghum and maize immature embryo tissues by transient transformation. A constitutive control was once again used, and explants were incubated on tissue culture media amended with $20 \mu \mathrm{M}$ DEX (or none in the case of the negative control). Both sorghum and maize immature embryos displayed the same pattern as that seen in N. benthamiana leaf disks, where some background CFP expression was present even without DEX treatment. Figure 4.4 displays the transiently transformed samples of maize B73 embryos for each treatment under a fluorescence microscope.

Coding sequences of the sorghum orthologs of the BABY BOOM and WUSCHEL genes (SbBBM and SbWUS) were commercially synthesized along with those of the sorghum candidate genes SbIAA12, SbNAC76, SbHSCF1, SbAIL5, and SbGLP2-1. The SbWUS sequence was inserted into the left-flanking restriction cloning site of pFGC-DEX. This SbWUS-containing vector was then used as a backbone into which the six other gene coding sequences were inserted into the right-flanking cloning site to yield six distinct co-expression plasmids under the inducible control of DEX, for which a representative map is displayed in Figure 4.5F. For transformation experiments, the African sorghum landrace 'Macia' was used as it was reported in Che et al. (2018) that the maize orthologs of $B B M$ and WUS could induce regeneration in this normally non-regenerable genotype. An experiment was also performed that utilized P898012. A plasmid licensed 
from the agrobiotechnology company Corteva, PHP78891, which carries maize BBM and WUS genes and has been shown to increase regeneration frequencies in sorghum previously (Che et al. 2018), was used as a positive control for these experiments. All six coexpression plasmids, as well as the positive control plasmid and the pFGC-DEX negative control were used as treatments in the Macia transformation experiment whereas only three of the co-expression plasmids were tested in the P898012 experiment. Both of these followed their respective transformation and tissue culture protocols (Do et al. 2016, Wu et al. 2014) except for that $20 \mu \mathrm{M}$ DEX was added as an amendment to the callus induction media. In neither experiment, were any significant differences in somatic embryo count seen between the different treatments. Results of the inducible coexpression experiments in Macia and P898012 are displayed in Tables 4.8 and 4.9, respectively.

\section{Discussion}

The aim of this study was to establish a functional role for candidate genes identified from a previous RNA-seq study on the somatic embryogenesis process. Despite multiple transgenic approaches no significant effect on the rate of somatic embryo generation was found by altering the expression of any candidate gene via the transformation methods tested here. Each experimental method used had multiple drawbacks that could have occluded the observation of evidence for regulatory influence over embryogenesis. Though RNA-induced gene silencing is a reliable and efficient method for diminishing gene expression (Senthil-Kumar and Mysore 2010), partial or full functional 
redundancy of genes may prevent an observable phenotype from being expressed if only one gene is silenced at a time. It has been previously shown that in Arabidopsis, $B B M$ and other closely related AP2/ERF transcription factors such as PLT1, PLT2, AIL5, AIL6, and PLT7 have been shown through overexpression to act redundantly to increase somatic embryo formation (Horstman et al. 2017b, Tsuwamoto et al. 2010). To address this possibility with the set of candidate indole-3-acetic acid inducible (IAA) genes that were tested, co-bombardment of RNAi plasmids targeting five different RNAi sequences was used for one of the treatments in transforming Tx430 immature embryos. However, this did not lead to a significant difference in somatic embryo induction. Since so many IAA genes were differentially expressed in the RNA-seq data set, it is still possible that there are yet other genes not included among the candidates tested that can functionally compensate for the silencing of these candidate genes.

The primary issue with the experiments undertaken to test overexpression of candidate gene sequences was likely to have been the use of an untested sorghum genotype as a transformation target. Regeneration in tissue culture had been previously tested for BTx623, establishing that it was poorly regenerable using media formulations which were incorporated into the protocol for transformation of P898012 (Do et al. 2016). There had been no previous reports on transformation of this genotype. In this study, it proved to be particularly unsuitable for transformation via the protocols that were followed. However, had proper optimization experiments been undertaken, prior to performing candidate gene testing, to identify favorable tissue culture parameters for regenerating this genotype, it is possible that it might have been acceptable for gene 
functional screening. A lack of options for suitable genotypes reported in the literature at the time these experiments were performed, and the time and effort required to optimize a genotype-specific tissue culture protocol, were both factors limiting the chances of success in functional evaluation of candidate genes by overexpression.

Even if an appropriate genetic background for overexpression testing were available, the choice to utilize the maize orthologs of candidate genes may have limited the utility of the results in determining genes important for embryogenesis sorghum.

Though the rationale behind this decision was that this may help to evade epigenetic silencing of the transgene, on the other hand, the apparent maize orthologs may not confer the same function in sorghum as their cognate genes. One reason for this is that they may be unable to interact with the gene products of other endogenous sorghum genes in the same manner as the sorghum candidates themselves. Furthermore, even if a maize ortholog were to increase somatic embryo induction, this would not necessarily affirm that the corresponding sorghum candidate is directly involved in this process. Thus, in the investigation of genes controlling cellular or developmental processes, wildtype genes should be used for transgenic functional testing whenever it is feasible.

A potential problem with the DEX-inducible expression construct used in this study that was highlighted by preliminary experiments we performed was the apparently "leaky" gene transcription as shown by CFP fluorescence in the absence of DEX treatment. Since this same phenomenon was observed not only in N. benthamiana leaf disks, but also in maize and sorghum embryos, it suggests that this is not unique to a certain plant and tissue context. In Samalova et al. (2005) and Wielopolska et al. (2005) 
the pOp6/LhGR system was reported to provide stringent control over transgene expression when they were stably integrated in N. tabacum and Arabidopsis thaliana plants. One possible explanation for the discrepancy between these reported results and the ones described here could be the use of different vector backbones to carry the pOp6/LhGR cassette. An alternative explanation may be that transient expression does not provide as tight of control over DEX-dependent gene regulation as can be achieved with stable integration.

In the Agrobacterium-mediated transformation experiments for the DEX-inducible co-expression constructs, both the Macia and P898012 plants were grown outdoors under field conditions instead of in controlled greenhouse conditions as all other stock plants were. There were noticeable differences in the shape and color of the seed heads compared to those grown in the greenhouse. Also, in tissue culture the media was stained much more quickly and darkly with phenolic compounds by the tissue derived from plants grown in the field compared with typical tissue from greenhouse-grown plants. The difference in the environment where stock plants were grown may at least partially account for the lower rate of somatic embryo regeneration seen for P898012 explants in the DEX-inducible expression experiments than for explants of the same genotype in the RNAi experiments performed earlier.

Finally, the use of transient transformation methods for evaluating the control candidate genes may have over somatic embryogenesis has some inherent limits. Though transformation with developmental regulators known to promote somatic embryogenesis has given higher rates of recovery of T0 somatic embryos (Lowe et al. 2016, 
Mookkan et al. 2017), plants stably transformed with candidate gene sequences should give a much more reliable indication of whether the transgene promotes embryogenesis. Taking into account that developmental aberrations have been observed when developmental regulators which are normally expressed only at the embryonic stage are constitutively expressed (Gordon-Kamm et al. 2019, Tsuwamoto et al. 2010), using an inducible expression system to control candidate genes so that they are only transcribed at the developmental stage being tested is likely to be a more successful strategy than constitutive overexpression. However, in order to obtain a large enough quantity of immature embryo explants carrying a given stably integrated transgene to be used for testing embryogenic potential in tissue culture, it would be necessary to bring transgenic plants through at least two full generation cycles. Thus, considering the time and effort required to obtain stable transgenic stock plants for each candidate gene, this method would be infeasible for high-throughput functional screening.

\section{Materials and Methods}

\section{Plant material used}

The sorghum genotypes P898012, Tx430, BTx623, and Macia were used for the various transformation experiments in this study. Seeds of P898012, Tx430, and BTx623 were gifts from Dr. Yinghua Huang (USDA-ARS, OK) and Macia was obtained from the USDA-GRIN program in GA. The maize inbred line B73 was also used for evaluating the expression of marker genes from the dexamethasone-inducible promoter in immature 
embryos. All sorghum and maize genotypes were grown in a greenhouse at the University of Missouri, Columbia, MO under the same conditions. Greenhouse plants were grown on a $16 \mathrm{~h}$ light/ $8 \mathrm{~h}$ dark cycle at a temperature ranging from 21 to $28^{\circ} \mathrm{C}$ in 3 -galIon pots containing Promix soil media amended with Osmocote (14-14-14) (Hummert International, Earth City, MO). Plants were treated with iron at the six-leaf stage. Upon anthesis, sorghum heads were covered with a tassel bag to encourage self-pollination. Immature seeds were collected from sorghum panicles for transformation at 11-15 days following the coverage with tassel bags. For maize plants, the ends of ears were cut with a knife immediately after silking and temporarily covered with a small bag. After 1 day of growth the extended silks were pollinated with pollen collected from the tassel. The ear was then covered again. 10-11 days following pollination, immature ears were collected for transformation experiments.

Plants of the sorghum genotypes P898012 and Macia used for the dexamethasone-inducible candidate gene expression experiments were grown outdoors under field conditions at the Bradford Research Center, Columbia, MO during the summer of 2020. Seeds were planted in late June-early July and plants were watered regularly and fertilized with urea at $11 \mathrm{~g} \mathrm{~m}^{-2}$ approximately three weeks after germination. Plants were pollinated and seeds collected just as for greenhouse-grown plants described above.

Nicotiana benthamiana plants used for agro-infiltration experiments were grown from seed in small pots of Promix soil and maintained in a growth chamber at $25^{\circ} \mathrm{C}$ on a $12 \mathrm{~h}$ light/12 h dark cycle. 
Candidate gene cloning and plasmid construction

All plasmids used for transformation experiments were based on the pFGC5941 binary vector (Kerschen et al. 2004), which is compatible with both long hairpin RNAi and overexpression of an open reading frame with gene expression driven by a CaMV $35 \mathrm{~S}$ promoter and an omega enhancer element.

For RNAi constructs long hairpin RNAi sequences $300-600$ nucleotides in length matching to a segment of each gene of interest were designed using the online tool OligoWalk (Lu and Mathews 2008). Sequences were synthesized by Genscript (Piscataway, NJ) flanked by added Xbal and Ascl restriction enzyme sites on the 5' end and Swal and Avrll sites on the $3^{\prime}$ end, and inserted into the pUC57 cloning vector. This enabled the insertion in the forward orientation of the RNAi sequence for each gene into the first multiple cloning site of pFGC5941 (upstream of the ChsA intron) between the Ascl and Swal restriction sites, and an insertion of a second copy in the reverse orientation into the second multiple cloning site (downstream of the ChsA intron) using the Xbal and $A v$ rll restriction sites.

For cDNA overexpression constructs, putative maize orthologs of candidate sorghum genes were identified using the NCBI protein BLAST tool to retrieve maize genes that were best matches (with an E value cutoff of $1 \times 10^{-5}$ ) to each candidate gene. These best hits were then BLASTed back to the sorghum genome to ensure that the same sorghum gene was the top hit. cDNA clones for each maize ortholog were obtained from the Maize Full-length cDNA Project at the Arizona Genomics Institute as 
they were available (Soderlund et al. 2009). cDNA sequences were PCR-amplified from templates contained in the cloning plasmid using primers pairs with added $\mathrm{Ncol}$ (or Ascl) and BamHI (or AvrlI) sequences in the forward and reverse primers, respectively. The amplicons were then digested with the appropriate restriction enzymes and were inserted into the pFGC5941 vector backbone at the corresponding sites in place of the Chs $A$ intron sequence.

For the dexamethasone-inducible expression experiments, an inducible expression plasmid backbone was first designed by having a modified version of the bi-directional pOp6/LhGR sequence as described in Samalova et al. (2005), incorporating Tvsp and OCS $3^{\prime}$ terminators to either end of the pOp6 promoter, synthesized by Biomatik (Cambridge, Ontario, Canada). This sequence was cloned into the pFGC5941 plasmid backbone at the EcoRI and Pmel restriction sites to yield the pFGC-DEX vector. To generate a plasmid for testing expression efficiency of the pOp6 dex-inducible system, an eCFP open reading frame was PCR amplified from another plasmid ( $p F G C-e C F P$ ) and cloned into the upstream side of the pOp6 promoter in pFGC-DEX via the Ascl and Avrll restriction sites, while the GUS-intron gene sequence was amplified from the binary vector pZY102 and was cloned downstream of the pOp6 promoter via the Swal and Sall restriction sites. This yielded the plasmid pFGC-DEX-eCFPGUS, which was tested by agroinfiltration in $N$. benthamiana and transformation into sorghum and maize embryos. Coding sequences for the sorghum orthologs of BBM, WUS, and 5 candidate gene sequences were also synthesized by Biomatik. The SbWUS sequence was cloned from upstream of the pOp6 promoter via the Swal and Sall restriction sites. SbBBM and each 
candidate gene were then each inserted downstream of the pOp6 promoter of this plasmid carrying SbWUS to yield six different constructs, which were used for evaluating the effect of inducible candidate gene expression on somatic embryogenesis. SnapGene software (GSL Biotech LLC, Chicago, IL) was used to make plasmid maps.

All cloned transformation vectors were mobilized to competent cells of the Agrobacterium tumefaciens strain AGL1, which was the sole strain used for transformation.

\section{Transformation and tissue culture}

For all transformation experiments, immature sorghum seeds were removed individually from the panicles before being sterilized with a $50 \%$ bleach solution with 100 $\mu \mathrm{L}$ Tween-20 for 15 minutes under gentle shaking. Seeds were then rinsed 3 times with sterile deionized water and left under a laminar flow hood to dry for about 20 minutes. Immature embryos were then aseptically isolated from each seed using forceps and a scalpel with a \#15 blade. 20-55 embryos per treatment were placed in $1.7 \mathrm{~mL}$ microcentrifuge tubes containing liquid infection medium/osmotic medium. Once all embryos were collected for each sample, they were washed twice with fresh liquid medium to remove starchy endosperm residue.

For Agrobacterium-mediated transformation, A. tumefaciens AGL1 isolates harboring each construct to be tested in a given experiment were isolated from a glycerol stock stored at $-80^{\circ} \mathrm{C}$ on plates containing YEP medium amended with $30 \mathrm{mg} / \mathrm{L}$ rifampicin and $50 \mathrm{mg} / \mathrm{L}$ kanamycin. After $2-3$ days of incubation in the dark at $28^{\circ} \mathrm{C}$, single colonies were selected and streaked out on fresh YEP plates, which were again incubated 
for 3 days at $28^{\circ} \mathrm{C}$. At time of transformation, Agrobacterium colonies on the plates were suspended in infection medium at an O.D.550 of 0.4 in $15 \mathrm{~mL}$ Falcon tubes. Tubes were then shaken at $100 \mathrm{rpm}$ for 4-5 hours. After this time, $1 \mathrm{~mL}$ of Agrobacterium suspensions for each treatment were used to inoculate collected and washed immature embryo samples at $25^{\circ} \mathrm{C}$ for 10 minutes. The Agrobacterium suspension was then discarded and inoculated embryos were transferred to co-cultivation medium plates with the scutellum side facing upward. Plates were incubated in the dark for 3 days after which they were transferred to callus induction medium (CIM). Cultures were maintained on CIM for up to 4 weeks with subculture every two weeks. The number of somatic embryos forming on each culture plate was counted throughout this time. For transformation of the P898012 genotype, the tissue culture protocol and media formulations outlined in Do et al. (2016) were followed. For transformation of BTx623 and Macia genotypes, the tissue culture protocol and media formulations outlined in Wu et al. (2014) was followed.

For maize B73 the Agrobacterium-mediated transformation and tissue culture protocol described in Mookkan et al. (2017) was followed.

For particle bombardment transformation experiments, isolated immature embryos were directly plated scutellum-side up onto CIM. They were incubated in the dark for 7-9 days after which 10 to 12 immature embryos were placed onto the center of a petri plate containing osmotic medium over about a $2.5 \mathrm{~cm}$ radius for two hours prior to bombardment. 4 hours following bombardment, these embryos were transferred back to CIM where they were maintained for 4 weeks with subculture at two-week intervals 
as the number of somatic embryos forming from callus were recorded throughout this period. For biolistic transformation of sorghum genotypes Tx430 and BTx623, the preparation of gold particles, particle coating with DNA, bombardment, tissue culture protocol, and media formulations followed those outlined in (Liu et al. 2014).

Assessment of marker gene expression in inducible system

The expression of the GUS-intron and eCFP marker genes was first assessed by infiltrating AGL1 carrying the pFGC-DEX-eCFPGUS plasmid was infiltrated into leaves of 4 to 6 week-old N. benthamiana plants (alongside plants inoculated with two Agrobacterium isolates carrying constructs constitutively expressing each of the marker genes, respectively) using $1 \mathrm{~mL}$ needleless syringes. Two days after Agrobacterium infiltration, the same leaves were infiltrated with MES infiltration buffer either with or without dexamethasone at a $20 \mu \mathrm{M}$ concentration. 3 days later, leaf disks were taken from each leaf, which were then placed on microscope slides and examined using a Leica MZ FLIII stereo microscope equipped with a color digital camera using the CFP filter (available for use at the University of Missouri Molecular Cytology Core Facility). Following the capture of fluorescence images, leaf disks were placed in GUS staining buffer with $1 \mathrm{mg} / \mathrm{mL}$ of X-gluc and incubated at $37^{\circ} \mathrm{C}$ for 48 hours. They were then removed from the buffer and placed back on microscope slides to be photographed for GUS expression.

To evaluate the expression of marker genes in immature embryo tissue, maize B73 and sorghum P898012 embryos were transformed as described above with Agrobacterium isolates carrying pFGC-DEX-eCFPGUS, or constructs constitutively expressing 
either of the two marker genes. Media formulations were as described in Mookkan et al. (2017) or Do et al. (2016) for maize or sorghum, respectively, except that $20 \mu \mathrm{M}$ dexamethasone was added to the co-cultivation and callus induction media. Embryos were collected 10 days after transformation and analysis of marker gene expression was carried out as described above for $N$. benthamiana leaf disks.

\section{Statistical analysis}

Transformation experiments were performed in a randomized complete block design. Each experiment used 2 to 3 biological replicates. One-way ANOVA was used to compare counts of somatic embryos forming from callus between different treatments. Had any significant differences been found between treatments, Tukey's honestly significant difference procedure would have been used to determine group differences.

\section{Reference List}

Arroyo-Herrera A, Gonzalez AK, Moo RC, Quiroz-Figueroa FR, Loyola-Vargas V, Rodriguez-Zapata L, Suárez-Solís V, Castaño E. 2008. Expression of WUSCHEL in Coffea canephora causes ectopic morphogenesis and increases somatic embryogenesis. Plant Cell, Tissue and Organ Culture 94: 171-180.

Bidabadi SS, Jain SM. 2020. Cellular, molecular, and physiological aspects of in vitro plant regeneration. Plants 9: 702.

Che P, Anand A, Wu E, Sander JD, Simon MK, Zhu W, Sigmund AL, Zastrow-Hayes G, Miller M, Liu D. 2018. Developing a flexible, high-efficiency Agrobacteriummediated sorghum transformation system with broad application. Plant biotechnology journal 16: 1388-1395. 
Deng W, Luo K, Li Z, Yang Y. 2009. A novel method for induction of plant regeneration via somatic embryogenesis. Plant Science 177: 43-48.

Do PT, Lee H, Mookkan M, Folk WR, Zhang ZJ. 2016. Rapid and efficient Agrobacteriummediated transformation of sorghum (Sorghum bicolor) employing standard binary vectors and bar gene as a selectable marker. Plant cell reports 35: 20652076.

Elhiti M, Stasolla C, Wang A. 2013. Molecular regulation of plant somatic embryogenesis. In Vitro Cellular \& Developmental Biology-Plant 49: 631-642.

Elhiti M, Tahir M, Gulden RH, Khamiss K, Stasolla C. 2010. Modulation of embryoforming capacity in culture through the expression of Brassica genes involved in the regulation of the shoot apical meristem. Journal of experimental botany 61 : 4069-4085.

Gazzarrini S, Tsuchiya Y, Lumba S, Okamoto M, McCourt P. 2004. The transcription factor FUSCA3 controls developmental timing in Arabidopsis through the hormones gibberellin and abscisic acid. Developmental cell 7: 373-385.

Gordon-Kamm B, Sardesai N, Arling M, Lowe K, Hoerster G, Betts S, Jones T. 2019. Using morphogenic genes to improve recovery and regeneration of transgenic plants. Plants 8: 38.

Harding EW, Tang W, Nichols KW, Fernandez DE, Perry SE. 2003. Expression and maintenance of embryogenic potential is enhanced through constitutive expression of AGAMOUS-Like 15. Plant Physiology 133: 653-663.

Hecht V, Vielle-Calzada J-P, Hartog MV, Schmidt ED, Boutilier K, Grossniklaus U, de Vries SC. 2001. The Arabidopsis SOMATIC EMBRYOGENESIS RECEPTOR KINASE 1 gene is expressed in developing ovules and embryos and enhances embryogenic competence in culture. Plant Physiology 127: 803-816.

Horstman A, Bemer M, Boutilier K. 2017a. A transcriptional view on somatic embryogenesis. Regeneration 4: 201-216.

Horstman A, Li M, Heidmann I, Weemen M, Chen B, Muino JM, Angenent GC, Boutilier K. 2017b. The BABY BOOM transcription factor activates the LEC1-ABI3-FUS3LEC2 network to induce somatic embryogenesis. Plant physiology $175: 848-857$. 
Huang S, Hill RD, Wally OS, Dionisio G, Ayele BT, Jami SK, Stasolla C. 2014. Hemoglobin control of cell survival/death decision regulates in vitro plant embryogenesis. Plant physiology 165: 810-825.

Kerschen A, Napoli CA, Jorgensen RA, Müller AE. 2004. Effectiveness of RNA interference in transgenic plants. FEBS letters 566: 223-228.

Kumpatla SP, Chandrasekharan MB, Iyer LM, Guofu L, Hall TC. 1998. Genome intruder scanning and modulation systems and transgene silencing. Trends in Plant Science 3: 97-104.

Liu G, Campbell BC, Godwin ID. 2014. Sorghum genetic transformation by particle bombardment. Pages 219-234. Cereal genomics, Springer.

Lotan T, Ohto M-a, Yee KM, West MA, Lo R, Kwong RW, Yamagishi K, Fischer RL, Goldberg RB, Harada JJ. 1998. Arabidopsis LEAFY COTYLEDON1 is sufficient to induce embryo development in vegetative cells. Cell 93: 1195-1205.

Lowe K, La Rota M, Hoerster G, Hastings C, Wang N, Chamberlin M, Wu E, Jones T, Gordon-Kamm W. 2018. Rapid genotype “independent” Zea mays L. (maize) transformation via direct somatic embryogenesis. In Vitro Cellular \& Developmental Biology-Plant 54: 240-252.

Lowe K, Wu E, Wang N, Hoerster G, Hastings C, Cho M-J, Scelonge C, Lenderts B, Chamberlin M, Cushatt J. 2016. Morphogenic regulators Baby boom and Wuschel improve monocot transformation. The Plant Cell 28: 1998-2015.

Loyola-Vargas VM, De-la-Peña C, Galaz-Avalos R, Quiroz-Figueroa FR. 2008. Plant tissue culture. Pages 875-904. Molecular Biomethods Handbook, Springer.

Lu ZJ, Mathews DH. 2008. Efficient siRNA selection using hybridization thermodynamics. Nucleic acids research 36: 640-647.

Maher MF, Nasti RA, Vollbrecht M, Starker CG, Clark MD, Voytas DF. 2020. Plant gene editing through de novo induction of meristems. Nature biotechnology 38: 8489.

Matthew L. 2004. RNAi for plant functional genomics. Comparative and Functional Genomics 5: 240-244. 
Mookkan M, Nelson-Vasilchik K, Hague J, Zhang ZJ, Kausch AP. 2017. Selectable marker independent transformation of recalcitrant maize inbred $B 73$ and sorghum P898012 mediated by morphogenic regulators BABY BOOM and WUSCHEL2. Plant cell reports 36: 1477-1491.

Mursyanti E, Purwantoro A, Moeljopawiro S, Semiarti E. 2015. Induction of somatic embryogenesis through overexpression of ATRKD4 genes in Phalaenopsis "Sogo Vivien". Indonesian Journal of Biotechnology 20: 42-53.

Rossignol P, Orbović V, Irish VF. 2014. A dexamethasone-inducible gene expression system is active in Citrus plants. Scientia Horticulturae 172: 47-53.

Samalova M. 2021. The steroid-inducible pOp6/LhGR gene expression system is fast, sensitive and does NOT cause plant growth defects in rice (Oryza sativa). bioRxiv.

Samalova M, Brzobohaty B, Moore I. 2005. pOp6/LhGR: a stringently regulated and highly responsive dexamethasone-inducible gene expression system for tobacco. The Plant Journal 41: 919-935.

Senthil-Kumar M, Mysore KS. 2010. RNAi in plants: recent developments and applications in agriculture. Gene silencing: theory, techniques and applications: 183-199.

Small I. 2007. RNAi for revealing and engineering plant gene functions. Current Opinion in Biotechnology 18: 148-153.

Smith RH. 2012. Plant tissue culture: techniques and experiments: Academic Press.

Soderlund C, Descour A, Kudrna D, Bomhoff M, Boyd L, Currie J, Angelova A, Collura K, Wissotski M, Ashley E. 2009. Sequencing, mapping, and analysis of 27,455 maize full-length cDNAs. PLoS Genet 5: e1000740.

Thakare D, Tang W, Hill K, Perry SE. 2008. The MADS-domain transcriptional regulator AGAMOUS-LIKE15 promotes somatic embryo development in Arabidopsis and soybean. Plant physiology 146: 1663-1672.

Tsuwamoto R, Yokoi S, Takahata Y. 2010. Arabidopsis EMBRYOMAKER encoding an AP2 domain transcription factor plays a key role in developmental change from vegetative to embryonic phase. Plant molecular biology 73: 481-492. 
Wang X, Niu Q-W, Teng C, Li C, Mu J, Chua N-H, Zuo J. 2009. Overexpression of PGA37/MYB118 and MYB115 promotes vegetative-to-embryonic transition in Arabidopsis. Cell research 19: 224-235.

Wielopolska A, Townley H, Moore I, Waterhouse P, Helliwell C. 2005. A high-throughput inducible RNAi vector for plants. Plant Biotechnology Journal 3: 583-590.

Wójcikowska B, Jaskóła K, Gąsiorek P, Meus M, Nowak K, Gaj MD. 2013. LEAFY COTYLEDON2 (LEC2) promotes embryogenic induction in somatic tissues of Arabidopsis, via YUCCA-mediated auxin biosynthesis. Planta 238: 425-440.

Wu E, Lenderts B, Glassman K, Berezowska-Kaniewska M, Christensen H, Asmus T, Zhen S, Chu U, Cho M-J, Zhao Z-Y. 2014. Optimized Agrobacterium-mediated sorghum transformation protocol and molecular data of transgenic sorghum plants. In Vitro Cellular \& Developmental Biology-Plant 50: 9-18.

Yin K, Gao C, Qiu J-L. 2017. Progress and prospects in plant genome editing. Nature plants 3: 1-6.

Zhu S-p, Wang J, Ye J-I, Zhu A-D, Guo W-w, Deng X-x. 2014. Isolation and characterization of LEAFY COTYLEDON 1-LIKE gene related to embryogenic competence in Citrus sinensis. Plant Cell, Tissue and Organ Culture (PCTOC) 119: 1-13. 

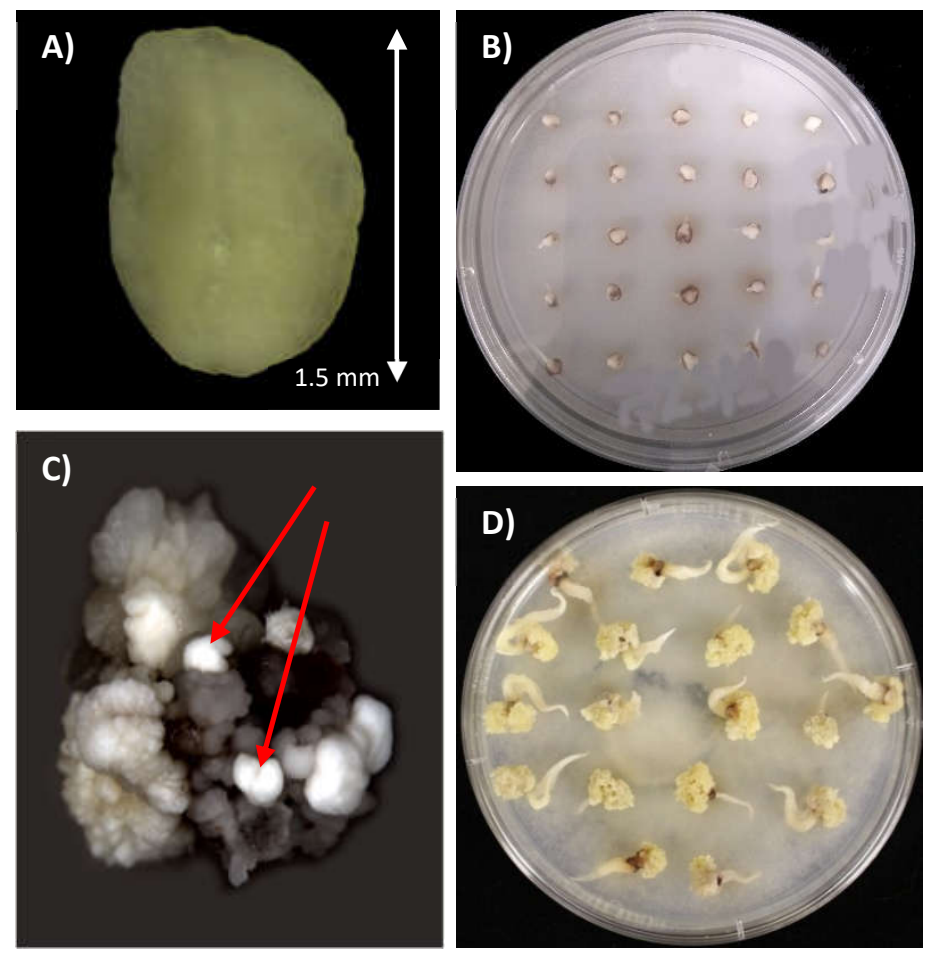

Figure 4.1: The process of somatic embryogenesis in sorghum. A) Immature sorghum zygotic embryo explants are extracted from the seed under sterile conditions 11-15 days after pollination when at an ideal size of 1.2-1.5 $\mathrm{mm}$ in length. At this point, explants may be inoculated with Agrobacterium for the purpose of transformation and placed into co-cultivation conditions for three days. They are then transferred onto an agar plate containing callus induction medium (CIM) as in B), where plant growth regulators and other components of the medium induce the explants to form callus tissue. After approximately 2 weeks on CIM, somatic embryos may become visible on the surface of calli as shown by the red arrows in C). A plate full of calli (with extended radicles from the zygotic embryo) is shown in D). Panel D) was adapted from figure 2 in Do et al. (2016). 


\section{Particle bombardment}
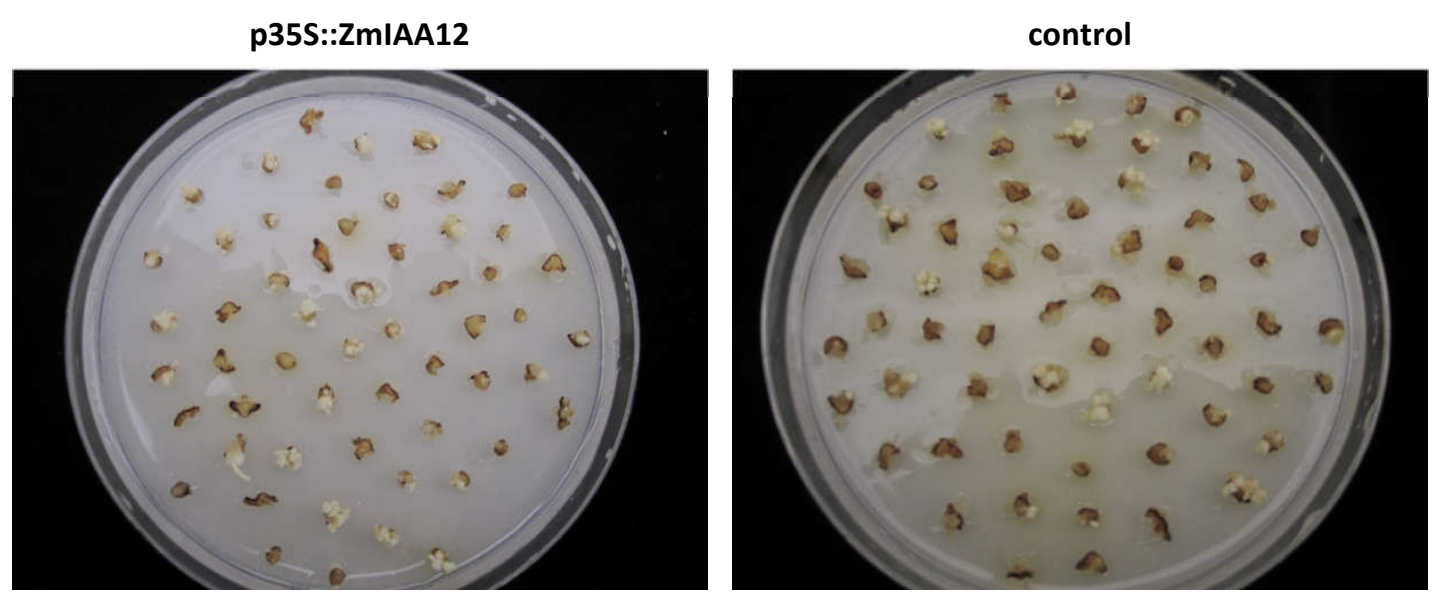

\section{Agrobacterium-mediated transformation}
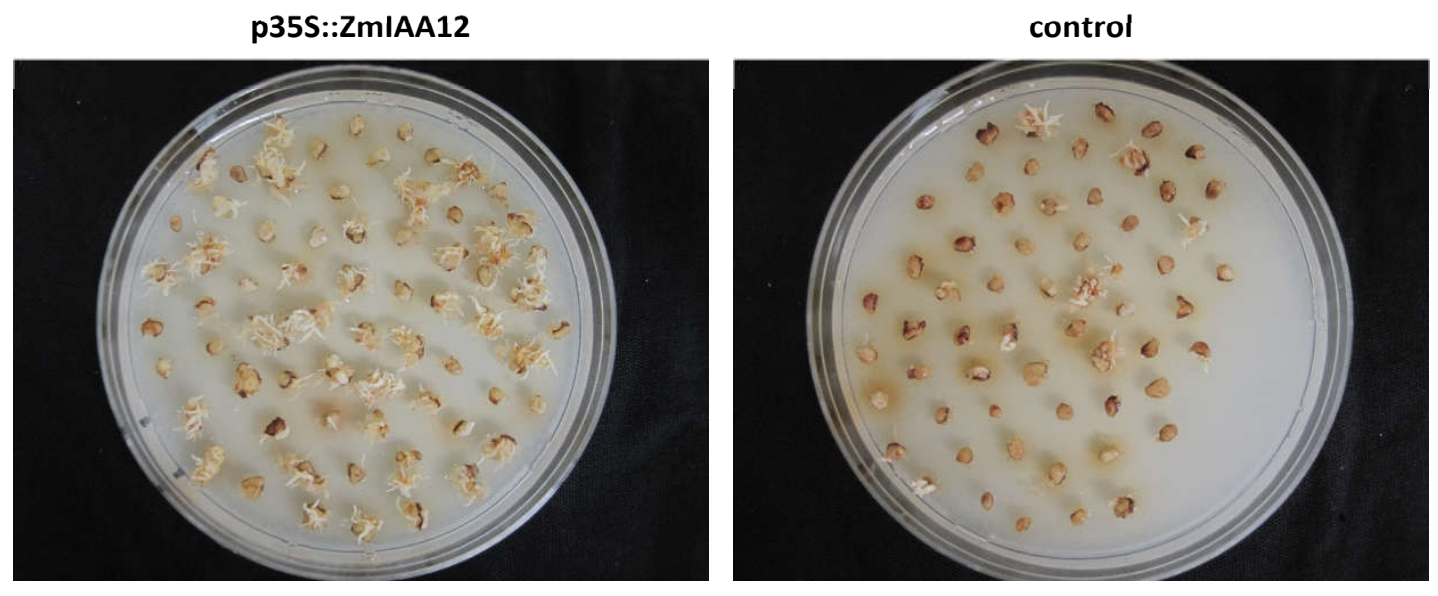

Figure 4.2: Overall effect of particle bombardment and Agrobacterium-mediated

transformation on sorghum genotype BTx623. Experiments in which BTx623 embryos were either transformed with the full-length cDNA of ZmIAA12 under the CaMV 35S promoter or received the negative control treatment (bombardment with uncoated microparticles for gene gun and inoculation with an AGL1 isolate lacking a binary plasmid for Agrobacterium-mediated transformation). Nearly all explants failed to form embryogenic callus on CIM. Instead, many directly formed de novo root-like structures as observed 3 weeks following either Agrobacterium infection or particle bombardment. 
When the p35S::ZmIAA12 construct was delivered by Agrobacterium-mediated transformation, explants produced noticeably more de novo root structures than in the control treatment. 


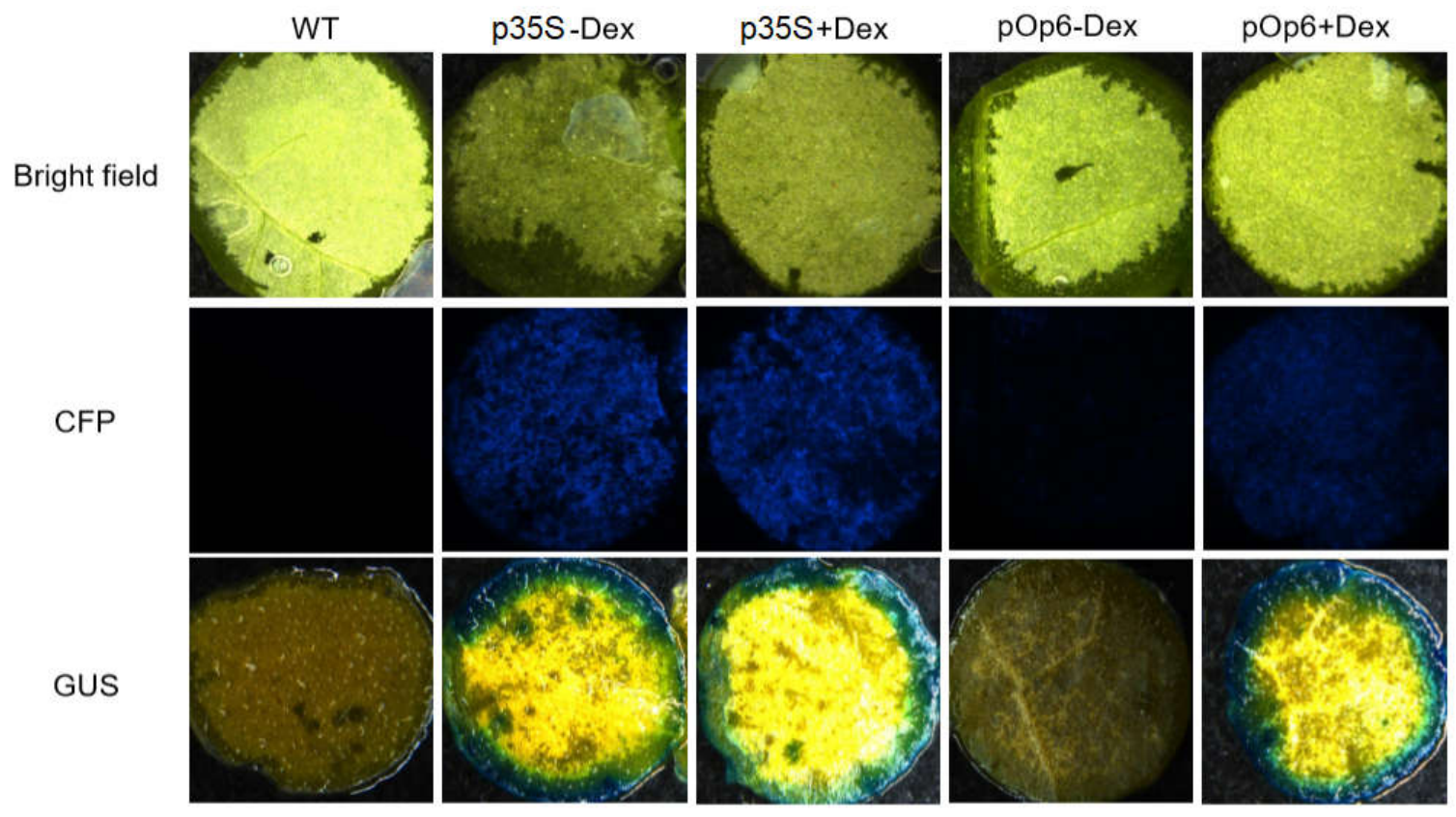

Figure 4.3: Representative $N$. benthamiana leaf disk samples for testing of marker gene expression in the bi-directional dexamethasone-inducible construct (pFGC-DEXeCFPGUS). p35S::eCFP and p35S::GUS-intron constructs were used as positive controls for the CFP fluorescence and GUS staining experiments, respectively (denoted as p35S). Leaves infiltrated with Agrobacterium isolates carrying the inducible construct (denoted as pOp6) or constitutive control constructs were either treated with dexamethasone (DEX) at a concentration of $20 \mu \mathrm{M}(+\mathrm{Dex})$ or received a mock treatment (-Dex). For the fluorescence testing, DEX-induced CFP intensity was higher than that in the mock treatment but did not reach the level of fluorescence conferred by constitutive expression of eCFP. There was also some visually detectable level of CFP fluorescence in the mocktreated leaf disks carrying the inducible construct, indicating that DEX induction may not provide a tight control over gene expression within the context of the pFGC-DEX vector. For the GUS expression test, DEX-dependent GUS staining was comparable to that seen 
with constitutive GUS expression, whereas no background GUS staining was visually apparent in the mock treated leaf disks. 

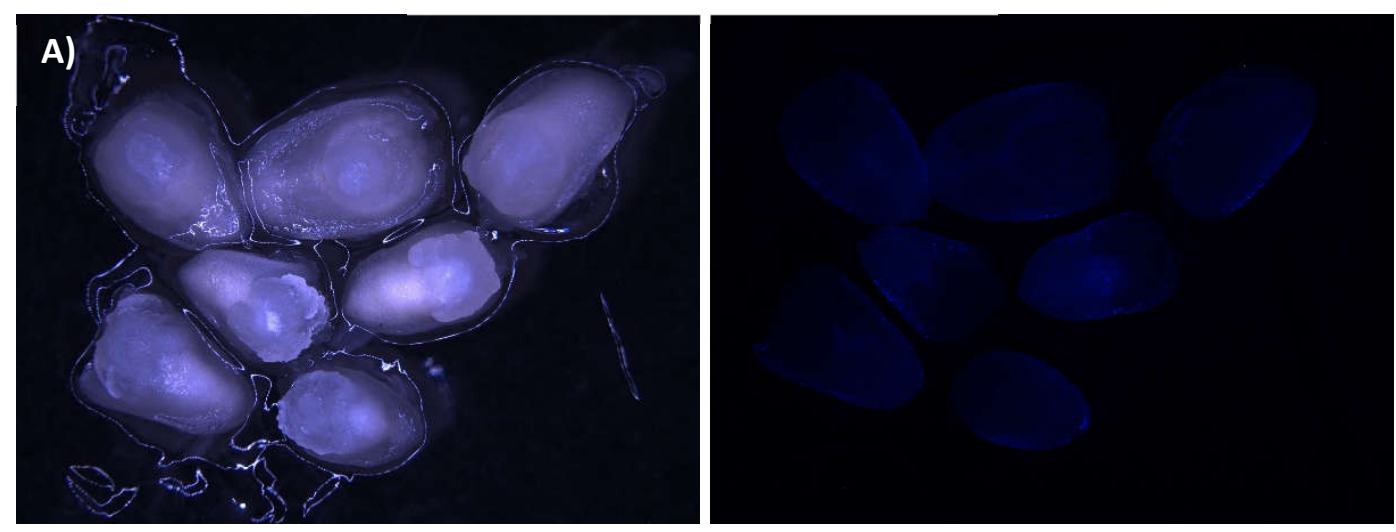

\section{pOp6::eCFP + $20 \mu M$ DEX}
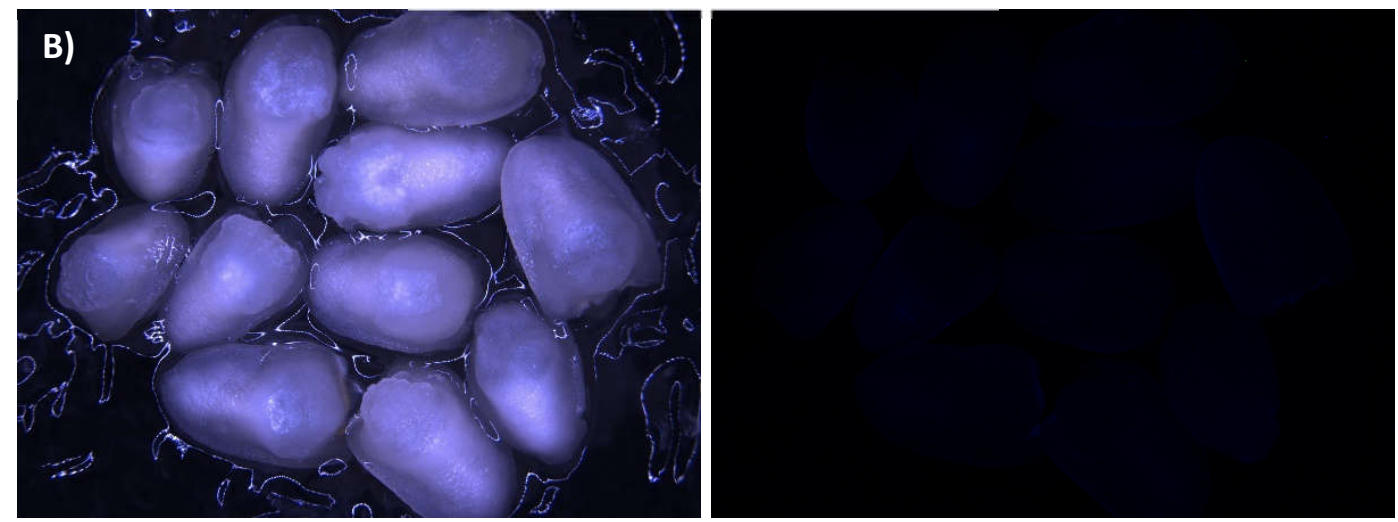

pOp6::eCFP (no DEX ctrl)
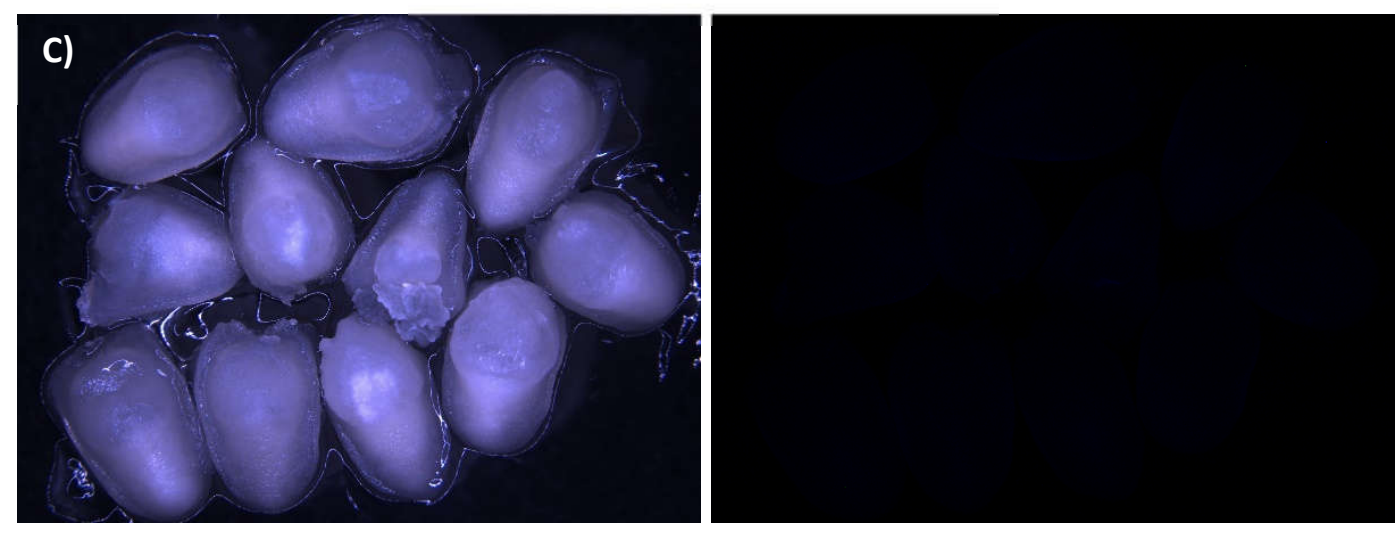

Figure 4.4: Test of DEX-inducible CFP expression in maize B73 immature embryos

transformed with the construct containing the p0p6 promoter. Bright field images of

embryos are shown on the left panels, while the right panels show fluorescence images 
under a CFP filter. As observed previously with CFP fluorescence testing by agro-infiltration in N. benthamiana, there is apparently some background eCFP expression under the pOp6 promoter even with mock treatment. A) constitutive CFP expression under the CaMV 35S promoter. B) DEX treatment-induced expression under the pOp6 promoter. C) CFP expression under the pOp6 promoter receiving mock treatment. 

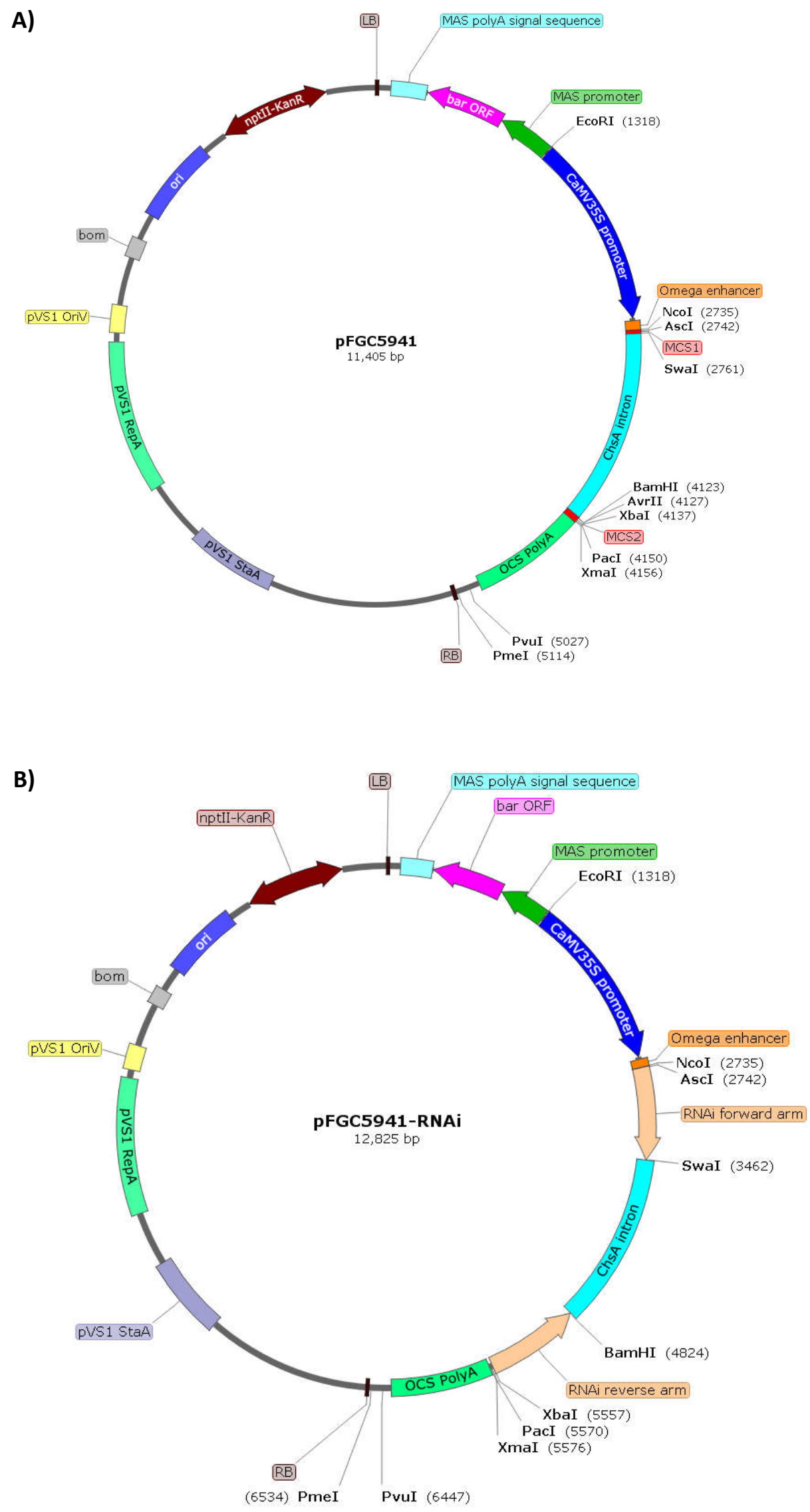

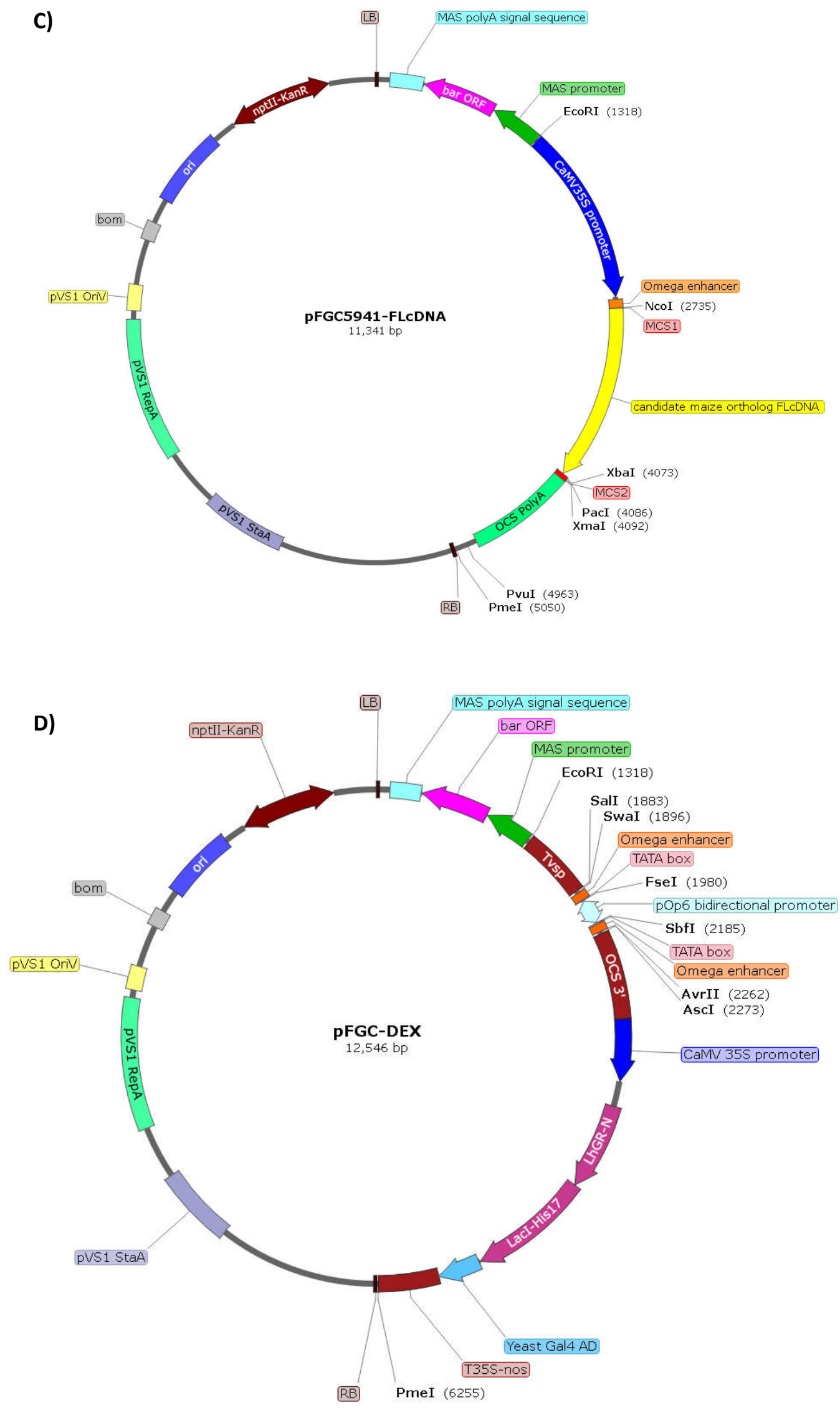

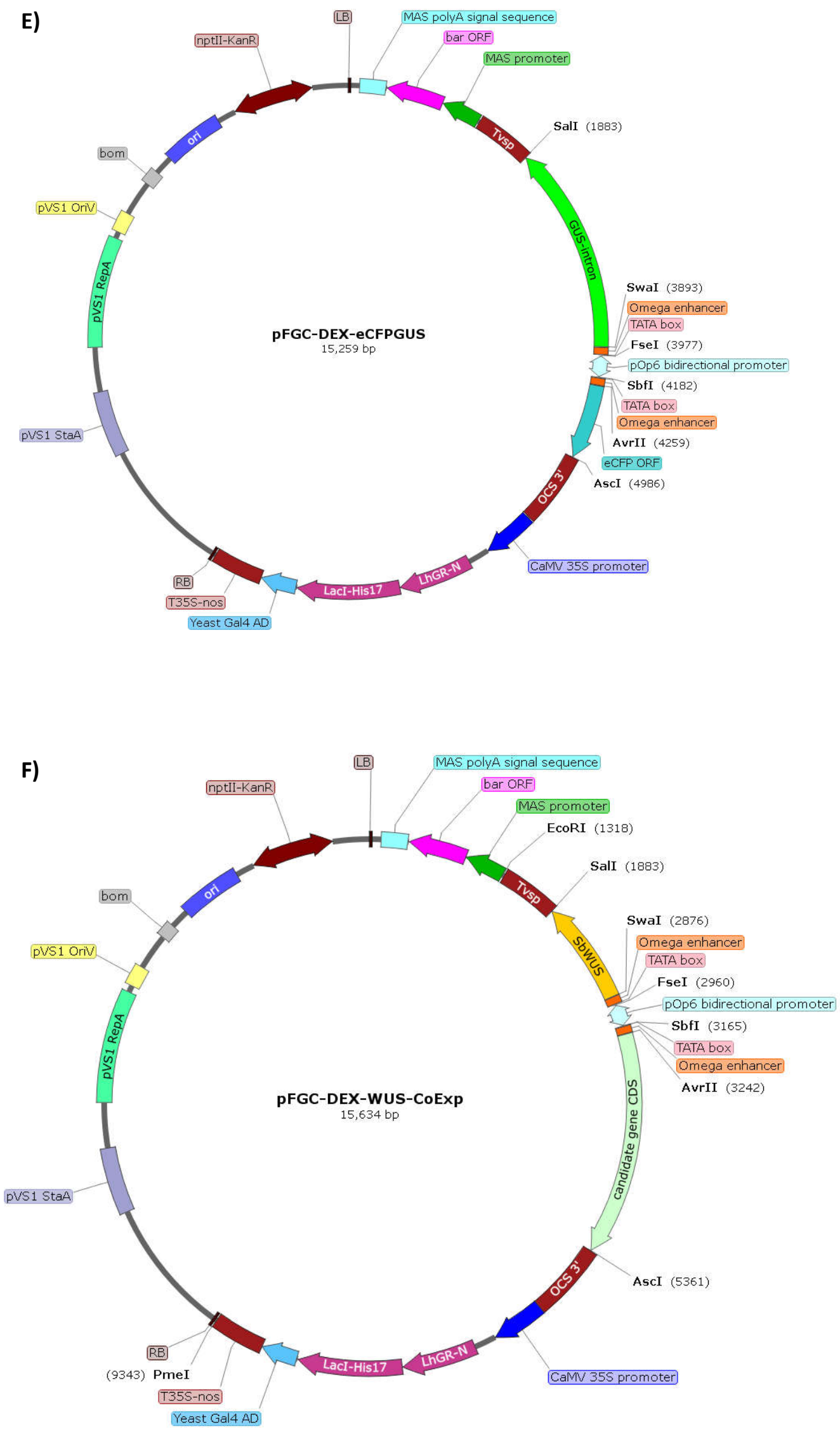
Figure 4.5: Representative and composite plasmids used in this study displaying essential sequences and restriction sites. A) The pFGC5941 binary vector on which all expression constructs designed for use in this study were based. B) A composite plasmid displaying the insertion of forward and reverse arms of long hairpin RNAi sequences inserted into the pFGC5941 backbone on either flank of the ChsA intron sequence. When the resulting transgene cassette is expressed in planta, it forms a complementary double-stranded RNA structure containing short RNA sequences that can serve as a substrate for the plant's endogenous RNAi silencing system to knock down expression of individual target genes. 10 such plasmids were cloned to each target an individual candidate gene for silencing. C) A composite plasmid showing the insertion of a maize fulllength cDNA sequence in place of the ChsA intron in the pFGC5941 backbone. The resulting transgene would lead to constitutive overexpression of the gene of interest in planta. 10 such plasmids were cloned for the purpose of overexpression testing. D) A bidirectional, DEX-inducible cassette, based on a modified version of that reported in Samalova et al. (2005), which was constructed by DNA synthesis was cloned into the pFGC5941 backbone at the EcoRI and Pmel restriction sites to yield the new inducible expression vector pFGC-DEX. E) A GUS sequence containing a plant-active intron and an eCFP coding sequence were inserted into the cloning sites at the right and left flanks, respectively, of the pOp6 promoter in pFGC-DEX to yield pFGC-DEX-eCFPGUS, which was used in experiments testing the inducible expression. F) A synthesized coding sequence for the SbWUS gene was inserted into the cloning site at the left flank of the pOp6 promoter in pFGC-DEX. This intermediate plasmid was then used as a vector backbone for 
cloning of several constructs used for testing the effect of developmental gene co-expression on somatic embryogenesis. These constructs resulted from the insertion of various sorghum gene coding sequences into the cloning site to the right flank of pOp6, which included SbBBM (the pOp6::SbWUS;SbBBM plasmid was used as a positive control for these experiments) as well as SbIAA12, SbNAC76, SbHSCF1, SbAIL5, and SbGLP2-1. The map displayed in panel $\mathbf{F}$ is a composite representing all of these co-expression plasmids. 
AGGACCGGATCGGACAATATGTTGTCATGACCTTCTT-

GTTTTTTTTTTCGTTTTCATTATTTTTGTTCTTCTGGTTGGATCCCCAAGCCGAAGGATGAGCTT GGTGTTTCGAAGCGCATACACGGATGTGTGCGGCTTTGATTCTGTAAGAC-

GATTAGGTCTTTTTAGCAGCACAAAGGAGATGCATGCTGAGGTTGTTTCAGTTTACTCTCCATGT GTTTGTACAAACATTTAAATGCCTAGGGC-3'

\title{
Swal Avrll
}

ARF5-like/ARF20/ARF19 | Sobic.004G037800.1 RNAi target sequence (722 bp)

\author{
Pacl Ascl \\ 5' -CCTTAATTAACGGCGCGCCGCGAATAGGCTGGAAGTTAGTCTACAAGGATCATGAG-
} GATGACATCCTACTTCTCGGTGATGACCCATGGGAGGAGTTTGTGAATTGTGTGAAGTGCATTAG GATCCTATCCCCCCAAGAAGTGCAACAGATGAGCTTAGACGGTGATTTGGG-

GAACAATGTCCTCTCCAACCAGGCTTGCAGCAGCTCA GATGGTGGGAATGCCTGGAAGCCTCGCT GTGACCAGAACCCTGGTAACCCTTCGATCGGCTTCTACGATCAATTTGAATGAC-

GTGATCACAGAATGGGAGATGAGAACTATGGTTGCAAAAAGCAACCAGTGTCCAGCCATCTACAG AATTATCGCCAAGCAATGCAGGATGCTGTAGACGTCTAGGCTTTTATAGCTGGCAGAGCACTCCTCAGATTAGGGTATGTTTTAGATATATAGAGCCATTGCAGAGTCAAAACTTCAGACAGAGGG TTAGGCAAAGTCTGAACTACAGACA GAGTAGGCAGATCTGAAGATAAAGTATTTTGATGGAGAGTATTTGTAATTGTGTATTGAACTGCTGCTGGGGTTGCTTGAAACCCATGTAGCACTATGAGT CTCAGTTCAGAAAATACTGAAGCAGATTTAAGAGGTTCTACTGATTAGTACTATTTGTGTAATGTGAATGGCCTTTAGGTGTTCTTACACATGCTTGATTTAAATGCCTAGGGC-3'

\section{Avrll}

\section{Swal}

Heat-shock complementing factor 1/AP2-like ethylene response protein 5 | Sobic.010G215100.2 RNAi target sequence (360 bp)

Xbal Ascl
5' -CCTCTAGAGGCGCGCACATTCCTTATGCTGCTGCTGCTGCTGCTATGGTTTCTG-
GATCAGCTGGCTACGAGGGCTCCACCGGAAACAATGGAACTGGGTTACCAGTAGCACTAGCACC
AGCACGGCTCCCCAGTACTACAACTATCTGTTCGGGATGGAGTAGGTGCACTGACAA-
GCTAGCTAGCTGAAATCAGGTGATAGGCCTAGGAAGCTAGGACTGACATTGAACTGATCACAATT
TTGGCTGCTACATATGCCCATGTATGGGTATGGCTGGGGTGCATCCTTCTGCTATTCATGA-
TATTTCCTGACTTACTGTAGCAGAGAGCTCATTTACCATCATTTAAATGGGATCCGC -3'

\section{Swal BamHI}

transcription factor bHLH150 | Sobic.010G212800.1 RNAi target sequence (451 bp)

Xbal Ascl
5' -CCTCTAGAGGCGCGCATGAAACAAGGTCATGGGCGGCGATCTGACGGCGATTAGAGAG-
GAC-
GGATCTCCGAGCTCGTGGTAGACGTAATGGTGAAGAACTGATCAGGGTTTTGTCTCGCTTCATTA
GTGGGACATGTGTAAGCTAGCTAGACACAGCTACTGTTGTACGGCGACTCGGGGAG-
GAAAGGGACAACATGCTATTGTAGATGAATATAGAAATGGGTGCCAATTAGTCTCACTTATGGC
CACTCATTGCCAACACAGAAAGAGGGGAAAAGGAGTGAAAATACCAAAAAGATCATT-
GATCTGCTAGCTATATTTGCTCCCTCCATGCAAGCTACTTTTCTCTGACGAGGACAACGGTACCT
CTACAAGCATCTATGTAAACTCCCATCCAACATGGCGCGTTGGGGAA-
GCCCTTAAAAATGCTAATTAAATGGGATCCGC-3'

Swal BamHI 
Figure 4.6: RNAi sequences ordered for DNA synthesis targeting 10 candidate genes.

The inner restriction enzyme sites colored and underlined in red were those used to insert each sequence into the pFGC5941 backbone in the forward orientation at the multiple cloning site to the left flank of the ChsA intron. The resulting intermediate plasmids for each RNAi segment were then used as backbones for the insertion of the same sequence, this time in the reverse orientation, at the multiple cloning site to the right flank of the ChsA intron via the outer restriction enzyme sites colored and underlined in green. 
Table 4.1: Results of Agrobacterium-mediated transformation RNAi experiment 1 in sorghum P898012

\begin{tabular}{lrrr}
\hline & $\begin{array}{c}\text { \# immature zy- } \\
\text { gotic embryos } \\
\text { transformed }\end{array}$ & $\begin{array}{c}\text { \# embryogenic } \\
\text { calli formed }\end{array}$ & \multicolumn{2}{c}{$\begin{array}{c}\text { \# somatic embryos } \\
\text { counted }\end{array}$} \\
\hline control & 99 & 42 & 189 \\
p35S::Sbnac76-L.RNAi & 118 & 50 & 194 \\
p35S::Sbgata23-L.RNAi & 140 & 49 & 132 \\
p35S::Sbhscf1.RNAi & 108 & 55 & 121 \\
p35S::Sbbhlh150.RNAi & 117 & 39 & 148 \\
\hline
\end{tabular}

*Represents the total number of immature zygotic embryos used for three experimental replicates. 30-40 embryos were used per replicate. 
Table 4.2: Maize full-length cDNA clones obtained that are orthologous to candidate sorghum genes

\begin{tabular}{|c|c|c|c|c|c|c|}
\hline $\begin{array}{l}\text { ID of maize } \\
\text { gene }\end{array}$ & $\begin{array}{l}\text { ID of orthologous } \\
\text { sorghum candi- } \\
\text { date gene (name) }\end{array}$ & $\begin{array}{l}\text { Protein } \\
\text { percent } \\
\text { identity }\end{array}$ & E-value & $\begin{array}{l}\text { FL-cDNA clone } \\
\text { accession }\end{array}$ & $\begin{array}{l}\text { NCBI accession } \\
\text { of clone }\end{array}$ & $\begin{array}{l}\text { Success- } \\
\text { fully } \\
\text { cloned into } \\
\text { pFGC5941 } \\
\text { backbone? }\end{array}$ \\
\hline LOC100193444 & $\begin{array}{l}\text { Sobic.003G044900 } \\
\text { (SbIAA1) }\end{array}$ & $90.13 \%$ & $8.0 \mathrm{E}-122$ & ZM_BFb0078M19 & B_BT035709.1 & Yes \\
\hline LOC103629850 & $\begin{array}{l}\text { Sobic.004G112200 } \\
\text { (NAC076-like) }\end{array}$ & $85.71 \%$ & 0.0 & ZM_BFb0329L19 & B_BT038816.1 & No \\
\hline LOC100194253 & $\begin{array}{l}\text { Sobic.009G065000 } \\
\text { (SbIAA15) }\end{array}$ & $58.33 \%$ & $5.0 \mathrm{E}-33$ & ZM_BFc0170D01 & C_BT034414.1 & Yes \\
\hline LOC100282670 & $\begin{array}{l}\text { Sobic.010G052700 } \\
\text { (SbIAA20) }\end{array}$ & $73.68 \%$ & $4.0 \mathrm{E}-88$ & ZM_BFb0101E15 & B_BT068319.1 & Yes \\
\hline LOC103638861 & $\begin{array}{l}\text { Sobic.010G215100 } \\
\text { (SbHSCF1) }\end{array}$ & $87.40 \%$ & 0.0 & ZM_BFc0057019 & C_BT040003.1 & Yes \\
\hline LOC100216870 & $\begin{array}{l}\text { Sobic.001G161500 } \\
\text { (SbIAA12) }\end{array}$ & $77.16 \%$ & 2.0E-96 & ZM_BFb0178M05 & B_BT037481.1 & Yes \\
\hline LOC100193780 & $\begin{array}{l}\text { Sobic.001G164800 } \\
\text { (SbB3bdp) }\end{array}$ & $77.49 \%$ & 0.0 & ZM_BFb0097001 & B_BT036138.1 & No \\
\hline LOC100384122 & $\begin{array}{l}\text { Sobic.006G245500 } \\
\text { (SbAlL5) }\end{array}$ & $81.55 \%$ & 0.0 & ZM_BFb0341N12 & B_BT069215.1 & No \\
\hline LOC100280600 & $\begin{array}{l}\text { Sobic.002G055900 } \\
\text { (SbIAA24) }\end{array}$ & $84.88 \%$ & 4.00E-99 & ZM_BFb0225J03 & B_BT068479.2 & Yes \\
\hline LOC100502435 & $\begin{array}{l}\text { Sobic.004G233200 } \\
\text { (SbDRFL2a) }\end{array}$ & $80.12 \%$ & $5.0 \mathrm{E}-154$ & ZM_BFc0039L03 & C_BT088163.1 & Yes \\
\hline LOC541815 & $\begin{array}{l}\text { Sobic.001G449600 } \\
\text { (SbHB1) }\end{array}$ & $94.55 \%$ & $1.0 \mathrm{E}-114$ & ZM_BFc0066D21 & C_BT040115.1 & Yes \\
\hline LOC103654141 & $\begin{array}{l}\text { Sobic.004G338500 } \\
\text { (SbJMJ16) }\end{array}$ & $87.26 \%$ & 0.0 & ZM_BFb0306B04 & B_BT062499.1 & No \\
\hline LOC100272363 & $\begin{array}{l}\text { Sobic.006G118400 } \\
\text { (SbGA20ox2) }\end{array}$ & $92.67 \%$ & 0.0 & ZM_BFb0287B01 & B_BT038561.1 & Yes \\
\hline LOC100193182 & $\begin{array}{l}\text { Sobic.010G031900 } \\
\text { (SbENOD93) }\end{array}$ & $96.52 \%$ & $6.0 \mathrm{E}-79$ & ZM_BFb0058H02 & B_BT035380.1 & Yes \\
\hline
\end{tabular}


Table 4.3: Results of Agrobacterium-mediated transformation RNAi experiment 2 in sorghum P898012

\begin{tabular}{|c|c|c|c|}
\hline cassette delivered & $\begin{array}{l}\text { \# immature zygotic } \\
\text { embryos trans- } \\
\text { formed* }\end{array}$ & $\begin{array}{l}\text { \# embryogenic calli } \\
\text { formed }\end{array}$ & $\begin{array}{c}\text { \# somatic embryos } \\
\text { counted }\end{array}$ \\
\hline control & 92 & 24 & 153 \\
\hline p35S::Sbiaa15.RNAi & 90 & 34 & 140 \\
\hline p35S::Sbiaa9.RNAi & 94 & 27 & 156 \\
\hline p35S::Sbiaa12.RNAi & 90 & 28 & 147 \\
\hline
\end{tabular}

*Represents the total number of immature zygotic embryos used for three experimental replicates. 30-40 embryos were used per replicate.

Table 4.4: Results of Agrobacterium-mediated transformation RNAi experiment 3 in sorghum P898012

\begin{tabular}{lrrr}
\hline & $\begin{array}{c}\text { \# immature zy- } \\
\text { gotic embryos } \\
\text { transformed* }\end{array}$ & $\begin{array}{c}\text { \# embryogenic calli } \\
\text { formed }\end{array}$ & \multicolumn{2}{c}{$\begin{array}{c}\text { \# somatic embryos } \\
\text { counted }\end{array}$} \\
\hline control & 108 & 47 & 139 \\
p35S::Sbiaal.RNAi & 97 & 33 & 170 \\
p35S::Sbiaa20.RNAi & 98 & 35 & 94 \\
p35S::Sbarf5-L.RNAi & 100 & 40 & 116 \\
\hline
\end{tabular}

*Represents the total number of immature zygotic embryos used for three experimental replicates. 30-40 embryos were used per replicate.

Table 4.5: Results of particle bombardment RNAi experiment in sorghum Tx430

\begin{tabular}{lccr}
\hline & $\begin{array}{c}\text { \# immature zygotic } \\
\text { embryos trans- } \\
\text { cormed* }\end{array}$ & $\begin{array}{c}\text { \# embryogenic calli } \\
\text { formed }\end{array}$ & \multicolumn{2}{c}{$\begin{array}{c}\text { \# somatic embryos } \\
\text { counted }\end{array}$} \\
\hline control & 49 & 40 & 283 \\
p35S::Sbiaa1.RNAi & 48 & 37 & 194 \\
p35S::Sbiaa9.RNAi & 48 & 29 & 240 \\
p35S::Sbiaa12.RNAi & 51 & 42 & 222 \\
p35S::Sbiaa15.RNAi & 48 & 31 & 319 \\
p35S::Sbiaa20.RNAi & 50 & 33 & 365 \\
All iaa.RNAi con- & 49 & 28 & 206 \\
structs co-bom- & & & \\
barded & 49 & &
\end{tabular}


Table 4.6: Results of Agrobacterium-mediated transformation overexpression experiment in sorghum BTx623

\begin{tabular}{lcccc}
\hline & $\begin{array}{c}\text { \# immature zygotic } \\
\text { embryos trans- } \\
\text { formed* }\end{array}$ & $\begin{array}{c}\text { \# embryogenic calli } \\
\text { cassette delivered }\end{array}$ & 78 & \multicolumn{2}{c}{$\begin{array}{c}\text { \# somatic embryos } \\
\text { counted }\end{array}$} \\
\hline control & 88 & 0 & 0 \\
p35S::ZmAIL5 & 67 & 1 & 0 \\
p35S::ZmIAA20 & 74 & 0 & 1 \\
p35S::ZmIAA12 & 69 & 1 & 0 \\
p35S::ZmGA20ox2 & 69 & 0 & 1 \\
\hline
\end{tabular}

*Represents the total number of immature zygotic embryos used for three experimental replicates. 20-30 embryos were used per replicate.

Table 4.7: Results of particle bombardment overexpression experiment in sorghum BTx623

\begin{tabular}{lccc}
\hline & $\begin{array}{c}\text { \# immature zygotic } \\
\text { embryos trans- } \\
\text { formed* }\end{array}$ & $\begin{array}{c}\text { \# embryogenic calli } \\
\text { formed }\end{array}$ & \multicolumn{2}{c}{$\begin{array}{c}\text { \# somatic embryos } \\
\text { counted }\end{array}$} \\
\hline control & 60 & 0 & 0 \\
p35S::ZmAIL5 & 54 & 0 & 0 \\
p35S::ZmIAA20 & 58 & 0 & 0 \\
p35S::ZmIAA12 & 63 & 0 & 0 \\
p35S::ZmHb1 & 58 & 0 & 0 \\
\hline
\end{tabular}

*Represents the total number of immature zygotic embryos used for two experimental replicates. 25-35 embryos were used per replicate.

Table 4.8: Results of Agrobacterium-mediated transformation DEX-inducible expression experiment in sorghum Macia

\begin{tabular}{lrcr}
\hline & $\begin{array}{c}\text { \# immature zy- } \\
\text { gotic embryos } \\
\text { transformed* }\end{array}$ & $\begin{array}{c}\text { \# embryogenic calli } \\
\text { formed }\end{array}$ & $\begin{array}{c}\text { \# somatic embryos } \\
\text { counted }\end{array}$ \\
\hline control & 55 & 1 & 1 \\
pOp6::SbWUS;SbBBM & 69 & 1 & 1 \\
pOp6::SbWUS;SbIAA12 & 66 & 2 & 3 \\
pOp6::SbWUS;SbNAC76 & 61 & 0 & 0 \\
pOp6::SbWUS;SbHSCF1 & 58 & 2 & 2 \\
pOp6::SbWUS;SbAIL5 & 58 & 2 & 1 \\
pOp6::SbWUS;SbGLP2-1 & 63 & 1 & 5 \\
PHP78891 & 70 & 4 & 2 \\
\hline
\end{tabular}

*Represents the total number of immature zygotic embryos used for two experimental replicates. 25-35 embryos were used per replicate. 
Table 4.9: Results of Results of Agrobacterium-mediated transformation DEX-inducible expression experiment in sorghum P898012

\begin{tabular}{lrrr}
\hline & $\begin{array}{c}\text { \# immature zy- } \\
\text { gotic embryos } \\
\text { transformed* }\end{array}$ & $\begin{array}{c}\text { \# embryogenic calli } \\
\text { cassette delivered }\end{array}$ & $\begin{array}{c}\text { \# somatic embryos } \\
\text { counted }\end{array}$ \\
\hline control & 77 & 10 & 14 \\
pOp6::SbWUS;SbBBM & 88 & 8 & 17 \\
pOp6::SbWUS;SbHSCF1 & 74 & 7 & 13 \\
pOp6::SbWUS;SbAIL5 & 78 & 9 & 9 \\
PHP78891 & 73 & 7 & 18 \\
\hline *Represents the total number of immature zygotic embryos used for two experimental replicates. \\
30-45 embryos were used per replicate.
\end{tabular}




\section{Chapter 5}

\section{Conclusion}


Accomplishing genetic engineering in plants can be conceptualized as depending on two constituent processes: the first being successful delivery and genomic integration of exogenous genetic material into plant cells, and the second being the ability to regenerate transformed cells into mature, fertile whole plants. Currently, there are many plant species and individual genotypes for which recovery of transgenic plants cannot be reliably achieved due to constraints on one or both of these processes. The work described in this manuscript sought to examine, using differential gene expression analysis performed on RNA-seq data, the plant genes involved in one of the commonly utilized modes of plant regeneration (somatic embryogenesis), and the most widely used technique for transgene delivery into plant tissue (Agrobacterium-mediated transformation). Together, these studies provide resources which could benefit future research into both the basic mechanistic understanding of these processes and on practical applications that serve to improve the capacity of transformation for trait improvement in plants by addressing factors that inhibit transgene delivery or tissue regeneration.

For many important crop species including maize and sorghum, the genotypes that are routinely used for transformation are often not agronomically productive lines. Thus, in order to deploy a new genetically engineered trait for use in a commercial plant variety, the trait must first be incorporated into a "transformable" line and then transferred to elite breeding lines by a trait introgression process consisting of an initial cross followed by several successive backcrossing steps. This process can take up to several years to accomplish and can involve production and testing of thousands of offspring, 
leading to substantial costs in facilities, space, and labor as well as limiting the responsiveness of biotechnology to shifting environmental challenges and market demands. Developing new techniques that contribute to alleviating the genotype-specificity constraints on crop transformation could have a profound impact on the speed at which plant developers are able to implement trait improvements. Rapid progress over the last few years on the application of genome editing tools such as CRISPR/Cas9 in plants further underscores the need for breakthroughs in plant transformation as there is now the potential for using biotechnology to develop plant varieties carrying novel traits which, at least in the United States, will be regulated no differently than varieties produced through conventional breeding methods.

In the study on differential gene expression during early somatic embryogenesis induction in Sorghum bicolor, both broad-scale trends and more granular patterns of differential regulation in individual genes were highlighted. Modules of genes involved in processes such as the auxin-activated signaling pathway, regulation of transcription and translation, and glutathione metabolism were activated in explant tissues treated with 2,4-D relative to the control condition. Genes involved in processes including photosynthesis, and the biosynthesis of fatty acids, starch, and secondary metabolites were generally repressed in the treatment condition. Multiple transcription factors families, including AP2-like, LOB, NAC, and bHLH had several members which were markedly upregulated between conditions. No members of the latter two families have been previously reported as influencing somatic embryogenesis. Several members of the WRKY 
transcription factor family were downregulated in the treatment condition at the earliest time-point comparisons, suggesting this group may be repressed by $2,4-D$ induction. A few epigenetic modulator genes putatively involved in covalent alteration of DNA or of histones were upregulated in the treatment condition. Taken together, this module of genes points toward a concerted program of transcriptional repression that may be important for enabling the cellular reprogramming necessary for somatic embryogenesis. Examination of hormone-related genes which were differentially regulated showed, predictably, that auxin-responsive genes were highly upregulated upon 2,4-D treatment, though it also revealed activation of genes involved in negative regulation of cytokinin signaling and genes involved in the transport and signaling response of abscisic acid. This study adds to a growing body of literature describing transcriptional dynamics surrounding the somatic embryogenesis process and points out several individual genes that merit further investigation into their potential role in the molecular mechanism underlying the developmental process.

Exploration of the host gene expression changes in response to infection with either avirulent or virulent strains of Agrobacterium tumefaciens uncovered the differential expression in several genes that have not previously been implicated in the Agrobacterium-plant interaction. Groups of genes like heat-shock proteins, endochitinases, and peroxidases were induced by both Agrobacterium treatments, whereas genes associated with normal growth and development were repressed, denoting a characteristic host response to perception of pathogen-associated molecular patterns. Transcription 
factors upregulated upon Agrobacterium treatment included the GRAS family transcription factor SCL14, the lateral organ boundaries transcription factor $\angle B D 40$, and MYBR2R3 transcription factor MYB15 as well as many other members of the AP2/ERF, C2H2, NAC, MYB-like, and WRKY families. Contrary to the initial hypothesis that Agrobacterium may suppress the host defense response through the in planta activity of its transferred virulence factors, the virulent strain appeared to more highly induce defense responserelated genes than the avirulent strain. There were relatively few genes which displayed a differential response between virulent and avirulent Agrobacterium treatments. However, a group of four type A ARR genes (involved in negative regulation of cytokinin signaling) were consistently upregulated in the virulent condition at the 6,12 , and 24-hour time-points. These trends, along with the expression trends of other genes involved in cytokinin biosynthesis, perception, and catabolism, provide evidence for the coordinated suppression of cytokinin signaling unique to treatment with the virulent strain. Again, this study emphasizes many individual differentially expressed genes, which can serve as potential leads for further study into the mechanistic underpinning of the plant defense response to Agrobacterium or successful T-DNA delivery.

The purpose of the sorghum transformation work that was reported here was to attempt to validate potential roles for candidate genes identified from the previous sorghum RNA-seq study in regulating somatic embryogenesis in sorghum. Despite multiple experimental approaches in multiple genetic backgrounds, no such functional validation was achieved. There are several reasons this might be the case, which may include bad 
luck in the selection of candidate genes, the insufficiency of methods for producing a detectable phenotype due to functional redundancy in the case of RNAi, the use of orthologous genes from maize for overexpression experiments rather than the sorghum candidate genes themselves, the selection of an inappropriate genotype for overexpression testing, or using a leaky inducible expression construct in the case of the final experiment. It is also possible that screening for increased somatic embryo regeneration over the relatively short time period of transient transformation of sorghum explants is not suitable for detecting effects on somatic embryogenesis for most genes, even if the gene in question actually does have regulatory control over the process.

The identification and functional testing of genes putatively involved in somatic embryogenesis has often been reported in Arabidopsis and Nicotiana tabacum where there are established assays for doing so and stable transgenic plants can be generated over a short timeframe. However, discoveries in these model plants are not guaranteed to translate to useful functional information in other plant species, particularly the more distantly related monocot crops. On the other hand, production and testing of stable transgenic events for several candidate genes in cereal crops would require a substantial investment of time, effort, and resources that would be infeasible for most research groups. Thus, in order to ensure a high probability of success in identifying key genes in somatic embryogenesis, either a high-throughput assay for evaluating candidate genes in cereal crops would need to be developed, or other screening experiments (e.g., ChIPseq or yeast two-hybrid using the BBM protein) would need to be performed to narrow 
down the pool of candidate genes suggested by an RNA-seq differential expression experiment. Additionally, given the general recalcitrance of sorghum varieties to transformation along with tissue culture difficulties arising from the tendency for some varieties to strongly secrete phenolic compounds, maize may be a better system for characterizing monocot developmental regulators as it has already been shown to be widely responsive to $B B M / W U S$-induced regeneration in reports from researchers at Corteva.

Though the work described in this manuscript examined the gene expression responses during 2,4-D-induced somatic embryogenesis and during Agrobacterium-mediated transformation and there is some apparent overlap between the genes that are affected by these two distinct processes, the differentially expressed gene sets are not comparable between the two experiments in any useful way. This is the case due to factors such as the use of different plant species, the treatment of different target explants in different tissue culture contexts, and the different time frames over which samples were taken. However, being able to directly compare the effects of Agrobacterium transformation, somatic embryogenesis, or a combination of both on gene expression could be highly informative for developing techniques to help improve the transformation process. An experiment examining both processes, in which explants of an economically important crop species are either inoculated with Agrobacterium before being plated on callus induction medium or are transferred directly to callus induction medium (with appropriate negative control treatments for both), after which samples of each are collected for whole transcriptome sequencing, would shed light on ways that these processes work either synergistically or antagonistically. For instance, is cytokinin 
signaling repressed in both treatments? Or does Agrobacterium infection induce the expression of a suite of defense genes that have an inhibitory effect on regeneration? The value of such an experiment would be enhanced if another experimental factor were added by performing the same treatments on explants of two different crop genotypes that are known to display differential amenability to transformation.

In addition to identifying genes that might be able to improve the capacity for transformation when their expression is altered by transgenic methods, data such as that produced in the studies reported here could lead to advances in biotechnological methods by other means. For example, these data may inform choices with regard to hormone amendments used in tissue culture media. Alternatively, the data may suggest the application of different classes of chemical compounds (e.g., antioxidants, enzyme inhibitors) that either ameliorate a negative effect of Agrobacterium infection on regeneration or that enhance Agrobacterium infection by mitigating the release of anti-microbial factors by explants in tissue culture. Increasing the amount of genomic, epigenomic, transcriptomic, and metabolomic data on components of the transformation process in increasingly diverse plant species will be a key factor in developing genotype-independent genetic engineering methods for all major crop plants. 


\section{VITA}

Christopher Jacob Willig was born June $4^{\text {th }}, 1990$ in Kansas City, Missouri to his parents, Jennifer Lou Willig (née Bovard) and Clarence Edward Willig Jr. He is the oldest of four boys. Christopher graduated from Lathrop High School in Lathrop, Missouri in 2009. He then went on to attend Missouri State University in Springfield, Missouri for three years before transferring to University of Missouri-Kansas City where he graduated the following year with a B.S. in Biology. He worked for a year as a naturalist at Martha Lafite Thompson Nature Sanctuary in Liberty, Missouri before being accepted into a Ph.D. program in the Division of Plant Sciences at the University of Missouri-Columbia beginning in the fall semester of 2014, with funding from the Life Sciences Fellowship program. Christopher joined the laboratory of Dr. Zhanyuan Zhang in the MU Plant Transformation Core Facility and undertook a research program studying crop transformation techniques and the genetic mechanisms underpinning them. During the last two years of his graduate education, Christopher was supported by a predoctoral fellowship through the USDA-NIFA-AFRI Educational and Workforce Development program. After being awarded his Ph.D., Christopher will begin a postdoctoral research position in the laboratory of Dr. Steven Strauss at Oregon State University where he will work on developing transformation and gene editing techniques in hop. 